\title{
Spin Transport in Organic Semiconductors
}

\author{
Shu-Jen Wang \\ Selwyn College \\ University of Cambridge
}

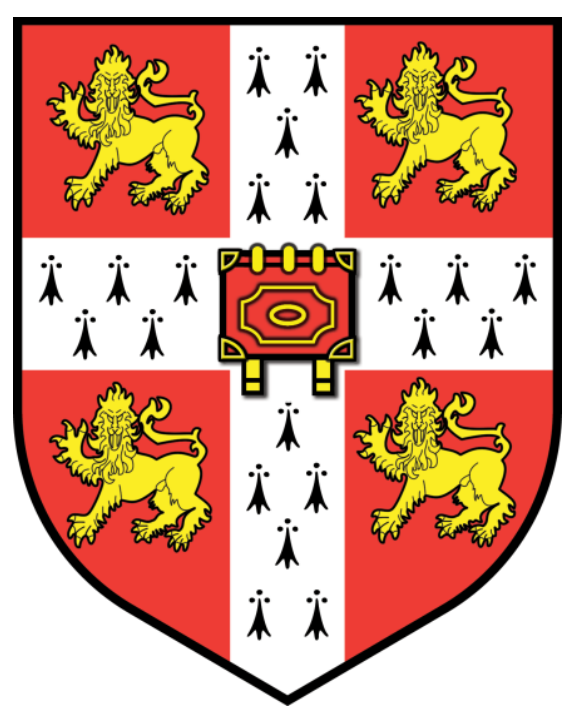

A dissertation submitted for the degree of Doctor of Philosophy

August 2018 
To my parents for their endless support... 


\section{Abstract}

The unique physical properties and low temperature solution processability of organic semiconductors have enabled many applications such as light emitting diodes, flexible logic and solar cells, they are unexploited in their potential for use in solid state devices for spintronics and spin-based information processing. Organic semiconductors composed of mainly light elements appeal to the field of spintronics due to their long spin lifetime originating from their weak spin-orbit coupling. The significant progress in improving carrier mobility of organic semiconductors in the past decade may lead to organic spin transport materials with both long spin diffusion length and spin lifetime which is important for spintronics applications. This dissertation explores the spin transport in organic semiconductors using a variety of experimental techniques from all electrical spin injection and detection to ferromagnetic resonance spin pumping and ISHE spin detection.

Non-local spin valves and novel all electrical spin transport device architectures based on high mobility conjugated polymers were studied systematically. The intrinsic roadblocks for electrical spin injection-based measurements were identified as the current spreading effect (electrical cross-talk between the injector and detector electrodes) and the hopping conduction in organic semiconductors which makes all electrical nonlocal spin injection and detection measurements extremely challenging if not impossible for organic semiconductors. In addition, spin current transmission in the out of plane direction of organic semiconductors was studied by tri-layer spin pumping technique where the spin transport properties of organic semiconductors are correlated with their molecular structure and charge transport properties.

Spin pumping, a charge-free spin injection method together with ISHE spin detection successfully overcome the impedance mismatch problem and the intrinsic roadblocks imposed by electrical spin injection-based techniques and enabled lateral spin current transport in organic semiconductors to be detected electrically. The lateral spin diffusion length of up to a micrometre was observed in doped conjugated polymers in agreement with theoretical calculations based on exchange mediated spin diffusion model and parameters obtained from first principle. Moreover, this non-local spin transport device structure provides a platform for studying spin transport in a wide range of organic semiconductors where the spin current propagates along the high mobility direction and could potentially be used as building blocks for high performance flexible spintronics devices. 


\section{Acknowledgement}

First, I would like to thank my supervisor, Professor Henning Sirringhaus for providing me the opportunity to join his research team and guiding me throughout my time in his group. His insights toward the projects and science in general have benefited me significantly. I would also like to thank my Selwyn College tutor, Dr. Gavin Jarvis for providing me with his full support towards both my academic and non-academic life.

Moreover, I would like to especially thank Dr. Deepak Venkateshvaran who has mentored me and worked very closely with me on various projects. His wisdom and passion towards scientific research have made an important impact to my research career. I am very fortunate to have the chance to work closely with such an intelligent and humble academic in Cambridge. I would also like to Dr. Riccardo Di Pietro who has trained me on various equipments and involved me in his own project.

Furthermore, I would like to thank Dr. Radoslav Chakalov and Roger Beadle for maintaining the lab and assisting me with various lab tasks. I want to thank my collegues in OE-FET for their help and useful discussion (in particular, Dr. Keehoon Kang, Dr. Murat Cubukcu, Dr. Stuart Higgins, Dr. Guillaume Schweicher, Dr. Ekaterina Selezneva, Angela Wittmann, Chen Chen, Sam Schott, Olga Zadvorna, Piotr Skalski).

In addition, I also want to thank Dr. Jöerg Wunderlich and Thomas Wagner at Hitachi Cambridge Laboratory for useful discussion and assistance with various measurements. I would also like to thank all the collaborators who have contributed to the projects outside Cambridge within the ERC synergy network. Professor Iain McCulloch, Dr. Mark Little and Cameron Jellet at Imperial College for providing us with high-quality organic semiconductors and Professor Jairo Sinova, Dr. Erik McNellis, Dr. Reza Mahani, Dr. Sergei Egorov, Sebastian Müller and Uday Chopra at Johannes Gutenberg Universität, Mainz for first principle theoretical calculations and modelling.

Finally, I would like to acknowledge Universities New Zealand for a Scholarship (20142017). I would also like to thank European Research Council Synergy Grant for providing me with financial support for conference and meeting attendance. Last but not least, I want to thank my family for raising me and providing me with their full support. 


\section{Preface}

This dissertation contains the work carried out at the University of Cambridge between October 2014 and August 2018 as a $\mathrm{PhD}$ student. Except where specific reference is made to the involvement of others, the work presented here is my own and contains nothing which is the result of collaboration. No part of this dissertation has been submitted for the award of a degree at this or any other university. The length of this work is within the limit of 60,000 words.

Shu-Jen Wang

Selwyn College, Cambridge

August 2018 


\section{Table of contents}

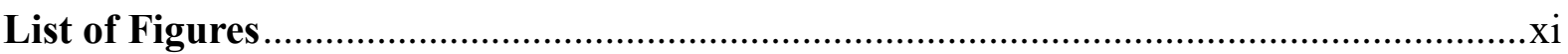

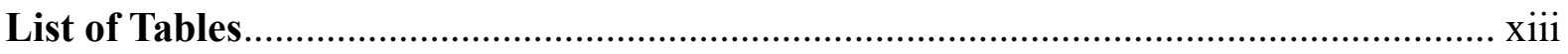

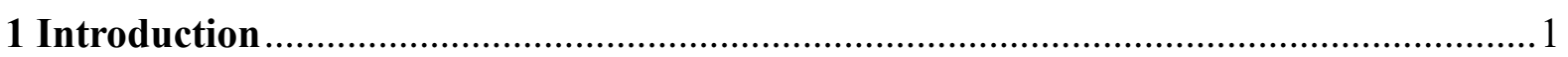

1.1 Emergence and applications of organic semiconductors .......................................... 1

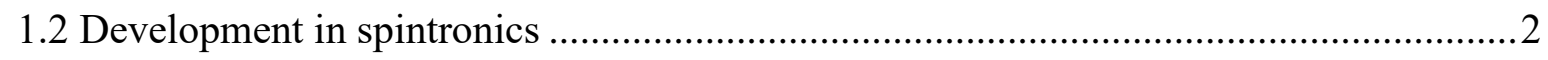

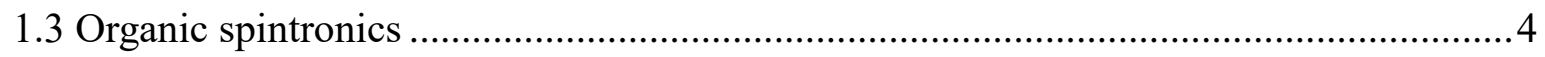

2 Theory

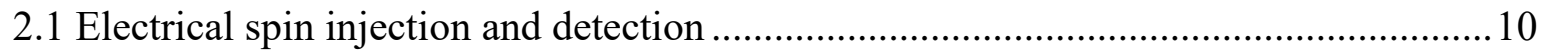

2.1.1 Vertical spin valves and electrical spin injection conditions ............................... 10

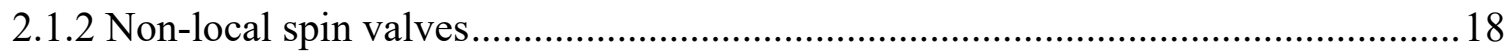

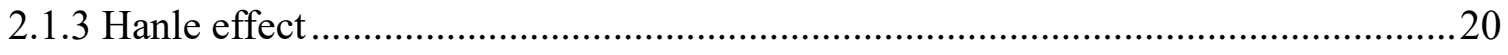

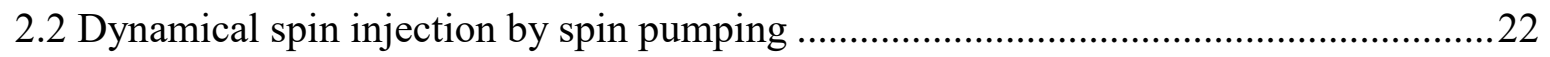

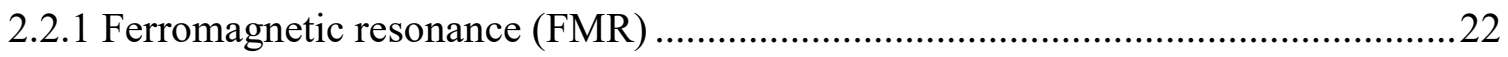

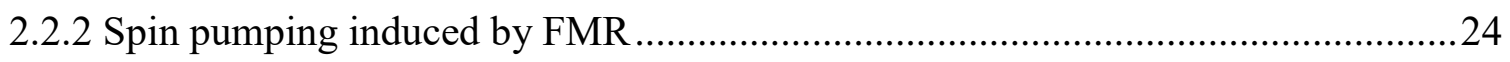

2.2.3 Spin Hall effect (SHE) and Inverse spin Hall effect (ISHE) .................................26

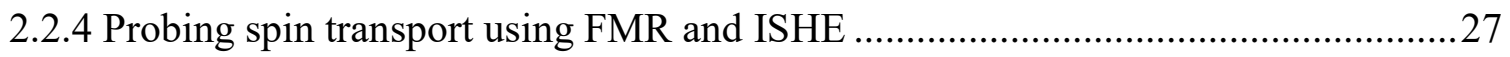

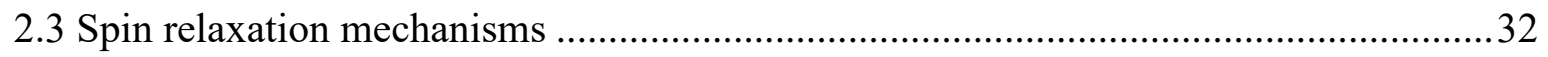

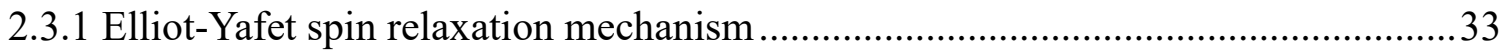

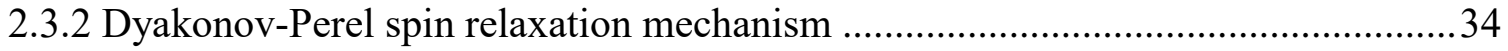

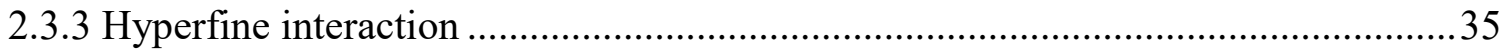

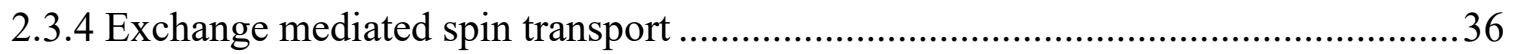

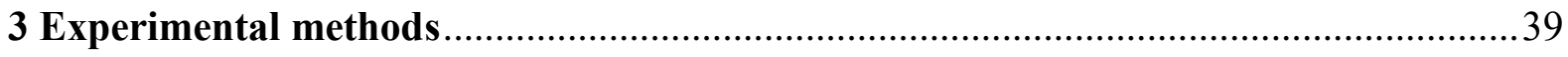

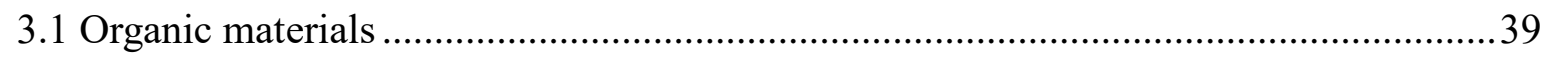

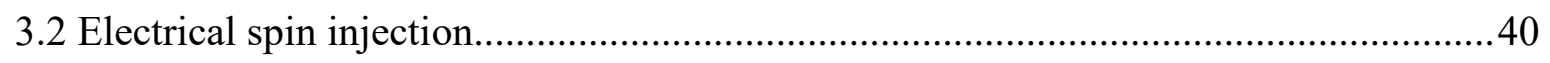

3.2.1 Vertical spin valves fabrication .................................................................. 40

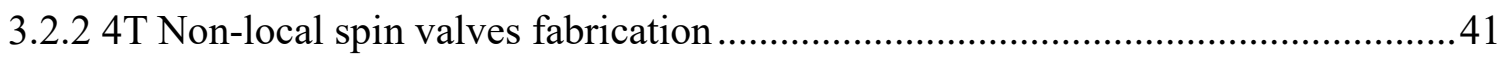


3.2.3 Out of plane spin injection and in plane spin detection spin valves fabrication 41

3.2.4 Electrical spin injection and inverse spin hall effect detection devices fabrication. 42

3.2.5 Electrical spin injection measurements 42

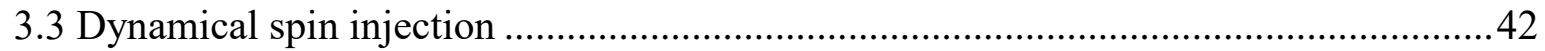

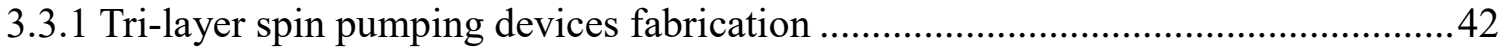

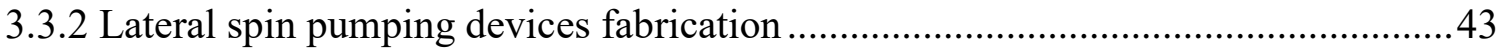

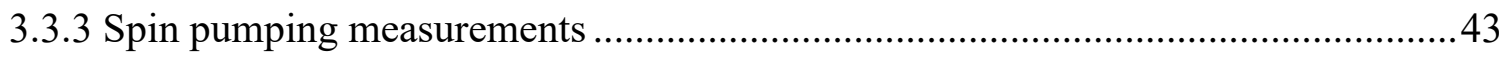

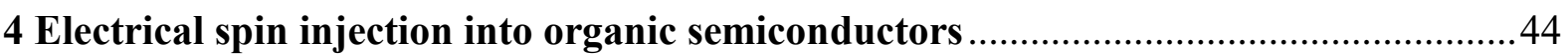

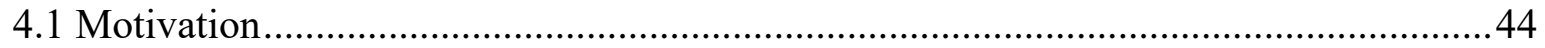

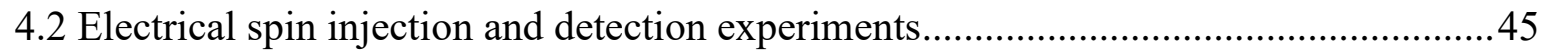

4.2.1 Charge injection properties across ferromagnet/organic semiconductor interfaces 45

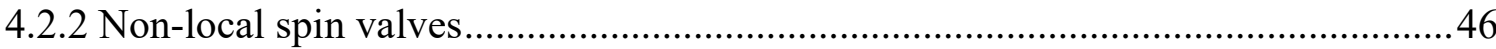

4.2.3 Out of plane spin injection and in plane spin detection spin valves .......................51

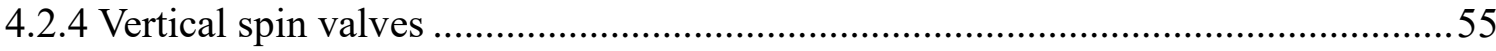

4.2.5 Electrical spin injection and ISHE detection devices ......................................59

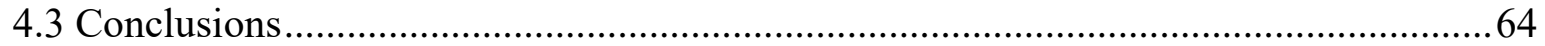

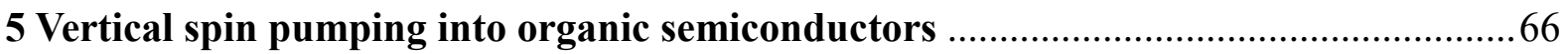

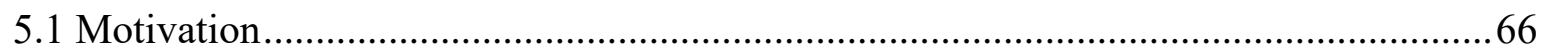

5.2 Pure spin current transport in organic semiconductors using tri-layer spin pumping

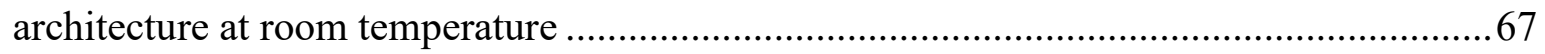

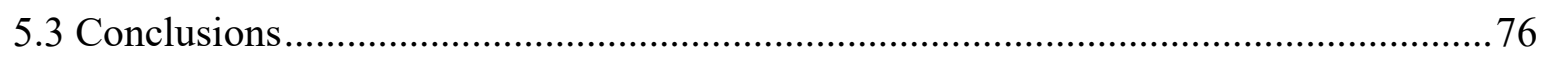

6 Lateral spin transport in organic semiconductors probed by spin pumping and ISHE .78

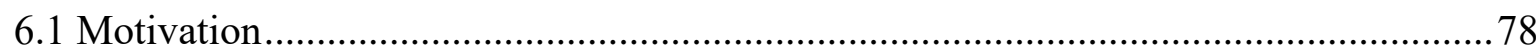

6.2 Observation of lateral spin transport in organic semiconductors at room temperature .79

6.3 Out of plane angular, temperature and frequency dependence measurements ..............84

6.4 Electrical characterisation and additional control experiments .................................. 88

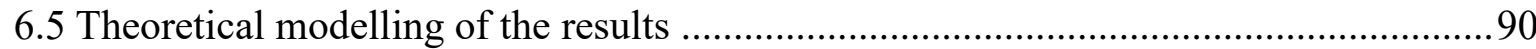




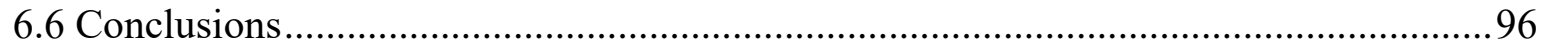

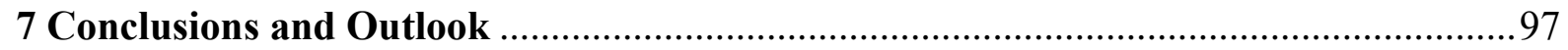

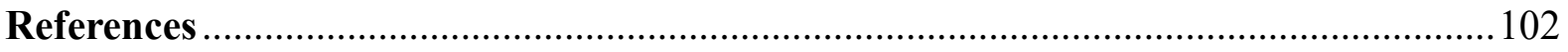

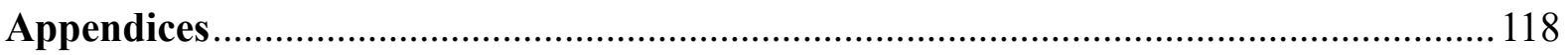

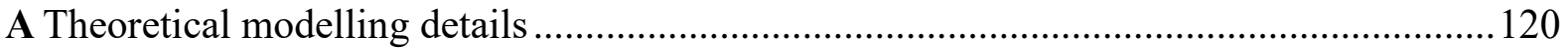

B Spin precession simulations (Hanle Effect) and measured spin lifetimes in organic semiconductors

C Lithography processes details

D Publications and Presentations... 


\section{List of Figures}

Figure 1.1 Roadmap for spin diffusion length, $l_{\mathrm{s}}$ versus spin lifetime, $\tau_{\mathrm{s}}$ for various organic and inorganic materials. .5

Figure 1.2 GMR observed in two terminal Alq3 based spin valve....................................... 7

Figure 2.1 Schematic illustration of the giant magnetoresistance effect. ............................... 12

Figure 2.2 Working principle of GMR based read head................................................. 13

Figure 2.3 Efficient spin injection conditions for metallic ferromagnet and semiconductor

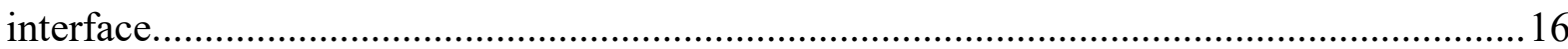

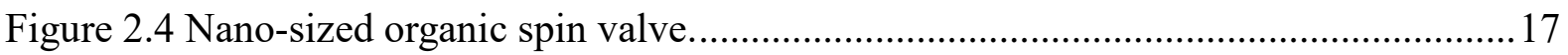

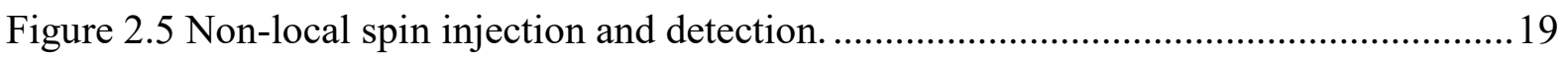

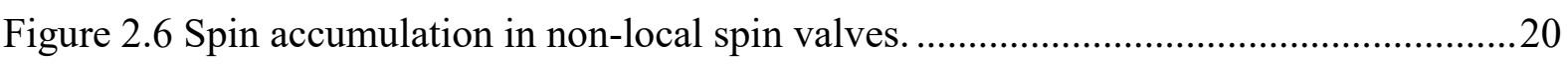

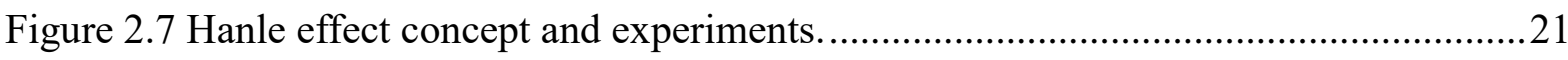

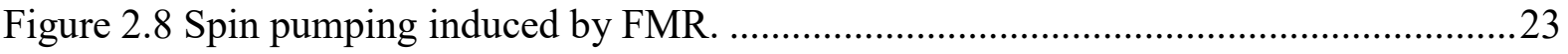

Figure 2.9 Schematic illustration of magnetic field and magnetisation angle definition. .......23

Figure 2.10 Schematic illustrations of spin Hall effect and inverse spin Hall effect..............27

Figure 2.11 Lateral spin pumping and ISHE detection in p-Si.......................................... 30

Figure 2.12 Lateral spin pumping control experiment and spin diffusion length extraction...31

Figure 2.13 Schematic demonstration of Elliot-Yafet and Dyakonov-Perel spin relaxation

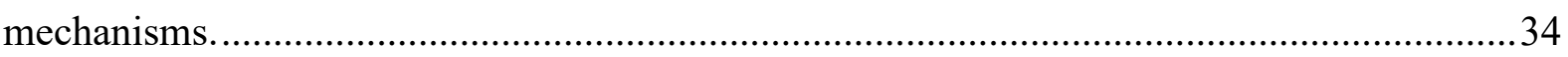

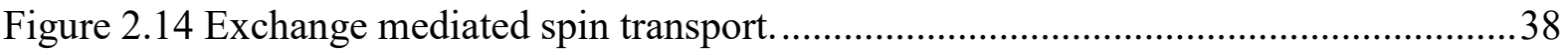

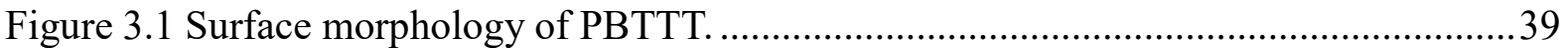

Figure 3.2 Molecular structures of main organic semiconductors studied. ............................40

Figure 4.1 Electrical spin injection interface properties. .................................................... 46

Figure 4.2 Non-local spin valves based on organic semiconductors. .................................. 49

Figure 4.3 Organic non-local spin valves measurements. ................................................50

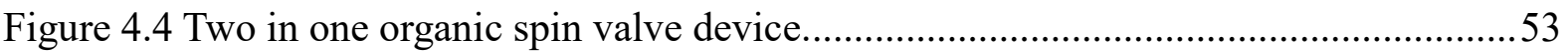

Figure 4.5 Out of plane spin injection and in plane spin detection measurement. .................54

Figure 4.6 Large area $(200 \times 200 \mu \mathrm{m})$ vertical organic spin valve device............................56

Figure 4.7 Magnetoresistance of vertical organic spin valve...............................................58

Figure 4.8 Electrical spin injection into organic semiconductor and ISHE detection. ............60

Figure 4.9 Electrical spin injection and ISHE detection measurement-current spreading effect. 
Figure 4.10 Ultralow noise Hall effect in rubrene signal crystal/PFPE.

Figure 5.1 Tri-layer spin pumping measurements.

Figure 5.2 Pure spin current transport in organic conjugated polymers at room temperature. 71

Figure 5.3 Spin current transmission in conjugated organic polymers at room temperature. .73

Figure 5.4 Tri-layer spin pumping control experiments.

Figure 5.5 Out of plane angular dependence of the ISHE signal in the tri-layer spin pumping

devices at room temperature. . .75

Figure 5.6 Molecular-metal Rashba spin to charge converter. ............................................. 77

Figure 6.1 Lateral spin pumping device architecture and measurement scheme. ..................80

Figure 6.2 Observation of long range spin transport in F4TCNQ doped PBTTT. ..................82

Figure 6.3 Carrier density dependence of spin current transport......................................... 84

Figure 6.4 Out of plane angular dependence of the ISHE signal in the lateral spin pumping device. .86

Figure 6.5 Temperature dependence of the ISHE signal. .87

Figure 6.6 Frequency dependence of FMR of Py. .88

Figure 6.7 Electrical characterisation of the lateral spin pumping device and additional control experiments. .90

Figure 6.8 Theoretical modelling of spin transport in an exchange mediated regime. .95

Figure 7.1 Lateral spin pumping and ISHE detection for different materials at room temperature.

Figure 7.2 Lateral spin pumping and ISHE detection for Rubrene single crystal device at room temperature.

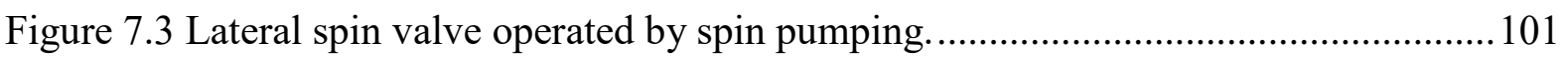

Figure A.1 Polaron (hole) density along $\pi$-stacking direction. .......................................... 123

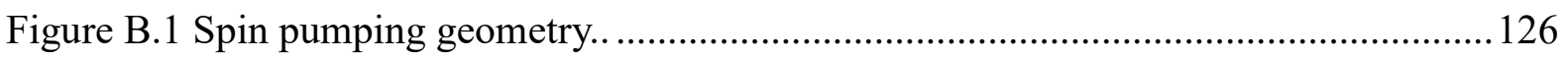

Figure B.2 Schematic illustration of injected spin polarization........................................ 127

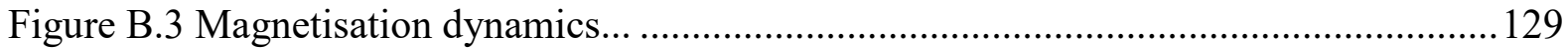

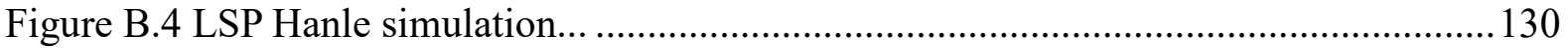

Figure B.5 Ion-exchanged PBTTT ESR and UV-vis spectrum........................................ 131 


\section{List of Tables}

Table 1.1 Spin diffusion lengths of organic semiconductors extracted using two terminal vertical spin valves along with the electrode materials used.

Table 2.1 Spin diffusion length and spin hall angles of commonly used materials in spintronics.

Table 2.2 Comparison between the spin diffusion lengths of various materials estimated by lateral spin pumping and conventional four terminal non-local spin valve techniques............31

Table A. $1 \chi 2$ calculated from DFT for PBTTT and P3HT. 122 


\section{Chapter 1}

\section{Introduction}

\subsection{Emergence and applications of organic semiconductors}

Organic semiconductors have enabled a technology transformation in the electronics industry with practical applications in display, light emitting diode and solar cell technology. These materials are a class of materials which consists of mainly $\mathrm{C}$ and $\mathrm{H}$ atoms. Their semiconducting nature results from $\pi$-conjugation, which arises due to alternating single and double bonds along the backbone of $\mathrm{C}$ atoms where each $\mathrm{C}$ atom has three $s p^{2}$-hybridised orbitals used to create $\sigma$-bonds with neighbouring $\mathrm{C}$ and $\mathrm{H}$ atoms. This bonding arrangement along the backbone results in overlapped $\pi$-orbitals to form delocalised electron clouds where charge transport takes place. Organic semiconductors have two energy bands, highest occupied molecular orbital (HOMO) and lowest occupied molecular orbital (LUMO). The HOMO and LUMO levels are the bonding and antibonding $\pi$-orbitals of the delocalised electron clouds which can be compared to the valence and conduction bands in conventional inorganic semiconductors [1].

One important advantage of organic semiconductors over crystalline inorganic semiconductors is the ability to be processed at relatively low temperature. The reason for this property is that organic semiconductors are held together via weak Van der Waals interactions while inorganic semiconductors are held together by strong covalent bonds. The compatibility of OSCs with low cost solution processing route enables large area ink-jet printing of OSCs into active layers for flexible optoelectronics applications [2].

There are generally two classes of OSCs, molecular semiconductor and conjugated polymer semiconductor. Molecular semiconductors are made of small molecules such as, Tris(8hydroxyquinolinato)aluminium $\left(\mathrm{Alq}_{3}\right)$ whereas conjugated polymer semiconductors are made of polymers such as, poly(3-hexylthiophene-2,5-diyl) (P3HT) and poly[2,5bis(3tetradecylthiophen-2-yl)thieno[3,2-b]thiophene] (PBTTT). The weak electronic interactions 
between concomitant molecules limit the mobility of OSCs due to weak Van der Waals interactions that hold the OSCs together. This also limits the bandwidth of charge transport in OSCs to the order of $0.1 \mathrm{eV}$ [3]. In contrast, conventional inorganic semiconductors typically have a bandwidth on the order of 1-10 eV due to strong electronic interactions between atoms which arise from strong covalent bonds between atoms. As such, charge transport in organic semiconductors are often referred to as narrow bandwidth transport [3].

Even though the mobility of organic semiconductor is far lower than high quality Silicon, OSCs have made a significant impact to the electronics industry. In particular, organic light emitting diode (OLED) has emerged as the next generation display technology and is expected to dominate in the mobile phone and electronic display market. The rapid emergence of OLED is made possible due to OSCs provide a low cost, flexible, ease of processing and environmentally friendly option compared to the conventional inorganic liquid crystal.

\subsection{Development in spintronics}

The field of spintronics aims at utilising the spin degree of freedom in addition to the conventional charge current based information processing technology [4]. The first spintronics effect that created a paradigm shift in the read head technology is the discovery of giant magnetoresistance (GMR) which is based on two ferromagnetic electrodes separated by a metallic layer. One ferromagnetic electrode is pinned magnetically while the other ferromagnetic electrode is free to change magnetic orientation where one electrode injects spin polarised current and the other acts as a detector for spin polarised current. The detection mechanism is based on the fact that the spin-up and spin-down densities of states at the Fermi Level depend on the magnetisation orientation of the ferromagnetic electrode (please refer to Chapter 2.1.1 for more in-depth discussion about the GMR theory). Therefore, the resistance of the stack depends on the relative orientation of the ferromagnetic electrodes which is used to read the memory stored as magnetic bits in hard disk read head technology.

This disruptive technology breakthrough has revolutionised the modern computer capacity. For this, Grunberg and Fert received Nobel prize in Physics in 2007 for this discovery [5], [6].

Based on the GMR success, innovative ideas and device concepts emerge rapidly to improve this technology. Magneto-resistive random access memory (MRAM) based on tunnelling magnetoresistance (TMR) opened up a market for non-volatile magnetic memory. TMR is 
more efficient than GMR since it overcomes the impedance mismatch problem at ferromagnet and metal interface by using a tunnelling barrier. Much research has been made to maximise the TMR ratio, it was found for $\mathrm{MgO}$ barrier with certain crystalline orientation combined with ferromagnetic electrode with certain crystalline orientation such as iron the TMR ratio can reach hundreds of percent due to symmetry spin filtering results from synergy between the electronic structures of $\mathrm{MgO}$ and tunnelling barrier [7], [8]. MRAM technology can be combined with spin transfer torque (STT) to manipulate the orientation of the ferromagnetic element by spin polarised current where a torque acts on the magnetic layer via application of spin angular momentum [9]. STT-MRAM technology offers the potentials for an energy efficient and fast operating non-volatile memory for next generation memory devices. This technology has shown commercialisation prospects where test products have been delivered to customers [10].

The experimental observation of spin hall effect (SHE) has opened up many exciting opportunities for spintronics research. This effect enables the generation of pure spin current from charge current in non-magnetic material through spin orbit coupling. It is important to note that pure spin current is a flow of spin angular momentum without a net charge current as opposed to spin polarised current [11]. In contrast, the inverse spin hall effect (ISHE) converts pure spin current into charge current via spin orbit coupling [11]. These effects not only expand the materials systems relevant for spintronics either as the spin current generator or detector but also enable new device architectures and measurement configurations for exploration of novel physical phenomenon such as spin hall effect transistor and spin seebeck effect (SSE) [12], [13]. Further improvements to STT-MRAM technology can be realised by incorporating SHE where instead of using a spin polarised current to manipulate the magnetic element, a pure spin current generated from SHE can be used to manipulate the orientation of the magnetic element [Spin orbit torque (SOT)] [14], [15], [16]. In this device configuration, current is only flowing through the material with strong spin orbit coupling and hence the tunnelling barrier is not exposed to large current density during the memory writing process. This significantly improves the durability of the SOT-MRAM device compares to STT-MRAM device.

It is to be noted that the progress in the field of spintronics has been rigorous, many novel breakthroughs have been achieved such as Skyrmions, inverse Rashba-Edelstein effect and spin Nernst effect [17], [18], [19]. These discoveries are not only of scientific interest but also offer a variety of potential applications. The ever miniaturisation of integrated circuits will soon reach the Moore's law limit, the computing performance and capacity can only improve by 
finding an alternative avenue. The development of artificial intelligence, internet of things and other ICT technologies requires further advances in the computer performance. In addition, the excessive heat generated from the current computation technology as well as the energy consumption will unavoidably limit the ICT technology development. Evolvement from charge current based spintronics (GMR) to dissipationless pure spin current spintronics could potentially provide a solution to this problem where pure spin current is used to fuel efficient and low power magnetic logic circuits.

\subsection{Organic spintronics}

Despite the fact that organic semiconductors have enabled many applications such as organic light emitting diodes, transistors and solar cells, they are unexploited in their potential for use in solid state devices for spintronics and spin-based information processing [20], [21]. OSCs have attracted much interest in the domain of spintronics due to their exceptionally long spin lifetimes arising from weak spin orbit coupling due to their composition of primarily light elements [22]. This is in contrast with inorganic semiconductors such as Silicon or GaAs where the atomic numbers of the constituent elements are larger, giving rise to stronger spin-orbit coupling. Spin dependent scattering induced by strong spin orbit coupling is known to limit the distance over which spin information can be propagated [11]. As such, the scientific community sees an opportunity to design an OSC material that exhibit both long spin lifetime and spin diffusion length that could potentially be used for quantum information processing [21], [23], [24]. The ideal spin transport material for quantum computation needs have a spin lifetime that is long enough for the information stored as spins to be detected and manipulated. In addition, the spin diffusion length of the spin transport materials also needs to be long enough such that external stimuli can be incorporated into the devices such as spin transistors (channel gap dimensions limited by lithography resolution). Moreover, the number of ways to engineer the chemical structures of OSCs is almost infinite. This provides much potential for tailoring the spin related properties of OSCs for specific applications. 


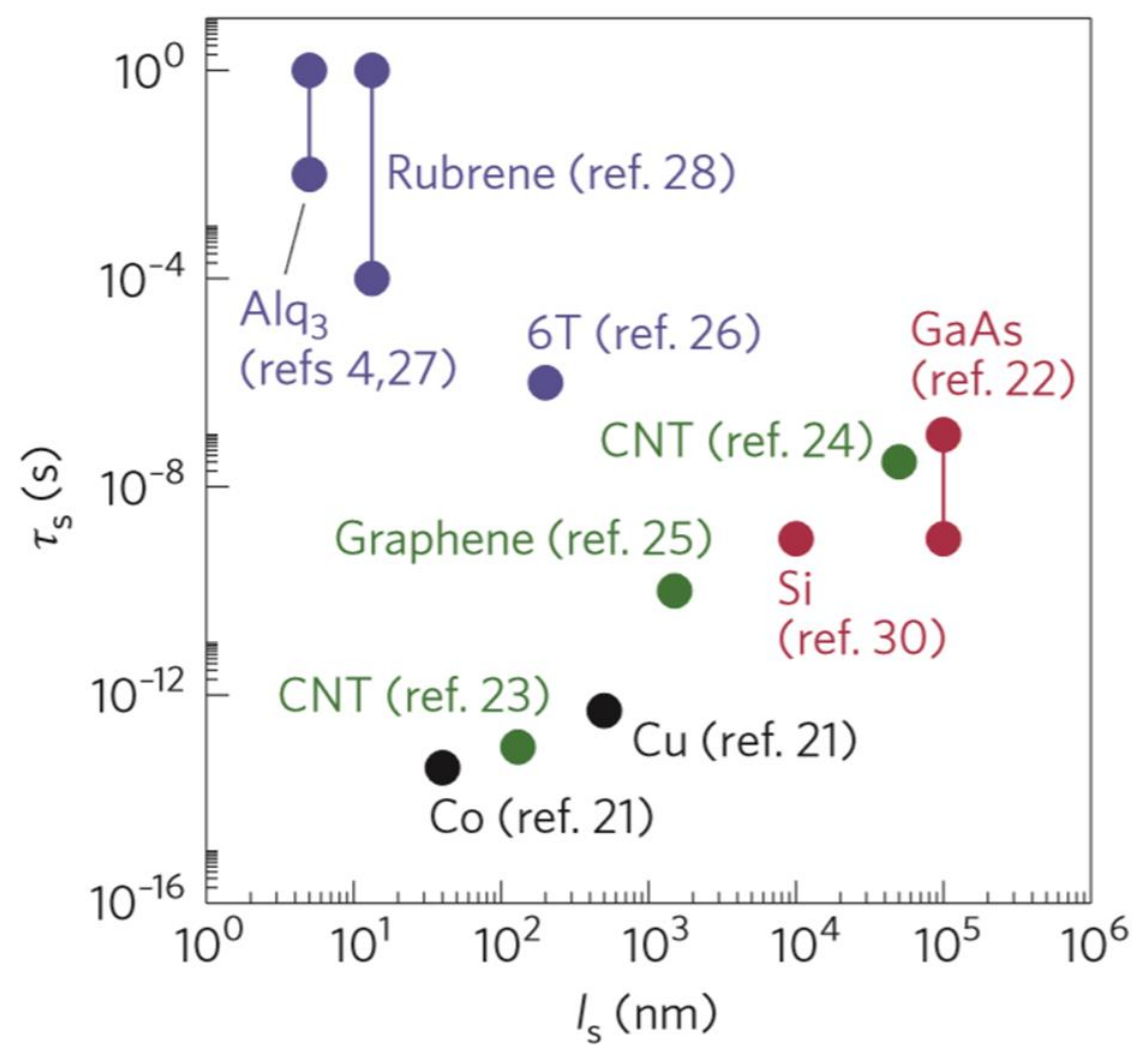

Figure 1.1 Roadmap for spin diffusion length, $l_{\mathrm{s}}$ versus spin lifetime, $\tau_{\mathrm{s}}$ for various organic and inorganic materials. This figure is modified from [23].

The physical properties of interfaces between hybrid organic and inorganic materials have gained much attention in the recent past due to the emergence of unconventional physical properties at the interfaces between organic and inorganic materials. The emergence of magnetism in C60 and copper interfaces due to charge transfer between the organic material and metal is an example of unexpected physical property observed at hybrid organic-inorganic interface [25]. Similarly, organic materials strongly modify the spin injection and magnetic properties of organic-inorganic ferromagnet interfaces via interfacial interactions [26]. The molecular spin properties can potentially be exploited for molecular based memory applications [21]. Thus, understanding the hybrid organic-inorganic interface is important both scientifically and technologically. In addition, the intrinsic organic magnetoresistance (OMAR) in OSCs is another interesting research area within organic spintronics where large magnetoresistance over $10 \%$ was observed in OLED structure at room temperature within small applied magnetic field (typically around tens of mT) [27], [28]. Although the mechanisms that caused OMAR effect is under debate, the field is gaining consensus that OMAR originates from the hyperfine field of $\mathrm{H}$ nuclei [29], [30]. Apart from the scientific interest, OMAR can potentially be useful for innovative applications such as OLED based touch display controlled 
by a magnetic pen [31].

Organic spintronics research in the past decade primarily focuses on two terminal vertical spin valves where organic semiconductors sandwiched between two ferromagnetic electrodes. In this architecture, spin polarised current is injected from one electrode into the organic semiconductor and detected by the other electrode as GMR. Xiong et al was the first to use such measurement to study the spin diffusion length in $\mathrm{Alq}_{3}$ by fitting an exponential function to the thickness dependence of GMR signal measured [32]. This approach was used to study spin transport in many other OSCs including polymers such as D-DOO-PPV [30], H-DOOPPV [30], C60 [33], TIPS-Pentacene [34], RR-P3HT [35], and P(NDI2OD-T2) [36] yielding spin diffusion lengths on the order of $50 \mathrm{~nm}$. The results are summarised in Table 1.1.

Table 1.1 Spin diffusion lengths of organic semiconductors extracted using two terminal vertical spin valves along with the electrode materials used.

\begin{tabular}{|c|c|c|}
\hline $\begin{array}{l}\text { Organic } \\
\text { Semiconductor }\end{array}$ & Electrodes & Spin diffusion length $\left(\lambda_{\mathrm{s}}\right)$ \\
\hline $\begin{array}{l}\text { Alq }_{3} \\
\text { D-DOO-PPV } \\
\text { H-DOO-PPV } \\
\text { TIPS-Pentacene } \\
\text { P(NDI2OD-T2) } \\
\text { C60 }_{\text {RR-P3HT }} \\
\text { RR-P3HT }\end{array}$ & $\begin{array}{l}\text { LSMO, Co } \\
\text { LSMO, Co } \\
\text { LSMO, Co } \\
\mathrm{CoPt}, \mathrm{AlO}_{x} / \mathrm{Co} \\
\mathrm{LSMO}, \mathrm{Co} \\
\mathrm{Fe}_{3} \mathrm{O}_{4} / \mathrm{AlO}_{x}, \mathrm{Co} \\
\mathrm{LSMO} \mathrm{Co} \\
\mathrm{FeCo}, \mathrm{NiFe}\end{array}$ & $\begin{array}{l}45 \mathrm{~nm} \text { at } 11 \mathrm{~K} \mathrm{[32]} \\
45 \mathrm{~nm} \text { at } 10 \mathrm{~K} \mathrm{[30]} \\
16 \mathrm{~nm} \text { at } 10 \mathrm{~K}[30] \\
24 \mathrm{~nm} \text { at } 300 \mathrm{~K}[34] \\
64 \mathrm{~nm} \text { at } 4.2 \mathrm{~K} \mathrm{[36]} \\
150 \mathrm{~nm} \text { at } 300 \mathrm{~K} \mathrm{[33]} \\
63 \mathrm{~nm} \text { at } 50 \mathrm{~K}[37] \\
62 \mathrm{~nm} \text { at } 300 \mathrm{~K}[38]\end{array}$ \\
\hline
\end{tabular}



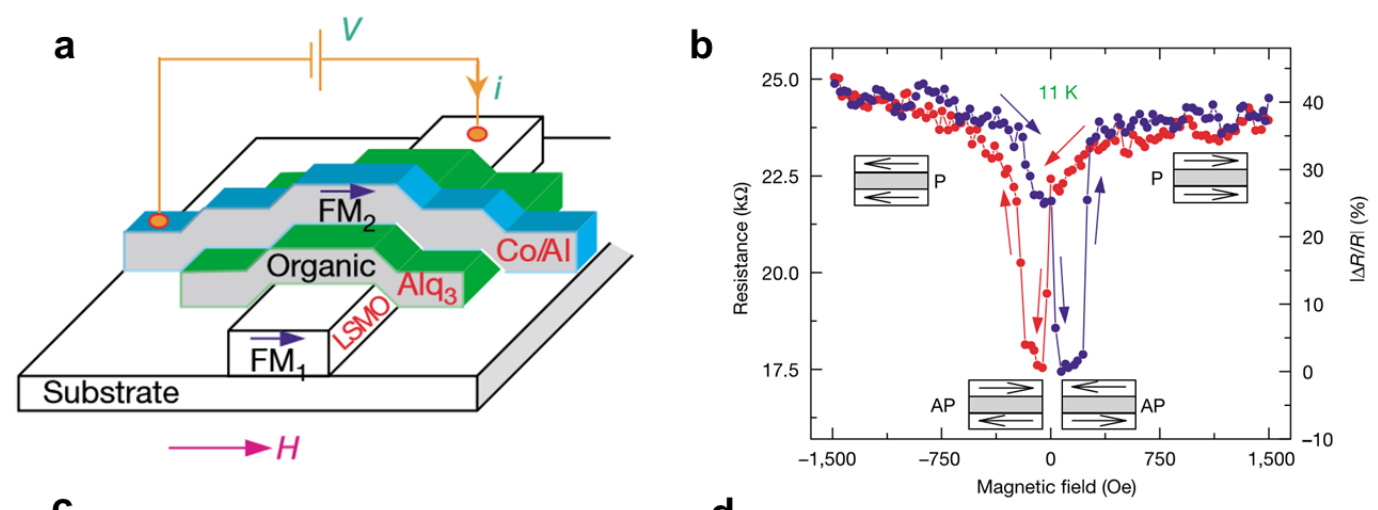

C

d
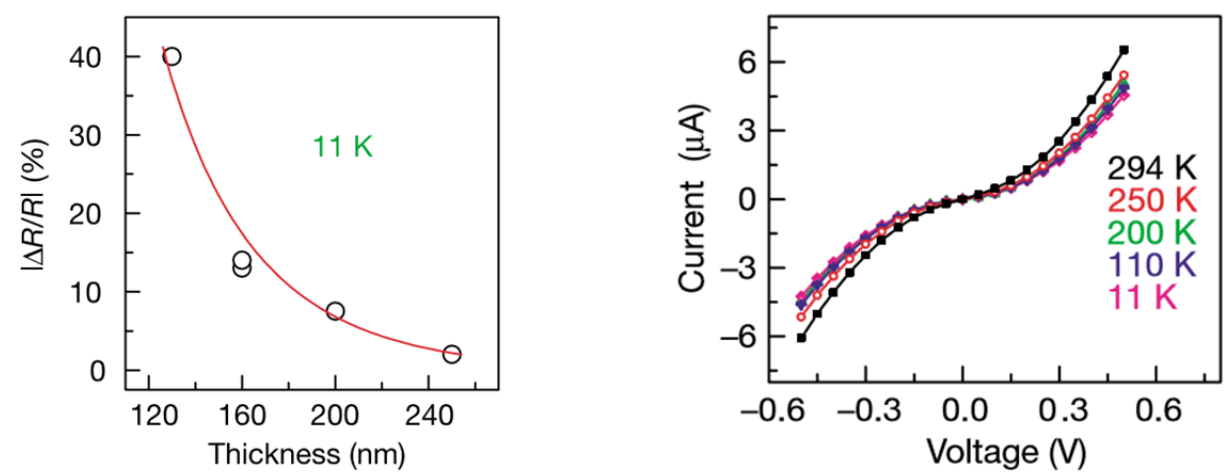

Figure 1.2 GMR observed in two terminal Alq3 based spin valve. a, Schematic illustration of the vertical spin valve structure where Alq3 is sandwiched between LSMO and $\mathrm{Co} / \mathrm{Al}$ ferromagnetic electrodes. b, Magnetoresistance observed at $11 \mathrm{~K}$ when the electrodes magnetisation alignment switches from parallel to antiparallel. c, Thickness dependence of GMR observed at $11 \mathrm{~K}$. A $45 \mathrm{~nm}$ spin diffusion length can be extracted by fitting an exponential function to the plot. d, Temperature dependence of $I-V$ characteristics of the spin valve device. This figure is modified from [32].

Although such a measurement of spin diffusion lengths in organic spin valves has become routine, the majority of reported devices used Lanthanum Strontium Manganate (LSMO) as one of their ferromagnets in the tri-layer stacks. LSMO is known to have a strong bi-axial anisotropy and it is now clear that what was routinely reported and interpreted as a magnetoresistance signal in organic spin valves may have arisen from the phenomenon of Tunnelling Anisotropic Magnetoresistance (TAMR) within the LSMO contact [39]. In addition, organic semiconductors are also vulnerable to metal atom inter-diffusion during the top metal layer deposition due to the weak structural properties of OSCs. It was recently demonstrated that a reduction in the junction area of an organic spin valve causes the measured magnetoresistance to vanish, signalling the role pinholes play in large area organic spin valves [40]. The presence of such pinholes within the organic spacer layer makes it difficult to distinguish whether spin transport in these devices is diffusive or ballistic. 
There have been many attempts to validate and confirm spin injection and transport in OSCs. Inelastic electron tunnelling spectroscopy (IETS) in addition to the trilayer spin valve measurement can rule out the presence of metallic pin holes by probing the relevant molecular vibration spectra [41], [42], [43]. Conduction mediated by charge carriers hopping is thermally activated which means at lower temperature resistivity increases as carriers freeze out. A temperature dependent electrical characterisation of the trilayer spin valve device is carried out in conjunction with the magneto-transport measurements in which devices that showed the correct temperature dependence of device resistance can be used to rule out metallic shorts, pin holes and other defects between the ferromagnetic electrodes [44]. However, depending on the dimensions of the pin holes and other defects, this argument may not be valid [40], [41]. Other sophisticated experimental techniques such as optically detected magnetic resonance (ODMR), two photon photoemission, spin OLED and muon spin rotation confirm successful spin injection and spin transport in the OSCs [30], [45], [46] [47]. However, ambiguities such as the aforementioned in the reported measurements from tri-layer spin valves calls into question the adequacy of many of the existing results, and necessitates alternative artefact-free approaches to probing spin transport in organic semiconductors.

An unambiguous solid-state device based approach to measure spin diffusion in inorganic semiconductors is to use nonlocal lateral architectures where spin diffusion is decoupled from spin drift within the same device [48], [49]. Pure diffusive spin transport is then probed by measuring the spin accumulation above a ferromagnetic detector electrode in relation to that at the ferromagnetic injector. Such nonlocal spin valve measurements have successfully quantified spin diffusion lengths in inorganic materials such as graphene, Ge, GaAs and various metals [50], [51], [48], [52], [49], [53]. In conjugated organic semiconductors however, a nonlocal spin valve signal has never been successfully measured despite multiple diligent attempts. This is primarily because the noise fluctuations on the measured nonlocal voltage are induced by the fundamental nature of stochastic hopping transport in these disordered materials, and outsizes the spin accumulation on the detector electrode rendering such a measurement extremely challenging if not impossible [54], [55].

Spin pumping has become a popular technique to study spin transport and spin to charge conversion in a wide variety of materials such as metals, graphene and inorganic semiconductors [56], [57], [58], [59]. It involves exciting a ferromagnetic material into ferromagnetic resonance in which pure spin current is injected into the adjacent material in 
proximity via exchange interaction at the interface. The major advantage of this spin injection technique is that it overcomes the impedance mismatch problem that significantly limits spin injection in the case of electrical spin injection [60]. Ando et al were the first to demonstrate spin to charge conversion in conducting polymer, poly(3,4-ethylenedioxythiophene) poly(styrenesulfonate) (PEDOT:PSS) where yttrium iron garnet (YIG) was excited into FMR and the pure spin current injected into the organic material was converted into a charge current by inverse spin hall effect [61]. This technique was extended further to study the spin transport in organic semiconductors where organic material is sandwiched between a ferromagnetic metal layer and a heavy metal layer [62]. In this configuration, the ferromagnetic metal is driven into FMR and pure spin current is injected into the organic material. The pure spin current that arrives at the heavy metal layer is converted into a charge current via the ISHE of the heavy metal. This measurement architecture has much less restriction on the conductivity of the organic material than the spin to charge conversion measurement because the ISHE voltage is measured across the highly conductive heavy metal electrode. Thus, the spin physics of a wide range of OSCs may be studied by this method. 


\section{Chapter 2}

\section{Theory}

\subsection{Electrical spin injection and detection}

\subsubsection{Vertical spin valves and electrical spin injection conditions}

A vertical spin valve is GMR based device which consists of a layer of spin transport material sandwiched between two ferromagnetic electrodes. The two ferromagnetic electrodes have different coercive fields such that they can align parallel or antiparallel by applying external magnetic field. In the case of normal GMR, the resistance of the spin valve device is the lowest when the magnetisation orientations of the ferromagnetic electrodes are aligned parallel and vice versa. Spin polarised electrons are injected from one ferromagnetic electrode and detected via the adjacent ferromagnetic electrode. Depending on the magnetisation orientation of the electrode, the difference between spin-up and spin-down electron density of states changes as illustrated in Fig 2.1. Therefore, when the magnetisation alignment of the electrodes is parallel, spin-down electrons can flow from ferromagnetic electrode 1 to ferromagnetic electrode 2 easily (low resistance) since there are spin-down electron density of states at Fermi level (Fig 2.1a). In contrast, when the magnetisation alignment of the electrodes is antiparallel, it is difficult for spin-down electrons to flow from ferromagnetic electrode 1 to ferromagnetic electrode 2 (high resistance) since there is no spin-down electron density of state at Fermi level (Fig 2.1b). The magnetoresistance response (MR) in spin valve devices is given by $\left(R_{\mathrm{ap}}-R_{\mathrm{p}}\right) / R_{\mathrm{p}}$ where $R_{\mathrm{p}}\left(R_{\mathrm{ap}}\right)$ is the device resistance for parallel (antiparallel) magnetisation alignment of the ferromagnetic electrodes.

Observation of GMR effect enabled the modern hard disk read head that lead to significant advances in memory technology. GMR based read head consists of a spin valve element where one magnetic electrode is pinned while the other electrode (soft) is free to change its magnetisation direction (Fig 2.2). The magnetic field that emanate from the magnetic domain of magnetic storage media switches the magnetisation of the soft ferromagnetic electrode. By 
monitoring the resistance of the GMR element, the magnetic bits stored using magnetic domains can be sensed accurately.

An organic spin valve uses organic conductor/semiconductor material sandwiched between two ferromagnetic electrodes. Spin diffusion length $\left(\lambda_{\mathrm{s}}\right)$ of the organic material can be extracted by fitting an exponential decay function, $\mathrm{e}^{-d / \lambda}$ to the thickness dependence $(d)$ of magnetoresistance measurement. Spin diffusion length can be correlated to mobility $(\mu)$ and spin lifetime $(\tau)$ of the carriers using Einstein relationship,

$$
\lambda s=\sqrt{\mu k_{B} T \tau / e}
$$

where $k_{\mathrm{B}}, T$ and $e$ are the Boltzmann constant, temperature and the elementary charge, respectively. Organic semiconductors typically have very long spin lifetimes due to their weak spin-orbit coupling and hyperfine interaction. However, due to their low carrier mobility compared to inorganic semiconductors, their spin diffusion lengths are limited to around 50 $\mathrm{nm}$. If their carrier mobility can be improved, it is possible to have an organic semiconductor with both long spin lifetime and spin diffusion length based on Eq. (2.1). The magnetoresistance of vertical organic spin valves can be modelled by a modified Julliere's formula that the decay of spin polarisation of the spin polarised carriers in the organic semiconductor is considered as given in Eq. (2.2).

$$
M R=\frac{2 P_{1} P_{2} e^{-d / \lambda s}}{1+P_{1} P_{2} e^{-d / \lambda s}}
$$

where $P_{1}$ and $P_{2}$ are the spin polarisation of top and bottom ferromagnetic electrodes, respectively. 


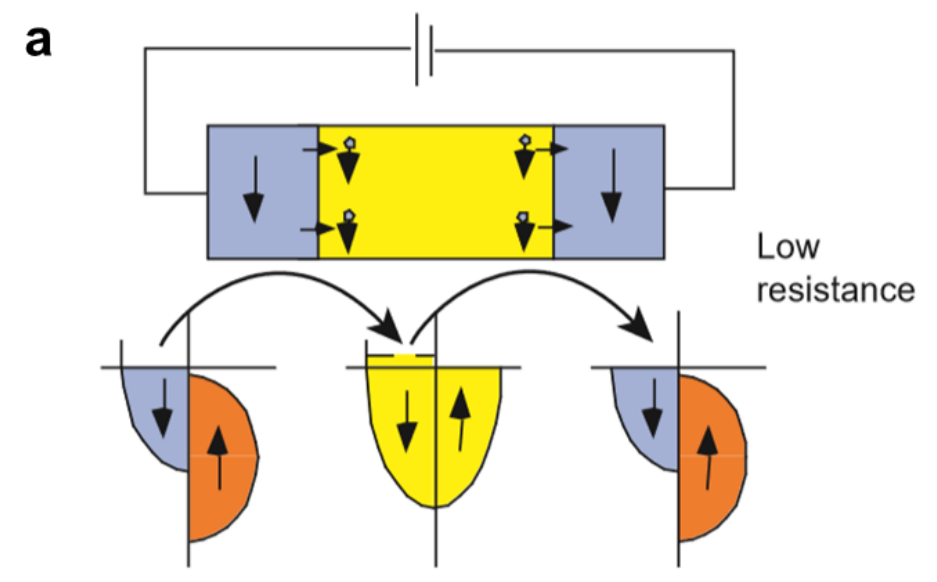

b

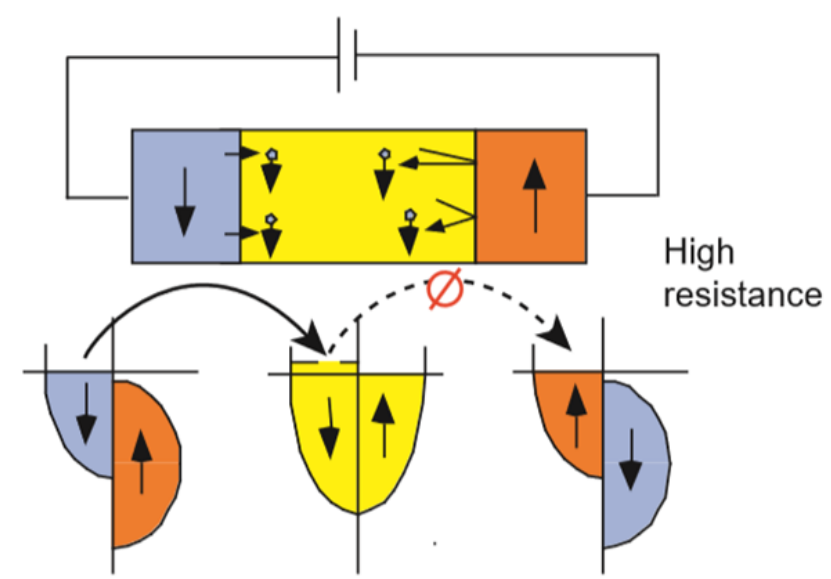

Figure 2.1 Schematic illustration of the giant magnetoresistance effect. A spin valve structure consists of a normal metal layer (yellow) spaced between two ferromagnetic electrodes. Spin polarised carriers are injected from one ferromagnetic electrode and detected by the adjacent ferromagnetic electrode. a, When the ferromagnetic electrodes are parallel, the resistance of the stack is low. $\mathbf{b}$, When the ferromagnetic electrodes are antiparallel, the resistance of the stack is high. The reason for this spin valve effect is due to the difference in density of states between spin-up and spin-down electrons. The figure is modified from [63]. 


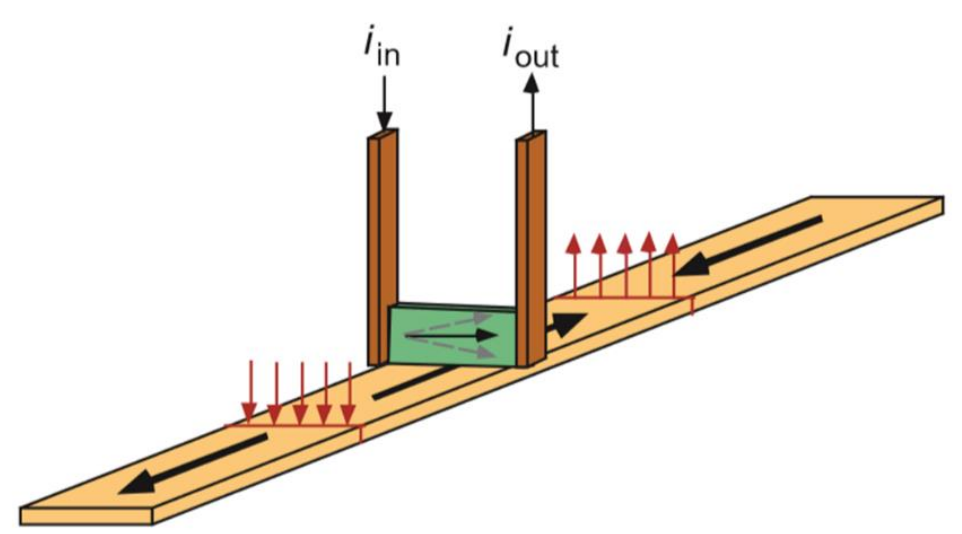

Figure 2.2 Working principle of GMR based read head. The GMR read head (green) is made of a spin valve device that one ferromagnetic electrode is pinned permanently while the other ferromagnetic electrode (soft) is free to switch its magnetisation. The magnetisation of the soft magnetic layer is switched by the magnetic field that originates from the magnetic recording media. The magnetisation direction of the magnetic domain is used to store data information as binary data either " 0 -magnetised towards right" or "1-magnetised towards left". The resistance of the element can therefore be used to sense the magnetisation direction of the magnetic domain in the recording media. This figure is modified from [63].

Conductivity mismatch problem impedes successful spin injection into semiconductor material using metallic ferromagnet injector. The spin polarisation of current density $(\alpha)$ is given by Eq. (2.3),

$$
\alpha=\frac{j_{\uparrow}-j_{\downarrow}}{j_{\uparrow}+j_{\downarrow}}
$$

where $j_{\uparrow}$ and $j_{\downarrow}$ denote current densities for spin-up and spin-down electrons, respectively.

Schmidt et al have approximated theoretically that $\alpha$ is proportional to the ratio of the semiconductor conductivity $\left(\sigma_{\mathrm{SC}}\right)$ and the ferromagnetic metal conductivity $\left(\sigma_{\mathrm{FM}}\right)[64]$ :

$$
\alpha \propto \frac{\sigma_{\mathrm{SC}}}{\sigma_{\mathrm{FM}}}
$$

It is therefore difficult to maintain spin polarised current in the semiconductor as the conductivity difference between semiconductor and metal is often orders of magnitude different.

Fig 2.3a shows the spin polarisation of current as a function of distance from the interface between a ferromagnet and non-magnet. The spin polarisation of current at the interface, $(\alpha)_{\mathrm{I}}$ is given by Eq. (2.5) where the full derivation can be found in ref [65]. 


$$
(\alpha)_{\mathrm{I}}=\left(\frac{J_{\uparrow}-J_{\downarrow}}{J}\right)_{\mathrm{I}}=\frac{\beta r_{\mathrm{F}}+\gamma r_{\mathrm{b}}{ }^{*}}{r_{\mathrm{F}}+r_{\mathrm{N}}+r_{\mathrm{b}}{ }^{*}}
$$

where $\beta$ and $\gamma$ denote bulk spin asymmetry coefficient of the ferromagnetic material and spin asymmetry coefficient of the interface resistance, respectively. $r_{\mathrm{F}}$ and $r_{\mathrm{N}}$ are product of resistivity and spin diffusion length for ferromagnet and non-magnet, respectively. $r_{\mathrm{b}}{ }^{*}$ is the interface resistance area product. For curve 1, the interface is between $\mathrm{Co} / \mathrm{Cu}$ metallic system, Eq. (2.5) can be simplified to $(\alpha)_{\mathrm{I}}=\beta /\left(1+r_{\mathrm{N}} / r_{\mathrm{F}}\right)$. Assuming the resistivity of Co and $\mathrm{Cu}$ to be $7.5 \times 10^{-8} \Omega \mathrm{m}$ and $6 \times 10^{-9} \Omega \mathrm{m}[66]$, respectively and the spin diffusion lengths of $\mathrm{Co}$ and $\mathrm{Cu}$ to be $59 \mathrm{~nm}$ [67] and $1 \mu \mathrm{m}$ [68], respectively, $r_{\mathrm{F}}=4.5 \times 10^{-15} \Omega \mathrm{m}^{2}$ and $r_{\mathrm{N}}=6 \times 10^{-15} \Omega \mathrm{m}^{2}$. Due to similar values of $r_{\mathrm{F}}$ and $r_{\mathrm{N}}$, the spin polarisation of current entering copper is only slightly reduced $\left((\alpha)_{\mathrm{I}}=0.2\right.$ assuming $\left.\beta=0.46[66]\right)$.

For curve 2, the interface is between $\mathrm{Co} /$ semiconductor heterostructure without a tunnelling barrier. Consider the semiconductor is GaAs with $r_{\mathrm{N}} \sim 4 \times 10^{-9} \Omega \mathrm{m}^{2}$, the value of $r_{\mathrm{N}}$ is larger than $r_{\mathrm{F}}$ by six orders of magnitude. The large value of $r_{\mathrm{N}}$ is primarily caused by the much larger resistivity of GaAs compared to metals. This results in a diminishing value of $(\alpha)_{\mathrm{I}}$ at $\mathrm{Co} / \mathrm{GaAs}$ interface as illustrated in Fig 2.3a. This reflects the conductivity mismatch problem discussed earlier.

From Eq. (2.5), it is possible to achieve high valve of $(\alpha)_{\mathrm{I}}$ even though $r_{\mathrm{N}} \gg r_{\mathrm{F}}$ when a tunnelling barrier is inserted between the ferromagnet and non-magnet that provides sufficiently large interface resistance $\left(r_{\mathrm{b}}{ }^{*}>r_{\mathrm{N}}\right)$. For curve 3 , a tunnelling barrier is inserted between $\mathrm{Co} / \mathrm{GaAs}$ where $r_{\mathrm{b}}{ }^{*}=r_{\mathrm{N}}$, the spin polarisation of current at the interface is increased significantly to 0.25 . By tuning the interface resistance of tunnelling barrier $\left(r_{\mathrm{b}}{ }^{*} \gg r_{\mathrm{N}}\right),(\alpha)_{\mathrm{I}}$ approaches $\gamma$. Thin layer of Aluminium oxide $\left(\mathrm{AlO}_{\mathrm{x}}\right)$ is commonly used as high-quality tunnelling barrier with interface resistance ranging from $10^{-10}-10^{-4} \Omega \mathrm{m}^{2}$. The value of $\gamma$ can reach 0.5 for tunnelling from Co based alloys [69].

Fig $2.3 \mathrm{~b}$ inset shows a $\mathrm{F} / \mathrm{N} / \mathrm{F}$ spin valve structure in lateral geometry where a semiconductor (GaAs) is sandwiched between two ferromagnetic metal electrodes with tunnelling barrier contacts. Following the approach described by Valet and Fert [70], expressions that describes the magnetoresistance of the spin valve can be obtained [Eqs. (2.6) and (2.7)]. 


$$
\Delta R=\frac{2\left(\beta r_{\mathrm{F}}+\gamma r_{\mathrm{b}}{ }^{*}\right)^{2}}{\left(r_{\mathrm{b}}{ }^{*}+r_{\mathrm{F}}\right) \cosh \left(\frac{t_{\mathrm{N}}}{l_{\mathrm{sf}} \mathrm{N}}\right)+\frac{r_{\mathrm{N}}}{2}\left[1+\left(\frac{r_{\mathrm{b}}{ }^{*}}{r_{\mathrm{N}}}\right)^{2}\right] \sinh \left(\frac{t_{\mathrm{N}}}{l_{\mathrm{sf}}}\right)}
$$

where $\Delta R$ is the resistance change between antiparallel and parallel magnetisations alignment of the ferromagnetic metal electrodes. $t_{\mathrm{N}}$ and $l_{\mathrm{sf}} \mathrm{N}^{\mathrm{N}}$ denote the GaAs thickness ie gap between two ferromagnetic electrodes and spin diffusion length of GaAs.

$$
\begin{array}{r}
R^{(\mathrm{P})}=2\left(1-\beta^{2}\right) r_{\mathrm{F}}+r_{\mathrm{N}} \frac{t_{\mathrm{N}}}{l_{\mathrm{Sf}}}+2\left(1-\gamma^{2}\right) r_{\mathrm{b}}{ }^{*} \\
+2 \frac{(\beta-\gamma)^{2} r_{\mathrm{F}} r_{\mathrm{b}}{ }^{*}+r_{\mathrm{N}}\left(\beta^{2} r_{\mathrm{F}}+\gamma^{2} r_{\mathrm{b}}{ }^{*}\right) \tanh \left(\frac{t_{\mathrm{N}}}{2 l_{\mathrm{Sf}} \mathrm{N}}\right)}{\left(r_{\mathrm{F}}+r_{\mathrm{b}}{ }^{*}\right)+r_{\mathrm{N}} \tanh \left(\frac{t_{\mathrm{N}}}{2 l_{\mathrm{sf}} \mathrm{N}}\right)}
\end{array}
$$

where $R^{(\mathrm{P})}$ is the resistance of the spin valve when the magnetisations of the ferromagnetic electrodes align parallel. We now consider the magnetoresistance of a spin valve structure that a tunnelling barrier is inserted between the metallic ferromagnetic electrode and GaAs in which Fig 2.3b shows a simulated magnetoresistance as a function of interface resistance and thickness of GaAs. From Eq. (2.6), sizable magnetoresistance will be observed when the value of $r_{\mathrm{b}}{ }^{*}$ lies between $r_{\mathrm{N}}\left(t_{\mathrm{N}} / l_{\mathrm{Sf}} \mathrm{N}^{\mathrm{N}}\right)$ and $r_{\mathrm{N}}\left(l_{\mathrm{sf}} \mathrm{N}^{\mathrm{N}} / t_{\mathrm{N}}\right)$. For $t_{\mathrm{N}} / l_{\mathrm{sf}}{ }^{\mathrm{N}}=0.01\left(t_{\mathrm{N}}=20 \mathrm{~nm}\right)$, the magnetoresistance is maximised in a relatively wide range centred around $6.4 \times 10^{-9} \Omega^{2}$ (two orders of magnitude spread) as illustrated in Fig 2.3b. The maximum magnetoresistance span reduces to around one order of magnitude for $t_{\mathrm{N}} / l_{\mathrm{Sf}} \mathrm{N}^{\mathrm{N}}=0.1\left(t_{\mathrm{N}}=200 \mathrm{~nm}\right)$. Furthermore, the magnetoresistance is maximised in a very narrow range for $t_{\mathrm{N}} / l_{\mathrm{sf}}{ }^{\mathrm{N}}=1\left(t_{\mathrm{N}}=2 \mu \mathrm{m}\right)$. The value of maximum magnetoresistance reduces with increasing thickness of GaAs due to spin relaxation in GaAs. Thus, it is important to consider both the thickness of semiconductor and interface resistance to maximise the magnetoresistance.

To understand the variation of magnetoresistance as a function of interface resistance, interface resistance can be classified into three regions, (1) $r_{\mathrm{b}}{ }^{*}$ much smaller than $r_{\mathrm{N}}\left(t_{\mathrm{N}} / l_{\mathrm{Sf}}{ }^{\mathrm{N}}\right),(2)$ $r_{\mathrm{b}}{ }^{*}$ between $r_{\mathrm{N}}\left(t_{\mathrm{N}} / l_{\mathrm{sf}}{ }^{\mathrm{N}}\right)$ and $r_{\mathrm{N}}\left(l_{\mathrm{sf}^{\mathrm{N}}}{ }^{\mathrm{N}} / t_{\mathrm{N}}\right)$ and (3) $r_{\mathrm{b}}{ }^{*}$ much larger than $r_{\mathrm{N}}\left(l_{\mathrm{sf}}{ }^{\mathrm{N}} / t_{\mathrm{N}}\right)$. In region $1, r_{\mathrm{b}}{ }^{*}$ is too small to overcome the conductivity mismatch problem. Hence, the magnetoresistance achieved in this region is low. In region $2, r_{\mathrm{b}}{ }^{*}$ is sufficiently large to overcome the conductivity mismatch problem while $r_{\mathrm{b}}{ }^{*}$ is not too large to cause an overall increase in device resistance. Therefore, magnetoresistance is maximised in this region. When the value of $r_{\mathrm{b}}{ }^{*}$ increases beyond this region (region 3), the $\Delta R$ saturates at $4 \gamma^{2} r_{\mathrm{N}}\left(l_{\mathrm{sf}}^{\mathrm{N}} / t_{\mathrm{N}}\right)$ [Eq. (2.6)], however, $R^{(\mathrm{P})}$ continues to increase with $r_{\mathrm{b}}{ }^{*}$. Consequently, magnetoresistance drops off in this region. 
a

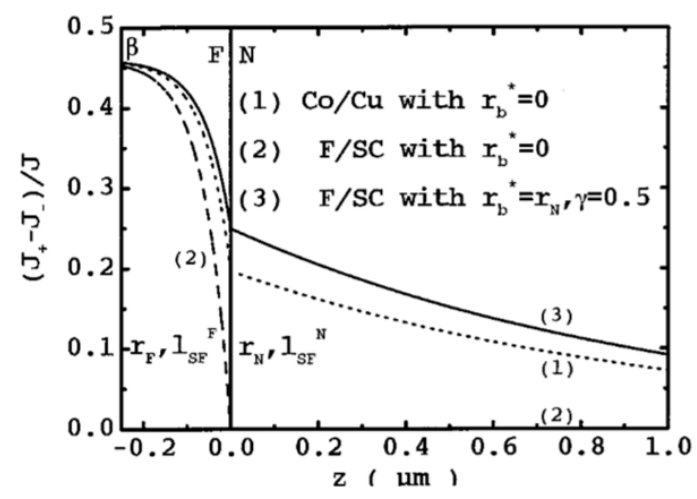

b

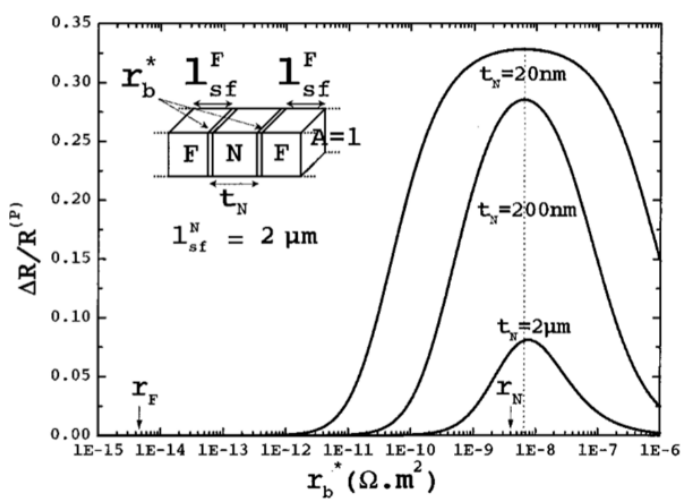

Figure 2.3 Efficient spin injection conditions for metallic ferromagnet and semiconductor interface. a, Spin polarisation of current, $\mathrm{J}_{+}-\mathrm{J}_{-} / \mathrm{J}$ as a function of distance, $\mathrm{z}$ from the ferromagnet/semiconductor $(\mathrm{F} / \mathrm{N})$ interface. Curve 1 is for $\mathrm{Co} / \mathrm{Cu}$ metallic system without interface resistance. Curve 2 is for metallic ferromagnet and semiconductor system without interface resistance. Curve 3 is for metallic ferromagnet and semiconductor system with interface resistance (tunnel barrier). b, Magnetoresistance versus interface resistance, $r_{b}{ }^{*}$ for a spin valve structure as shown in the inset where $\mathrm{F}$ is $\operatorname{Co}\left(r_{\mathrm{F}}=4.5 \times 10^{-15} \Omega \mathrm{m}^{2}\right)$ and $\mathrm{N}$ is semiconductor $\left(r_{\mathrm{N}}=4 \times 10^{-9} \Omega \mathrm{m}^{2}\right)$. The thickness of the semiconductor is varied from $20 \mathrm{~nm}$ to $2 \mu \mathrm{m}$ assuming a spin diffusion length $\left(l_{\mathrm{sf}}^{\mathrm{N}}\right)$ of $2 \mu \mathrm{m}$. This figure is modified from [65].

As discussed earlier, high quality tunnelling barrier can be used to solve the conductivity mismatch problem effectively. In addition, the use of a Schottky barrier could also provide the interface resistance required to overcome the conductivity mismatch problem [48] [71]. The pioneering observation of magnetoresistance in organic spin valve did not use a tunnelling barrier. This is surprising as the conductivity of organic semiconductors is typically far lower than inorganic semiconductors which makes the conductivity mismatch problem more severe for organic semiconductors. However, the charge injection in organic semiconductors is often mediated by variable range hopping which tunnelling transport is involved [72]. Therefore, a natural tunnelling barrier is formed between the metallic ferromagnet and organic semiconductor interface that overcomes the inherent conductivity mismatch problem. Thus, unlike inorganic semiconductors, it is possible to spin inject into organic semiconductors without the need of an artificial tunnelling barrier. This theory has been confirmed by experimental study of nano-sized organic spin valves. The observation of large tunnelling magnetoresistance (over $200 \%$ ) in precision fabricated nano-sized organic spin valve with ultra-thin organic spacer $\left(2 \mathrm{~nm} \mathrm{Alq}_{3}\right)$ verified that organic semiconductors can act as effective tunnelling barriers (Fig 2.4b) [73]. This theory supports experimental observation of magnetoresistance in organic spin valves without tunnelling barrier incorporated between 
metallic ferromagnet and organic semiconductor [32], [74], [75]. Organic materials are vulnerable to the formation of pin holes and defects when subjected to metal evaporations due to their relatively soft physical property. This means the evaporation conditions and junction dimension need to be carefully optimised. Furthermore, they cannot withstand standard lithography patterning since they can be damaged by the solvent during lift-off or uv/e-beam exposure during patterning. Therefore, it is very challenging to microfabricate organic spin valve devices. The nano-sized organic spin valve employed a novel fabrication route to overcome the difficultly associated with microfabrication of organic spin valves. The fabrication starts with $\mathrm{LSMO} / \mathrm{Alq}_{3}$ bilayer covered with insulating resist. The nano-sized junction is then indented by conductive-tip AFM which can precisely define the dimensions of the tunnel junction. The nanohole created by this process is then filled with Co which completes the $\mathrm{LSMO} / \mathrm{Alq}_{3} / \mathrm{Co}$ nanosized organic spin valve.

a

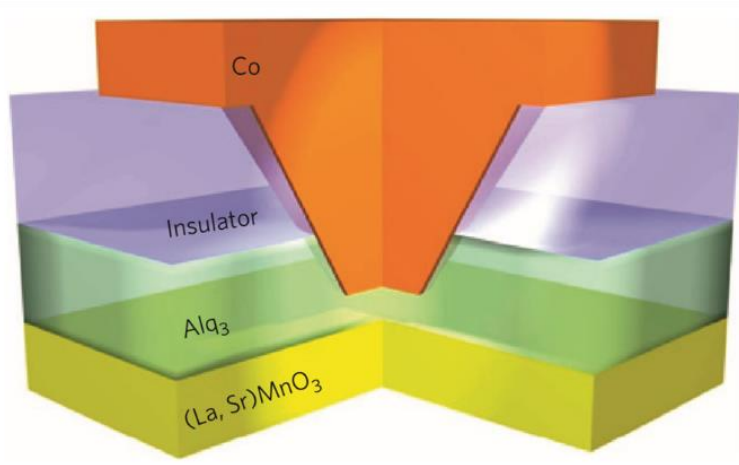

b

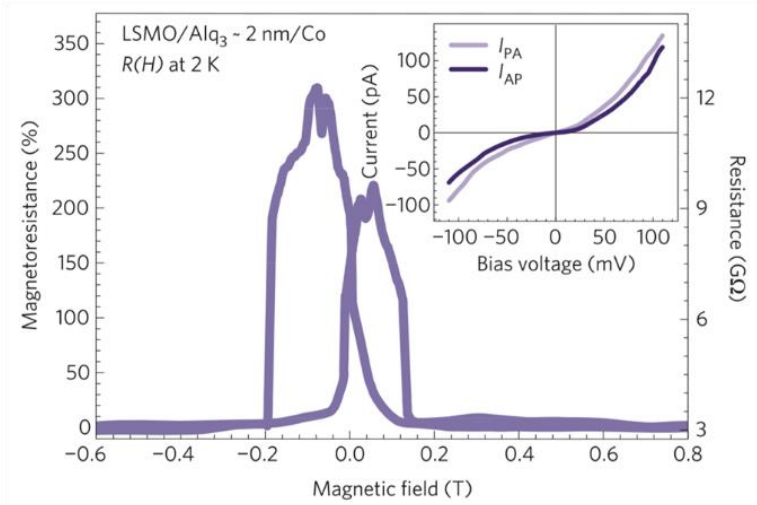

Figure 2.4 Nano-sized organic spin valve. a, Schematic demonstration of the nano-sized organic spin valve. Ultra-thin layer of organic semiconductor, $\mathrm{Alq}_{3}$ is sandwiched between LSMO and Co electrodes. The fabrication method is given in the text. $\mathbf{b}$, Magnetoresistance of LSMO/Alq $3(\sim 2 \mathrm{~nm}) / \mathrm{Co}$ nanosized spin valve device recorded at $2 \mathrm{~K}$ and $-5 \mathrm{mV}$. Co electrode has higher coercive field compared to LSMO electrode. The inset shows the $I-V$ characteristics of the device when the magnetisations of the ferromagnetic electrodes align parallel $\left(I_{\mathrm{PA}}\right)$ and antiparallel $\left(I_{\mathrm{AP}}\right)$ at $2 \mathrm{~K}$. This figure is modified from [73]. 


\subsubsection{Non-local spin valves}

Following the successful experimental observation of non-local spin signal in all metallic lateral spin valve in 2001, non-local spin valve has emerged as a standard technique to probe spin injection and transport in a variety of materials including, metals, inorganic semiconductors and graphene [48], [49], [50], [51], [52], [53], [76]. Apart from being the protocol for studying spin dynamics in various materials, this device architecture is also important for potential high performance magnetic integrated circuit applications such as spin field effect transistor [77], [78]. Unlike conventional charge current based spintronics devices, the non-local spin valve device operation is based on charge current free, pure spin current. Pure spin current which is a flow of spin angular momentum can be generated electrically by injecting a charge current across a ferromagnet $(\mathrm{NiFe})$ and non-magnet $(\mathrm{Ag})$ and drawing this current to the left as shown in Fig 2.5a. This leads to isotropic diffusion of pure spin current where there is only pure spin current in the right-hand side direction from the ferromagnet (Fig 2.5a).

Fig $2.5 \mathrm{~b}$ shows a typical non-local spin valve device structure and measurement configuration for n-GaAs. A current is applied between ferromagnetic electrodes 1 and 3 (injection electrode) while non-local voltage is detected between ferromagnetic electrodes 4 (detection electrode) and 5. It is important to note that only electrode 3 and 4 need to be ferromagnetic since it is the spin current between these two electrodes that generates the nonlocal spin signal. Fig 2.6a illustrates the electrochemical potential distribution for spin-up and spin down electrons in the non-magnetic materials under spin injection from the ferromagnetic electrode. When the distance between the two inner ferromagnetic electrodes are within the spin diffusion length of the non-magnetic material, the spin injection from the injection electrode causes non-equilibrium electrochemical potential difference between spin-up and spin-down electrons (spin accumulation). Depending on the relative magnetisation orientation of the two ferromagnetic electrodes, the detector electrode probes the different electrochemical potential profiles as shown in Fig 2.6b. The different electrochemical potentials between parallel and antiparallel magnetisations alignment of ferromagnetic electrodes give rise to the non-local spin signal as shown in Fig 2.5c. The coercive field difference between the two ferromagnetic electrodes is commonly achieved by tuning the width of the ferromagnet. 
a

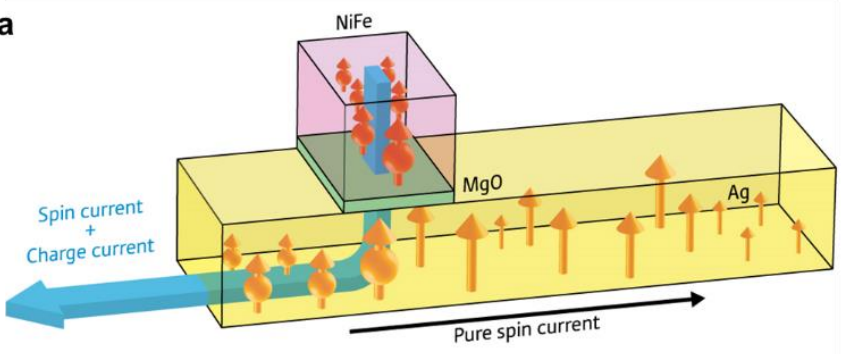

b

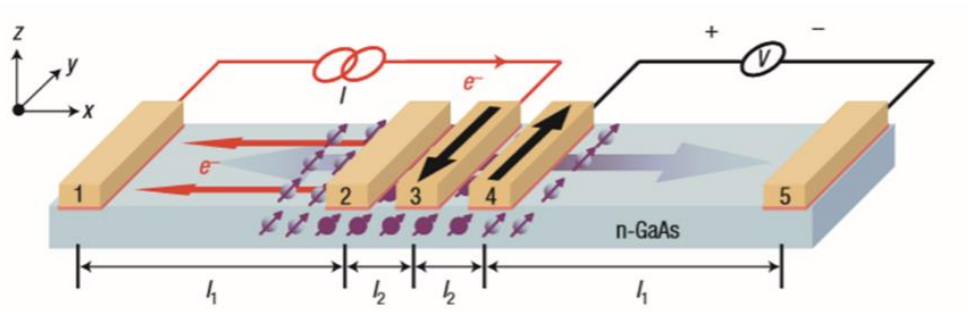

C

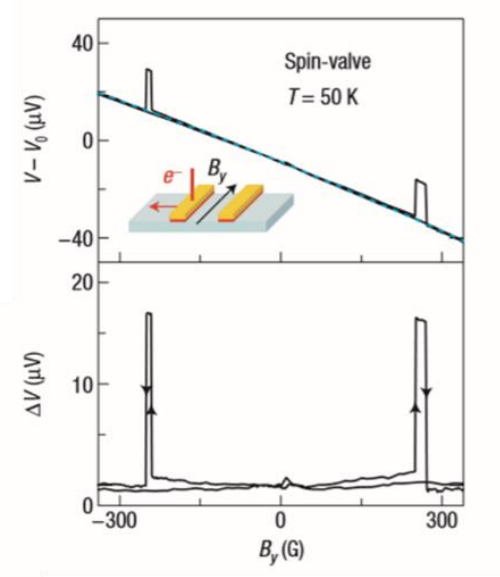

Figure 2.5 Non-local spin injection and detection. a, Schematic demonstration of electrical spin injection where electrical current is injected from the ferromagnet and directed to the left. Only pure spin current diffuses to the right. $\mathbf{b}$, Schematic illustration of a non-local spin valve device for $\mathrm{n}-\mathrm{GaAs}$. A current, $I$ is applied between electrodes 1 and 3 and non-local voltage, $V$ is detected between electrodes 4 and 5. The black arrows indicate the magnetisation orientation of the electrodes 3 and 4 . c, Detected non-local spin valve signal at $50 \mathrm{~K}\left(I_{1,3}=1 \mathrm{~mA}\right)$ where the external magnetic field is applied parallel to the ferromagnetic electrodes. This figure is modified from [79] and [48]. 
a

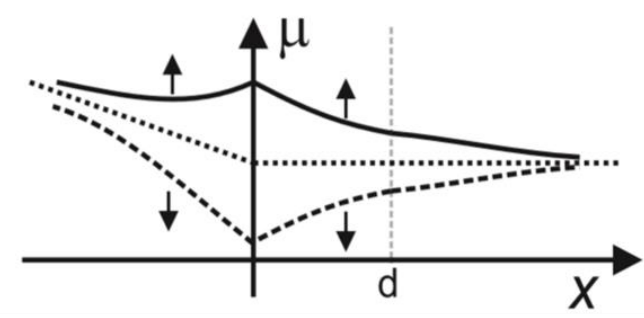

b

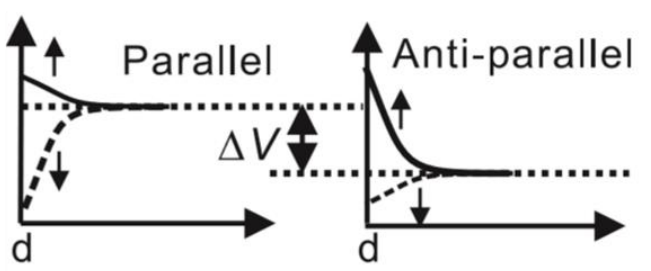

Figure 2.6 Spin accumulation in non-local spin valves. a, Electrochemical potential distribution in non-magnetic material under spin injection from ferromagnetic electrode. The up arrow indicates spin up electron whereas down arrow indicates spin down electrons. $X$ and $d$ denote the distance from ferromagnet and non-magnetic material interface and a distance from the ferromagnet/non-magnet interface that significant spin accumulation is present (much less than the spin diffusion length of the non-magnetic material), respectively. $\mathbf{b}$, The electrochemical potential in the ferromagnet detector. Parallel and antiparallel indicate the parallel and antiparallel magnetisation alignment orientation, respectively. $\Delta V$ represents the non-local voltage difference between parallel and antiparallel magnetisation configurations. This figure is modified from [80].

\subsubsection{Hanle effect}

The important litmus test for electrical spin injection in inorganic materials is the measurement of Hanle effect in perpendicular magnetic field. Hanle effect is based on magnetic field, $B$ induced spin precession with Larmor frequency, $\omega_{\mathrm{L}}$ where the magnetic field is perpendicular to the spin as illustrated in Fig 2.7a. Due to the spin precession, the spin accumulation is suppressed by the perpendicular magnetic field.

$$
\omega_{L}=\frac{g \mu_{B} B}{\hbar}
$$

where $g, \mu_{\mathrm{B}}, \hbar$ denote g-factor, Bohr magneton and Planck's constant divided by $2 \pi$. Nonlocal spin valves provide the ideal platform to study this effect where magnetic field is applied perpendicular to the ferromagnetic electrodes while performing the standard non-local spin valve measurement as shown in Fig 2.7b. Fig 2.7c shows the results of a standard Hanle type measurement using a non-local spin valve based on single layer graphene. The non-local resistance, $R_{\mathrm{NL}}$ is the resistance across the non-local arms of the device. $R_{\mathrm{NL}}$ as a function of 
perpendicular applied field can be fitted using Eq. (2.9) which depends on spin precession, spin diffusion and spin relaxation [49], [81]. $R_{\mathrm{NL}}$ for ferromagnetic aligned in parallel (antiparallel) orientation decays (increases) with the applied perpendicular magnetic field due to spin precession induced by the perpendicular magnetic field (Larmor precession).

$$
R_{N L} \propto \pm \int_{0}^{\infty} \frac{1}{\sqrt{4 \pi D t}} e^{\frac{-L^{2}}{4 D t}} \cos \left(\omega_{L} t\right) e^{\frac{-t}{\tau_{s}}} d t
$$

where $+(-)$ sign denotes parallel (antiparallel) magnetisations alignment of the ferromagnetic electrodes. $D, L, \tau_{\mathrm{s}}$ and $t$ denote diffusion constant, distance between the ferromagnetic electrodes, spin lifetime and diffusion time, respectively. The fitted parameters for $D$ and $\tau_{\mathrm{s}}$ allow independent estimation of spin diffusion length based on Einstein relation, $\lambda_{\mathrm{s}}=\sqrt{D \tau_{\mathrm{s}}}$ without the need for a gap dependence study of the spin signal. The spin lifetime extracted using the Hanle measurement should be considered as a lower bound of spin lifetime due to contact induced spin relaxation [82]. Spin accumulation, $\Delta \mu$ can be calculated using Eq. (2.10) by applying a known spin polarisation at interface value, $P[83]$.

$$
\Delta V=P \Delta \mu / 2
$$

where $\Delta V$ indicates the non-local voltage probed in non-local spin valve device as the applied perpendicular magnetic field is changed.
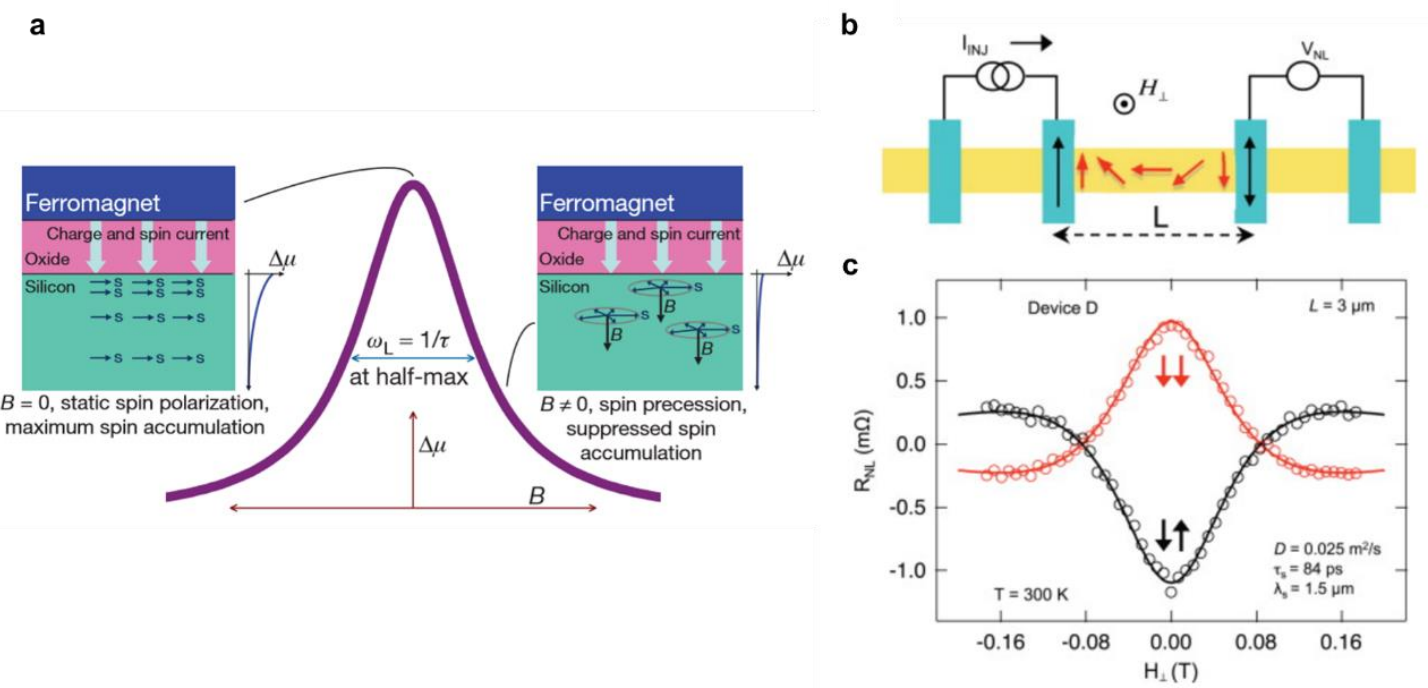

Figure 2.7 Hanle effect concept and experiments. a, Schematic illustration of Hanle effect where the net spin accumulation, $\Delta \mu$ is suppressed as a result of spin precession in magnetic field, $B$ applied perpendicular to the electron spins. b, Hanle Measurement using a non-local spin valve with magnetic field applied perpendicular to the ferromagnetic electrodes. The spin transport material is single layer graphene. c, Non-local resistance as a function of perpendicular applied magnetic field. The arrows 
indicate the relative magnetisation orientation of the ferromagnetic electrodes. This figure is modified from [83] and [81].

\subsection{Dynamical spin injection by spin pumping}

\subsubsection{Ferromagnetic resonance (FMR)}

When an external magnetic is applied to a ferromagnetic material, the magnetisation $(M)$ precesses around the effective magnetic field $\left(H_{\mathrm{eff}}\right)$ in the material. This precession motion is suppressed as magnetisation aligns with $H_{\text {eff. Ferromagnetic resonance occurs when a }}$ microwave is applied with frequency coinciding with the precession frequency. The magnetisation precession motion can be modelled by the Landau-Lifshitz-Gilbert equation [84], [85].

$$
\frac{d \mathbf{M}(t)}{d t}=-\gamma \mathbf{M}(t) \times \mathbf{H}_{\mathrm{eff}}+\frac{\alpha}{M_{s}} \mathbf{M}(t) \times \frac{d \mathbf{M}(t)}{d t}
$$

where $\mathbf{M}(t), \gamma, \alpha$ and $M_{\mathrm{s}}$ denote time-dependent magnetisation, gyromagnetic ratio, Gilbert damping constant and saturation magnetisation, respectively. The first term represents the precession motion whereas the second term accounts for damping. $\mathbf{H}_{\text {eff }}$ for soft ferromagnetic material such as Permalloy is a combination of external magnetic field $\mathbf{H}$ and static demagnetising field $\mathbf{H}_{\mathbf{M}}$ since the magnetocrystalline anisotropy is negligible $\left(\mathbf{H}_{\text {eff }}=\mathbf{H}+\mathbf{H}_{\mathbf{M}}\right)$ [85].

The external magnetic field angle, $\theta_{\mathrm{H}}$ can be related to the magnetisation angle, $\theta_{\mathrm{M}}$ [Eq. (2.12)] by considering the static equilibrium condition, $\mathbf{M} \times \mathbf{H}_{\text {eff }}=0$. Fig 2.9 provides a schematic definition of $\theta_{\mathrm{H}}$ and $\theta_{\mathrm{M}}[85]$.

$$
2 H \sin \left(\theta_{\mathrm{H}}-\theta_{\mathrm{M}}\right)+4 \pi M_{\mathrm{s}} \sin 2 \theta_{\mathrm{M}}=0
$$

The external ac field, $\mathbf{h}(t)$ creates a dynamic demagnetisation field $\mathbf{H}_{\mathrm{m}}(t)$ and dynamic magnetisation, $\mathbf{m}(t)$ such that the effective magnetic field is given by $\mathbf{H}_{\mathbf{e f f}}(t)=\mathbf{H}+\mathbf{H}_{\mathbf{M}}+\mathbf{H}_{\mathbf{m}}(t)$ $+\mathbf{h}(t)$ and the magnetisation is given by $\mathbf{M}(t)=\mathbf{M}+\mathbf{m}(t)$. By neglecting $\mathbf{h}(t)$ and assuming $\mathbf{m}(t)=\left(\mathrm{m}_{\mathrm{x}} \mathrm{e}^{\mathrm{i} \omega \mathrm{t}}, \mathrm{m}_{\mathrm{y}} \mathrm{e}^{\mathrm{i} \omega \mathrm{t}}, 0\right)$ around $\mathbf{M}$ as a solution to Eq. (2.11), the ferromagnetic resonance condition is given as [85],

$(\omega / \gamma)^{2}=\left[H_{\mathrm{FMR}} \cos \left(\theta_{\mathrm{H}}-\theta_{\mathrm{M}}\right)-4 \pi M_{\mathrm{S}} \cos 2 \theta_{\mathrm{M}}\right]\left[H_{\mathrm{FMR}} \cos \left(\theta_{\mathrm{H}}-\theta_{\mathrm{M}}\right)-4 \pi M_{\mathrm{s}} \cos \theta_{\mathrm{M}}^{2}\right]$ 
where $\omega$ and $H_{\mathrm{FMR}}$ are the angular frequency of magnetisation precession and ferromagnetic resonance external applied field, respectively. Note, $\omega=2 \pi f$, where $f$ is the microwave frequency. $4 \pi M_{\mathrm{s}}$ and $\omega / \gamma$ can be obtained by substituting $H_{\mathrm{FMR}}$ values at $\theta_{\mathrm{H}}=0^{\circ}$ and $90^{\circ}$ into Eq. (2.13). The $\theta_{\mathrm{H}}$ dependence of $\theta_{\mathrm{M}}$ is obtained by substituting $4 \pi M_{\mathrm{s}}$ and experimental $H_{\mathrm{FMR}}$ values into Eq. (2.12). Kittel's formula relates the $H_{\text {FMR }}$ with microwave frequency as shown in Eq. (2.14) [86].

$$
(\omega / \gamma)^{2}=H_{\mathrm{FMR}}\left(H_{\mathrm{FMR}}+4 \pi M_{\mathrm{S}}\right)
$$

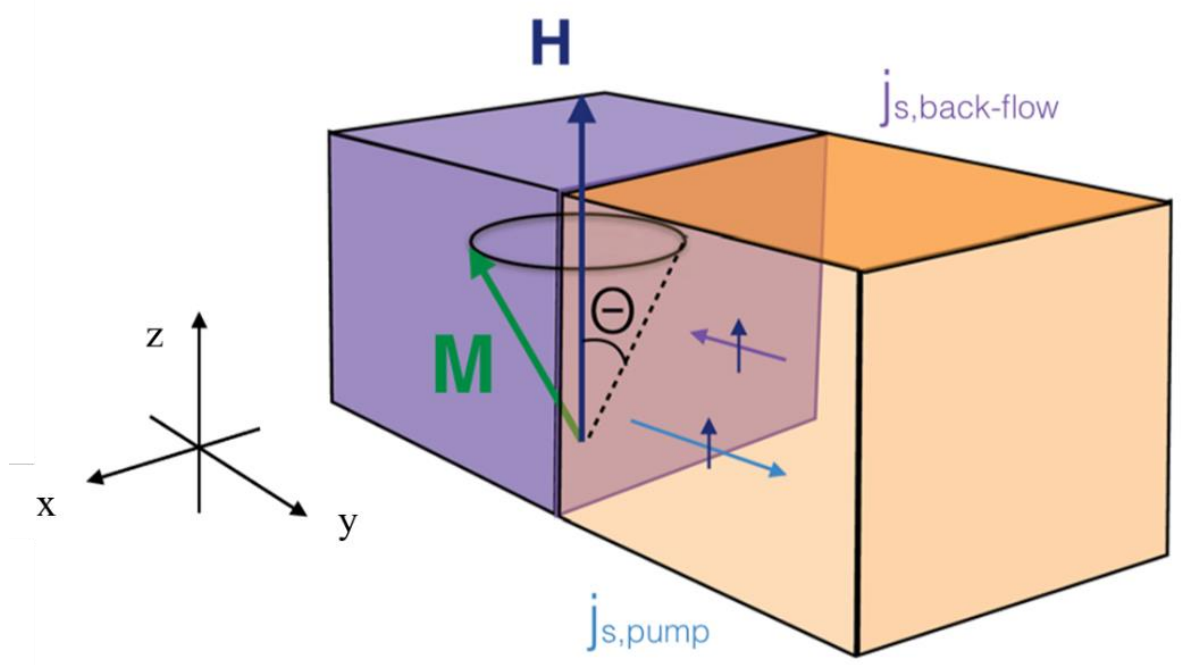

Figure 2.8 Spin pumping induced by FMR. Schematic illustration of pure spin current injection from ferromagnet (purple) to non-magnetic metal (orange) by FMR. H, M and $\theta$ denote external magnetic field, dynamic magnetisation and precession angle. $\mathrm{j}_{\mathrm{s} \text {,pump }}$ and $\mathrm{j}_{\mathrm{s} \text {,back-flow }}$ denote the "pumped" spin current and spin current back flow that depends on the thickness of non-magnetic metal. This figure is modified from [11].

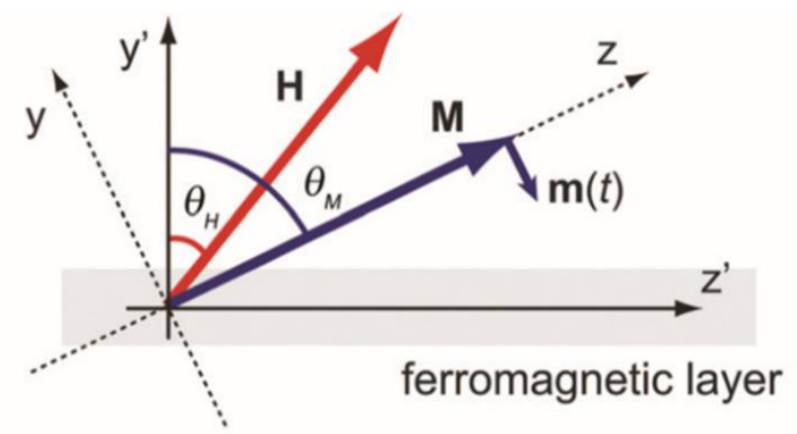

Figure 2.9 Schematic illustration of magnetic field and magnetisation angle definition. $H, M$ and $\mathbf{m}(t)$ denote external magnetic field, static magnetisation and dynamic magnetisation, respectively. $\theta_{\mathrm{H}}$ is the external magnetic angle and $\theta_{\mathrm{M}}$ is the magnetisation angle. This figure is taken from [85]. 


\subsubsection{Spin pumping induced by FMR}

When a non-magnetic metal or semiconductor (NM) is in contact with a ferromagnetic material (FM) under ferromagnetic resonance, a flow of pure spin current is pumped into the nonmagnetic material via exchange interaction at the interface. The dissipation of spin angular momentum from the ferromagnet to the non-magnetic material causes damping of the ferromagnetic resonance. This damping process creates a linewidth broadening of the ferromagnetic resonance line shape which is directly related to the Gilbert damping term in Eq. (2.11). FMR linewidth broadening is commonly used to measure the efficiency of spin injection across FM/NM interfaces. Tserkovnyak et al [87], [84] have derived the spin current, $\mathbf{I}_{\mathrm{s}}{ }^{\text {pump }}$ injected into a normal metal to be

$$
\boldsymbol{I}_{\mathrm{S}}^{\mathrm{pump}}=\frac{\hbar}{4 \pi}\left(A_{\mathrm{r}} \boldsymbol{m} \times \frac{d \boldsymbol{m}}{d t}-A_{\mathrm{i}} \frac{d \boldsymbol{m}}{d t}\right)
$$

where $\hbar$ and $\mathbf{m}$ represent the Planck's constant and the magnetisation direction, respectively. $A_{\mathrm{r}}$ and $A_{\mathrm{i}}$ are interface parameters that depends on both the reflection and transmission coefficient of spin-up and spin-down electrons. The details are provided in ref [88]. Theoretical $a b$ initio calculations show $A_{\mathrm{r}}$ can be approximated to be the real part of spin mixing conductance. This can be approximated by the number of conducting channels in the diffusive regime [11].

$$
A_{\mathrm{r}} \approx \operatorname{Re}\left[g^{\uparrow \downarrow}\right]=\frac{k_{\mathrm{F}}^{2}}{4 \pi} \approx \frac{1}{4 \pi}\left(3 \pi^{2} n\right)^{\frac{2}{3}}
$$

where $k_{\mathrm{F}}$ and $n$ denote the Fermi wave vector and electron density in the normal metal, respectively. Eq. (2.15) can therefore be further simplified to Eq. (2.17) when the ferromagnetic layer is thicker than the ferromagnetic coherence length.

$$
\boldsymbol{I}_{\mathrm{s}}^{\mathrm{pump}}=\frac{\hbar}{4 \pi} g_{\mathrm{r}}{ }^{\uparrow \downarrow} \boldsymbol{m} \times \frac{d \boldsymbol{m}}{d t}
$$

where $g_{\mathrm{r}}{ }^{\uparrow}$ indicates the real part of spin mixing conductance. The dc component of spin current density, $j_{\mathrm{s}}$ can be expressed as [85]

$$
j_{S}=\frac{\omega}{4 \pi} \int_{0}^{2 \pi / \omega} g_{\mathrm{r}}{ }^{\uparrow \downarrow} \frac{\hbar}{4 \pi M_{\mathrm{s}}{ }^{2}}\left[\boldsymbol{m} \times \frac{d \boldsymbol{m}}{d t}\right]_{\mathrm{z}} d t
$$

where $\mathrm{z}$ indicates the direction of magnetisation precession axis. When the normal metal acts as a perfect spin sink, the spin current injected across the F/NM is governed purely by the spin 
mixing conductance. However, if the thickness of NM is on the order of its spin diffusion length, a spin accumulation, $\mu_{\mathrm{s}}=\mu_{\uparrow}-\mu_{\downarrow}$ is created in the NM due to the injected spin current. This spin accumulation results in a back-flow of spin current as illustrated in Fig 2.8. The spin accumulation in the NM is given by Eq. (2.19) along with boundary conditions (a) and (b) [11].

(a) $y=0: \partial_{\mathrm{y}} \mu_{\mathrm{s}}=-\frac{4 e^{2} \rho}{\hbar} j_{s, 0}$

$$
\frac{d \mu_{\mathrm{s}}}{d t}=D \partial_{\mathrm{y}}^{2} \mu_{\mathrm{s}}-\frac{\mu_{\mathrm{s}}}{\tau_{\mathrm{sf}}}
$$

(b) $y=t_{\mathrm{NM}}: \partial_{\mathrm{y}} \mu_{\mathrm{s}}=0$

where $\rho, \tau_{\mathrm{sf}}$ and $t_{\mathrm{NM}}$ denote resistance, spin flip time and thickness of NM, respectively. $\mathrm{y}$ refers to the direction of spin current (perpendicular to the film plane) as illustrated in Fig 2.8. The spin accumulation induced spin current back-flow results in an effective reduction in the spin current injection across the interface by a back-flow factor, $\beta$ [11].

$$
\beta=\frac{\tau_{\mathrm{sf}} \delta_{\mathrm{sd}} / h}{\tanh \left(t_{\mathrm{NM}} / \lambda_{\mathrm{sd}}\right)}
$$

where $\lambda_{\text {sd }}$ and $\delta_{\text {sd }}$ represent spin diffusion length of NM and effective spin-flip scattering energy, respectively.

The effective spin mixing conductance, $g_{\mathrm{eff}}{ }^{\uparrow \downarrow}$ takes into account of this back-flow factor and this represents the actual spin injection efficiency across FM/NM interface [11].

$$
g_{\mathrm{eff}}^{\uparrow \downarrow}=g_{\mathrm{r}}^{\uparrow \downarrow} \frac{1}{1+\beta g_{\mathrm{r}}{ }^{\uparrow \downarrow}}
$$

The spin current decays from the FM/NM interface in the NM due to spin diffusion and spinflip scattering. The spin current density at a distance away from the FM/NM interface, $j_{\mathrm{s}}$ can be derived to be

$$
j_{s}=j_{s, 0} \frac{\sinh \left[\frac{t_{\mathrm{NM}}-y}{\lambda_{\mathrm{sd}}}\right]}{\sinh \left(t_{\mathrm{NM}} / \lambda_{\mathrm{sd}}\right)}
$$

where $j_{\mathrm{s}, 0}$ and $y$ denote the spin current density at the FM/NM interface and distance from the FM/NM interface (perpendicular to the film plane), respectively [85]. 


\subsubsection{Spin Hall effect (SHE) and Inverse spin Hall effect (ISHE)}

The observation of spin hall effect (SHE) enables the generation of pure spin current using charge current without external magnetic field in non-magnetic materials as shown in Fig 2.10a. SHE relies on the spin-orbit coupling (SOC) of the material to convert charge current to spin current where SOC generally scales with atomic number, $Z$ (SOC $\propto Z^{4}$ ) [89]. SHE was first observed experimentally in GaAs and InGaAs by Kato et al using magnetic optical Kerr effect (MOKE) [90]. The experimental verification of SHE triggered intense research on both fundamental study of SHE and application of SHE in novel spintronics devices. SHE can be classified into two regimes, intrinsic and extrinsic. The intrinsic SHE depends solely on the spin-orbit coupling effect without any impurity scattering process whereas extrinsic SHE depends on scattering processes at the impurity sites under spin orbit coupling effect. The indepth mechanisms responsible for both intrinsic and extrinsic SHE are beyond the scope of this thesis but can be found in [11], [91].

In contrast, the inverse spin hall effect (ISHE) is reciprocal of the SHE where spin current is converted into a charge current via spin-orbit interaction as illustrated in Fig 2.10b. SHE together with ISHE have enabled the generation and detection of pure spin current using nonmagnetic materials which allows novel non-magnetic materials to be considered for active elements in high performance spintronics devices. The charge current generated by ISHE, $\mathbf{J}_{\mathbf{c}}$ can be express as

$$
\mathbf{J}_{\mathrm{c}}=\theta_{\mathrm{SHE}}\left(\frac{2 e}{\hbar}\right) \mathbf{J}_{\mathrm{s}} \times \boldsymbol{\sigma}
$$

where $\theta_{\text {SHE }}$ is spin Hall angle which represents the material specific conversion efficiency between spin current and charge current. $\mathbf{J}_{\mathrm{s}}$ and $\boldsymbol{\sigma}$ denote spin current and spin polarisation vector, respectively. The spatial relationship between $\mathbf{J}_{\mathrm{c}}, \mathbf{J}_{\mathrm{s}}$ and $\boldsymbol{\sigma}$ is illustrated in Fig $2.10 \mathrm{~b}$. Table 2.1 provides the spin diffusion lengths and spin Hall angles for some commonly used materials for spintronics research. 

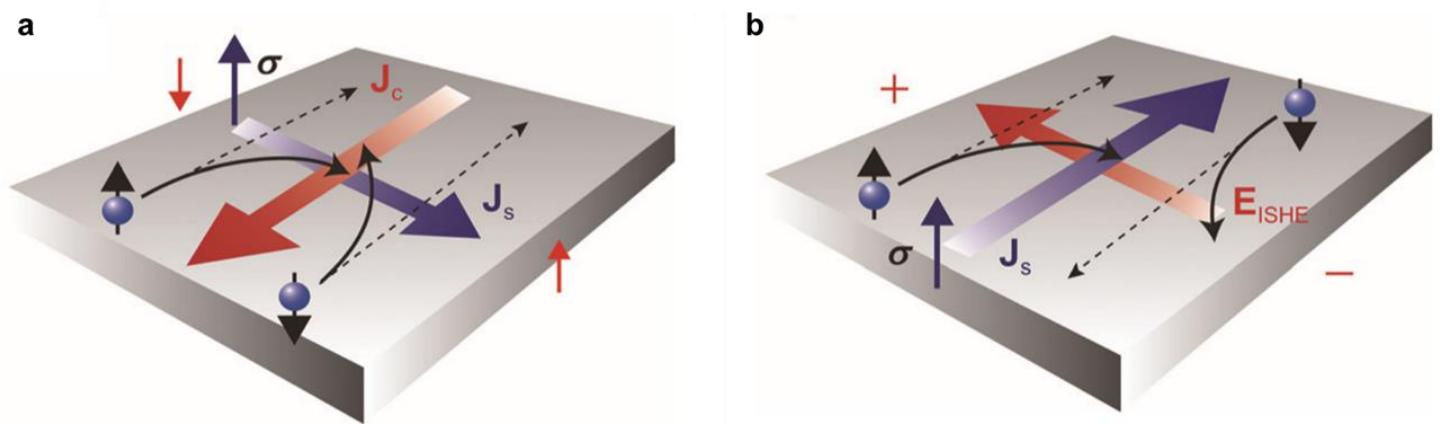

Figure 2.10 Schematic illustrations of spin Hall effect and inverse spin Hall effect. a, Spin Hall effect-conversion of charge current to spin current. b, Inverse spin Hall effect-conversion of spin current to charge current. $\mathbf{J}_{\mathrm{c}}, \mathbf{J}_{\mathrm{s}}, \boldsymbol{\sigma}$ and $\mathbf{E}_{\text {ISHE }}$ denote a charge current, a flow of pure spin current, spin polarisation vector of the spin current and the electric field generated by ISHE. This figure is modified from [85].

Table 2.1 Spin diffusion length and spin hall angles of commonly used materials in spintronics.

\begin{tabular}{lll}
\hline Material & Spin diffusion length $(\mathrm{nm})$ & Spin Hall angle (\%) \\
& & \\
\hline $\mathrm{Al}$ & $705 \pm 30$ & $0.032 \pm 0.006$ at $4.2 \mathrm{~K} \mathrm{[92]} \mathrm{[93]}$ \\
$\mathrm{Ag}$ & 700 & $0.7 \pm 0.1$ at $300 \mathrm{~K} \mathrm{[94]}$ \\
$\mathrm{Au}$ & 35 & $1.1 \pm 0.3$ at $300 \mathrm{~K}[95]$ \\
$\mathrm{Cu}$ & 500 & $0.32 \pm 0.03$ at $300 \mathrm{~K}[94]$ \\
$\mathrm{Pt}$ & 7.3 & $10 \pm 1$ at $300 \mathrm{~K}[94]$ \\
$\mathrm{Ta}$ & 1.9 & $-7.1 \pm 0.6$ at $300 \mathrm{~K}[94]$ \\
& & \\
\hline
\end{tabular}

\subsubsection{Probing spin transport using FMR and ISHE}

Saitoh et al reported successful spin current detection using ISHE in Py/Pt bilayer system in 2006 where Py is driven into FMR and the pure spin current injected into Pt is converted into a measurable charge current by ISHE [56]. The combination of FMR and ISHE has opened up a new avenue to study spin transport and spin to charge conversion in a wide variety of material systems. One of the most important advantages for using FMR as a spin injection method is that there is no net charge current flow associated with spin current injection. Thus, the spin current injected across FM/NM interface is not limited by the conductance mismatch problem [60]. As a result, the combination of FMR and ISHE has become a popular approach to study the spin transport in a range of semiconducting materials that would otherwise be challenging to study using conventional electrical spin injection techniques. 
Consider a metallic bilayer sample with a ferromagnet film in contact with a non-magnetic film with strong spin-orbit coupling. When the ferromagnet is excited into FMR, pure spin current is injected into the non-magnetic material and the ISHE generated current can be measured as a DC voltage along $\mathrm{x}$ axis as defined by ISHE symmetry (Fig 2.8). The FMR linewidth of ferromagnet can be used to estimate the effective spin mixing conductance across the $\mathrm{FM} / \mathrm{NM}$ interface

$$
g_{\mathrm{eff}}^{\uparrow \downarrow}=\frac{2 \sqrt{3} M_{\mathrm{s}} \gamma d_{\mathrm{F}}}{g \mu_{\mathrm{B}} \omega}\left(W_{\overline{\mathrm{F}}}-W_{\mathrm{F}}\right)
$$

where $d_{\mathrm{F}}, W_{\frac{\mathrm{F}}{\mathrm{N}}}$ and $W_{\mathrm{F}}$ denote thickness of ferromagnet, FMR linewidth of the FM/NM bilayer and FMR linewidth of ferromagnet, respectively [85]. Using Eq. (2.18) and expressions for dynamic component of magnetisation, an expression for the spin current density at the $\mathrm{FM} / \mathrm{NM}$ interface, $j_{\mathrm{S}}{ }^{\mathrm{F} / \mathrm{N}}$ can be derived to be

$$
j_{\mathrm{S}}^{\mathrm{F} / \mathrm{N}}=\frac{g_{\mathrm{eff}}{ }^{\uparrow \downarrow} \gamma^{2} h^{2} \hbar\left[4 \pi M_{\mathrm{S}} \gamma+\sqrt{\left(4 \pi M_{\mathrm{S}}\right)^{2} \gamma^{2}+4 \omega^{2}}\right]}{8 \pi \alpha^{2}\left[\left(4 \pi M_{\mathrm{S}}\right)^{2} \gamma^{2}+4 \omega^{2}\right]}
$$

The spin current is converted into a charge current via ISHE, the average charge current density, $\left\langle j_{\mathrm{c}}\right\rangle$ can be obtained by considering Eq. (2.22) [56], [85].

$$
\left\langle j_{\mathrm{c}}\right\rangle=\left(\frac{1}{d_{\mathrm{N}}}\right) \int_{0}^{d_{\mathrm{N}}} j_{\mathrm{c}}(y) d y=\theta_{\mathrm{SHE}}\left(\frac{2 e}{\hbar}\right) \frac{\lambda_{\mathrm{N}}}{d_{\mathrm{N}}} \tanh \left(\frac{d_{\mathrm{N}}}{2 \lambda_{\mathrm{N}}}\right) j_{S}^{F / N}
$$

where $d_{\mathrm{N}}$ is the thickness of the non-magnetic material. When the ferromagnet is also conductive, the ISHE generated charge current flows through the combined resistance circuit of ferromagnet and non-magnet layers in parallel. The ISHE voltage generated, $V_{\text {ISHE }}$ is given by

$$
V_{\mathrm{ISHE}}=\frac{w \theta_{\mathrm{SHE}} \lambda_{\mathrm{N}} \tanh \left(\frac{d_{\mathrm{N}}}{2 \lambda_{\mathrm{N}}}\right)}{d_{\mathrm{N}} \sigma_{\mathrm{N}}+d_{\mathrm{F}} \sigma_{\mathrm{F}}}\left(\frac{2 e}{\hbar}\right) j_{S}^{F / N}
$$

where $w$ and $d_{\mathrm{F}}$ denote width and thickness of the ferromagnetic material, respectively. The width of the ferromagnet is defined as the width of the ferromagnet parallel to the $V_{\text {ISHE. }} \sigma_{\mathrm{N}}$ and $\sigma_{\mathrm{F}}$ indicate the conductivity of non-magnet and ferromagnet, respectively [85].

Shikoh et al have taken a step forward to study the spin transport in Silicon in a lateral direction using a combination of FMR and ISHE as shown in Fig 2.11 [96]. In this experiment, Py injects pure spin current in p-Si when it is excited into FMR (spin pumping). The injected 
spin current diffuses in p-Si and undergoes spin-flip scattering within the semiconductor (spin transport). The spin current that arrives at the Pd wire positioned within spin diffusion length of p-Si next to Py box is converted into a detectable charge current via ISHE (Fig 2.11a). Fig $2.11 \mathrm{~b}$ shows an optical image of the gap spacing between Py box and Pd wire, this submicron gap spacing is achieved by two steps e-beam lithography. Fig 2.11c shows a typical FMR signal along with the detected ISHE signal at the Pd wire. In the presence of other voltage contributions such as anomalous Hall effect in Py in addition to the ISHE generated voltage, the ISHE contribution in the Pd wire can be extracted by fitting the field dependence of the electromotive force with both a symmetric $V_{\text {ISHE }}$ and asymmetric $V_{\text {asym }}$ Lorentzian functions as below

$$
V(H)=V_{\mathrm{ISHE}} \frac{\Gamma^{2}}{\mu_{0}^{2}\left(H-H_{\mathrm{FMR}}\right)^{2}+\Gamma^{2}}+V_{\mathrm{asym}} \frac{-2 \Gamma \mu_{0}\left(H-H_{\mathrm{FMR}}\right)}{\mu_{0}^{2}\left(H-H_{\mathrm{FMR}}\right)^{2}+\Gamma^{2}}
$$

where $\Gamma$ is the full width at half maximum FMR linewidth and $\mu_{0} H_{\mathrm{FMR}}$ is the Py FMR field. A microwave irradiation induced constant offset voltage was substracted from the measured voltage [56]. 


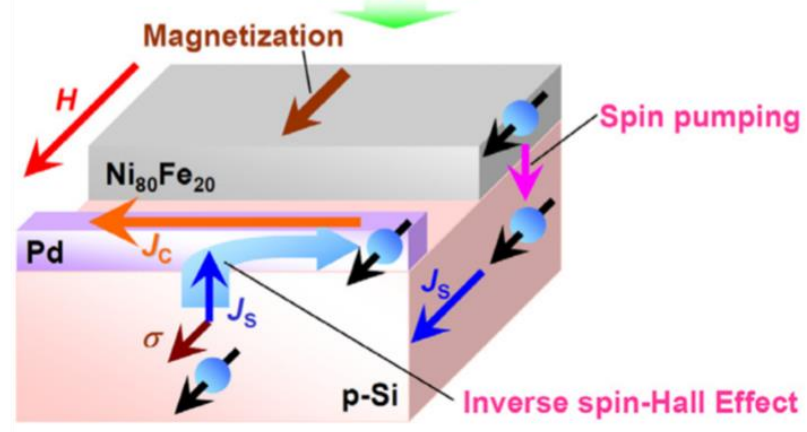

b

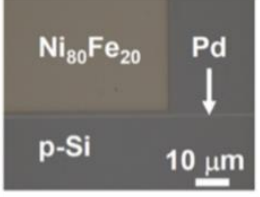

C

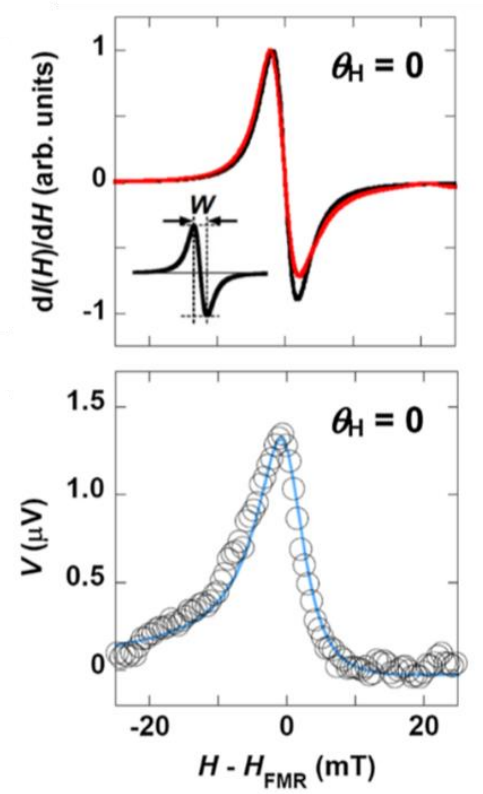

Figure 2.11 Lateral spin pumping and ISHE detection in p-Si. a, Schematic illustration of spin pumping in p-Si and ISHE detection in a lateral geometry. Spin pumping generates pure spin current in $\mathrm{p}$-Si and this spin current diffuses in the semiconductor. Spin current arrives at Pd wire is converted into a charge current via ISHE. b, Optical micrograph of active region of the device. c, Field $H$ dependence of FMR signals $d I(H) / d H$ measured for bare $\mathrm{Py} / \mathrm{SiO}_{2}$ (black line) and for $\mathrm{Py} / \mathrm{p}-\mathrm{Si} / \mathrm{Pd}$ (red line) (top figure). $I$ is the microwave absorption intensity and $W$ is the FMR linewidth (defined in the inset). Field $H$ dependence of voltage across the Pd wire $V$ (bottom figure). The magnetic field is applied parallel to the Py film $\left(\theta_{\mathrm{H}}=0\right)$. This figure is modified from [96].

Ohshima et al have demonstrated ISHE signal sign reversal when replacing Pt with Ta (Ta has opposite spin Hall angle to $\mathrm{Pt}$ ) for LAO/STO 2DEG system which provides conclusive evidence for spin transport in the 2DEG material (Fig 2.12a-b) [97]. The lateral spin pumping technique can be used to study the spin diffusion length of the spin transport material by two methods. The first method makes use of established spin diffusion model and fundamental spin pumping theory to estimate the spin diffusion length of the spin transport medium (fixed spin injector and detector spacing), the details are provided in [96] [97] [98] [99]. The drawbacks of this approach include uncertainty of spin-mixing conductance at the interface between FM/NM and spin Hall angle of the detector electrode as well as rough assumption about the portion of the injected spin current density that arrives at the detector electrode. Hence, it is difficult to extract an accurate spin diffusion length based on this method. The second method fits an exponential decay function to the ISHE generated current, $I_{\text {norm }}$ as a function of gap spacing between Py and Pt, $L_{\mathrm{Py}-\mathrm{Pt}}$ as illustrated in Fig 2.12c. Spin diffusion length, $\lambda_{\mathrm{s}}$ can be 
extracted from the fitting

$$
I_{\text {norm }}\left(L_{\mathrm{Py}-\mathrm{Pt}}\right)=a e^{-L_{\mathrm{Py}-\mathrm{Pt}} / \lambda_{\mathrm{s}}}
$$

where $a$ is a constant. Even though this approach requires fabrication of multiple devices, it provides a straightforward method for evaluation of spin diffusion length of different materials. Table 2.2 compares the spin diffusion lengths of various materials extracted using lateral spin pumping and non-local spin valve experiments. The spin diffusion lengths obtained by the two methods are generally comparable to each other.
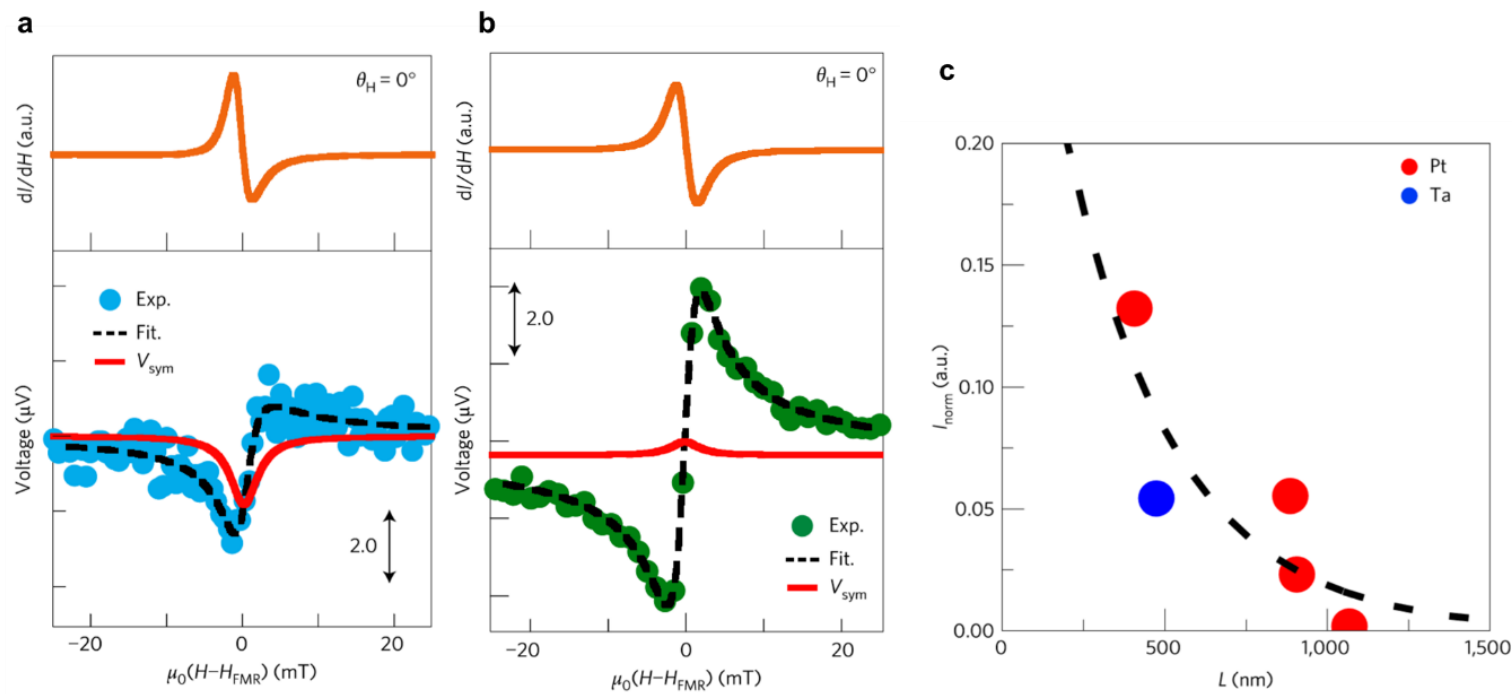

Figure 2.12 Lateral spin pumping control experiment and spin diffusion length extraction. a, Field dependence of FMR signal and voltage probed across $\mathrm{Pt}$ for $\mathrm{Py} /(\mathrm{LAO} / \mathrm{STO}) / \mathrm{Pt}$ device. b, Field dependence of FMR signal and voltage probed across Ta for Py/(LAO/STO)/Ta. The magnetic field is applied parallel to Py film for both Pt and Ta devices $\left(\theta_{\mathrm{H}}=0\right)$. c, Gap spacing between Py and Pt $L$ dependence of ISHE induced current $I_{\text {norm }}=V_{\text {ISHE }} / R$ The black dashed line is an exponential fit. This figure is modified from [97].

Table 2.2 Comparison between the spin diffusion lengths of various materials estimated by lateral spin pumping and conventional four terminal non-local spin valve techniques.

\begin{tabular}{|c|c|c|}
\hline Material & $\begin{array}{l}\text { Spin diffusion length from } \\
\text { lateral spin pumping (nm) }\end{array}$ & $\begin{array}{l}\text { Spin diffusion length non-local } \\
\text { spin valve }(\mathrm{nm})\end{array}$ \\
\hline n-Ge & $660 \pm 200$ at $300 \mathrm{~K}$ [98] & 580 at $4 \mathrm{~K}$ [51] \\
\hline LAO/STO 2DEG & $337 \pm 181$ at $300 \mathrm{~K}$ [97] & \\
\hline $\mathrm{p}-\mathrm{Si}$ & 148 at $300 \mathrm{~K}[96]$ & \\
\hline n-GaAs & 1090 at $300 \mathrm{~K}$ [100] & 1600 at $205 \mathrm{~K}$ [52] \\
\hline $\mathrm{Cu}$ & 270 at $300 \mathrm{~K}[101]$ & 350 at $300 \mathrm{~K}[68]$ \\
\hline Graphene SLG & 1360 at $300 \mathrm{~K}$ [99] & 1100 at $300 \mathrm{~K}[50]$ \\
\hline
\end{tabular}




\subsection{Spin relaxation mechanisms}

When an electron charge is injected into a system, it will remain in the system irrespective of time. In contrast, when a spin is injected into a non-magnetic material, it will eventually lose its spin information due to a number of relaxation mechanisms, i.e., spin dephasing is time dependent. There are two characteristic time scales associated with the spin decoherence processes, $T_{1}$ and $T_{2}$ are spin relaxation time and spin dephasing time, respectively. $T_{1}$ and $T_{2}$ can be defined by relating electronic spin magnetisation, $\mathbf{M}$, in an externally applied magnetic field, $\mathbf{B}(t)=B_{0} \widehat{\mathbf{z}}+\mathbf{B}_{1}(t)$, where $B_{0}$ is a static component of $\mathbf{B}(\mathrm{t})$ along the $\mathrm{z}$-axis and $\mathbf{B}_{1}(t)$ is a transverse oscillating magnetic field perpendicular to z-axis, as follows [102], [103],

$$
\begin{gathered}
\frac{\partial M_{x}}{\partial t}=\gamma(\mathbf{M} \times \mathbf{B})_{x}-\frac{M_{x}}{T_{2}}+D \nabla^{2} M_{x} \\
\frac{\partial M_{y}}{\partial t}=\gamma(\mathbf{M} \times \mathbf{B})_{y}-\frac{M_{y}}{T_{2}}+D \nabla^{2} M_{y} \\
\frac{\partial M_{z}}{\partial t}=\gamma(\mathbf{M} \times \mathbf{B})_{z}-\frac{M_{z}-M_{z}{ }^{0}}{T_{1}}+D \nabla^{2} M_{z}
\end{gathered}
$$

where $\gamma$ is the electron gyromagnetic ratio, $\gamma=\mu_{\mathrm{B}} \mathrm{g} / \hbar$, $\mathrm{D}$ is the diffusion constant (scalar for isotropic system and tensor for anisotropic system) and $M_{z}{ }^{0}=\chi B_{0}$ is the thermal equilibrium magnetisation, where $\chi$ denotes the static magnetic susceptibility. $T_{1}$ is only involved in the time evolution of $M_{\mathrm{z}}$ [Eq. (2.32)] and therefore represents the time for longitudinal magnetisation to reach equilibrium. This process requires energy dissipation from the spin system to the lattice via phonons. In contrast, $T_{2}$ is involved in the time evolution of $M_{\mathrm{x}}$ and $M_{\mathrm{y}}$ [Eqs. (2.30) and (2.31)] which determines the time for an ensemble transverse spins precessing in phase to lose their phase coherence due to spatial and temporal fluctuations of the precession frequencies. In addition, for localised electrons such as donor states in semiconductors, a spatial inhomogeneity in the field causes a reversible phase loss that is known to affect $T_{2}$ [104]. A spin-echo experiment can be used to eliminate reversible phase losses and, in this experiment, $T_{2}{ }^{*}$ defines the ensemble spin dephasing time and $T_{2}$ is reserved for ensemble spin dephasing time due to irreversible processes only where $T_{2}{ }^{*} \leq T_{2}\left(T_{2}{ }^{*}=T_{2}\right.$ for conduction electrons) [105]. In isotropic and cubic materials, $T_{1}=T_{2}$ if $\gamma B_{0} \ll 1 / \tau_{\mathrm{c}}$, where $\tau_{\mathrm{c}}$ is the correlation or interaction time. $1 / \tau_{\mathrm{c}}$ is the rate of change of effective magnetic field that causes spin dephasing. There is an on-going debate about the dominant spin relaxation mechanism in organic materials. The following subsections will provide an overview of different spin relaxation mechanisms in nonmagnetic materials, starting from established Elliot-Yafet, Dyakonov-Perel and hyperfine interaction, and finish with exchange mediated spin transport proposed by $\mathrm{Z}$. Yu for organic 
materials.

\subsubsection{Elliot-Yafet spin relaxation mechanism}

The Elliot-Yafet (EY) spin relaxation is based on ordinary momentum scattering at phonons and impurities where spin-orbit coupling in the electron wave function is induced by the presence of ion impurities and/or other inversion symmetry breaking sources as illustrated in Fig 2.13a. The presence of spin-orbit coupling generates a potential $V_{\text {SO }}$ in addition to the periodic lattice potential $V_{\mathrm{SC}}[104]$,

$$
V_{\mathrm{SO}}=\frac{\hbar}{4 m^{2} c^{2}} \nabla V_{\mathrm{SC}} \times \widehat{\mathbf{p}} \cdot \widehat{\boldsymbol{\sigma}}
$$

where $\mathrm{m}, \widehat{\mathbf{p}}$ and $\widehat{\boldsymbol{\sigma}}$ denote free-electron mass, linear momentum operator $(\widehat{\mathbf{p}} \equiv-i \hbar \nabla)$ and Pauli spin matrices. Under the influence of spin-orbit interaction, single electron Bloch wave functions in a solid are not the eigenstates of $\widehat{\sigma}_{z}$ but a combination of Pauli spin up $(|\uparrow\rangle)$ and down $(|\downarrow\rangle)$ states. For a solid that possesses a centre of symmetry such as elementary metals which Elliot-Yafet spin relaxation is considered, the spin-up and spin-down electrons Bloch states can be express as,

$$
\begin{gathered}
\boldsymbol{\Psi}_{\mathbf{k} n \uparrow}(\mathbf{r})=\left[a_{\mathbf{k} n}(\mathbf{r})|\uparrow\rangle+b_{\mathbf{k} n}(\mathbf{r})|\downarrow\rangle\right] e^{i \mathbf{k} \cdot \mathbf{r}} \\
\Psi_{\mathbf{k} n \downarrow}(\mathbf{r})=\left[a_{-\mathbf{k} n}^{*}(\mathbf{r})|\downarrow\rangle-b_{-\mathbf{k} n}^{*}(\mathbf{r})|\uparrow\rangle\right] e^{i \mathbf{k} \cdot \mathbf{r}}
\end{gathered}
$$

where $\mathbf{k}, \mathbf{r}$ and $n$ denote lattice momentum, radius vector and band index, respectively. $a$ and $b$ are lattice periodic coefficients, in most cases, $|a|$ is near unity and $|b|$ is close to zero. Using perturbation theory, $V_{\text {SO }}$ is turned on which couples the electron states of opposite Pauli spins with the same $\mathbf{k}$ and different $n$, this gives

$$
|b| \approx \lambda_{\mathrm{SO}} / \Delta E \ll 1
$$

where $\Delta E$ is the energy difference between the bands and $\lambda_{\mathrm{So}}$ is the spin-orbit coupling strength. $|b|$ is close to zero since the spin-orbit coupling strength is normally much smaller than the energy difference between bands. Hence, for EY spin relaxation, spin-orbit coupling on its own cannot lead to spin relaxation. Spin relaxation occurs when spin-orbit coupling combines with momentum scattering that couples spin-up and spin-down states. Therefore, the spin relaxation time, $\tau_{\mathrm{s}}$ is proportional to the momentum scattering time, $\tau_{\mathrm{p}}$ for EY spin relaxation [106]. As a result, $\tau_{\mathrm{s}}$ usually becomes longer at low temperatures due to less frequent momentum scattering at low temperatures (phonon scattering reduces at low temperatures) 
[106], [107]. Assuming Boltzmann transport, charge carrier mobility, $\mu$ can be related to $\tau_{\mathrm{p}}$ through $\mu=\mathrm{e} \tau_{\mathrm{p}} / \mathrm{m}^{*}$ ( $\mathrm{m}^{*}$ is the effective mass of the charge carrier). From Einstein relation, $\tau_{\mathrm{s}}$ is directly proportional to the diffusion constant of charge carriers, $D$. EY spin relaxation mechanism has been observed experimentally in a number of metals and inorganic semiconductors such as Silicon [104], [108], [109], [110].
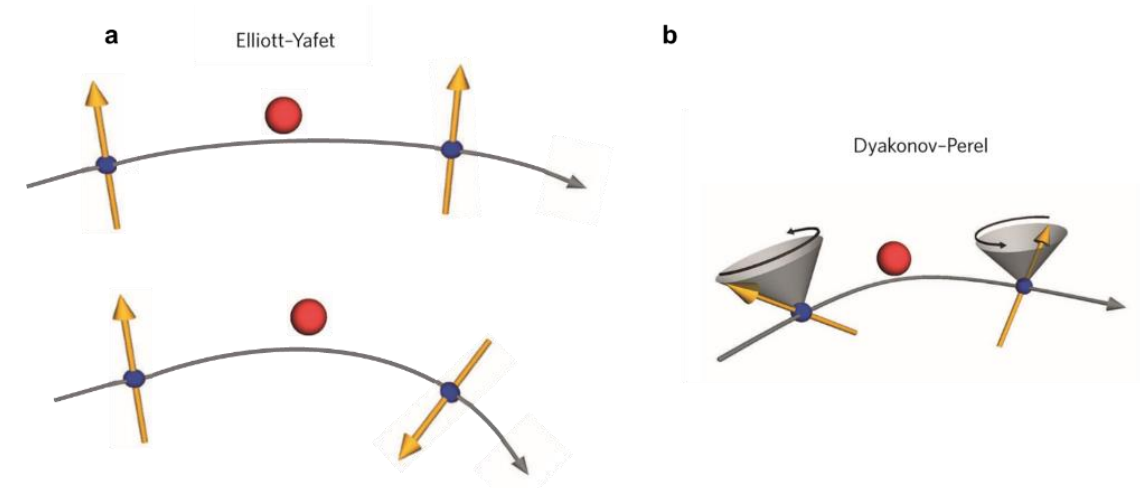

Figure 2.13 Schematic demonstration of Elliot-Yafet and Dyakonov-Perel spin relaxation mechanisms. a, Elliot-Yafet spin relaxation where electron spin (blue particle with arrow indicating spin direction) scatters by an impurity (red particle) causes spin flip. Top panel: Spin preserved (electron spin direction remained the same) after scattering off an impurity site. Bottom panel: Spin relaxed (electron spin direction flipped) after scattering off an impurity site. b, Dyakonov-Perel spin relaxation where spin precession angle changes due to change in effective magnetic field due to momentum scattering. This figure is modified from [82].

\subsubsection{Dyakonov-Perel spin relaxation mechanism}

Dyakonov-Perel (DP) spin relaxation occurs in systems lacking inversion symmetry such as GaAs where the inversion symmetry is broken by the presence of two different atoms in the lattice and other semiconductor heterostructures (two-dimensional electron gas). It is based on the electron spin precession travelling through fluctuating spin-orbit fields induced by spinorbit coupling where its precession angle changes after each momentum scattering event (spin precession occurs between scattering events) as illustrated in Fig 2.13b [82]. The spin-orbit field can either be Dresselhaus [111] or Rashba [112] type where in both cases the linear momentum state of an electron controls the effective magnetic field direction due to electron spin and linear momentum locking [113]. Based on the concept of motional narrowing, the spin-orbit fields, in effect, act as randomly fluctuating fields for moving spins. This essentially translates into increasing the electron scattering events, reduces its spin relaxation [104]. The correlation time for the fluctuation is given by the momentum scattering time, this gives the 
spin relaxation rate $1 / \tau_{\mathrm{s}} \approx \lambda_{\mathrm{SO}}{ }^{2} \tau_{\mathrm{p}}$ where $\lambda_{\mathrm{SO}}$ is magnitude of spin-orbit field [expressed in $\mathrm{Hz}$, Larmor frequency of the spin orbit field (in Tesla)] [82]. The inverse proportional relationship between spin relaxation time and momentum scattering time results in different temperature dependence of $\tau_{\mathrm{s}}$ compared to EY spin relaxation. DP spin relaxation has been experimentally observed in several III-V semiconductors such as GaAs and GaN, and n-doped GaAs/AlGaAs two-dimensional electron gas [114], [115], [116].

\subsubsection{Hyperfine interaction}

Hyperfine interaction (HFI) is based on the interaction between magnetic moment of electron spins and magnetic moment of nuclei which is important for spin relaxation of localised carriers such as, hopping mediated charge transport between localised states in organic semiconductors and carriers confined in quantum dots [104], [117]. The effective Hamiltonian for Hyperfine interaction is given by the Fermi contact potential energy. For an electron, $\mathbf{r}$ with spin, $\mathbf{S}$ [118],

$$
H=\frac{8 \pi}{3} \frac{\mu_{0}}{4 \pi} g_{0} \mu_{\mathrm{B}} \sum_{i} \hbar \gamma_{n, i} \mathbf{S} \cdot \mathbf{I}_{i} \delta\left(\mathbf{r}-\mathbf{R}_{i}\right)
$$

where $\mu_{0}, g_{0}$ and $\mu_{\mathrm{B}}$ denote the vacuum permeability, free electron g-factor (2.0023) and Bohr magneton, respectively. $\mathbf{I}_{i}, \mathbf{R}_{i}, \gamma_{n, i}$ denote nuclear spin, the position and nuclear gyromagnetic ratio, respectively where $\mathrm{i}$ is the label for nuclei. This results in the spin of electron with wavefunction $\psi(\mathrm{r})$ experiencing an effective magnetic field [104],

$$
\mathbf{B}_{n}=\frac{2 \mu_{0}}{3} \frac{g_{0}}{g} \sum_{i} \hbar \gamma_{n, i} \mathbf{I}_{i}\left|\psi\left(\mathbf{R}_{i}\right)\right|^{2}
$$

where $\mathrm{g}$ is the effective $\mathrm{g}$ factor of the electron. The random spatial variation in $\mathbf{B}_{n}$ leads to inhomogeneous dephasing of spin ensemble with spin relaxation rate, $1 / \tau_{\mathrm{s}} \propto \omega_{\mathrm{c}}{ }^{2} \tau_{\mathrm{c}}$ where $\omega_{\mathrm{c}}$ is the precession frequency of the electron spin in random nuclear fields and $\tau_{\mathrm{c}}$ is the correlation time for nuclear field fluctuation. The correlation time is given by the residence time of carrier on a localised site before moving to the next site [117]. Even though the HPI Hamiltonian is relatively weak due to the nuclear gyromagnetic ratio is orders of magnitude smaller than the electron gyromagnetic ratio, the Hyperfine interaction constant can be large for organic semiconductors due to the presence of many hydrogen nuclei. The Hyperfine interaction constant reduces when the delocalisation length of charge carrier in organic semiconductor increases since the delocalisation decreases the spin density at each nucleus [119]. For organic semiconductors, the Hyperfine model would expect a decrease in spin lifetime at low 
temperatures due to more localised charge transport at low temperatures for thermally activated hopping conduction.

The effect of Hyperfine interaction becomes negligible when the externally applied magnetic field exceeds $\mathbf{B}_{n}$ [104]. The effect of Hyperfine interaction on the spin physics in organic semiconductors is explored experimentally by a variety of techniques such as ODMR, OMAR and two terminal spin valves. Hyperfine interaction induced spin relaxation was observed in ODMR and two terminal spin valve measurements for standard and deuterated poly(dioctyloxy)phenylenevinylene (DOO-PPV) [30]. Moreover, the presence of OMAR is believed to originate mainly from Hyperfine interaction where general correlation between HFI strength and OMAR line-shape has been reported [120], [121], [122], [123]. However, the OMAR in $\mathrm{Alq}_{3}$ was not affected by deuteration which contradicts the HFI induced OMAR theory [124]. This opens up room for debate about the dominant mechanism for OMAR.

\subsubsection{Exchange mediated spin transport}

Z. Yu presented a theoretical study on the spin transport in organic semiconductor via exchange coupling between relatively localised carriers where this exchange coupling enables fast spin transport decoupled from slow charge transport in organic semiconductors without conductivity mismatch problem [125]. The exchange coupling between carriers originates from electron wave function overlap, the coupling constant, $\bar{J}$ for s electrons is given by [125]

$$
\bar{J}=0.821 \frac{e^{2}}{\epsilon \xi}\left(\frac{\bar{R}}{\xi}\right)^{\frac{5}{2}} e^{\left(\frac{-2 \bar{R}}{\xi}\right)}
$$

where $\epsilon, \xi$ and e denote the dielectric constant, the polaron localisation length and electron charge, respectively. $\bar{R}$ depends on the carrier density, $n$ (number of carriers per unit volume), $\bar{R}=n^{-1 / 3}$. In the presence of exchange coupling between carriers, the diffusion constant, $D$ consists of diffusion constant due to hopping, $D_{\mathrm{h}}$ and diffusion constant due to exchange coupling, $D_{\mathrm{e}}\left(D=D_{\mathrm{h}}+D_{\mathrm{e}}\right)$ [125]. Fig 2.14a shows the overall diffusion constant as a function of carrier concentration for $\mathrm{Alq}_{3}$ molecular system.

For carrier concentrations below $10^{17} \mathrm{~cm}^{-3}$, the diffusion constant comes purely from the hopping diffusion constant. When the carrier concentration exceeds $10^{17} \mathrm{~cm}^{-3}$, the diffusion constant increases rapidly due to the contribution from exchange diffusion constant (the higher the carrier concentration, the stronger the exchange coupling between the carriers due to the 
effective reduction in the distance between localised carriers).

$$
\begin{gathered}
D_{\mathrm{h}}=\frac{\mu k_{\mathrm{B}} T}{e} \\
D_{\mathrm{e}}=\sqrt{\frac{\pi}{12}} \frac{\bar{J} \bar{R}^{2}}{\hbar}
\end{gathered}
$$

In organic semiconductors, the spin relaxation rate, $\tau_{\mathrm{s}}^{-1}$ depends on the spin relaxation rate due to Hyperfine interaction, $\tau_{\mathrm{sh}}{ }^{-1}$ and spin-orbit coupling, $\tau_{\mathrm{ss}}{ }^{-1}\left(\tau_{\mathrm{s}}{ }^{-1}=\tau_{\mathrm{sh}}{ }^{-1}+\tau_{\mathrm{ss}}{ }^{-1}\right)$. The spin relaxation rate due to Hyperfine interaction is given by [117], [126]

$$
\tau_{\mathrm{sh}}{ }^{-1}=\frac{2}{3} \Omega_{\mathrm{h}}{ }^{2} \tau_{\mathrm{c}}
$$

where $\Omega_{\mathrm{h}}$ is the Larmor frequency of the local Hyperfine field and $\tau_{\mathrm{c}}$ is the correlation time which corresponds to the dwell time of a spin on a molecule before moving to the next molecule. The spin relaxation rate due to spin-orbit coupling is given by [127]

$$
\tau_{\mathrm{ss}}{ }^{-1}=2 \chi^{2} \tau_{\mathrm{c}}{ }^{-1}
$$

where $\chi^{2}$ is the spin mixing parameter due to spin-orbit coupling, the details are given in [127], [128]. Yu obtained this relationship by performing a rigorous density-matrix theory for organic semiconductors with hopping conduction. It is important to note that this spin-orbit coupling induced spin relaxation in organic semiconductor is in stark contrast to the ElliotYafet spin relaxation where the spin relaxation time decreases with increasing diffusion constant (opposite to the case of EY). This difference originates from the charge transport scattered by phonons in crystalline solids with band transport (EY spin relaxation) in contrast to polaron hopping conduction assisted by phonons. The full details can be found in [127]. The correlation time depends on both the hopping and exchange rate since both hopping and exchange lead to spin movement, and it is given by [125]

$$
\frac{1}{\tau_{\mathrm{c}}}=\frac{6 D_{\mathrm{h}}}{\bar{a}^{2}}+\frac{6 D_{\mathrm{e}}}{\bar{R}^{2}}
$$

where $\bar{a}$ is the average inter-molecule distance (lattice constant). The first term comes from hopping transport while the second term comes from exchange spin transport. This shows correlation time decreases when exchange is involved (spins move faster). As a result, the spin relaxation due to HFI will be reduced while the spin relaxation due to spin-orbit coupling will be enhanced when exchange is involved. At high carrier concentration where exchange spin 
transport is dominant, the spin relaxation due to HFI becomes negligible such that the spin relaxation is essentially controlled by spin-orbit interaction. Using Einstein relation, spin diffusion length can be obtained by [129]

$$
\lambda_{\mathrm{s}}=\sqrt{D \tau_{\mathrm{s}}}=\sqrt{D\left(\tau_{\mathrm{sh}}{ }^{-1}+\tau_{\mathrm{ss}}{ }^{-1}\right)^{-1}} \simeq \frac{\bar{R}}{2 \sqrt{3} \chi}
$$

When the exchange spin transport is dominant (high carrier concentration), the spin diffusion length is no longer sensitive to the diffusion constant but controlled mainly by the spin-orbit coupling strength (Fig 2.14b).
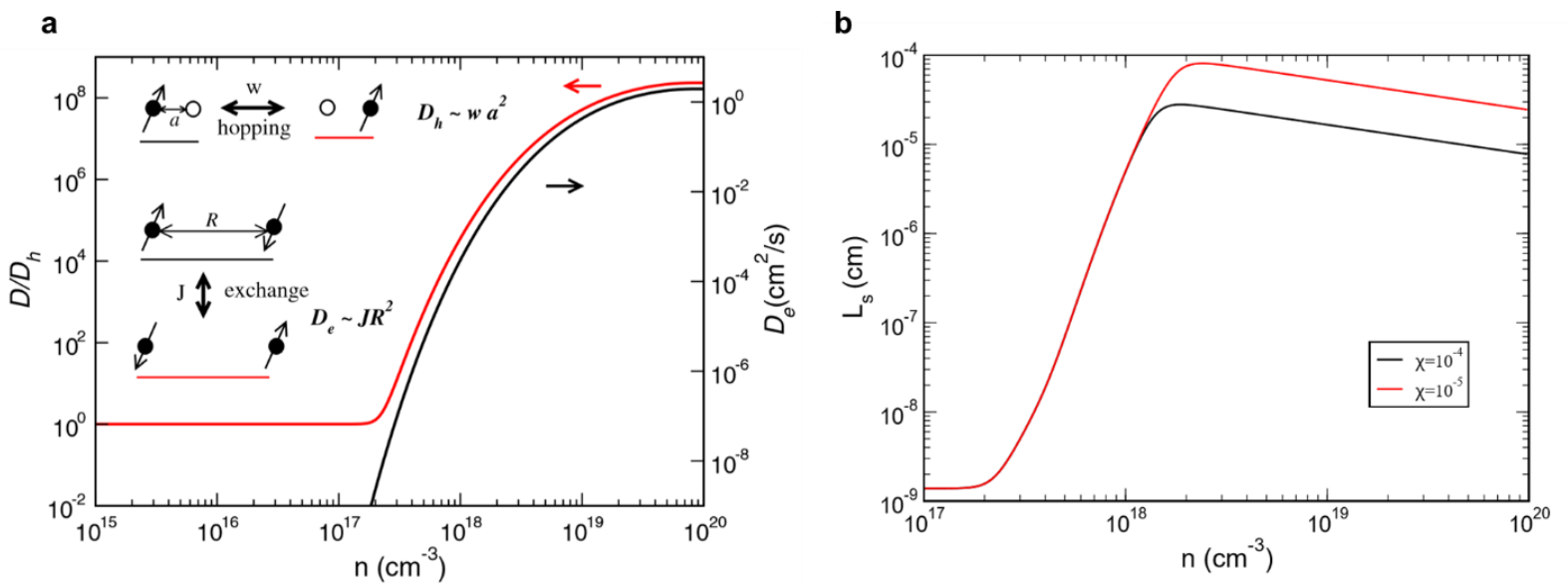

Figure 2.14 Exchange mediated spin transport. a, Spin diffusion constant as a function of carrier concentration for $\mathrm{Alq}_{3}$. The parameters used for simulation are $\epsilon=2, \xi=1 \mathrm{~nm}$, $T=100 \mathrm{~K}$ and $\mu=10^{-6} \mathrm{~cm}^{2} / \mathrm{Vs}$ [130]. The solid black line is for exchange spin diffusion constant, $D_{\mathrm{e}}$ whereas the solid red line is for $\left(D_{\mathrm{e}}+D_{\mathrm{h}}\right) / D_{\mathrm{h}}$ where $D_{\mathrm{h}}$ denotes hopping spin diffusion constant. $\mathbf{b}$, Spin diffusion length as a function of carrier concentration for $\mathrm{Alq}_{3}$. The black and red lines use spin-mixing parameters, $\chi^{2}=10^{-4}$ and $10^{-5}$, respectively. Organic material with stronger spin-orbit coupling has a higher value of $\chi^{2}$ which results in a lower value of spin diffusion length. The HFI field is $\Omega_{\mathrm{h}} / \gamma=1 \mathrm{mT}$ and $\bar{a}$ is $1.3 \mathrm{~nm}$ [127]. This figure is modified from [125]. 


\section{Chapter 3}

\section{Experimental methods}

\subsection{Organic materials}

Charge carriers in organic conjugated semiconductors are spin-1/2 polarons (holes in HOMO and electrons in LUMO). There are three main organic semiconductors used in the experiments described in this thesis: Poly(2,5-bis(3-alkylthiophen-2-yl)thieno[3,2-b]thiophene (PBTTT), Poly(3-hexylthiophene-2,5-diyl) (P3HT) and Poly[[N,N9-bis(2-octyldodecyl)-naphthalene1,4,5,8-bis(dicarboximide)-2,6-diyl]-alt-5,59-(2,29-bithiophene)] (N2200). PBTTT is a p-type conjugated polymer with high field effect mobility up to $1 \mathrm{~cm}^{2} \mathrm{~V}^{-1} \mathrm{~s}^{-1}$. PBTTT was spin coated using a $10 \mathrm{mg} / \mathrm{ml}$ 1,4-dichlorobenzene (DCB) solution at $90{ }^{\circ} \mathrm{C}$. The resulting film was first dried at $120^{\circ} \mathrm{C}$ and then annealed at $180^{\circ} \mathrm{C}$ for 30 minutes. This provides a terrace morphology of PBTTT film with high degree of crystallinity as shown in Fig 3.1.

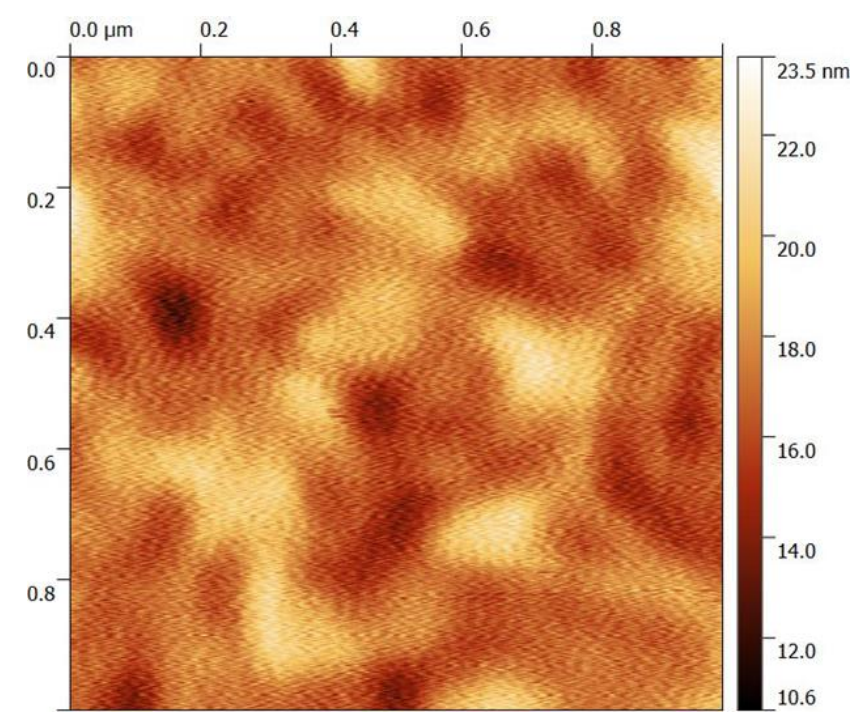

Figure 3.1 Surface morphology of PBTTT. 1 x $1 \mu \mathrm{m}$ AFM image of PBTTT (100 nm) deposited on Pt $(10 \mathrm{~nm})$. The RMS roughness is $1.539 \mathrm{~nm}$.

P3HT is a p-type organic semiconductor that has relatively short side chains compared to PBTTT. This in turn results in higher vertical mobility compared to PBTTT. P3HT was spin 
coated using a 10mg/ml 1,4-dichlorobenzene (DCB) solution. The resulting film was first dried at $100{ }^{\circ} \mathrm{C}$ and then annealed at $110{ }^{\circ} \mathrm{C}$ for 15 minutes. Both PBTTT and P3HT can be doped with p-type dopant, 2,3,5,6-Tetrafluoro-7,7,8,8-tetracyanoquinodimethane (F4TCNQ) via sequential doping method where films were immersed in $1.5 \mathrm{mg} / \mathrm{ml} \mathrm{F4TCNQ}$ acetonitrile solution. The doped samples were annealed at $80{ }^{\circ} \mathrm{C}$ for 20 minutes to achieve uniform distribution of dopant molecules.

In contrast, N2200 is a state of art n-type organic semiconductor with high field effect mobility approaching $1 \mathrm{~cm}^{2} \mathrm{~V}^{-1} \mathrm{~s}^{-1}$. N2200 was spin coated using a $10 \mathrm{mg} / \mathrm{ml}$ chlorobenzene (CB) solution at room temperature. The resulting film was first dried at $100^{\circ} \mathrm{C}$ and then annealed at $200{ }^{\circ} \mathrm{C}$ for 15 minutes. N2200 can be doped with n-type dopant Bis(cyclopentadienyl)cobalt(II) (CoCp2) by either sequential doping or mixing into the N2200 solution.
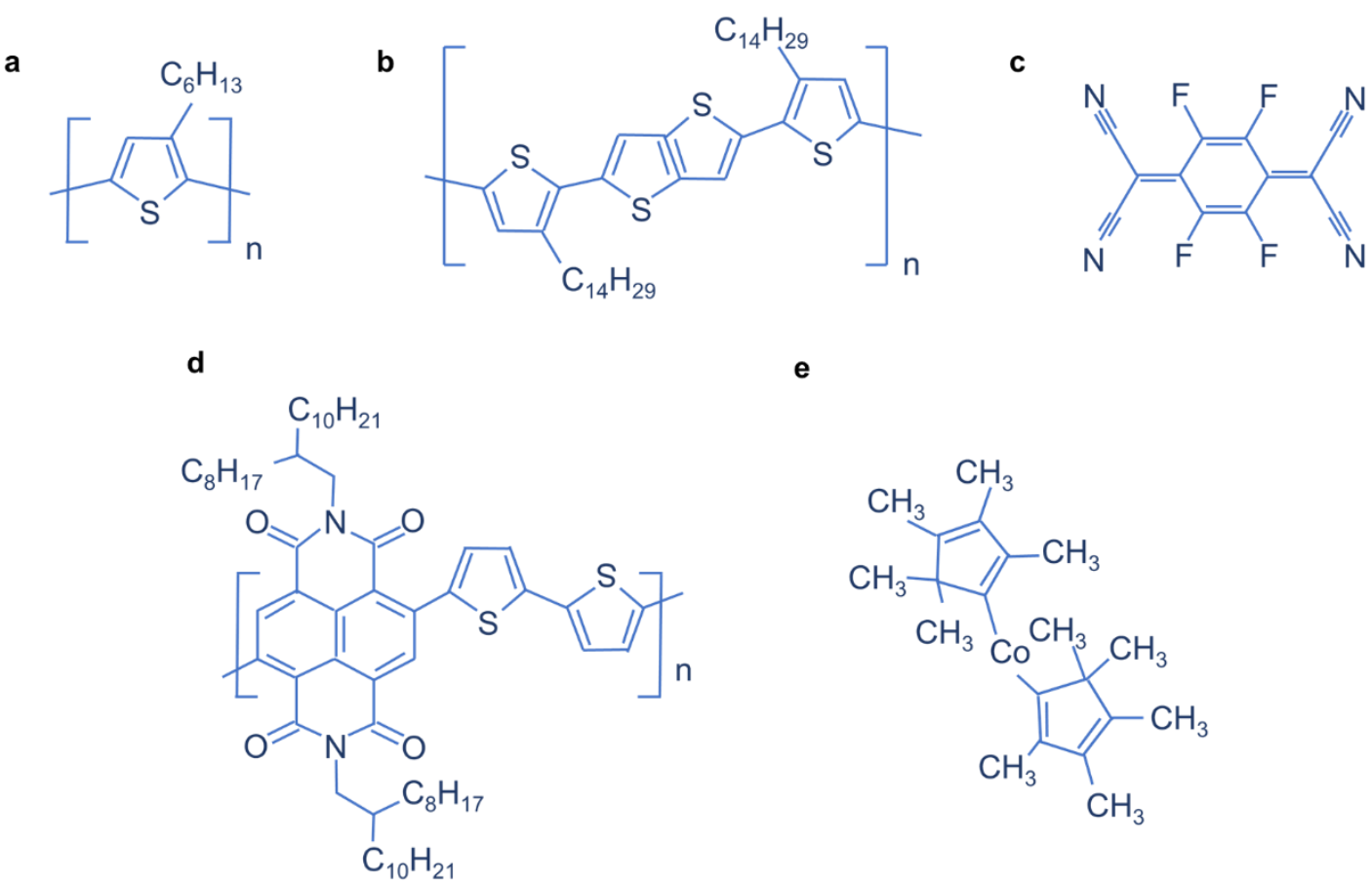

e

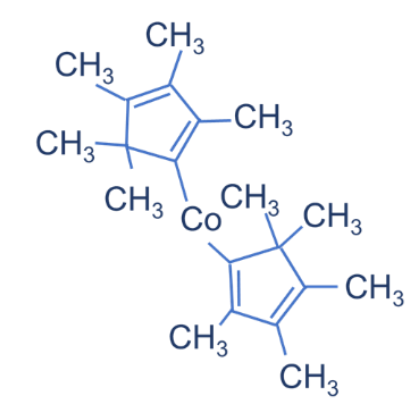

Figure 3.2 Molecular structures of main organic semiconductors studied. Molecular structures of P3HT (a), PBTTT (b), F4TCNQ (c), N2200 (d) and CoCp2 (e).

\subsection{Electrical spin injection}

\subsubsection{Vertical spin valves fabrication}

The large area devices were fabricated by evaporations through shadow masks and spin coating 
which formed a cross bar structure with $200 \times 200 \mu \mathrm{m}$ active junction size (Fig 4.6a, c). The thickness of the Py bottom electrode is $30 \mathrm{~nm}$ capped with $2 \mathrm{~nm}$ Au to prevent oxidation whereas the top Py electrode is $25 \mathrm{~nm}$. The coercive fields of the bottom and top Py electrodes are 0.1 and $6 \mathrm{mT}$, respectively. The difference in coercive field is created by different thickness and substrate surface.

\subsubsection{T Non-local spin valves fabrication}

Non-magnetic $\mathrm{Cr}(5 \mathrm{~nm}) / \mathrm{Au}(20 \mathrm{~nm})$ electrodes were defined on $1 \times 1 \mathrm{~cm} \mathrm{SiO} 2 / \mathrm{Si}$ substrate by standard optical lithography and deposited by thermal evaporation at a base pressure of $2 \times 10^{-7} \mathrm{mbar}$ and $1 \AA / \mathrm{s}$. Py $(25 \mathrm{~nm})$ electrodes were then defined by e-beam lithography and deposited by e-beam evaporation at a base pressure of $2 \times 10^{-7} \mathrm{mbar}$ and $2 \AA / \mathrm{s}$. The widths of the Py electrodes were 250 and $600 \mathrm{~nm}$ to ensure difference in coercive field. The $250 \mathrm{~nm}$ Py electrode has a coercive field of $14 \mathrm{mT}$ whereas the $600 \mathrm{~nm}$ Py electrode has a coercive field of $8 \mathrm{mT}$. The $\mathrm{AlO}_{\mathrm{x}}, \mathrm{MgO}$ and $\mathrm{Au}$ injection contacts were deposited onto Py in-situ without breaking the vacuum. PBTTT $(\sim 50 \mathrm{~nm})$ film was deposited and doped under the conditions as described in organic materials section. The doped PBTTT film was finally patterned into a dogbone structure by optical lithography and oxygen plasma etching (Fig 4.2a, c).

\subsubsection{Out of plane spin injection and in plane spin detection spin valves fabrication}

Bottom Py $(30 \mathrm{~nm})$ electrodes were defined on 1 x $1 \mathrm{~cm} \mathrm{SiO} / 2 / \mathrm{Si}$ substrate by e-beam lithography and deposited by e-beam evaporation at a base pressure of $2 \times 10^{-7} \mathrm{mbar}$ and $2 \AA / \mathrm{s}$. $\mathrm{Au}(2 \mathrm{~nm})$ injection contact was deposited on Py in-situ without breaking the vacuum by thermal evaporation at a base pressure of $2 \times 10^{-7} \mathrm{mbar}$ and $1 \AA / \mathrm{s}$ under $20 \mathrm{rpm}$ substrate stage rotation. The widths of the three bottom Py electrodes are 500, 2000 and $800 \mathrm{~nm}$, from left to right (Fig 4.4d). The coercive fields for the 500, 2000 and $800 \mathrm{~nm}$ electrodes are 8, 4 and $6 \mathrm{mT}$, respectively. PBTTT and P3HT (75 nm) were deposited under the conditions described previously. The top Py electrode was defined by optical lithography using an orthogonal resist that does not harm the organic semiconductor underneath. Py (30nm) was then deposited by thermal evaporation at a base pressure of $2 \times 10^{-7} \mathrm{mbar}$ and $0.5 \AA / \mathrm{s}$ (Fig 4.4a, e). The top Py electrode has a coercive field of around $7 \mathrm{mT}$. PBTTT $(\sim 50 \mathrm{~nm})$ film was deposited and doped with F4TCNQ under conditions described previously. 


\subsubsection{Electrical spin injection and inverse spin hall effect detection devices fabrication}

Non-magnetic $\mathrm{Cr}(5 \mathrm{~nm}) / \mathrm{Au}(20 \mathrm{~nm})$ electrodes were defined on 1 x $1 \mathrm{~cm} \mathrm{SiO} / \mathrm{Si}$ substrate by standard optical lithography and deposited by thermal evaporation at a base pressure of $2 \times 10^{-7} \mathrm{mbar}$ and $1 \AA / \mathrm{s}$. Pt (10 nm) electrode was defined by e-beam lithography and deposited by magnetron sputtering at a base pressure of $5 \times 10^{-7} \mathrm{mbar}$ and $5 \AA / \mathrm{s}$. Py $(60 \mathrm{~nm})$ electrode was defined next to the Pt electrode by e-beam lithography and deposited by e-beam evaporation at a base pressure of $2 \times 10^{-7} \mathrm{mbar}$ and $2 \AA / \mathrm{s}$. Au $(3 \mathrm{~nm})$ injection contact was deposited on Py in-situ without breaking the vacuum by thermal evaporation at a base pressure of $2 \times 10^{-7}$ mbar and $1 \AA / \mathrm{s}$ under $20 \mathrm{rpm}$ substrate stage rotation. PBTTT ( $\left.\sim 50 \mathrm{~nm}\right)$ film was deposited and doped with F4TCNQ under conditions described previously. The doped PBTTT film was finally patterned into a dog-bone structure by optical lithography and oxygen plasma etching (Fig 4.8a, b).

\subsubsection{Electrical spin injection measurements}

For DC measurements, current was applied by a KEITHLEY 2400 Source meter while voltage is measured by a KEITHLEY 2182A Nanovoltmeter. For AC measurements, standard lockin technique was used. All electrical spin injection measurements were performed in a Helium flow cryostat. The measurement configuration details for each device structure are given in results and discussion section.

\subsection{Dynamical spin injection}

\subsubsection{Tri-layer spin pumping devices fabrication}

Tri-layer spin pumping devices were prepared to study the vertical spin transport in organic semiconductors. Purified glass substrates were cut into $5 \times 3 \mathrm{~mm}$ dimensions to enable measurements using both ESR cavity and coplanar waveguide. A $10 \mathrm{~nm}$ Pt electrode was deposited on the glass substrate by magnetron sputtering at a base pressure of $5 \times 10^{-7} \mathrm{mbar}$ and $5 \AA /$ s. Organic conjugated polymers were then spin coated on the Pt electrode inside a Nitrogen glovebox. The thickness of the polymer films between 50 to $250 \mathrm{~nm}$ was controlled by varying the spin coating speed. For thicker polymer films, film thickness was controlled by drop casting with different solution concentrations. Py $(15 \mathrm{~nm})$ was thermally evaporated through a shadow mask with $1 \times 3 \mathrm{~mm}$ dimensions on top of the organic semiconductor at a 
base pressure of $2 \times 10^{-7} \mathrm{mbar}$ and $0.5 \AA / \mathrm{s}$. The Py stripe was capped with $5 \mathrm{~nm}$ Al to protect it from oxidation. The device was then patterned by oxygen plasma to remove the organic polymer outside the active area.

\subsubsection{Lateral spin pumping devices fabrication}

The devices were prepared using multiple step e-beam lithography and metal deposition on 5 x $3 \mathrm{~mm} \mathrm{SiO} / \mathrm{Si}$ substrates. Pt wire $(10 \mathrm{~nm})$ was deposited by magnetron sputtering at a base pressure of $5 \times 10^{-7} \mathrm{mbar}$ and $5 \AA / \mathrm{s}$. Py box $(25 \mathrm{~nm})$ was deposited by e-beam evaporation at a base pressure of $2 \times 10^{-7} \mathrm{mbar}$ and $2 \AA / \mathrm{s}$. The organic semiconductors were then spin coated from solution onto the devices. PBTTT and P3HT $(80 \mathrm{~nm})$ were deposited and doped with F4TCNQ under the conditions described previously. The organic semiconductor films were then patterned mechanically, and contact was made to the Pt wire using Ag paste. For PBTTT based LSP devices doped to $100 \mathrm{~S} / \mathrm{cm}$, the device resistance was $3.1 \mathrm{kOhm}$. When de-doped to $17 \mathrm{~S} / \mathrm{cm}$, the device resistance was $12.5 \mathrm{kOhm}$. For P3HT based LSP devices at $3.2 \mathrm{~S} / \mathrm{cm}$, the device resistance was $17.5 \mathrm{kOhm}$, at $0.3 \mathrm{~S} / \mathrm{cm}$ it changed to $25.3 \mathrm{kOhm}$.

\subsubsection{Spin pumping measurements}

Ferromagnetic resonance (FMR) of Py was excited in both an electron spin resonance (ESR) system and a coplanar waveguide setup. For measurements in ESR setup (Bruker E500 Xband spectrometer), the sample was placed in the centre of a Bruker ER 4122SHQE cavity at a microwave frequency of $9.38 \mathrm{GHz}$. Two ends of the Pt wire were connected to a KEITHLEY 2182A Nanovoltmeter with Ag paste attached $\mathrm{Cu}$ wires to detect the voltage of the device. For measurements with coplanar waveguide, the sample is flipped onto the coplanar waveguide where a layer of polyamide isolated the sample from the waveguide. Both ends of the Pt wire are connected to a KEITHLEY 2182A Nanovoltmeter by Ag paste. Low temperature spin pumping measurements were performed by positioning a coplanar waveguide into a Helium flow cryostat with microwave access. 


\section{Chapter 4}

\section{Electrical spin injection into organic semiconductors}

\subsection{Motivation}

The observation of GMR in $\mathrm{Alq}_{3}$ based vertical spin valve in 2004 triggered intense research into spin transport in a variety of organic semiconductors using two terminal spin valve approach. However, much scepticism arises in recent years as to whether this measurement approach is an adequate method to probe spin transport in organic semiconductors since such magnetoresistance response may originate from pin-holes, tunnelling through hot spots and tunnelling anisotropic magnetoresistance associated with LSMO ferromagnetic contacts [39], [131], [132], [74], [40]. Therefore, it is necessary to find an artefact-free technique to probe spin transport in organic semiconductors. Standard four terminal non-local spin valve is the most widely accepted method to study spin dynamics in inorganic semiconductors and metals. This chapter begins with optimising the charge injection properties at the ferromagnet and organic semiconductor interface to maximise magnetoresistance in lateral spin valve devices using established theoretical work, follows by a systematic study of non-local organic spin valve devices based on doped high mobility organic semiconductors. Evolution from conventional non-local organic spin valve device to novel out of plane spin injection and in plane spin detection and electrical spin injection and ISHE detection devices will be discussed. The fundamental challenge of non-local spin injection and detection in organic semiconductors is identified through this exploratory work. In addition, the effect of shrinking junction size of vertical organic spin valve based on $3 \mathrm{~d}$ metallic ferromagnetic electrodes without tunnelling barrier is investigated.

Note: This project was conducted jointly with Dr. Deepak Venkateshvaran and Dr. Murat Cubukcu. The $\mathrm{Co} / \mathrm{Al}_{2} \mathrm{O}_{3}$ film was deposited in SPINTEC by Dr. Laurent Vila and Dr. Stephane Auffret. 


\subsection{Electrical spin injection and detection experiments}

\subsubsection{Charge injection properties across ferromagnet/organic semiconductor interfaces}

Fig 4.1a shows a simulated magnetoresistance for doped PBTTT under different resistance area product. The simulation was completed using parameters for doped PBTTT [the carrier concentration, $n=10^{20} \mathrm{~cm}^{-3}$ (estimated using Hall effect and ESR measurement), the carrier mobility, $\mu=1 \mathrm{~cm}^{2} / \mathrm{Vs}$, the conductivity, $\sigma=100 \mathrm{~S} / \mathrm{cm}$, spin lifetime, $\tau_{\mathrm{s}}=100 \mathrm{~ns}$ (estimated using ESR measurement), the spin diffusion length, $\lambda_{\mathrm{s}}=500 \mathrm{~nm}$ (estimated using Einstein relation with optimistic parameters) and the distance between ferromagnetic electrodes, $L_{\mathrm{FM}}=$ $100 \mathrm{~nm}]$ in the model developed by Fert et al as described in Chapter 2.1.1.

Fig $4.1 \mathrm{~b}$ shows the bias dependent resistance area product for spin injection into doped PBTTT from a variety of contacts. The injection properties have been optimised such that the resistance area product falls within the range where magnetoresistance is expected to be maximised. Here, the $\mathrm{MgO}$ tunnel barriers were prepared in-situ following the Py evaporation by either magnetron sputtering or e-beam evaporation. The $\mathrm{Al}_{2} \mathrm{O}_{3}$ tunnel barrier was prepared in-situ following the Py evaporation by magnetron sputtering of aluminium and followed by exposure to oxygen plasma without breaking the vacuum. Note, non-local magnetoresistance and/or tunnel magnetoresistance can be observed in metal and graphene-based spin valves using the $\mathrm{MgO}$ and $\mathrm{Al}_{2} \mathrm{O}_{3}$ tunnel barriers prepared which indicates the quality of the tunnel barriers is high. The Au capping layer is thermally evaporated onto Py electrodes without breaking vacuum under continuous sample stage rotation to improve the uniformity of the film. The bias dependence of the resistance area product originates from the non-linear current voltage relationship of the doped PBTTT for the Py injector capped with $3 \mathrm{~nm} \mathrm{Au}$. Whereas, the bias dependence of the resistance area product for Py injectors capped with oxide barriers comes from both the doped PBTTT and the oxide barrier (combined effect) and hence resulted a stronger dependence on the bias applied compared to devices with Py injectors capped with $3 \mathrm{~nm} \mathrm{Au}$. 
a

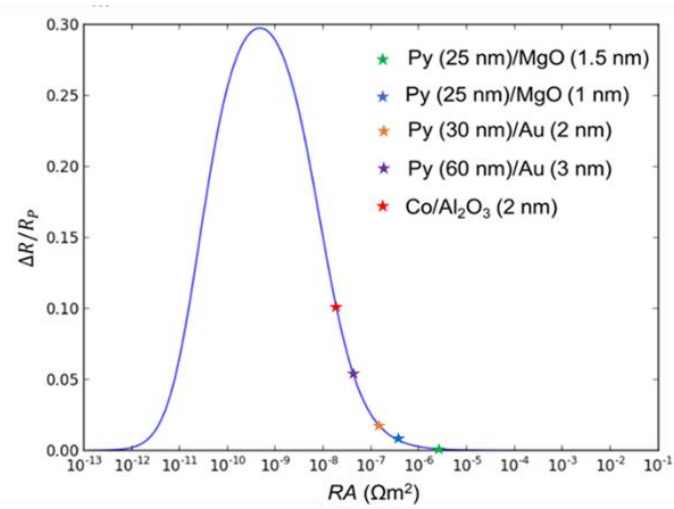

b

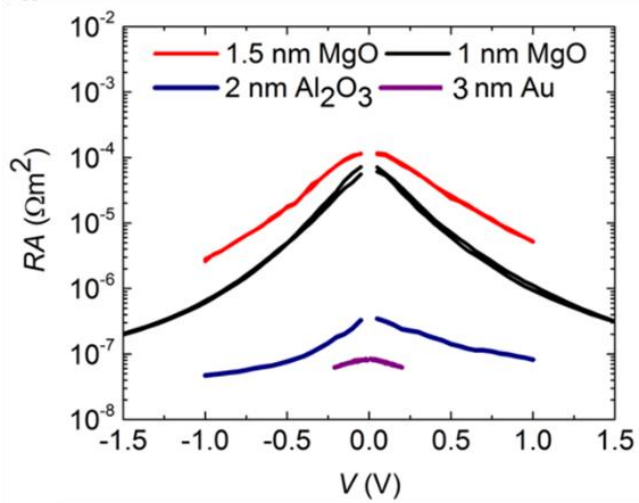

Figure 4.1 Electrical spin injection interface properties. a, Simulated magnetoresistance for a typical organic semiconductor vertical spin valve as a function of Resistance-Area product under the assumptions of Boltzmann transport theory. The star symbols locate the experimental Resistance-Area product values on the plot. b, Experimental Resistance-Area product of various injection contacts as a function of applied bias.

\subsubsection{Non-local spin valves}

An unambiguous solid state device-based approach to measure spin diffusion in inorganic semiconductors is to use nonlocal lateral architectures where spin diffusion is decoupled from spin drift within the same device. Pure diffusive spin transport is then probed by measuring the spin accumulation above a ferromagnetic detector electrode in relation to that at the ferromagnetic injector. Such nonlocal spin valve measurements have successfully quantified spin diffusion lengths in inorganic materials such as graphene, Ge, GaAs and various metals [48], [49], [50], [51], [52], [53], [76].

Fig 4.2a shows a schematic illustration of an organic non-local spin valve device that consists of two permalloy electrodes in close proximity with each other situated in between two nonmagnetic gold electrodes where patterned organic semiconductor bridged on top of all electrodes. The measurement involves sending a current from one ferromagnetic electrode to a non-magnetic electrode ( $\mathrm{Au} \mathrm{pad}$ ) and probing the voltage between the other ferromagnetic electrode and non-magnetic electrode while sweeping the magnetic field applied in plane. The drift spin polarised current follows the current direction while diffusive pure spin current diffuse to the adjacent ferromagnetic electrode. This results in a change in resistance dependent on the relative magnetisation alignment of the ferromagnetic electrodes (Fig 4.2b). Fig 4.2c 
shows an optical micrograph of the completed device where the organic semiconductor is patterned into a "dog-bone" structure (dark blue) using optical lithography. The organic semiconductor is $10 \mu \mathrm{m}$ wide in the narrowest segment that overlaps the ferromagnets. With the patterning process for organic semiconductors used here, one is limited to minimum feature sizes of $3 \mu \mathrm{m}$ [133]. The gap between Py electrodes is well-resolved down to around $100 \mathrm{~nm}$ as confirmed by SEM image (Fig 4.2d).

The two ferromagnetic electrodes need to have different coercive fields to achieve the antiparallel magnetisation state. The difference in coercive fields can be obtained by using different widths of Py electrodes due to magnetic anisotropy. The coercive field can be measured using anisotropic magnetoresistance measurement where external magnetic field is sweep along the Py electrode when a current is applied along the Py electrode (Fig 4.2e). The $600 \mathrm{~nm}$ wide Py electrode has a coercive field of $8 \mathrm{mT}$ while the $250 \mathrm{~nm}$ narrow Py electrode has a coercive field of $14 \mathrm{mT}$. Note, the $600 \mathrm{~nm}$ wide Co electrode has a coercive field of 20 $\mathrm{mT}$ and the $250 \mathrm{~nm}$ narrow Co electrode has a coercive field of $40 \mathrm{mT}$. Fig $4.2 \mathrm{f}$ shows the current-voltage measurement of doped PBTTT across the Au pads at room temperature, this provides an estimate of conductivity of around $50 \mathrm{~S} / \mathrm{cm}$. Even though the current-voltage characteristics for doped PBTTT looks linear, but the slight curvature in differential conductance characteristics indicate the slight non-linearity in the current-voltage relationship.

Fig 4.3a shows a typical field dependence of the non-local voltage with $10 \mu \mathrm{A}$ injection current for a doped PBTTT based lateral spin valve device with $1 \mathrm{~nm} \mathrm{MgO}$ tunnel barrier deposited on top of Py to enhance spin injection [134]. The noise level is hundreds of $\mu \mathrm{V}$ when the measurement is performed in DC (representing around $20 \%$ the value of the offset). Furthermore, non-local organic spin valve with $2 \mathrm{~nm}$ aluminium oxide $\left(\mathrm{AlO}_{\mathrm{x}}\right)$ tunnel barrier was attempted since $\mathrm{AlO}_{\mathrm{x}}$ is known to be more stable in ambient environment. However, the noise fluctuation in this case only shows a slight improvement over the $\mathrm{MgO}$ barrier case. The noise level can be reduced to a few $\mu \mathrm{V}$ when $\mathrm{MgO}$ tunnel barrier is replaced with a thin gold capping layer (representing around 5-10\% the value of the offset) (Fig 4.3c). Thin gold capping layer is used to prevent Py from oxidation in air which is known to supress spin injection properties [76]. Large noise levels in the case of devices with $\mathrm{MgO}$ tunnel barriers are believed to be caused by moisture absorption upon exposure to ambient conditions before the deposition of an organic semiconductor film, and/or an un-optimum packing of the organic semiconductor over Py/MgO electrodes. These two effects lead to charge carrier scattering and limited carrier 
injection at the interface [135].

It is of essence to note that the necessity of having a tunnel barrier such as $\mathrm{MgO}$ or $\mathrm{Al}_{2} \mathrm{O}_{3}$ in organic spin valves to overcome conductance mismatch at the interface between the organic and the ferromagnet is currently under debate. The charge injection in organic semiconductors is often mediated by variable range hopping which tunnelling transport is involved. Therefore, a natural tunnelling barrier is formed between the metallic ferromagnet and organic semiconductor interface that overcomes the inherent conductivity mismatch problem [72], [136]. In addition, from the resistance area product analysis, it is shown that the resistance area product for $3 \mathrm{~nm}$ gold capped Py is within the range to give a strong magnetoresistance signal. Thus, $3 \mathrm{~nm}$ gold capped Py is used as the spin injector for spin valve devices. By doing an AC lock-in measurement, the noise level can be further reduced to a few hundred $\mathrm{nV}$ in the case of gold capped device (representing under $1 \%$ the value of the offset) (Fig 4.3d). However, no non-local spin signal can be measured.

In addition, the junction area over which spin polarised carriers are injected into the active layer of the non-local spin valve from a ferromagnet is known to affect the strength of the measured spin signal [137]. For this reason, the junction area for injection in typical non-local spin valves is around a few hundred square nanometers, much smaller than the square of the spin diffusion length of the active layer. The patterning process of the organic semiconductor has its limitations in achieving such small junction areas to ensure that the square of the spin diffusion length is far larger than the injection area. This poses an additional roadblock in the successful working of organic non-local spin valves and is among the many reasons that make this experiment challenging.

To summarise, a nonlocal spin valve signal has never been successfully measured despite multiple diligent attempts in conjugated organic semiconductors [138], [139]. This is primarily because the noise fluctuations on the measured nonlocal voltage are induced by the fundamental nature of stochastic hopping transport in these disordered materials (comprehensive study in Chapter 4.2.5) [54] and outsizes the spin accumulation on the detector electrode rendering such a measurement extremely challenging if not impossible. Spin injection into doped organic semiconductors using other novel device architectures and measurement techniques shown in Chapter 4.2.3 and 4.2.5 were attempted following the failure of conventional nonlocal spin valves at successfully probing spin transport in organic 
semiconductors.

a

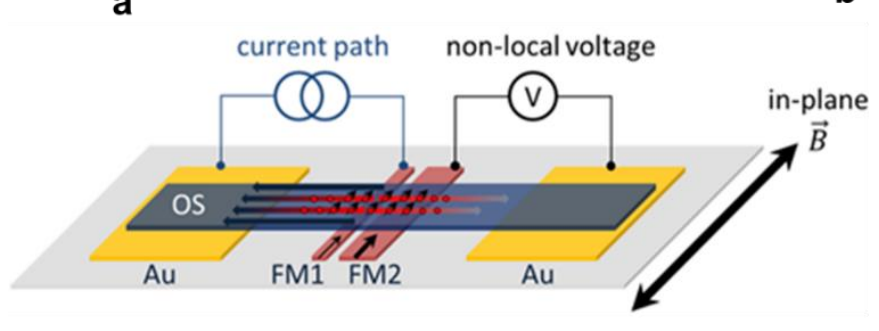

C

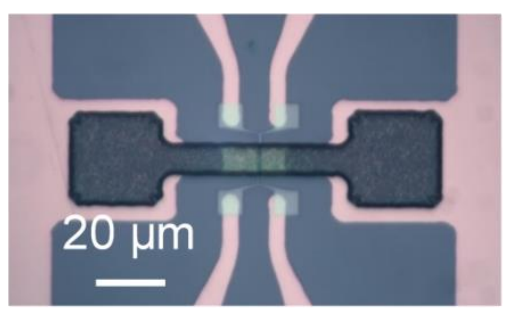

e

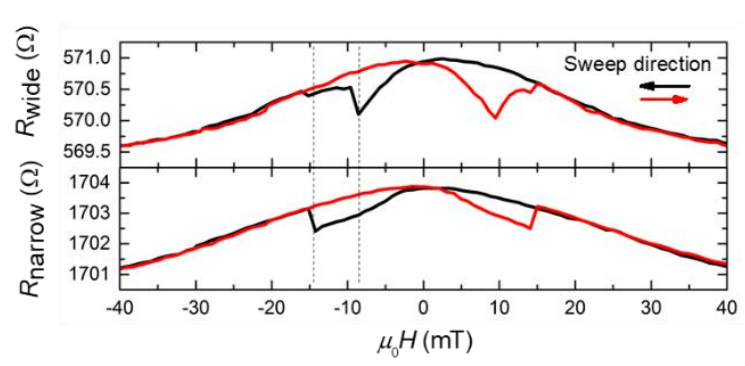

b

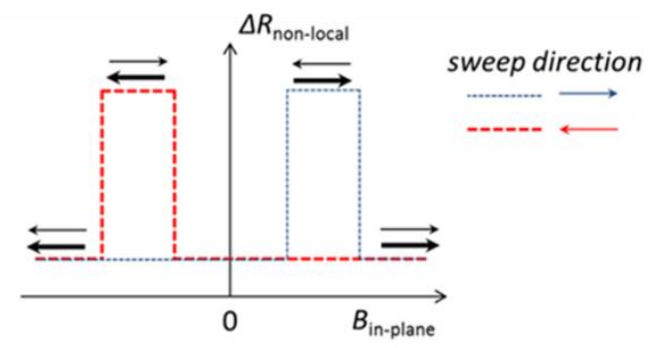

d

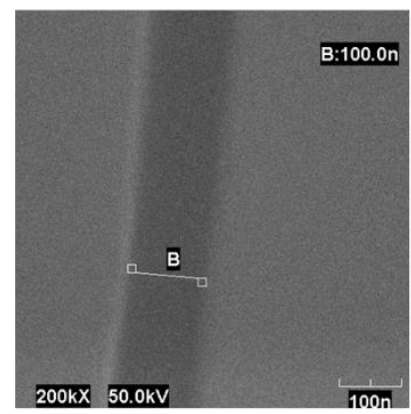

f

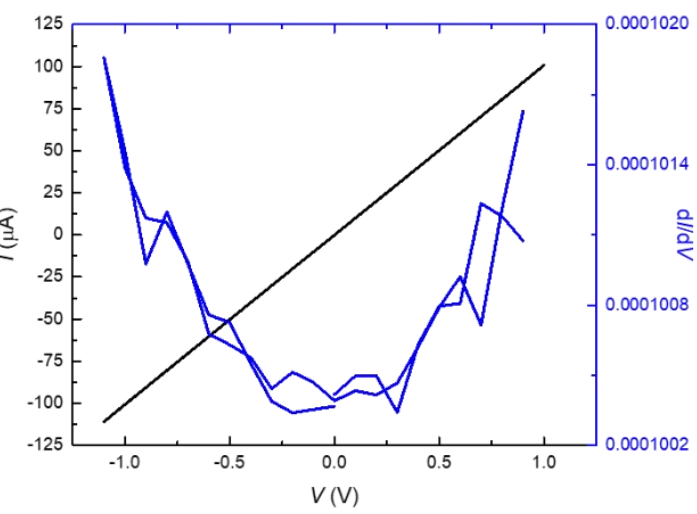

Figure 4.2 Non-local spin valves based on organic semiconductors. a, Schematic non-local spin valve measurement configuration. b, Expected measurement outcome for non-local voltage as a function of applied magnetic field. The black arrows indicate the relative magnetisation orientation of the Py electrodes. c, Optical image of a typical non-local spin valve device. The dark blue 'dog-bone' structure is the patterned organic semiconductor. d, SEM image of the gap between Py electrodes. The scale bar is $100 \mathrm{~nm}$. e, Anisotropic magnetoresistance (AMR) of $600 \mathrm{~nm}$ wide (top) and $250 \mathrm{~nm}$ narrow (bottom) Py electrodes. The dashed black lines indicate the coercive field for wide ( $8 \mathrm{mT})$ and narrow (14 mT) Py electrodes. The solid black and red lines represent the forward and reverse field sweep direction, respectively. f, $I-V$ characteristics across the Au pads at $300 \mathrm{~K}$ (black line). The blue line is the differential conductance of the black line. The organic semiconductor is F4TCNQ doped PBTTT with a conductivity of around $50 \mathrm{~S} / \mathrm{cm}$. 
a

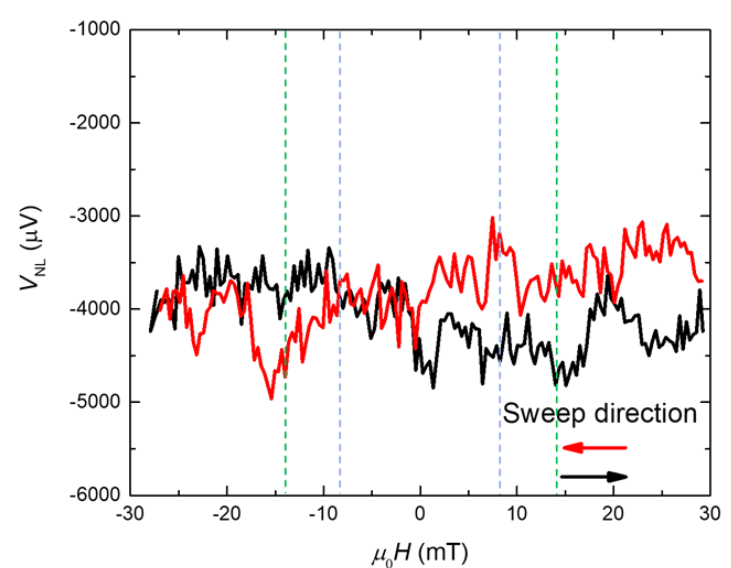

C

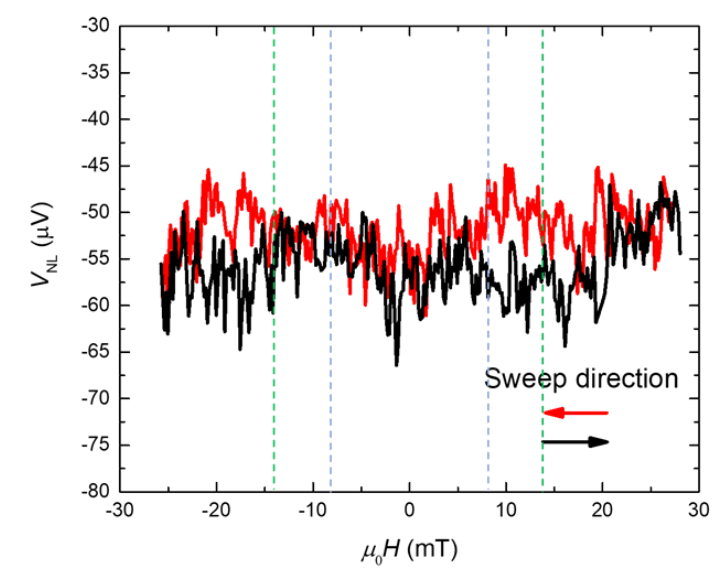

b

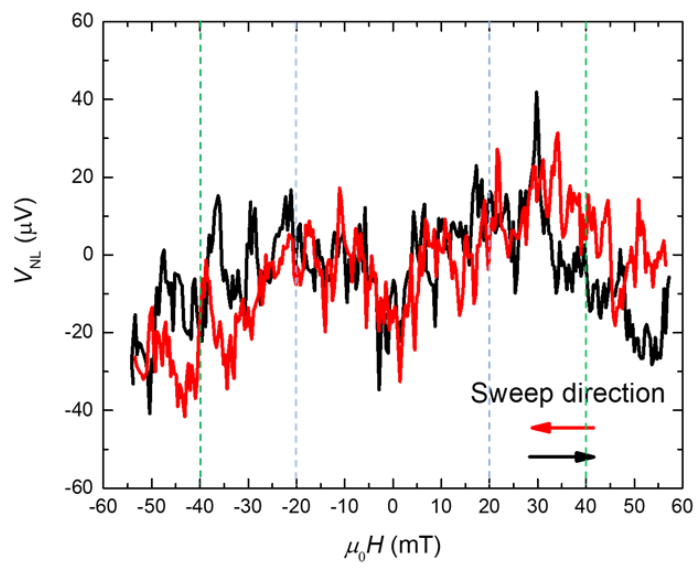

d

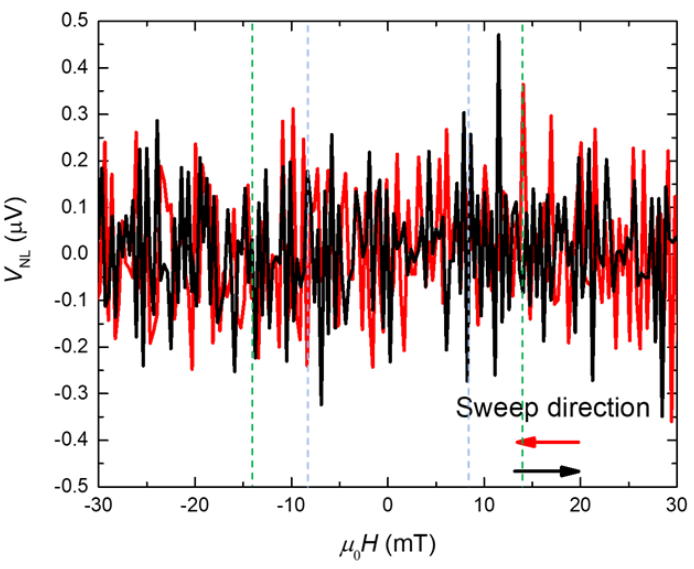

Figure 4.3 Organic non-local spin valves measurements. a, DC field dependence of the non-local voltage, $V_{\mathrm{NL}}$ for device with $1 \mathrm{~nm} \mathrm{MgO}$ tunnel barrier on top of Py. The injected current is $10 \mu \mathrm{A}$ from narrow FM electrode to NM Au pad. b, Lock-in field dependence of non-local voltage for device with $2 \mathrm{~nm} \mathrm{AlO}_{\mathrm{x}}$ tunnel barrier on top of Co. The lock-in frequency is set at $79 \mathrm{~Hz}$ and the AC voltage applied is $0.1 \mathrm{~V}$. $\mathbf{c}$, DC field dependence of the non-local voltage, $V_{\mathrm{NL}}$ for device with $3 \mathrm{~nm}$ Au capping layer on top of Py. The injected current is $10 \mu \mathrm{A}$ from narrow FM electrode to NM Au pad. d, Lock-in field dependence of non-local voltage for device with $3 \mathrm{~nm}$ Au capping layer on top of Py. The lock-in frequency is set at $79 \mathrm{~Hz}$ and the $\mathrm{AC}$ voltage applied is $0.2 \mathrm{~V}$. The green and blue dashed lines indicate the coercive fields for narrow and broad FM electrodes, respectively. The measurement temperature is $300 \mathrm{~K}$. 


\subsubsection{Out of plane spin injection and in plane spin detection spin valves}

The out of plane spin injection non-local spin valve aimed at increasing the spin accumulation by increasing current density in the device that can be achieved by vertical spin injection. This is achieved by increasing the electric field across a film thickness of circa 50 nanometers (rather than laterally across a film of a few micrometres as in the case of non-local spin valves). The vertical drift current from the central ferromagnetic electrode to the top electrode created a lateral diffusive pure spin current as such the non-local voltage probed by the outer electrodes will depend on the relative magnetisation of the bottom electrodes as illustrated in Fig 4.4a, b and c. This symmetric architecture automatically reduces the offset voltage on the nonlocal arm. The top permalloy electrode enables this device architecture to simultaneously measure vertical spin transport in the organic semiconductor via vertical spin valve measurements.

Fig 4.4d shows a typical SEM image of the bottom Py electrodes with well-resolved gap between electrodes. Patterning top Py electrode on top of organic semiconductors is challenging as conjugated organic polymers can be damaged easily by exposure to chemical solvents such as the developer solution for optical lithography or solvent solution for lift-off process. As a result, a special processing procedure is developed using orthogonal resist that does not harm the organic polymers. The organic semiconductor film is first coated with this resist and another layer of S1813 resist is then spin coated on top of the orthogonal resist. The bilayer resist is then patterned using optical lithography and then developed in developer solution for S1813 where the orthogonal resist protects the organic semiconductor from exposure to S1813 developer solution. The structure is then placed in the developer solution for the orthogonal resist which does harm the organic semiconductors. This creates the desired pattern on top of the organic semiconductor with an "undercut" which makes lift-off process straightforward as illustrated in Fig 4.4e. The device structure is then placed in the solvent solution for lift-off after Py evaporation, note this solvent solution for lift-off is orthogonal to the organic semiconductors. The final completed device is illustrated in Fig 4.4f.

The process of Py metal layer lift-off in orthogonal solvent leads to de-doping of F4TCNQ dopants from PBTTT. Nevertheless, the current scales accordingly with the junction area which indicates defect-free device has been fabricated (Fig 4.5a, b). The completed device can be doped by immersing it in solution of F4TCNQ and acetonitrile (Fig 4.5c). Fig 4.5d shows the 
field dependence of the voltage between left and right FM electrodes when a $1 \mu \mathrm{A}$ current is injected from the middle FM electrode to the top electrode. Once again, relatively high noise level around few hundred $\mu \mathrm{V}$ is observed. Even though AC lock-in measurement reduces the noise level up to a factor of ten, no spin signal can be probed in this configuration. The AC lock-in measurement technique reduces measurement noise primarily by limiting the bandwidth which reduces Johnson noise. In addition, the measurement frequency also affects the $1 /$ f flicker noise generated via hopping conduction in organic semiconductors (please refer to Chapter 4.2.5 for more in-depth discussion). This said, such an out-of-plane spin drift with in-plane diffusion device architecture is a new concept device that could be implemented with other organic or inorganic materials in the future. 
a

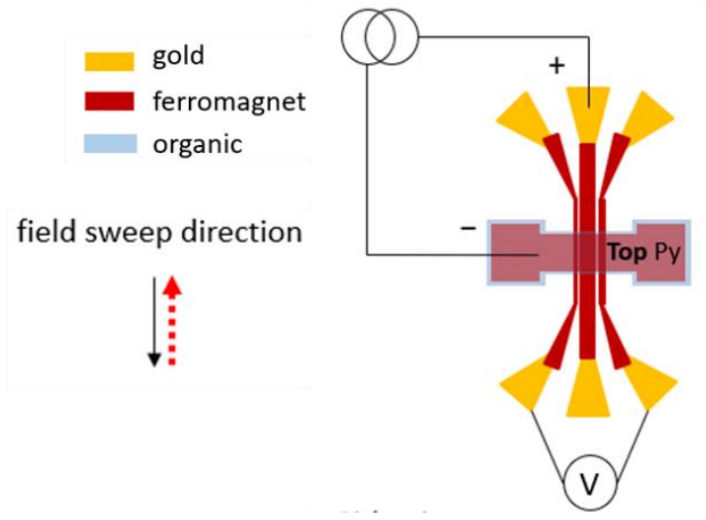

C

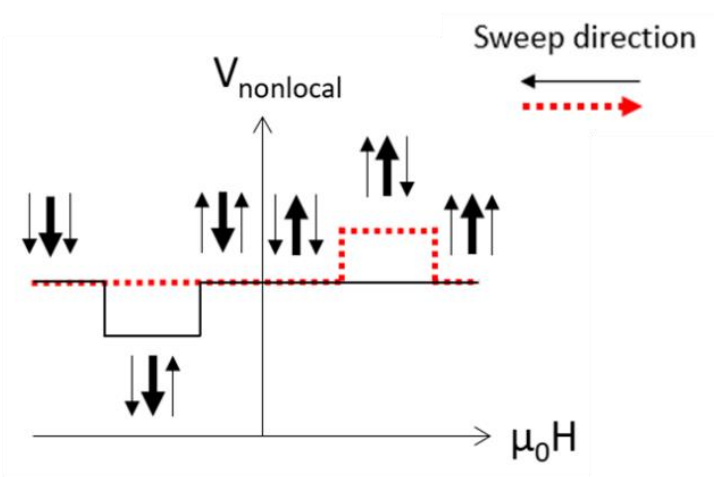

d

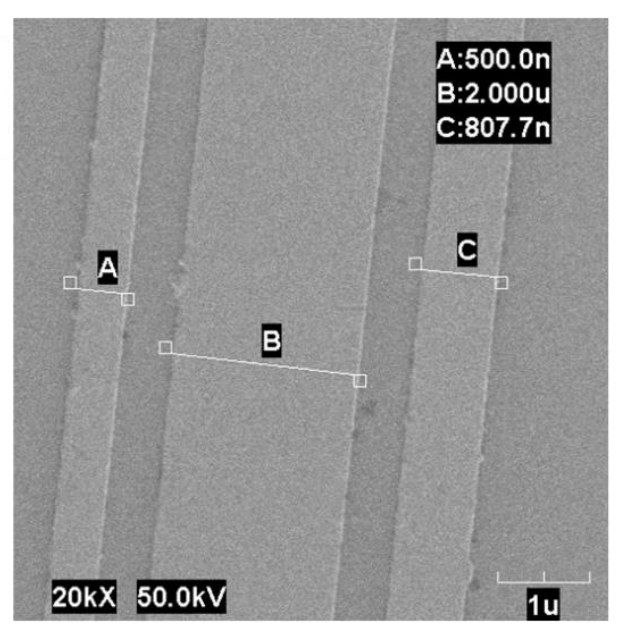

e

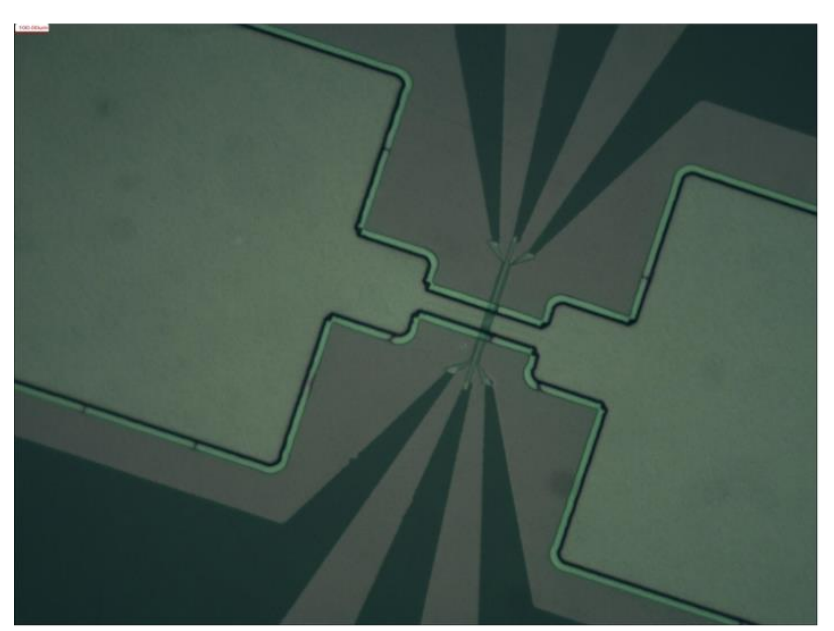

$\mathbf{f}$

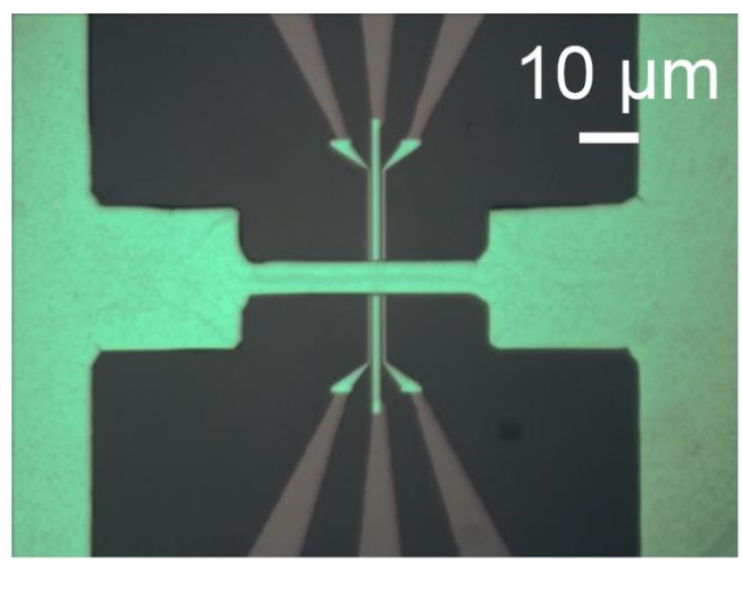

Figure 4.4 Two in one organic spin valve device. a, Schematic out of plane spin injection and in plane spin detection measurement configuration. $\mathbf{b}$, Side view of the measurement configuration. $\mathbf{c}$, Expected measurement outcome for the non-local voltage between the two outer Py electrodes as a function of applied magnetic field. The black arrows indicate the magnetisation direction of the bottom three Py electrodes. d, SEM image of the bottom three Py electrodes. e, Optical image of the top Py electrode pattern on top of organic semiconductor before Py evaporation. f, Optical image of a typical two in one 
organic spin valve device.

a

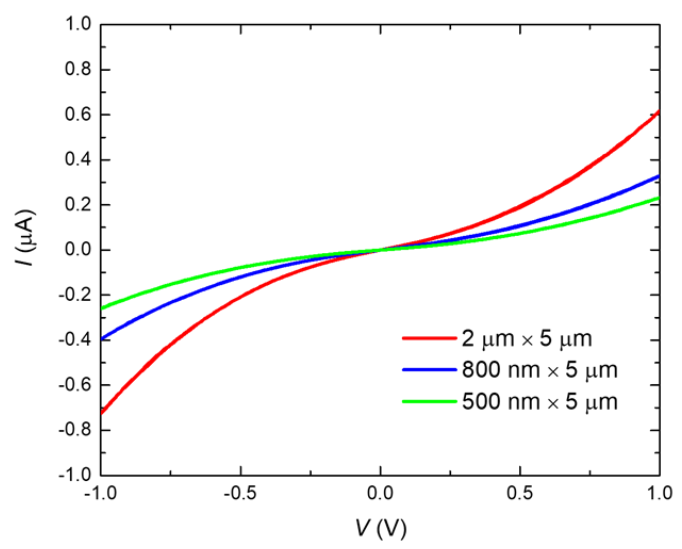

C

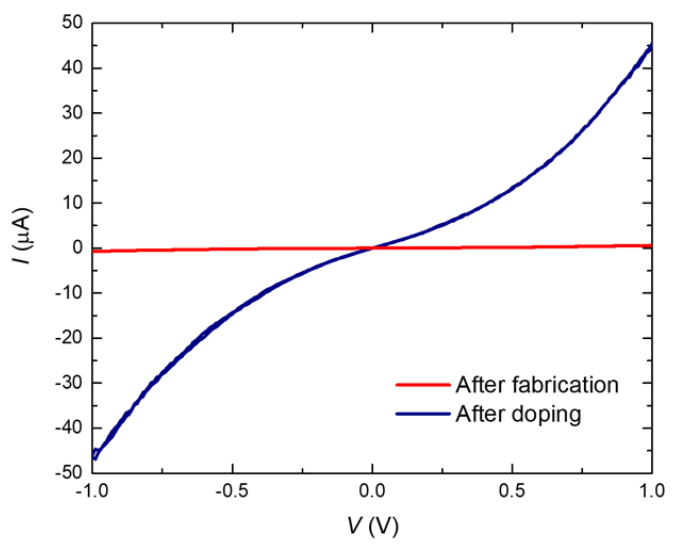

b

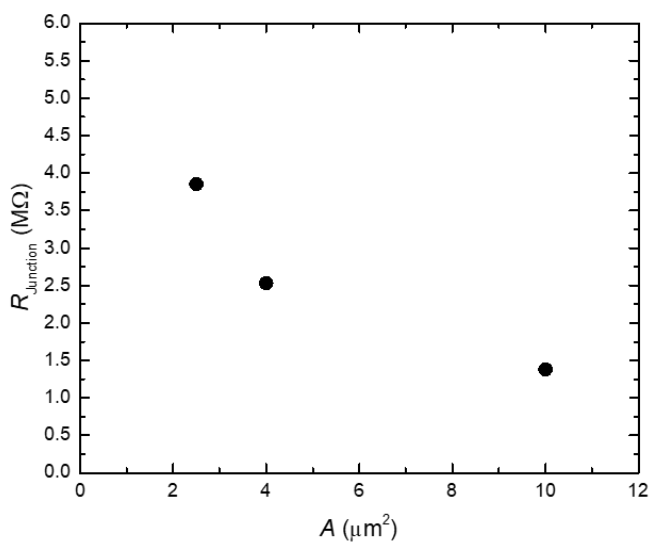

d

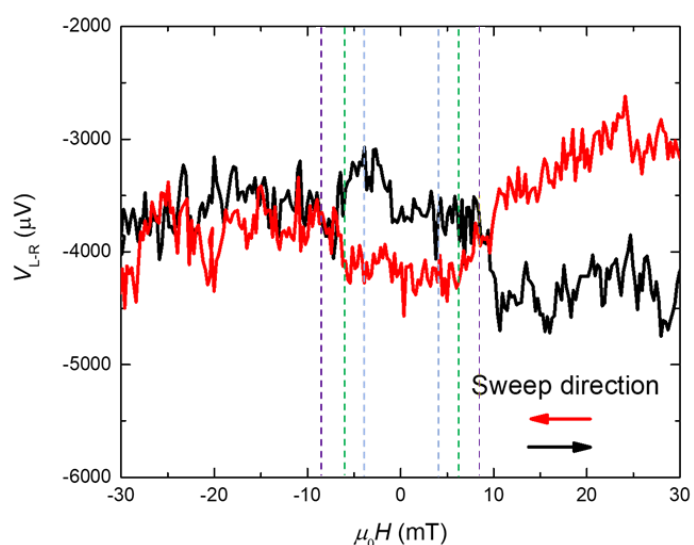

Figure 4.5 Out of plane spin injection and in plane spin detection measurement. a, $I-V$ characteristics of the device with different junction areas (current applied across the top electrode and bottom electrodes with different widths). $\mathbf{b}$, Resistance of the junction, $R_{\text {Junction }}$ as a function of junction area, $A$. The voltage applied is $1 \mathrm{~V}$. c, $I-V$ characteristics of the $2 \mu \mathrm{m}$ x $5 \mu \mathrm{m}$ junction directly after fabrication and after doping with F4TCNQ in acetonitrile. d, Field dependence of the voltage across the left and right FM electrodes, $V_{\mathrm{L}-\mathrm{R}}$. The current injected from the middle FM electrode to top electrode is $1 \mu \mathrm{A}$. The blue, green and purple dashed lines indicate the coercive fields for middle, right and left bottom FM electrodes, respectively. The measurement temperature is $300 \mathrm{~K}$. 


\subsubsection{Vertical spin valves}

Until now, the field of organic spintronics has mainly used two terminal vertical spin valves to understand spin transport in organic materials. Most of the work used manganite LSMO as the spin injector. This material however has both in plane and out of plane magnetisation which could generate a tunnelling anisotropic magnetoresistance (TAMR) signal if it is used as a ferromagnetic electrode in vertical spin valve devices. In addition, the formation of pinholes in organic spin valve with tunnelling barrier could give rise to magnetoresistance originating from simple tunnel magnetoresistance (Top Ferromagnet/Tunnel barrier/Bottom Ferromagnet).

In this work, we have attempted to understand spin injection in doped PBTTT by studying vertical spin valve structures with $3 \mathrm{~d}$ metallic ferromagnetic electrodes in the absence of tunnel barrier. Figure below shows the device structure and measurement configuration along with expected measurement outcome. The large area devices were fabricated by evaporations through shadow masks and spin coating which formed a cross bar structure with $200 \times 200 \mu \mathrm{m}$ active junction size (Fig 4.6a, c). The thickness of the Py bottom electrode is $30 \mathrm{~nm}$ capped with $2 \mathrm{~nm} \mathrm{Au}$ to prevent oxidation whereas the top Py electrode is $25 \mathrm{~nm}$. The coercive fields of the bottom and top Py electrodes are 0.1 and $6 \mathrm{mT}$, respectively. The difference in coercive field is created by different thickness and substrate surface. The spin valve measurements were performed by quasi-four points method with in plane applied magnetic field. Depending on the relative alignment of magnetisation between top and bottom electrode, one should observe a difference in resistance due to spin dependent scattering in the organic spin transport layer (Fig 4.6b).

Fig 4.6d, e show a comparison between a shorted spin valve and a non-shorted spin valve distinguished by a current spreading control experiment. This measurement involves sending a current through either the top or bottom electrode and probing the voltage of the other electrode. In the case of shorted device, clear anisotropic magnetoresistance (AMR) signal was observed in both electrodes while AMR signal can only be observed in the electrode with current flowing through for non-shorted device. An attempt has been made to measure spin valve response of the shorted device. However, only AMR signal can be observed in both local and quasi-four points measurements (Fig 4.6f). This is consistent with other published work that ferromagnetic electrodes in direct contact does not generate a spin valve signal [140], [141]. 
a

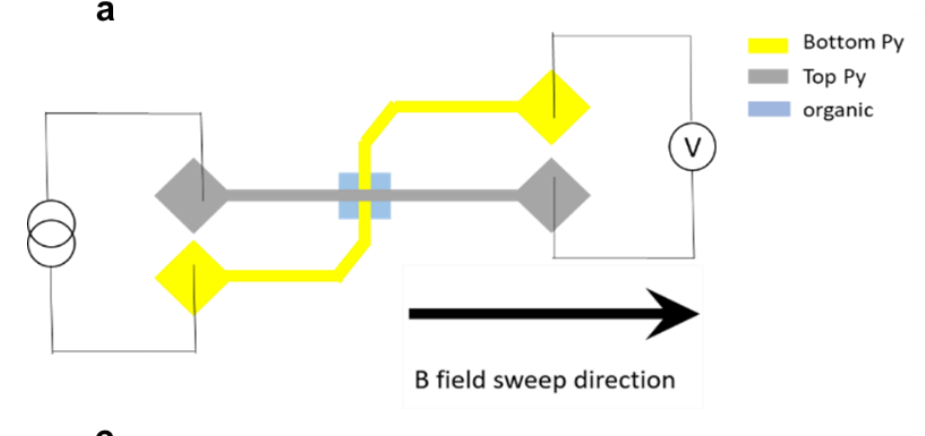

C

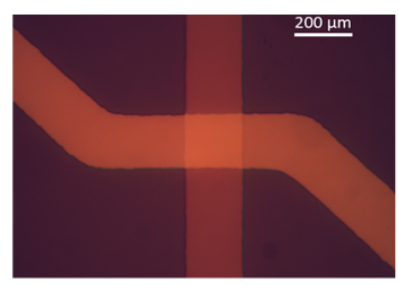

e

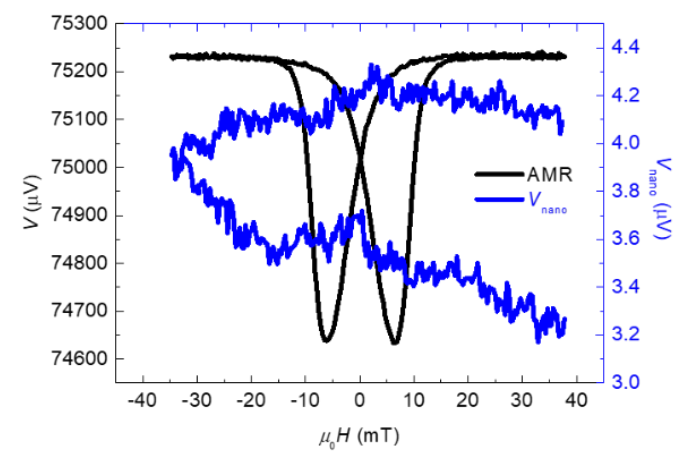

b

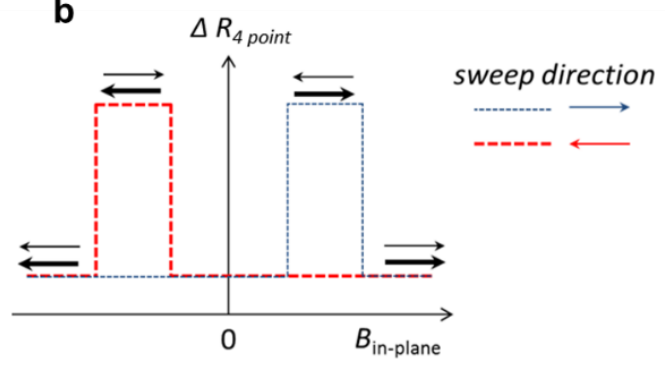

d

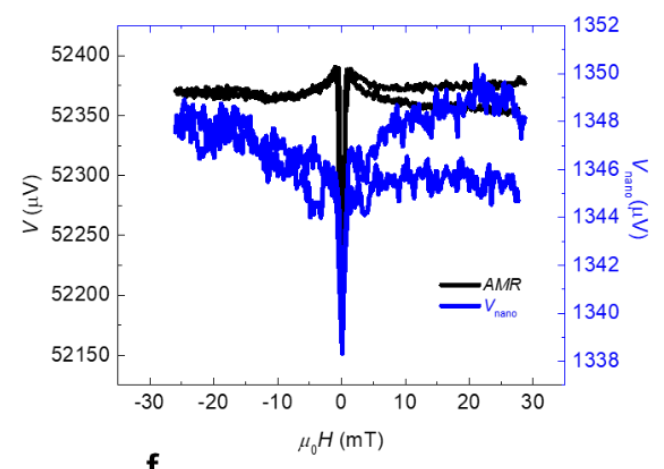

f

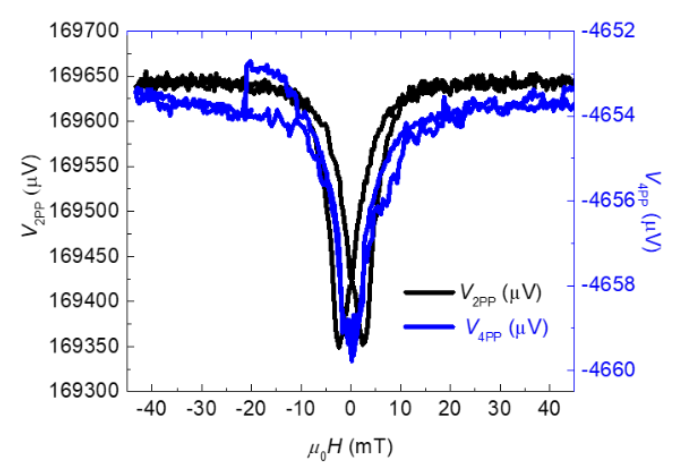

Figure 4.6 Large area $(200 \times 200 \mu \mathrm{m})$ vertical organic spin valve device. a, Schematic vertical spin valve measurement configuration. $\mathbf{b}$, Expected measurement outcome for the quasi-four points device resistance as a function of applied magnetic field. The black arrows indicate the relative magnetisation direction between the top and bottom Py electrodes. c, Optical image of the vertical spin valve device. The organic semiconductor is patterned under the top Py electrode by oxygen plasma. d-e, Current spreading control experiment for both a shorted (d) and a non-shorted (e) vertical organic spin valve device. A $100 \mu \mathrm{A}$ current was send through one Py electrode while probing the voltage of the corresponding Py electrode. f, Spin valve measurement for the shorted device. The injected current is $100 \mu \mathrm{A}$ and the voltage is measured using two points, $V_{2 \mathrm{pp}}$ and quasi-four points, $V_{4 \mathrm{pp}}$ geometry. The measurement temperature is $90 \mathrm{~K}$ and the thickness of F4TCNQ-PBTTT is $75 \mathrm{~nm}$. 
Fig $4.7 \mathrm{a}$ shows a magnetoresistance measurement for the non-shorted device at room temperature and $90 \mathrm{~K}$, a clear spin valve signal that depends on the relative alignment of ferromagnetic electrode magnetisation can be observed. By capping the bottom Py electrode 2 $\mathrm{nm} \mathrm{Au}$ capping layer, the spin valve signal improves by a factor three compared with the uncapped devices. This improvement is due to the oxidation prevention of the Py electrode by Au capping layer [140]. The magnetoresistance increases with decreasing temperature. The junction resistance however depends weakly on temperature as observed in many organic spin valve work (Fig 4.7b) [32], [33], [74], [142], [143]. The charge transport in organic semiconductor is via thermally activated hopping which means junction resistance should increase significantly with decreasing temperature. This implies charge transport in these spin valve devices is dominated by hot-spots or other defects. Nevertheless, the measured magnetoresistance is caused by the presence of the organic semiconductor although meaningful spin dependent parameters cannot be extracted by this approach. The weak out of plane angular and bias dependence of spin valve signal suggests the measured signal is not a manifesto of TAMR (Fig $4.7 \mathrm{c}, \mathrm{d})$.

Patterning on top of organic semiconductors by optical lithography is very challenging as organic materials are prone to damage by patterning and lift-off processes. Micro-fabricated vertical organic spin valves were fabricated using the procedure discussed in Chapter 4.2.3. The junction resistance increases drastically with temperature as expected with hopping conduction (Fig 4.7e). The magnetoresistance measurement has suffered from significant noise fluctuation on the order of $2 \%$ (Fig $4.7 \mathrm{f}$ ). The presence of this noise is due to the nature of the hopping transport [54]. With the present level noise, it is impossible to resolve the signal observed in large area vertical spin valves. This measurement hints at there being pin holes in large area organic spin valves that are absent in small area junctions. 
a
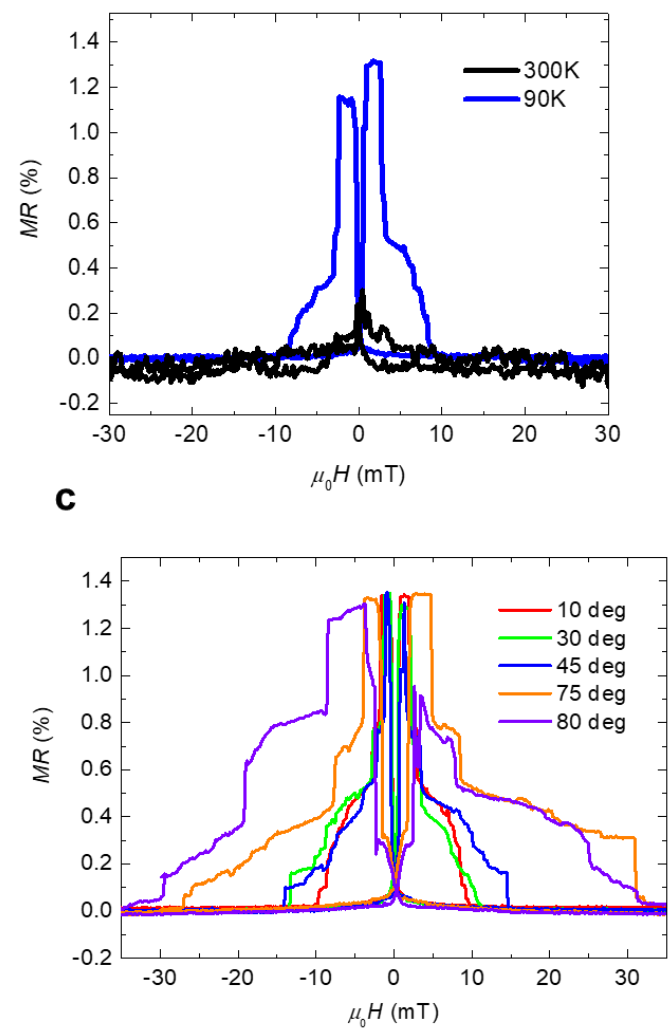

e

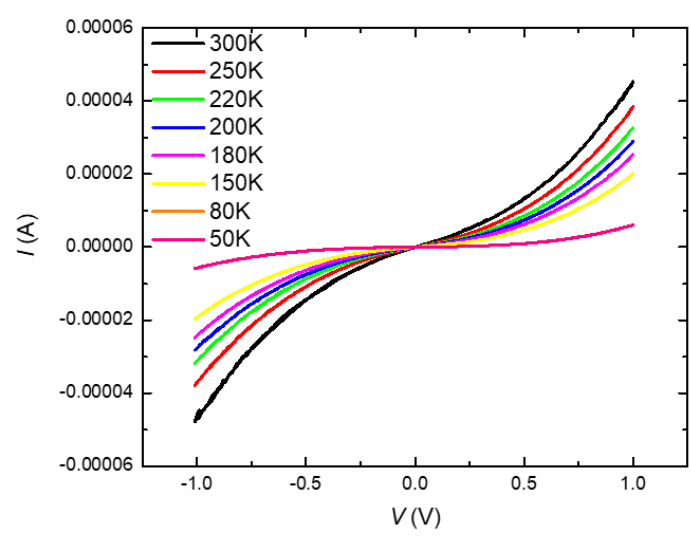

b

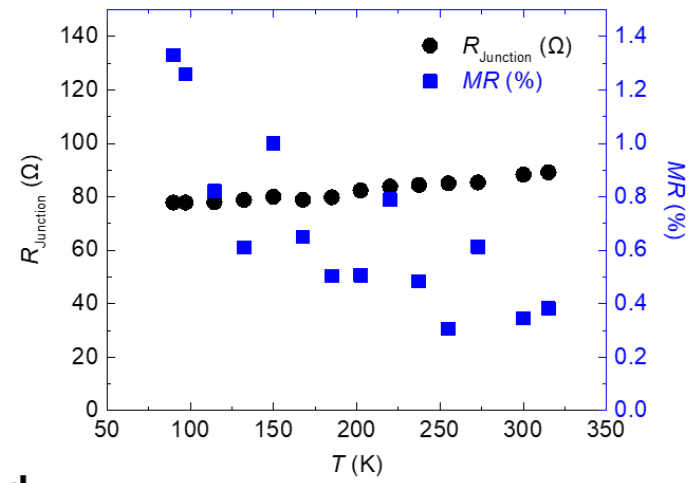

d

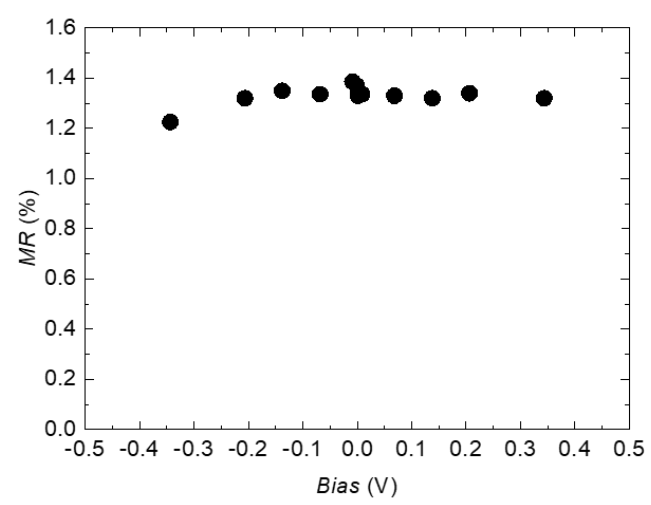

$\mathbf{f}$

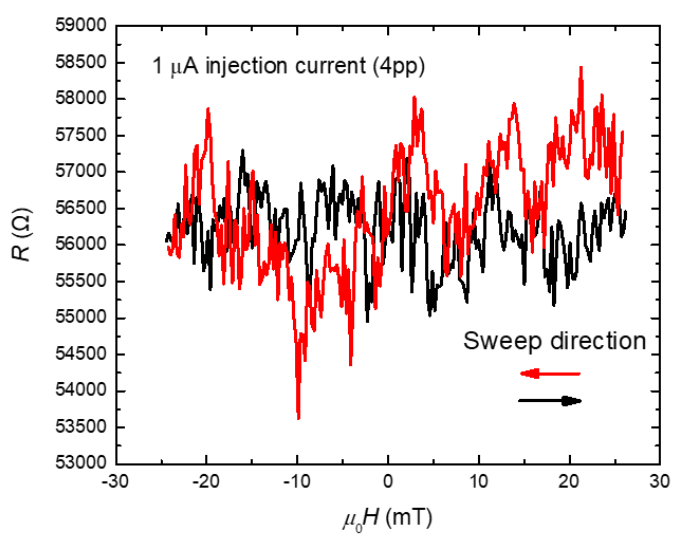

Figure 4.7 Magnetoresistance of vertical organic spin valve. a-d, Large area (200 x $200 \mu \mathrm{m})$ vertical organic spin valves. a, Spin valve effect observed for a non-shorted device at $300 \mathrm{~K}$ and $90 \mathrm{~K}$. b, Temperature dependence of spin valve signal and four-point junction resistance. c, Out of plane angular dependence of the measured spin valve signal at $90 \mathrm{~K}$. d, Bias dependence of the measured spin valve signal at $90 \mathrm{~K}$. The thickness of F4TCNQ-PBTTT is $75 \mathrm{~nm}$ and the applied current for the spin valve measurement is $100 \mu \mathrm{A}$. e-f, Microfabricated $(2 \times 5 \mu \mathrm{m})$ vertical organic spin valve measurement. e, Temperature dependence of the charge transport for microfabricated vertical spin valve device. $\mathbf{f}$, Spin valve measurement at $300 \mathrm{~K}$ with $1 \mu \mathrm{A}$ applied current. The thickness of F4TCNQ-PBTTT is $75 \mathrm{~nm}$. 


\subsubsection{Electrical spin injection and ISHE detection devices}

Fig 4.8a and $\mathrm{b}$ show the schematic and an optical micrograph of a nonlocal spintronic device architecture inspired by conventional dual-ferromagnet nonlocal spin valves, but with a difference in the detector scheme. In this device, an electrical current is injected at a ferromagnetic electrode (Py) and drifts towards the right gold electrode (Ohmic contact) under an applied bias. The detector constitutes a stripe of platinum nanofabricated a few hundred nanometres away from the ferromagnetic injector, with a Keithley $2182 \mathrm{~A}$ nanovoltmeter connected across it. Current injected through the ferromagnet is expected to generate a nonequilibrium spin polarisation in the organic semiconductor that diffuses isotropically, part of which is absorbed in the platinum detector.

The organic semiconductor used in this device is PBTTT doped with F4TCNQ to a conductivity of approximately $100 \mathrm{Scm}^{-1}$. Patterning of the organic semiconductor by optical lithography (shown as a dog bone pattern in Fig 4.8b) tends to reduce the conductivity value by a factor of two from the maximum doping concentration owing to the harsh processing that the patterning steps entail. Within the platinum, the absorbed spin current is converted into a transverse voltage measured across it due to a strong spin-orbit coupling induced inverse spin Hall effect within the platinum stripe. Such a non-local device architecture in which a spin polarised current is electrically injected into the active layer and detected using the inverse spin hall effect (ISHE) in a heavy metal was used previously to unambiguously probe spin diffusion in metal based nonlocal spin valves.

Fig 4.8c shows the expected measurement outcome for this measurement assuming successful spin current transmission in the spin transport material and the spin current absorbed by heavy metal strip. The ISHE signal changes sign with the magnetisation of the Py electrode due to ISHE symmetry which results in a $2 \Delta R_{\text {ISHE }}$ difference between positive and negative magnetisation direction. The idea of using this architecture for probing spin transport in organic semiconductors originates from the possible advantage of spin current detection over spin accumulation detection in conventional non-local spin valves. PBTTT has a highest occupied molecular orbital (HOMO) of $5.1 \mathrm{eV}$ whereas Au has a work function of $5.1 \mathrm{eV}$. This band alignment makes $\mathrm{Au}$ an ideal charge injection material for PBTTT. Fig 4.8d-f compare the charge injection into doped PBTTT using various contact electrodes (Pt, $3 \mathrm{~nm} \mathrm{Au}$ capped Py and $\mathrm{Au}$ ). It is important to note that $3 \mathrm{~nm}$ Au capped Py electrode injects carriers into PBTTT 
as effectively as the Au electrode. This means the Au capping layer is conformal on top of Py and no native Py oxide is formed between Au capped Py electrode and doped PBTTT.

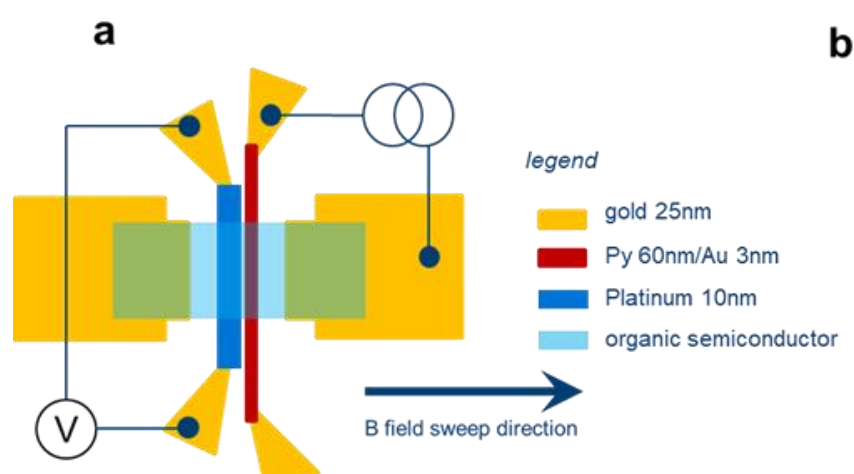

C

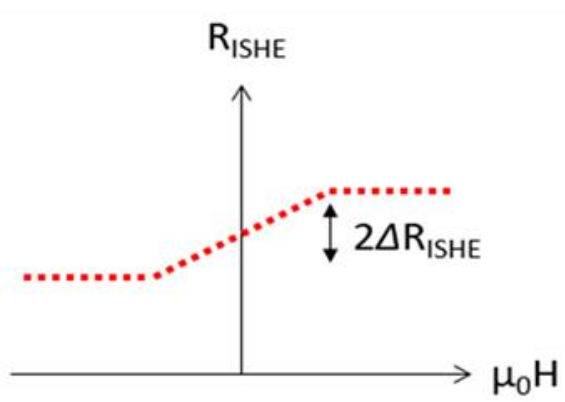

b
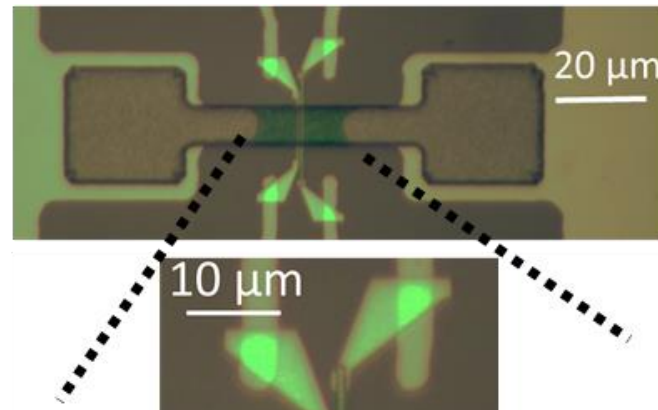

d

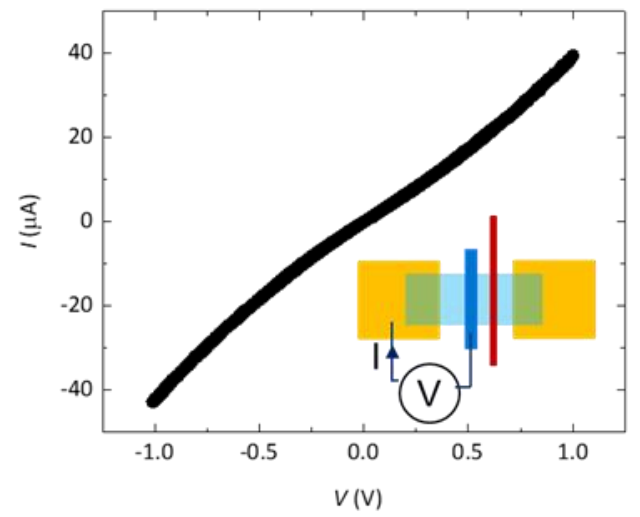

f

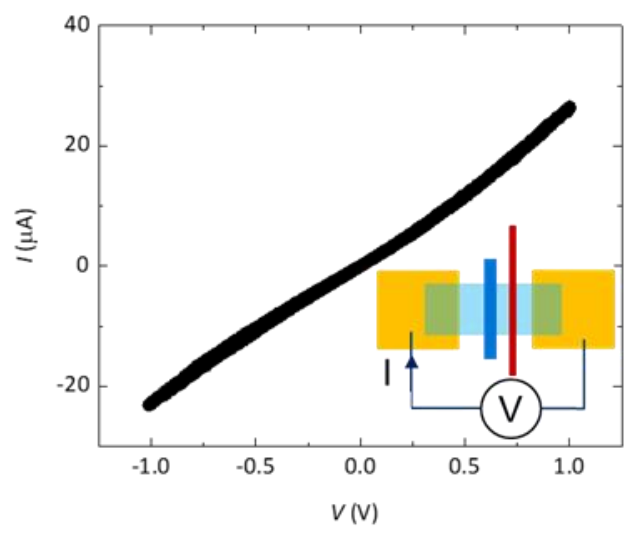

Figure 4.8 Electrical spin injection into organic semiconductor and ISHE detection. a, Schematic electrical spin injection and ISHE detection measurement configuration. Diffusive spin current is generated by electrical spin injection from ferromagnetic electrode. The transmitted pure spin current 
in organic semiconductor is detected by ISHE of Pt in close proximity with the ferromagnetic electrode.

b, Optical image of the electrical spin injection and ISHE detection device. The bottom panel shows an enlarged image of the active device region. $\mathbf{c}$, Expected measurement outcome for the voltage across the Pt wire as a function of applied magnetic field. d-f, Current-voltage (I-V) characteristics of the PtF4TCNQ doped PBTTT-Au (d), $3 \mathrm{~nm}$ Au capped Py-F4TCNQ doped PBTTT-Au (e), Au-F4TCNQ doped PBTTT-Au (f). The insets show the measurement configurations for the $I-V$ measurements.

Fig 4.9a shows a typical measurement where the voltage is probed across the Pt detector while sweeping the magnetic field on three different devices with an injector (Py) to detector $(\mathrm{Pt})$ spacing of $100 \mathrm{~nm}, 200 \mathrm{~nm}$ and $400 \mathrm{~nm}$. The gap spacing was chosen to be on the order of magnitude of the reported spin diffusion length from tri-layer organic spin valves. No signature of a generated inverse spin hall effect signal is observed in any of these devices. In addition, the offset voltage across the detector electrode depends on how close it is to the injector. As shown in Fig $4.9 \mathrm{~b}$ for a fixed injected current of $1 \mu \mathrm{A}$, both the offset and measured noise fluctuations reduce when the Pt detector is moved further away from the Py injector. This indicates there being an electrical cross-talk between the Py injector and Pt detector. In other words, the injected current from the ferromagnetic injector spreads into the platinum detector via the patterned conductive organic semiconductor.

The voltage noise fluctuations on the offset (shown as error bars) also increase for smaller distances between the Pt detector and the Py injector, and is attributed to the charge transport in the organic semiconductor. The very nature of hopping conduction in the organic semiconductor generates flicker noise as the current spreads into it before being picked up by the detector causing the noise to be superimposed on the detected voltage across platinum. Furthermore, when the measurement is performed in $\mathrm{AC}$, the noise of the measurement is very sensitive to the measurement frequency (noise decrease with increasing frequency) which is a clear indication of $1 / f$ noise that originates from stochastic hopping transport. To further validate the effect of electrical cross-talk, we send a current through the ferromagnetic electrode and probe the voltage across the Pt detector (thermal spin injection and inverse spin hall effect detection geometry). The results of this are shown in Fig 4.9c and $\mathrm{d}$ for a device with a channel length of $100 \mathrm{~nm}$. Here, as the electrical current is increased through the ferromagnet, both the offset voltage and the superimposed voltage noise fluctuations increase.

In both a non-local spin valve geometry as well as in our electrical spin injection with ISHE 
detection geometry, a larger spin accumulation at the injection electrode is achieved by increasing the current through it. If, however, this causes noise fluctuations at the detector that is on the order of tens to a hundred microvolts as is often the case, a chance of measuring a spin accumulation at the detector electrode on the order of a ten to a hundred $\mu \mathrm{eV}$ is completely ruled out. This clearly reinforces our claim that as the current travels through the ferromagnet, part of it spreads into the nonlocal platinum detector mediated by a conductive pathway in the organic semiconductor where it picks up noise fluctuations due to the nature of hopping conduction. Such a current spreading phenomena in nanofabricated nonlocal spin injection architectures using hopping conductors is an intrinsic roadblock towards the successful implementation of electrical injection with nonlocal detection in organic semiconductors. 
a

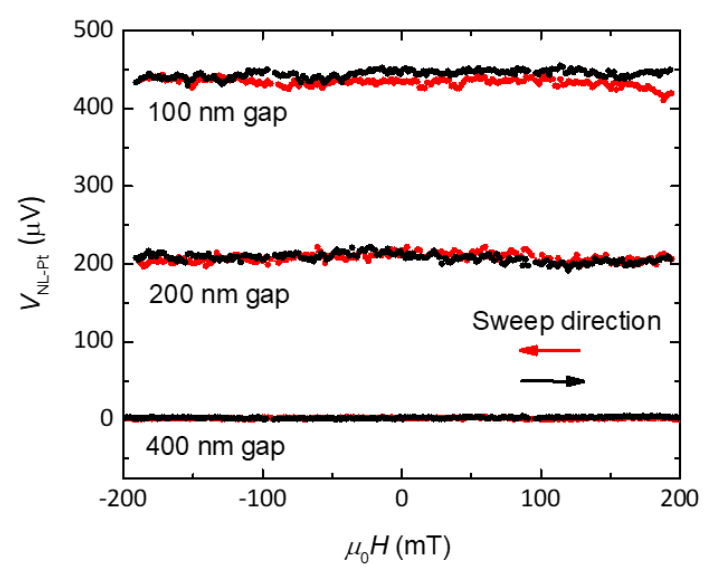

C

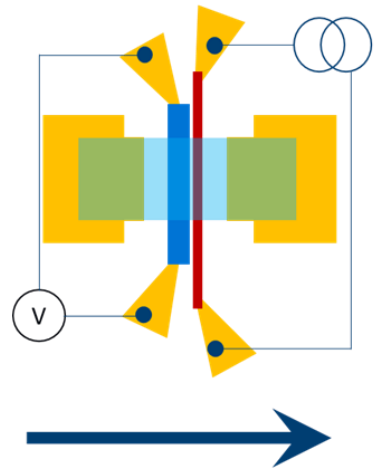

B field sweep direction b

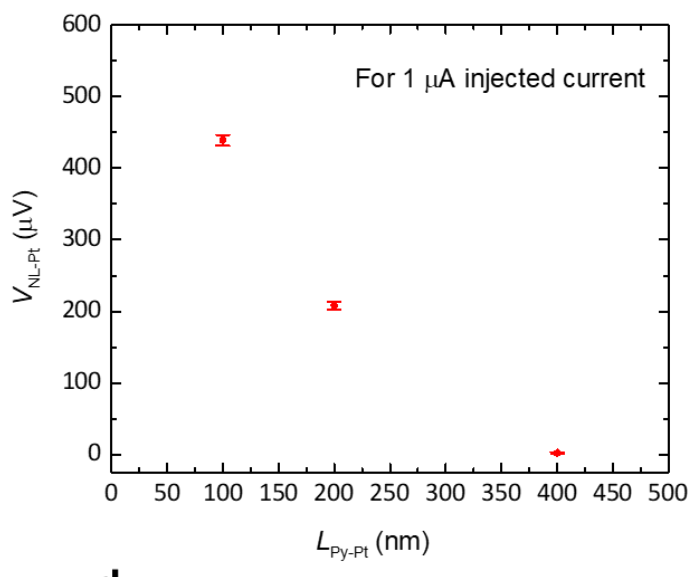

d

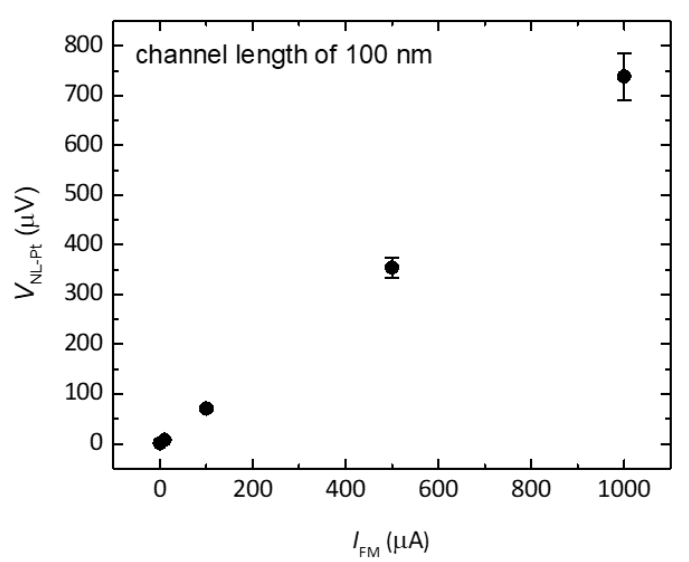

Figure 4.9 Electrical spin injection and ISHE detection measurement-current spreading effect. a, Electrical spin injection and ISHE detection measurements with different gap spacing between Py and Pt. The injected current is $1 \mu \mathrm{A}$. b, Gap spacing between Py and Pt dependence of the non-local voltage across Pt. The measurement noise is superimposed on the voltage magnitude as error bar. c, Thermal spin injection and ISHE detection. Thermal spin injection into the organic semiconductor is achieved via applying a DC current through Py electrode. This current generates a temperature difference at the interface between Py and OSC which leads to thermal spin injection. d, Current through Py dependence of non-local voltage across Pt. The gap spacing between Py and Pt is $100 \mathrm{~nm}$. 


\subsection{Conclusions}

Non-local spin valves based on high mobility organic semiconductors have been studied systematically through tuning the interface charge injection properties and moving to novel device architectures specific to organic conjugated polymers. Both the enhancement in spin accumulation in out of plane spin injection non-local spin valves and the pure spin current detection method in electrical spin injection and ISHE detection devices did not lead to successful measurement of lateral spin transport in organic semiconductors. However, the current spreading effect and the hopping conduction in organic semiconductors were experimentally verified as the intrinsic roadblocks in electrical spin injection and detection measurement for organic semiconductors. Even though a magnetoresistance signal has been detected in large area $(200 \times 200 \mu \mathrm{m})$ doped PBTTT/F4TCNQ vertical spin valve with $3 \mathrm{~d}$ ferromagnetic electrodes in the absence of tunnel barrier, the magnetoresistance signal vanished with reduced junction area $(2 \times 5 \mu \mathrm{m})$ which indicates the signal does not originates from coherent spin transport [131].

Despite the intrinsic roadblocks for electrical spin injection and detection in organic semiconductors, it may not be possible to detect spin transport in organic semiconductors using electrical spin injection and detection. There are some possible ways to achieve this for example, use high mobility rubrene single crystal/PFPE with "band-like" charge transport that shows ultra-low noise in Hall effect measurement [144] (Fig 4.10a and b) and/or design novel non-local spin transport architecture that the current spreading effect is minimised through device design. 
a

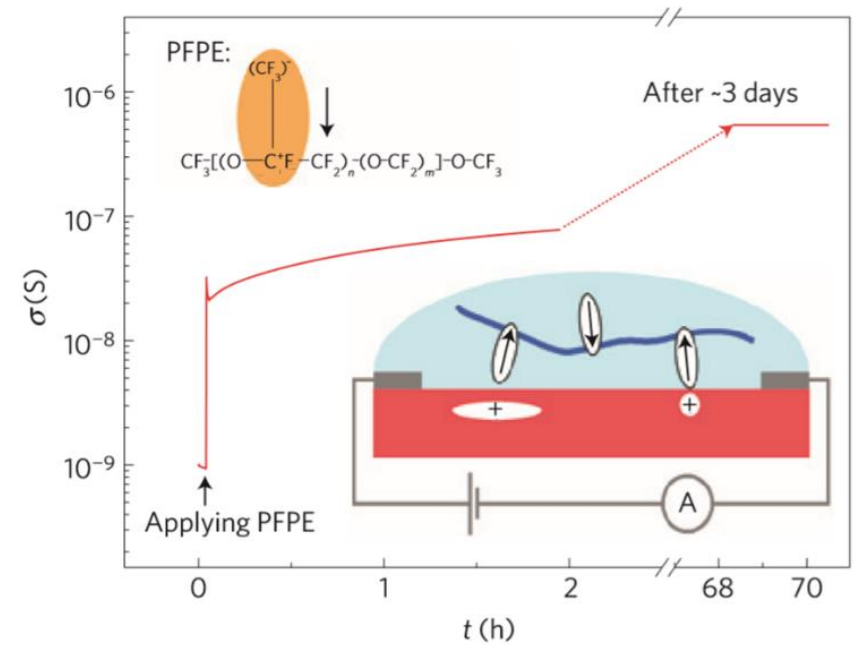

b

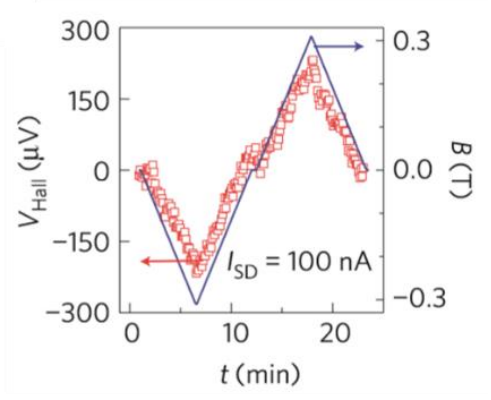

Figure 4.10 Ultralow noise Hall effect in rubrene signal crystal/PFPE. a, Surface conductivity, $\sigma$ of rubrene single crystal as a function of the time after PFPE is dropped on top of rubrene single crystal as shown in the inset. $\mathbf{b}$, Hall effect measurement of the rubrene single crystal functionalised with PFPE. The excitation current is $100 \mathrm{nA}$. This figure is modified from [144]. 


\section{Chapter 5}

\section{Vertical spin pumping into organic semiconductors}

\subsection{Motivation}

The combination of FMR induced spin pumping and ISHE is becoming a popular experimental technique to study spin dynamics in a wide variety of materials from metals to inorganic semiconductors due to the ease of fabrication and measurement as well as the ability to circumvent conductivity mismatch problem by spin pumping. The field of organic spintronics has been primarily limited to vertical spin valves for understanding spin transport in organic semiconductors. The on-going debate about the potential artefacts in conventional vertical organic spin valves such as TAMR can be avoided in spin pumping experiment. The observation of spin to charge conversion in PEDOT: PSS by FMR spin pumping and the more recent observation of spin transport in PBTTT by spin pumping and ISHE of a heavy metal detector have opened up a new avenue to probe spin dynamics in organic materials by using spin pumping technique [61], [62], [145], [146], [147], [148], [149]. This chapter explores the spin transport in a variety of organic semiconductors using tri-layer spin pumping architecture where the organic semiconductor is sandwiched between a ferromagnetic layer under ferromagnetic resonance and a heavy metal detector layer. The pure spin current injected into the organic semiconductor by spin pumping is converted into a charge current via ISHE of the heavy metal layer. In particular, the spin transport properties of organic semiconductors are correlated with the molecular structure and charge transport properties of the organic semiconductors. 


\subsection{Pure spin current transport in organic semiconductors using tri-layer spin pumping architecture at room temperature}

Fig 5.1a shows a schematic illustration of the tri-layer spin pumping structure and the measurement geometry. The Py layer is driven into FMR by the applied microwave which injects a flow of pure spin current into the organic semiconductor in contact with Py by spin pumping. The spin current diffuses through the organic semiconductor in the vertical direction and the spin current that reaches the Pt detector is converted into charge current via ISHE of Pt. This ISHE induced current is probed as electrical voltage. FMR linewidth broadening is often used to quantify the amount of spin current injection from the ferromagnet into the nonmagnet in contact where FMR spectra of the ferromagnet in proximity with the non-magnet is compared with the FMR spectra of the ferromagnet. However, much care is required for such analysis since there could be other origins that causes FMR linewidth broadening other than spin current injection. Py/Pt device is often taken as the reference for FMR linewidth broadening measurement due to Pt being a strong spin sink that allows efficient spin current injection into Pt and hence significant FMR linewidth broadening. The FMR linewidth for the $\mathrm{Py} / \mathrm{PBTTT} / \mathrm{Pt}$ device is even broader than the Py/Pt device (Fig 5.1b). However, this may not necessarily mean the spin current injection into organic semiconductor is more efficient than the case of $\mathrm{Py} / \mathrm{Pt}$ since the surface roughness of the polymer leads to significant inhomogeneous broadening of linewidth for Py layer deposited on top of the polymer. As a consequence, an accurate estimation of the spin current injection cannot be obtained by using the FMR linewidth broadening when the surface morphologies of the non-magnetic materials beneath the ferromagnetic layer are different. 
Charge transport in the vertical direction of the organic semiconductor can be examined by a vertical diode geometry where organic semiconductor is sandwiched between top and bottom electrodes in a cross-bar geometry. Under low bias, the conduction is mediated by the residual carriers in the polymer film which shows a linear current-voltage characteristics (Ohmic). As the bias increases beyond a threshold voltage, the current-voltage characteristics follows the Mott-Gurney relationship [Eq. (5.1)] due to the formation of space charge limited current in organic polymer [150], [151].

$$
J_{\mathrm{SCLC}}=\frac{9}{8} \varepsilon_{0} \varepsilon_{\mathrm{r}} \mu \frac{V^{2}}{d^{3}}
$$

where $\varepsilon_{0}, \varepsilon_{r}, \mu$ and $d$ denote the free space permittivity, relative permittivity, organic semiconductor out of plane mobility and the thickness of the polymer film, respectively. Fig 5.1c shows the current density dependence on the electric field for PBTTT with different thickness using Py and Pt as contact electrodes. Due to the well alignment between the HOMO level of PBTTT and metal electrodes work functions, this diode transports holes only. Following the SCLC analysis with holes injection from Py electrode, out of plane mobilities of PBTTT and P3HT are estimated to be $1 \times 10^{-5} \mathrm{~cm}^{2} \mathrm{~V}^{-1} \mathrm{~s}^{-1}$ and $2 \times 10^{-4} \mathrm{~cm}^{2} \mathrm{~V}^{-1} \mathrm{~s}^{-1}$, respectively. In particular, the current density scales accordingly with the thickness of the PBTTT (Fig 5.1c) for ideal SCLC charge transport confirming the formation of space charge in PBTTT. In contrast, despite the HOMO level of DPP-BTZ aligning well with the work functions of the contact electrodes [152], the $J-V$ characteristics for hole-only DPP-BTZ diodes with different thicknesses essentially follow the same curve when normalising the $V$ with the thickness of DPP-BTZ. This provides clear evidence that the charge transport in DPP-BTZ is injection (contact) limited [153]. Considering the well alignment of the HOMO level of DPP-BTZ with the charge injection Py electrode, the presence of contact limited charge transport is most likely caused by high trap density present in the DPP-BTZ film which results in high contact resistance [154]. 


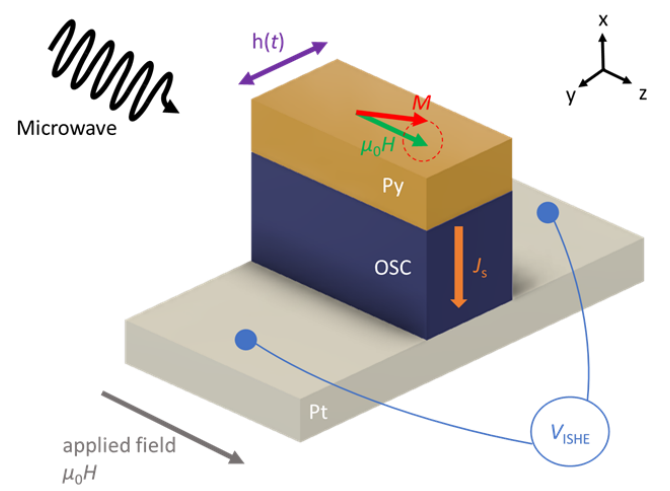

C

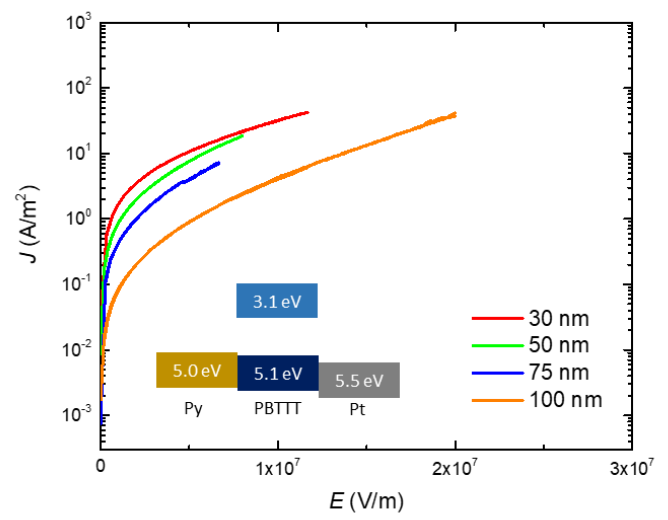

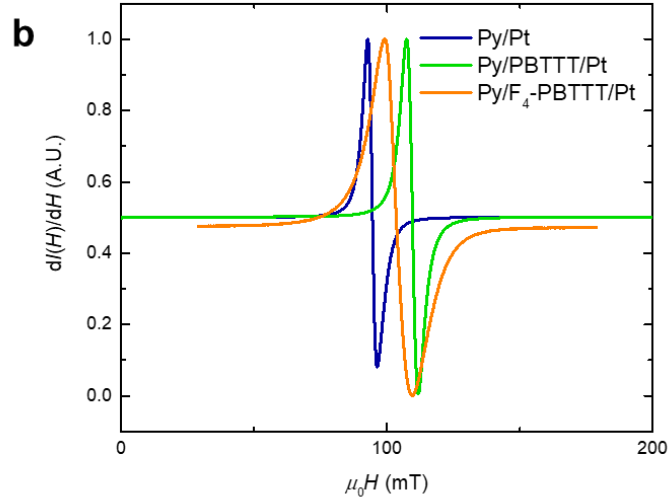

d

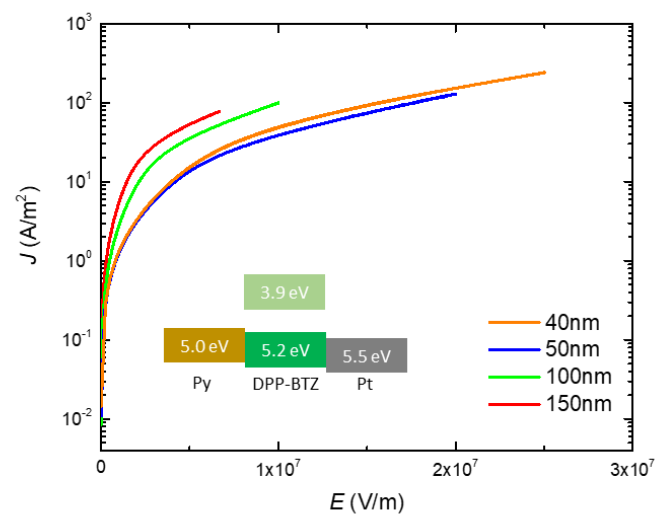

Figure 5.1 Tri-layer spin pumping measurements. a, Schematic illustration of the tri-layer spin pumping architecture and measurement configuration. Py stripe is excited into FMR by the microwave which injects pure spin current into the adjacent organic semiconductor film. The spin current that arrives at the Pt layer is converted into a charge current via ISHE. b, Field $\left(\mu_{0} H\right)$ dependence of the FMR signals $d I(H) / d H$ measured for Py/Pt (blue line), Py/PBTTT/Pt (green line) and Py/doped PBTTT/Pt (orange line) at $100 \mathrm{~mW}$ microwave excitation and $9.38 \mathrm{GHz}$ microwave frequency inside an ESR cavity where $I$ is the microwave absorption intensity. The external magnetic field is applied parallel to the Py film, but perpendicular to the long axis of the platinum detector. c, The current density, $J$ dependence of the electric field, $E$ for Py/PBTTT/Pt diodes with different PBTTT thicknesses. d, The current density, $J$ dependence of the electric field, $E$ for Py/DPP-BTZ/Pt diodes with different DPPBTZ thicknesses. The active junction area is $200 \times 200 \mu \mathrm{m}$ and holes are injected into the organic semiconductor from Py contact. The inset shows the band alignment between the HOMO level of the organic semiconductor and work functions of the injection metals. 
Fig 5.2a shows the field dependence of the ISHE induced current for reference Py/Pt bilayer and tri-layer Py/PBTTT/Pt and Py/doped PBTTT/Pt devices. Clearly, the ISHE signal is the highest for the Py/Pt device (around $260 \mathrm{nA}$ ) while the signal reduces by a factor of ten by insertion of a $50 \mathrm{~nm}$ PBTTT layer between Py and Pt electrodes. The ISHE signal increases by a factor of two upon doping PBTTT fully with F4TCNQ (Fig 5.2a). Fig 5.2b shows the vertical conductivity of PBTTT as a function of doping level using a cross-bar geometry with Au injector and detector electrodes (the doping level increases from 1 to 3). Py/P3HT/Pt device shows a similar ISHE current signal compared to the case of Py/PBTTT/Pt device (Fig 5.2c). In contrast, no such signal can be measured for Py/DPP-BTZ/Pt device (Fig 5.2d). From a charge transport point of view, DPP-BTZ possesses higher charge carrier mobility than both PBTTT and P3HT in the in-plane direction. In addition, DPP-BTZ orientates face-on with respect to the substrate plane which provides high mobility in the out of plane direction. The reason for DPP-BTZ device not showing a ISHE signal is hence most likely to be caused by the high trap density present in the film that originates from film processing conditions, top electrode deposition (formation of thermally induced traps) and the intrinsic trap density in DPP-BTZ. In comparison, it is known that both PBTTT and P3HT have low trap density on the order of $10^{13} \mathrm{~cm}^{-2}$ [155], [156].

In addition, the ability to deposit heavy metal such as Pt on top of organic semiconductor without damaging the organic semiconductor is essential for a number of spintronics measurements such as optical spin injection and ISHE detection where high quality optical spin source needs to be prepared before deposition of organic semiconductor. Due to the extremely high boiling points of heavy metal elements, it is very challenging to thermally evaporate these materials without burning the organic semiconductors. Moreover, e-beam evaporation of heavy metals on top of organic semiconductors tends to thermally damage organic semiconductors due to high energy electron beam. To mitigate the thermal damage imposed by evaporation methods, magnetron sputtering based on momentum transfer to "sputter" the target material potentially offers a solution to this obstacle. However, the energy of sputtered atoms depends strongly on sputtering pressure. The lower the sputtering pressure, the higher the energy of the sputtered atoms which tends to penetrate the soft organic semiconductors. As such, the sputtering parameters have been optimised (sputtering pressure $=1 \times 10^{-1} \mathrm{mbar}$ with pure argon flow of $3.6 \mathrm{sccm}$, sputtering power $=100 \mathrm{~W}$ with $\mathrm{rf}$ sputtering) to successfully deposit $\mathrm{Pt}$ on PBTTT without damage PBTTT as confirmed by efficient charge injection from Pt electrode in Pt/PBTTT/Au diode measurements. However, due to the morphology of Pt prepared under 
these conditions (less dense than normal sputtering conditions), the ISHE signal is reduced by a factor of five compared to Pt prepared under normal sputtering conditions as confirmed by $\mathrm{Py} / \mathrm{Pt}$ bilayer spin pumping measurements.
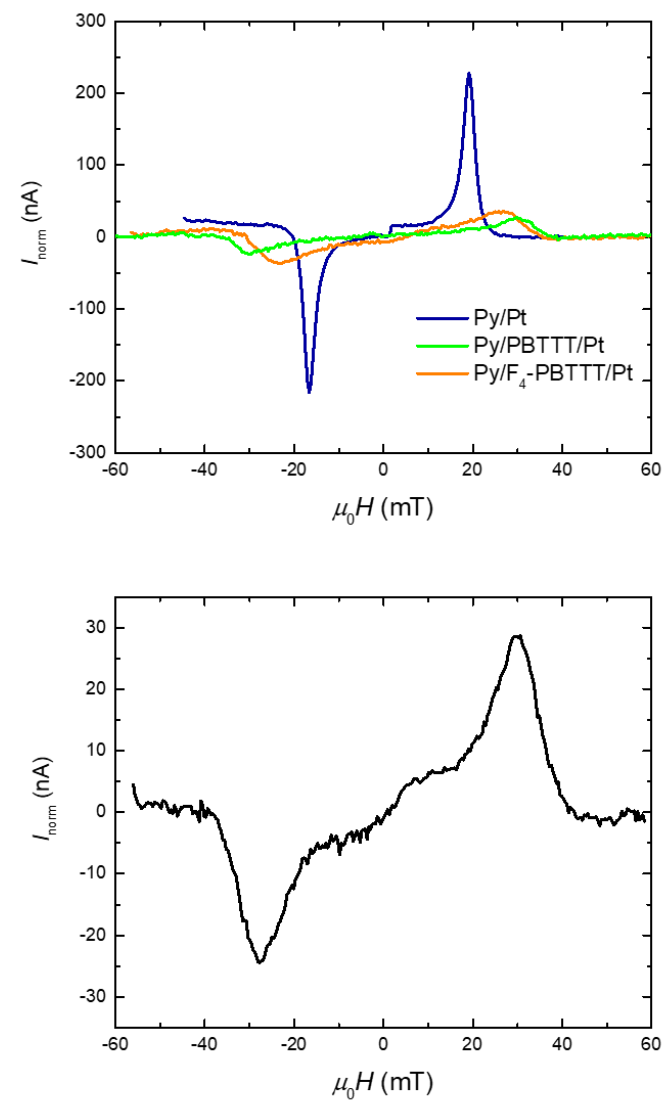

b
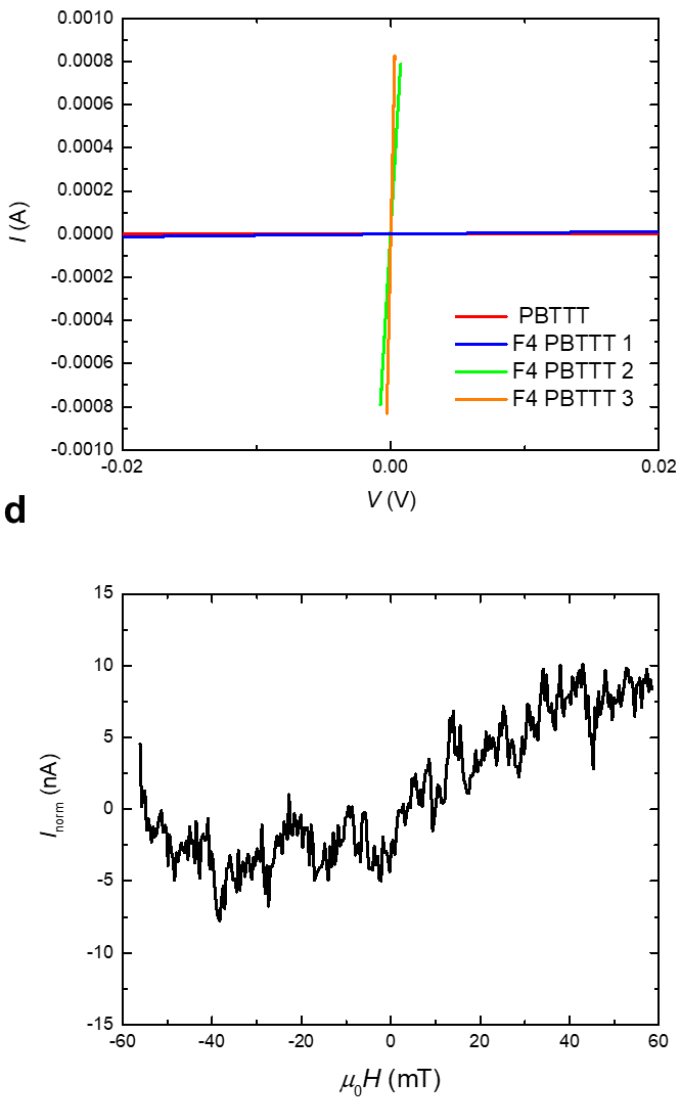

Figure 5.2 Pure spin current transport in organic conjugated polymers at room temperature. a,

Field $\left(\mu_{0} H\right)$ dependence of the ISHE induced current, $I_{\text {norm }}$ measured for Py/Pt (blue line), Py/PBTTT/Pt (green line) and Py/doped PBTTT/Pt (orange line). b, $I-V$ characteristics of Au/PBTTT/Au diodes with different levels of doping. The level of doping increases from 1 to 3 and the active junction area is 200 x $200 \mu \mathrm{m}$. c-d, Field $\left(\mu_{0} H\right)$ dependence of the $I_{\text {norm }}$ measured for Py/P3HT/Pt (c) and Py/DPP-BTZ/Pt (d). For spin pumping measurement, the microwave power applied is $1 \mathrm{~W}$ and the microwave frequency is $4 \mathrm{GHz}$. The thickness of the organic conjugated polymers is around $50 \mathrm{~nm}$. 
The ISHE induced current increases weakly with the out of plane conductivity of PBTTT where the ISHE signal increases by a factor of two when the conductivity increases by orders of magnitude (Fig 5.3a). This is a surprising result since high conductivity corresponds to high carrier density at the interface between the ferromagnet and organic semiconductor that mediates the spin current transmission across the organic semiconductor. This result implies the possibility of efficient exchange interaction between the polarons in the out of plane direction where polymer chains are stacked edge-on to the substrate plane. In the edge-on molecular stacking, the polymer chains are isolated from each other by the insulating alkyl side chains in the out of plane direction. This is the reason why P3HT have a higher carrier mobility than PBTTT in the out of plane direction due to the shorter side chain $\left(\mathrm{C}_{6}\right.$ for P3HT and $\mathrm{C}_{14}$ for PBTTT). Fig 5.3b shows a thickness dependence of the ISHE current for P3HT devices. The spin diffusion length of P3HT in the out of plane direction is estimated to be $596 \pm 61 \mathrm{~nm}$ by fitting an exponential function is fitted to the data. Spin diffusion lengths for PBTTT with different levels of doping can be extracted using the same method (Fig 5.3c). The spin diffusion length for PBTTT clearly increases with the doping level (Fig 5.3d) from around $130 \mathrm{~nm}$ to $600 \mathrm{~nm}$. This four-fold increase in spin diffusion length implies an exchange-based spin transport in doped PBTTT where the high carrier concentration allows greater overlap between the delocalised polarons leading to enhancement in spin diffusion length. The longer spin diffusion length of P3HT compared to PBTTT could be due to either the higher out of plane mobility for P3HT than PBTTT and/or enhanced exchange interaction between the polarons as a result of side chain length reduction (polymer cores closer to each other).

Two control experiments were performed to verify tri-layer spin pumping experiments. First, an organic insulator, Poly(methyl methacrylate) (PMMA) was inserted between Py and Pt instead of organic semiconductor to block spin current transmission between Py and Pt. No ISHE signal can be observed for Py/PMMA $(50 \mathrm{~nm}) / \mathrm{Pt}(10 \mathrm{~nm})$ device (Fig 5.4a) which confirms the ISHE signal is due to spin current transport across Py and Pt mediated by charge transport. Second, the Pt detector was replaced by Al layer with negligible spin-orbit coupling to confirm the ISHE signal is generated by the Pt detector via its high spin-orbit interaction. Similarly, no ISHE signal can be observed for Py/P3HT (50 nm)/Al (10 nm) device (Fig 5.4b) which verified the ISHE signal is generated by the Pt detector. 
a

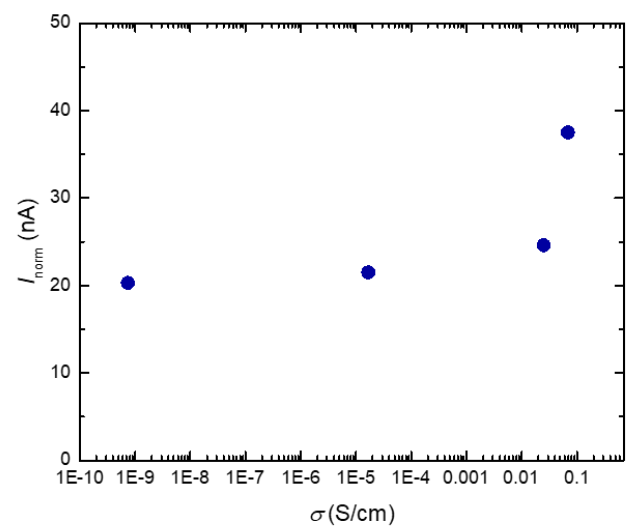

C

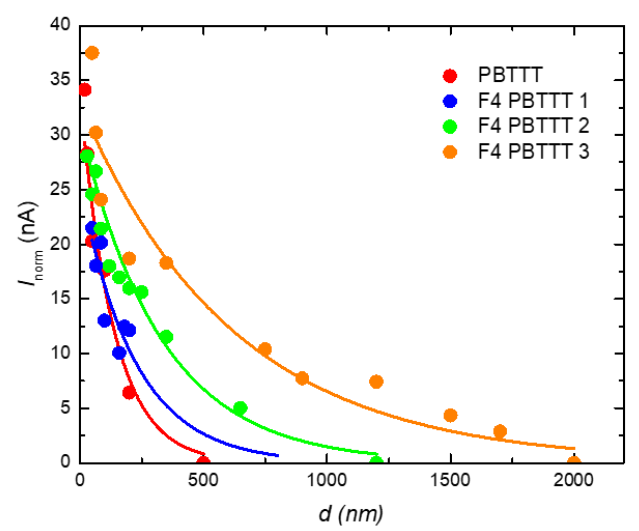

b

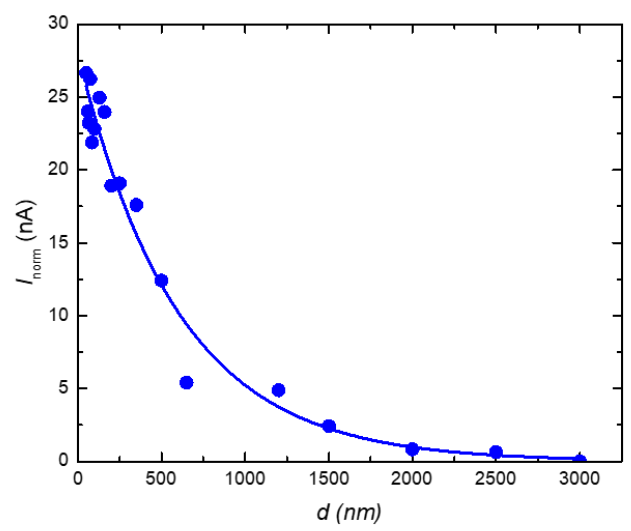

d

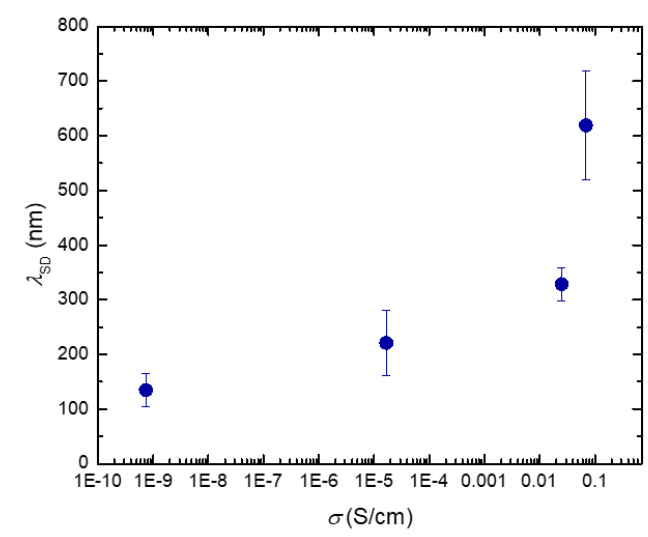

Figure 5.3 Spin current transmission in conjugated organic polymers at room temperature. a, Conductivity, $\sigma$ dependence of the ISHE induced current, $I_{\text {norm }}$ for doped PBTTT. b-c, Thickness, $d$ dependence of $I_{\text {norm }}$ for P3HT (b) and PBTTT with different levels of doping (c). The level of doping increases from 1 to 3 [the conductivity increases from $1.7 \times 10^{-5} \mathrm{~S} / \mathrm{cm}$ (doping level 1) to $0.07 \mathrm{~S} / \mathrm{cm}$ (doping level 3)] and the solid lines are exponential fits to experimental data. d, Conductivity dependence of spin diffusion length extracted from exponential fits for PBTTT. The error bars indicate the fit uncertainty. 


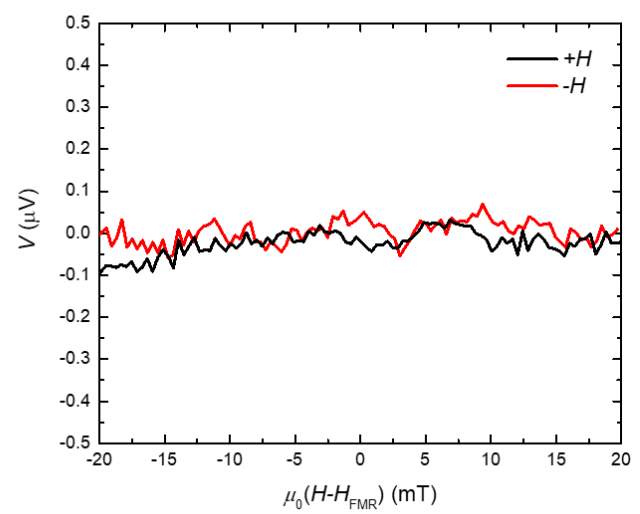

b

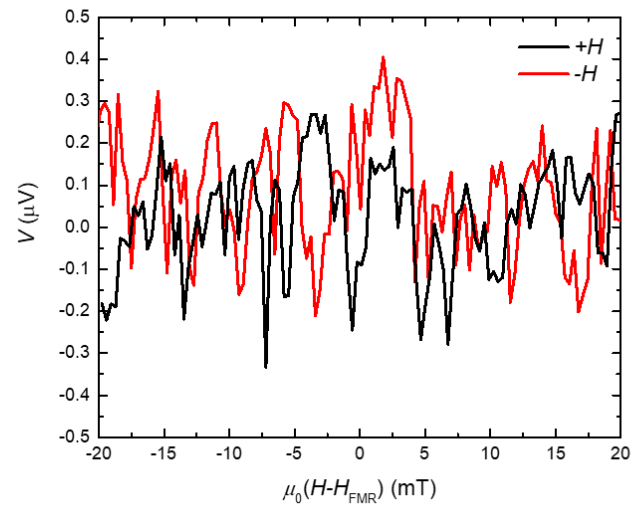

Figure 5.4 Tri-layer spin pumping control experiments. a-b, Field dependence $\left(\mu_{0} H\right)$ of the electromotive force, $V$ measured for Py/PMMA (50 nm)/Pt (10 nm) (a), Py/P3HT (50 nm)/Al (10 nm) (b). The microwave power applied is $1 \mathrm{~W}$ and the microwave frequency is $4 \mathrm{GHz}$. All measurements were performed at room temperature.

Out of plane angular dependence of the ISHE signal measurements were performed to understand the spin dynamics in these organic semiconductor systems. Fig 5.5a shows the measurement configuration and the relationship between the external field angle and the magnetisation angle where the magnetisation angle $\phi$ is calculated from the $\theta$ dependence of FMR resonance field, $H_{\text {FMR }}$ using Landau-Lifshitz-Gilbert (LLG) equation. Clearly, the ISHE voltage signal, $V_{\mathrm{ISHE}}$ vanishes at $\theta=90^{\circ}$ and inverts in sign at $\theta=180^{\circ}$, in good agreement with ISHE symmetry (Fig 5.5b). Following the theoretical work developed by Watanabe et al for Py/PBTTT/Pt system, the $V_{\text {ISHE }}$ as a function of $\theta$ for Py/PBTTT $(50 \mathrm{~nm}) / \mathrm{Pt}(10 \mathrm{~nm})$ was simulated theoretically for different spin lifetimes from ps to $\mu \mathrm{s}$. There are essentially two distinct regimes, for long spin lifetime greater than $1 \mu \mathrm{s}$, the angular dependence is more "gradual" while for short spin lifetime less than 1 ps, the angular dependence is more "steplike". The former is often regarded as the "Hanle" type angular dependence which resolves the spin precession in an out of plane magnetic field. The more "gradual" angular dependence for pristine PBTTT and pristine P3HT (Fig 5.5d) evolves to a more "step-like" angular dependence for doped PBTTT implies the suppression of Hanle effect due to enhanced exchange interaction between polarons consistent with recent proposed theory and experiment. It is important to note that the observed gradual angular dependence may be caused by factor other than Hanle effect such as non-uniform microwave magnetic field [145]. Therefore, one needs to be careful about using the out of plane angular dependence as the proof of Hanle effect in organic semiconductors (discussed in more details in Chapter 6). 
a

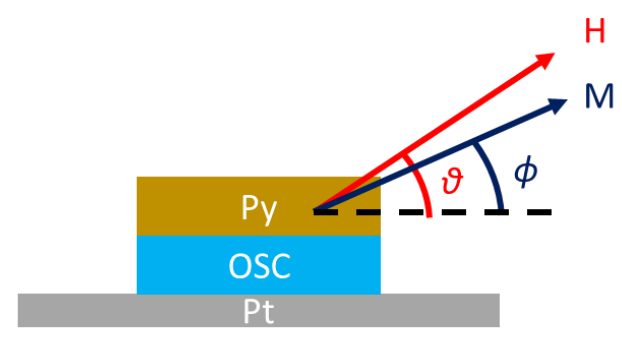

C

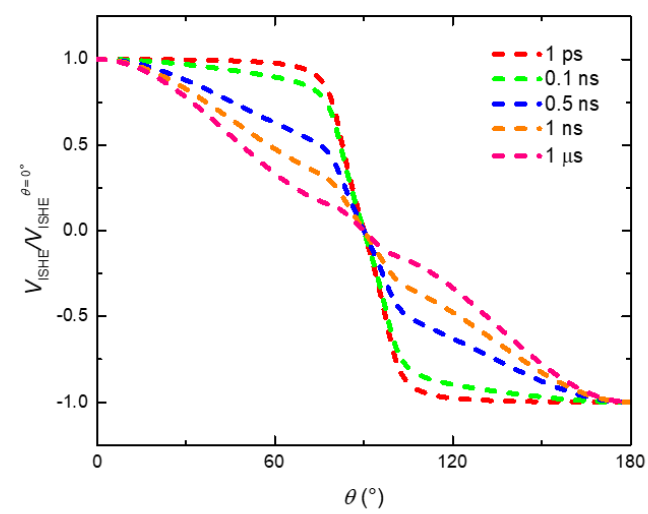

b
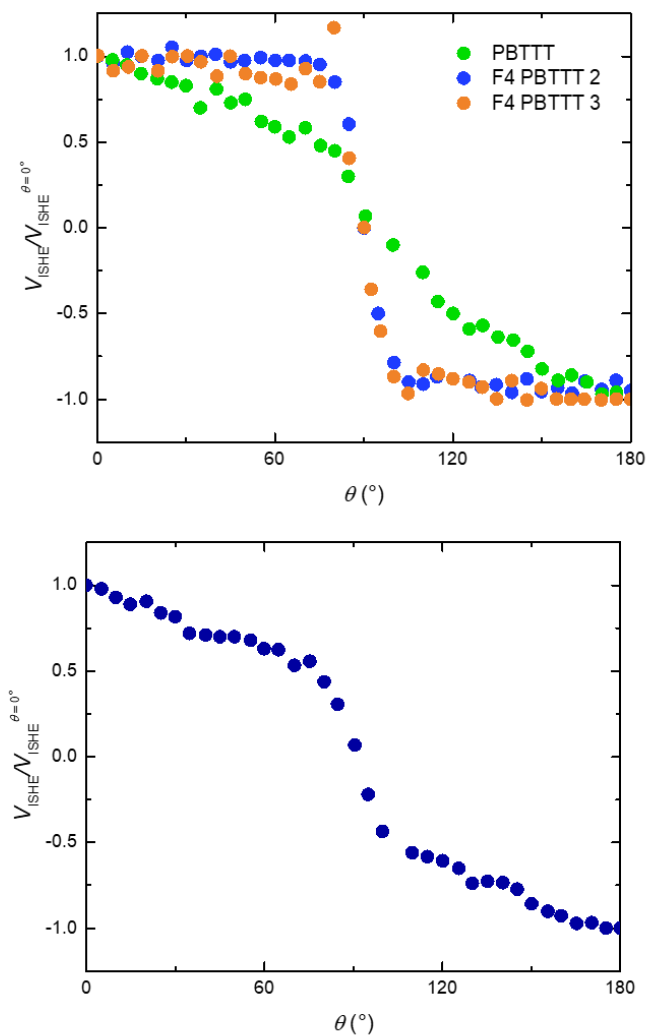

Figure 5.5 Out of plane angular dependence of the ISHE signal in the tri-layer spin pumping devices at room temperature. a, Schematic illustration of the angular dependence measurement configuration and the relationship between the external field angle and the magnetisation angle. $\mathbf{b}$, The ISHE voltage, $V_{\text {ISHE }}$ measured as a function of out of plane magnetic field angle, $\theta$. The green, blue and orange solid circles are the experimental data for Py/PBTTT $(50 \mathrm{~nm}) / \mathrm{Pt}(10 \mathrm{~nm})$, Py/intermediate doped PBTTT (50 nm)/Pt (10 nm) and Py/full doped PBTTT (50 nm)/Pt (10 nm), respectively. c, Simulated $V_{\text {ISHE }}$ as a function of $\theta$ for Py/PBTTT $(50 \mathrm{~nm}) / \mathrm{Pt}(10 \mathrm{~nm})$ for different spin lifetimes. d, The $V_{\text {ISHE }}$ measured as a function $\theta$ for $\mathrm{Py} / \mathrm{P} 3 \mathrm{HT}(50 \mathrm{~nm}) / \mathrm{Pt}(10 \mathrm{~nm})$. 


\subsection{Conclusions}

Tri-layer spin pumping experiment has been used to study the spin transport in a range of organic semiconductors with different molecular structure and charge transport properties. In particular, the doping dependence of the spin pumping signal was investigated for F4TCNQ/PBTTT system. The spin diffusion length of PBTTT was found to increase with doping concentration. Furthermore, the out of plane angular dependence measurement for doped PBTTT implies the suppression of Hanle effect in highly doped organic semiconductors consistent with recent theoretical work [125]. In addition, charge transport properties of organic semiconductors such as the trap density was found to play a crucial role in spin current transmission in organic semiconductors where no spin signal can be observed for organic semiconductor with high trap density.

The vertical spin pumping measurement geometry can be also used to study interesting interfacial effect in organic-metal interfaces. Fig 5.6a shows the field dependence of the voltage for $\mathrm{Py} / \mathrm{Au}(6 \mathrm{~nm})$ and $\mathrm{Py} / \mathrm{PFBT} / \mathrm{Au}(6 \mathrm{~nm})$. PFBT is a self-assembled monolayer (SAM) molecule that can be functionalised on Au metal by immersing Au layer into a solution with PFBT and ethanol solution $(10 \mathrm{mMol})$ for 30 minutes. Clearly, the spin pumping signal increases when PFBT layer is inserted between Py and Au. This may be due to the recently proposed Rashba-Edelstein effect at SAM and metal interface (Fig 5.6b) where the charge transfer between the metal layer and SAM creates a 2D dipole layer with varying interfacial electric field depending on the charge transfer. A systematic study on the potential RashbaEdelstein effect at SAM and metal interface is obviously essential to understand the underlying physics. 

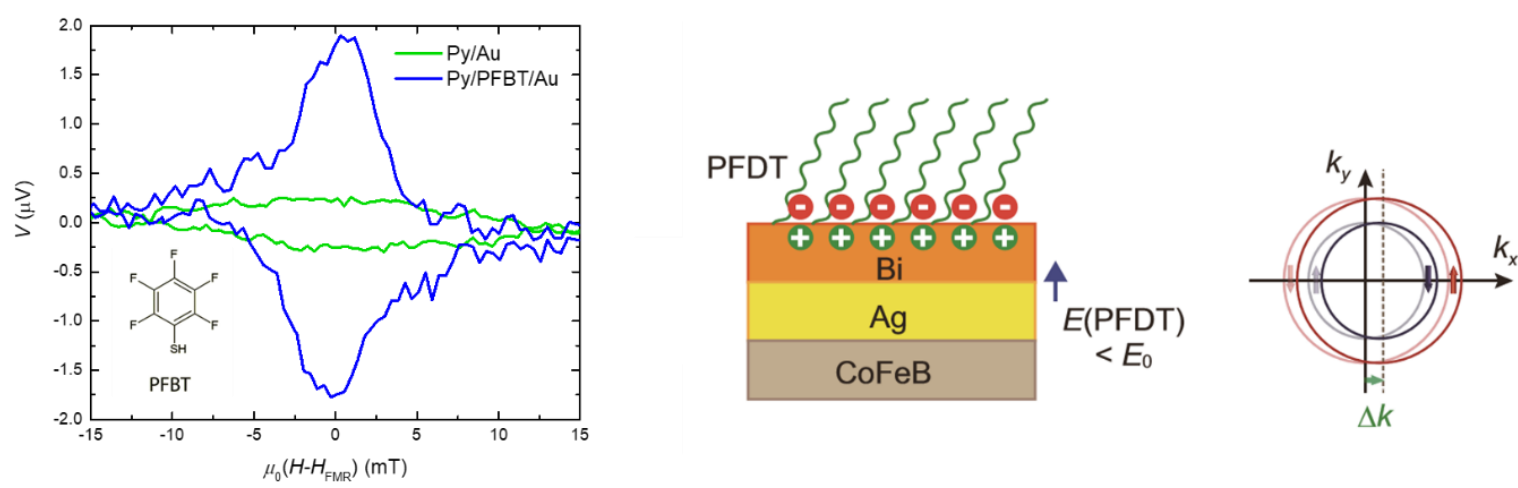

Figure 5.6 Molecular-metal Rashba spin to charge converter. a, Field dependence $\left(\mu_{0} H\right)$ of the electromotive force, $V$ measured for Py/Au $(6 \mathrm{~nm})$ (green line) and Py/PFBT/Au $(6 \mathrm{~nm})$ (blue line) at room temperature. The inset shows the chemical structure of 2,3,4,5,6-pentafluorothiophenol (PFBT). b, 2D dipole layer formed between PFDT molecule and Bi due to charge transfer. The Rashba-Edelstein effect is enhanced as a result of the charge transfer enhanced interfacial electric field as illustrated in the Fermi contour plot. Fig 5.6b is taken from [157]. 


\section{Chapter 6}

\section{Lateral spin transport in organic semiconductors probed by spin pumping and ISHE}

\subsection{Motivation}

As discussed in Chapter 4, the current spreading effect in electrical spin injection and detection device together with the stochastic noise generated by hopping conduction in organic semiconductors are identified as the intrinsic roadblocks to successfully probe spin transport in organic semiconductors using electrical spin injection techniques. Therefore, spin injection based on current-free technique potentially offers a solution to the intrinsic roadblocks. Spin pumping is a charge current-free spin injection technique based on dissipation of angular momentum via FMR. Coupling spin pumping with non-local spin detection technique is clearly a potential measurement architecture for probing spin transport in organic semiconductors. In this chapter, long range lateral spin transport was successfully observed in electrochemical doped organic semiconductors using a combination of spin pumping and ISHE in nanofabricated devices with spin diffusion lengths range from few hundred nanometres up to a micrometre. The experimental results are in quantitative agreement with theoretical work on spin transport mediated by exchange interaction in organic semiconductor systems using refined material specific parameters calculated from first principle*.

\footnotetext{
*The theoretical modelling results were calculated by INSPIRE group at Johannes Gutenberg Universität, Mainz. Note: Lateral spin pumping devices were fabricated together with Dr. Deepak Venkateshvaran.
} 


\subsection{Observation of lateral spin transport in organic semiconductors at room temperature}

To circumvent these roadblocks associated with electrical spin injection, transport, and detection in organic semiconductors using trilayer and nonlocal architectures, it becomes necessary to use experimental techniques that achieve spin injection without charge injection across an interface. One implementation of such a protocol is based on spin pumping involving the dissipation of angular momentum at a ferromagnetic injector and into a non-magnetic metal or semiconductor layer [158], [159], [62], [145], [146], [160]. Fig 6.1a shows a schematic of a lateral spin pumping architecture and measurement configuration that achieves this. Such a lateral spin pumping architecture was never previously demonstrated in organic semiconductors, but was successfully shown in p-Si [96], n-GaAs [100], Graphene [99], n-Ge [98], $\mathrm{Cu}$ [101], and a 2DEG [97]. The device consists of an island of a ferromagnetic permalloy (Py) layer fabricated next to a narrow, $1 \mu \mathrm{m}$ wide platinum (Pt) wire running parallel to an edge of the Py island, both of which are in contact with an organic semiconductor. The Py layer is driven into ferromagnetic resonance (FMR) by an applied microwave field in the presence of an external magnetic field and dissipates spin angular momentum into the adjacent organic semiconductor in the form of a spin current [11], [160], [159], [62], [87], [161]. This injected pure spin current propagates through the organic semiconductor and is absorbed in the Pt wire situated within a few hundred nanometres of the Py island. The absorbed spin current in the Pt detector is finally converted into an electromotive force via the inverse spin Hall effect (ISHE) in $\mathrm{Pt}$ and is measured as a voltage $V_{\text {ISHE }}$ across the platinum detector [11], [59], [56].

The conjugated polymer in our experiments is poly(2,5-bis(3-alkylthiophen-2-yl)thieno[3,2b]thiophene) (PBTTT) doped with 2,3,5,6-tetrafluoro-7,7,8,8-tetracyanoquinodimethane (F4TCNQ), a widely investigated model system. PBTTT is a semicrystalline conjugated polymer with a highly ordered lamellar microstructure, in which the polymer chains and the direction of $\pi-\pi$ stacking are oriented in the plane of the film and the aliphatic side chains approximately normal to the film plane giving rise to relatively high, in-plane charge carrier mobilities. The p-type molecular dopant, F4TCNQ, can be incorporated by a solid-state diffusion process or a sequential doping method that preserves the lamellar ordering and allows reproducible control of the carrier and spin concentration [162]. The dimensions of the device are illustrated in the optical image (Fig 6.1b) with the well resolved sub-micron gap $(300 \mathrm{~nm}$ in this case) between Py and Pt shown in the SEM image (Fig 6.1c). Initial measurements were 
performed inside an ESR cavity, where it was possible to measure the microwave absorption in Py and the ISHE voltage signal in Pt simultaneously. Fig 6.1d shows the microwave absorption signal of the $400 \times 600 \mu \mathrm{m}^{2}$ Py island on a complete device measured at $9.38 \mathrm{GHz}$ inside the electron spin resonance (ESR) cavity. Fig 6.1e is the corresponding voltage response measured across the Pt stripe when the Py island is brought into FMR within the ESR cavity. A clean ISHE signal is observed that peaks at the FMR resonance.

a

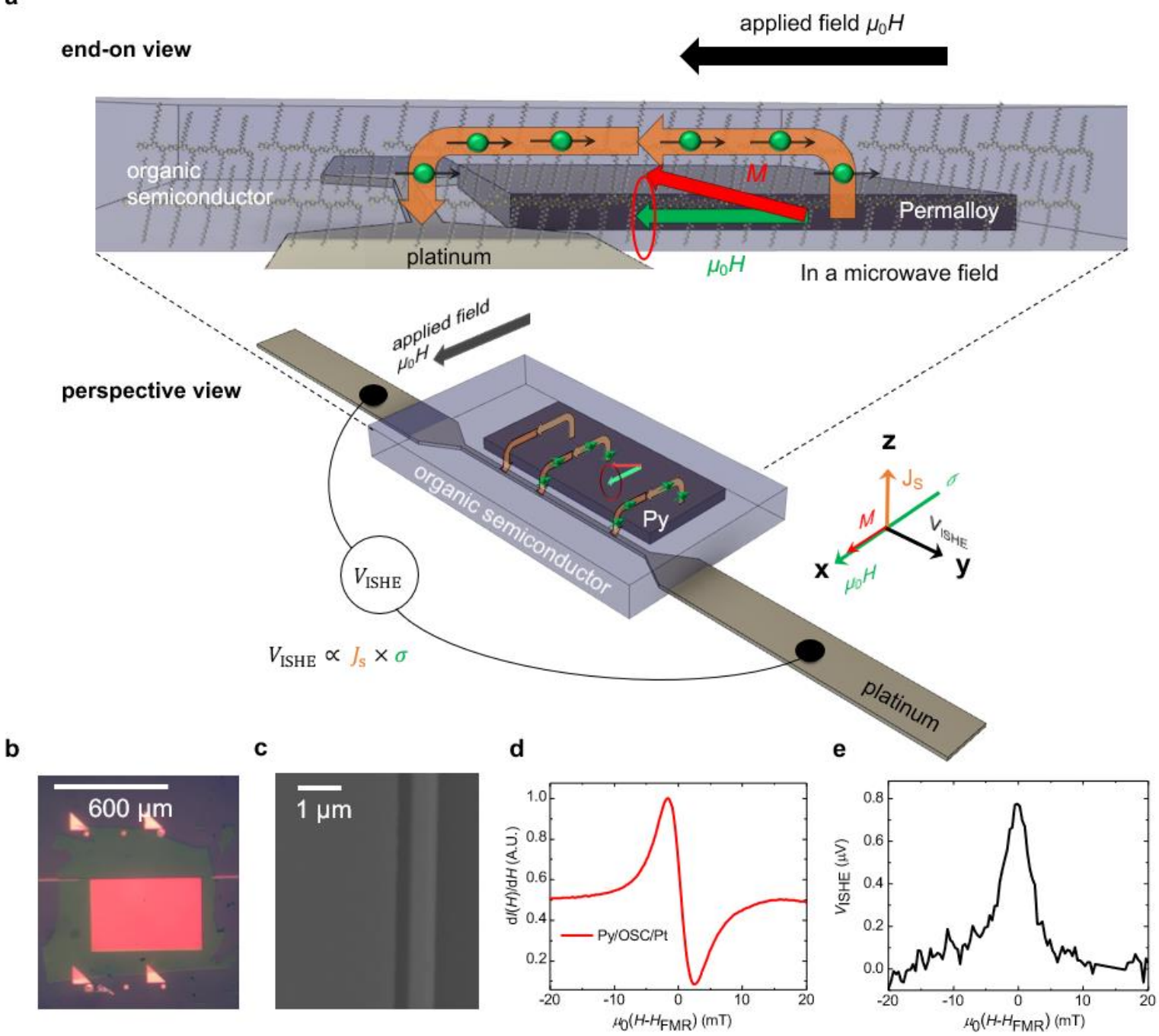

Figure 6.1 Lateral spin pumping device architecture and measurement scheme. a, Schematic illustration of lateral spin pumping and ISHE detection within the nanofabricated device. $\mu_{0} H, M, J_{\mathrm{s}}$ and $V_{\text {ISHE }}$ denote the external magnetic field, dynamic magnetisation, pure spin current and voltage due to ISHE, respectively. b, Optical image of the lateral spin pumping and ISHE device. $\mathbf{c}$, SEM image of $300 \mathrm{~nm}$ gap between Py and Pt electrodes. d, Field $\left(\mu_{0} H\right)$ dependence of FMR signal $d I(H) / d H$ measured for $\mathrm{Py} / \mathrm{OSC} / \mathrm{Pt}$ at $100 \mathrm{~mW}$ microwave excitation and $9.38 \mathrm{GHz}$ microwave frequency inside an ESR cavity. $I$ is the microwave absorption intensity. The external magnetic field was applied in a plane parallel to the film plane, but perpendicular to the long axis of the platinum detector. The FMR field is $100 \mathrm{mT}$. e, Voltage response picked up by the platinum stripe when the Py island is driven into FMR. 
Fig 6.2a shows the voltage signal detected with an incident microwave power of $1 \mathrm{~W}$ and a frequency of $4 \mathrm{GHz}$ for a device with a gap of $300 \mathrm{~nm}$ between Py and Pt. These measurements, as all subsequent ones, were performed with the sample placed on top of a microwave strip line that provided the FMR excitation. The signal is measured over a long Pt stripe (slightly longer than $600 \mu \mathrm{m}$ ) since the voltage signal scales linearly with length. The measured $V_{\text {ISHE }}$ signal was observed to invert in sign upon reversing the applied in-plane magnetic field direction, consistent with the characteristic symmetry of the inverse spin Hall effect, i.e., $E_{\mathrm{ISHE}} \propto J_{\mathrm{S}} \times \sigma$ [56]. Here, $E_{\mathrm{ISHE}}, J_{\mathrm{S}}$ and $\sigma$ denote the electric field generated by ISHE in the detector, flow of pure spin current, and spin polarisation vector of the spin current, respectively. The latter is aligned with the applied magnetic field. When $\sigma$ was polarised along the direction of the long axis of the Pt detector, $E_{\text {ISHE }}$ was found to vanish (Fig 6.7c). In a lateral spin pumping device, although there is an electrical contact between the ferromagnetic Py injector and the Pt detector through the doped organic semiconductor, the antisymmetric part of the measured signal due to the anomalous Hall effect (AHE) is reduced in comparison with vertical spin pumping devices on account of the lateral offset between the platinum detector and the permalloy injector.

To fully rule out the AHE contribution from the ferromagnetic layer, as well as potential artefacts due to the anomalous Nernst effect (ANE), and the Nernst effect (NE) of the doped organic semiconductor in the interpretation of the measured signal, a series of careful control experiments were performed. First, the Py island was capped with a $10 \mathrm{~nm} \mathrm{AlO}_{\mathrm{x}}$ layer to block spin injection into the organic semiconductor. As shown in the top sub-panel of Fig 6.2b, a spin signal at ferromagnetic resonance could not be measured in the presence of this blocking layer. Second, the platinum detector electrode was replaced with metals having a weaker spin-orbit coupling. When the detector electrode was replaced with $\mathrm{Cu}$, no observable signal was measured (Fig 6.2b bottom sub-panel), consistent with the very weak spin Hall angle of $\mathrm{Cu}$ [11]. When the detector electrode was replaced with $\mathrm{Au}$, the signal was strongly suppressed, though still detectable, (Fig 6.2c top sub-panel) as expected from the spin Hall angle of $\mathrm{Au}$ being significantly smaller than that of Pt [11]. Third, the platinum detector adjacent to the Py island was removed in the region next to the injector as shown in the bottom sub-panel of Fig 6.2c. A strong signal could not be observed in this configuration, convincingly ruling out spurious voltage contributions due to the ISHE and the Nernst effect of the doped organic semiconductor itself as contributions to our measured signal. These experimental results indicate successful spin transmission in the plane of the F4TCNQ-doped PBTTT film along 
the polymer backbone and the high mobility $\pi-\pi$ stacking direction. The normalised ISHE induced current, $I_{\text {norm }}=V_{\text {ISHE }} / R$, where $R$ is the measured resistance across the Pt wire, is observed to reduce exponentially with gap spacing between $\mathrm{Py}$ and $\mathrm{Pt}, L_{\mathrm{Py}-\mathrm{Pt}}$, as expected for spin decoherence with distance in the spin transport material (Fig 6.2d). The spin diffusion length, $\lambda_{s}$, for this sample of F4TCNQ doped PBTTT is estimated to be $1.2 \pm 0.1 \mu \mathrm{m}$ by fitting $I_{\text {norm }}\left(L_{\mathrm{Py}-\mathrm{Pt}}\right)=I_{0} e^{-L_{\mathrm{Py}-\mathrm{Pt}} / \lambda_{\mathrm{s}}}$ to the experimental data.

a

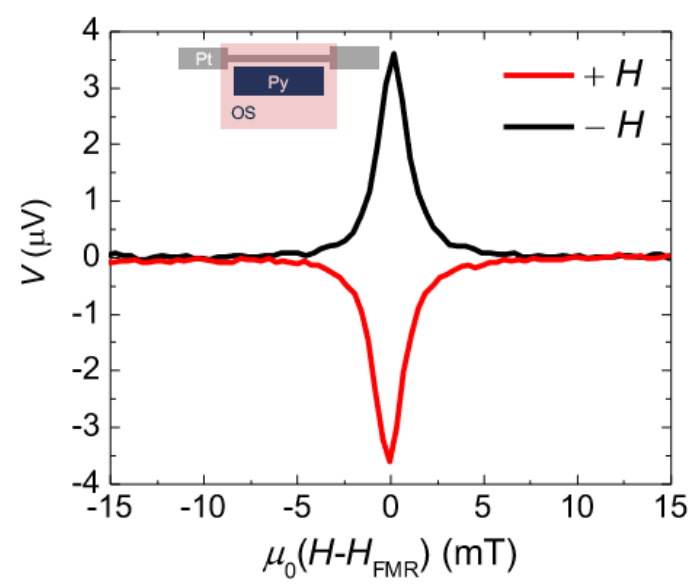

C

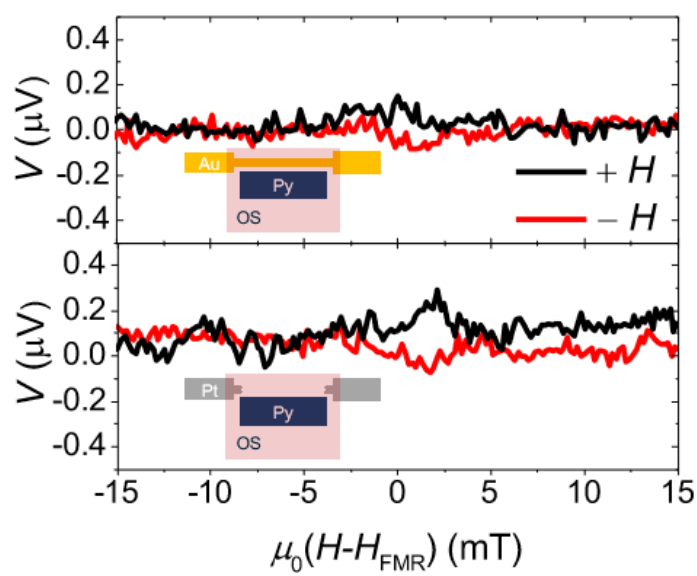

b
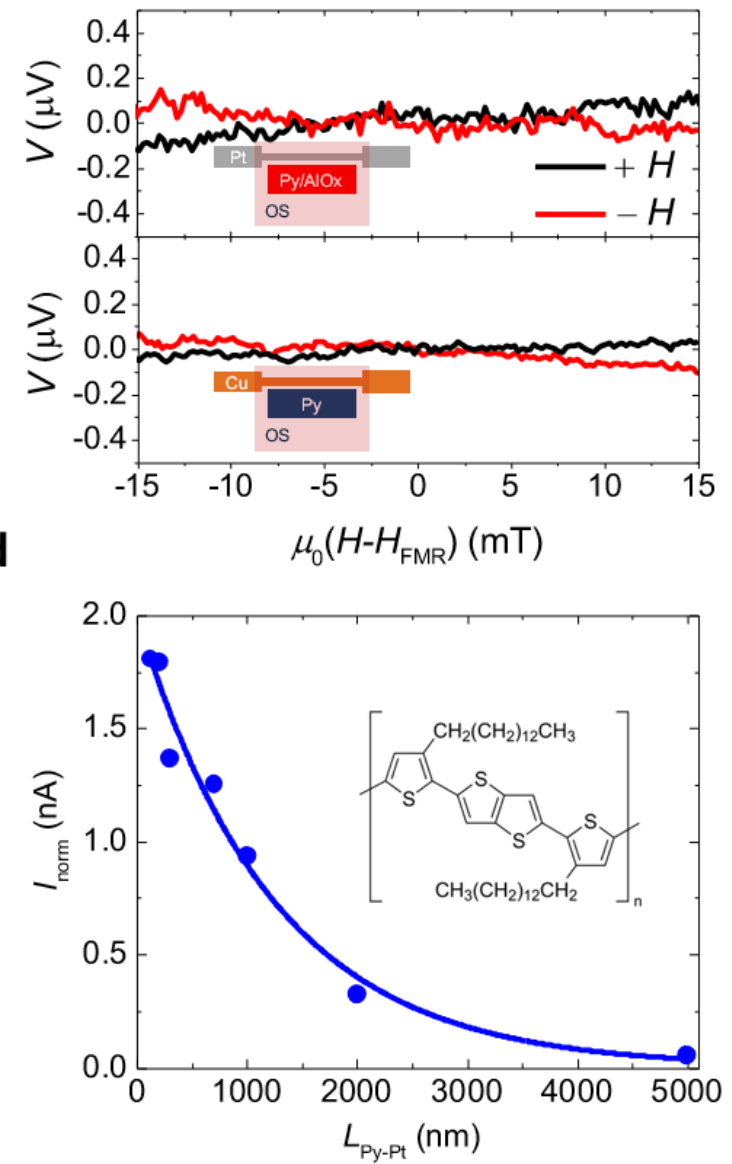

Figure 6.2 Observation of long range spin transport in F4TCNQ doped PBTTT. Field dependence of the electromotive force $V$ measured across the platinum detector for a, Py $(25 \mathrm{~nm}) / \mathrm{F} 4 \mathrm{TCNQ}-$ PBTTT/Pt (10 nm), b, top sub-panel Py $(25 \mathrm{~nm}) / \mathrm{AlO}_{\mathrm{x}}(10 \mathrm{~nm}) / \mathrm{F} 4 \mathrm{TCNQ}-\mathrm{PBTTT} / \mathrm{Pt}(10 \mathrm{~nm})$, bottom sub-panel Py $(25 \mathrm{~nm}) / \mathrm{F} 4 \mathrm{TCNQ}-\mathrm{PBTTT} / \mathrm{Cu}(10 \mathrm{~nm})$ c, top sub-panel Py $(25 \mathrm{~nm}) / \mathrm{F} 4 \mathrm{TCNQ}-$ PBTTT/Au (5 nm), bottom sub-panel Py (25 nm)/F4TCNQ-PBTTT/nano-fabricated broken Pt (10nm). The gap spacing between the Py island and platinum wire was $300 \mathrm{~nm}$ for all devices. The microwave power applied was $1 \mathrm{~W}$ (the microwave power absorbed by the device was around $160 \mu \mathrm{W}$ ) and the microwave frequency was $4 \mathrm{GHz}$. The FMR field ( $\left.H_{\mathrm{FMR}}\right)$ was close to $20 \mathrm{mT}$ under the given excitation conditions. d, Gap (between Py and Pt) dependence of ISHE induced current $I_{\text {norm }}=V_{\mathrm{ISHE}} / R$. The conductivity of the F4TCNQ-doped PBTTT film was around $100 \mathrm{~S} / \mathrm{cm}$. The blue sold line is an exponential fit. 
To better understand the nature of spin transport in F4TCNQ doped PBTTT, the spin signal measured at the platinum detector was studied as a function of carrier density in the organic semiconductor. The carrier density was varied by thermally annealing the fully doped, highly conductive film whereupon the dopant F4TCNQ molecules out-diffuse from PBTTT and the conductivity as well as carrier/spin concentration reduce [162]. Inorm was found to decrease very strongly when reducing the carrier concentration (Fig 6.3a). The extracted spin diffusion length within the film at various conductivities is shown in Fig $6.3 \mathrm{~b}$ and is clearly seen to decrease with conductivity, influenced by carrier density. Fig $6.3 \mathrm{c}$ shows the amplitude of the measured spin signal as a function of temperature in a lateral spin pumping device with a $300 \mathrm{~nm}$ channel and a fully doped PBTTT film. The spin signal at $50 \mathrm{~K}$ is a factor of three smaller than that at $300 \mathrm{~K}$. The conductivity over this temperature range reduces by a factor of four, accompanied by a reduction in both carrier density and mobility by a factor of approximately two each [162]. In comparison with the reduction in the spin signal strength due to de-doping (more than a factor of ten upon reduction of the conductivity by a factor of four), the reduction with temperature is weaker. This indicates that the drop in mobility, which occurs at low temperature, affects the signal less strongly than the reduction in carrier concentration and highlights the key role that the carrier/spin density plays in mediating spin transport within the polymer.

We have observed similar spin signals in other conjugated polymers. Fig $6.3 \mathrm{~d}$ shows a decaying spin signal with channel length between a Py injector and a Pt detector of a lateral spin pumping device with an active layer of F4TCNQ doped Poly(3-hexylthiophene-2,5-diyl) (P3HT). The spin diffusion length extracted for doped P3HT from this data was $635 \pm 174 \mathrm{~nm}$, several hundred $\mathrm{nm}$ shorter than that measured in PBTTT. At its maximum doping concentration, P3HT has a conductivity of only $3.2 \mathrm{~S} / \mathrm{cm}$ as opposed to $100 \mathrm{~S} / \mathrm{cm}$ in PBTTT. Despite this, P3HT shows a strong spin signal that reduces with conductivity (Fig 6.3e). It is instructive to note here that the spin density in doped films of PBTTT and P3HT measured using ESR are very similar, with PBTTT having a slightly lower value of $1.6 \times 10^{20} \mathrm{~cm}^{-3}$ in comparison to P3HT having $2.7 \times 10^{20} \mathrm{~cm}^{-3}$. In addition, it is known that on account of a higher crystalline ordering, PBTTT has a higher mobility than P3HT by up to an order of magnitude [163]. Thus, the observation, that both P3HT and PBTTT show a pronounced spin signal despite having a significant difference in their conductivities, points again to the key role that the mobile carrier density and the inter-polaron distance play in spin diffusion. Fig $6.3 \mathrm{f}$ shows a plot of the strength of the measured spin signal as a function of measured conductivity for PBTTT, P3HT and another doped conjugated polymer based on cyclopentadithiophene- 
benzothiadiazole (CDT-BTZ) for a fixed $L_{\mathrm{Py}-\mathrm{Pt}}=300 \mathrm{~nm}$ and provides further confirmation that spin information can be efficiently transported only in polymers with a large carrier density. Similar spin signals could not be measured in devices using undoped polymers.
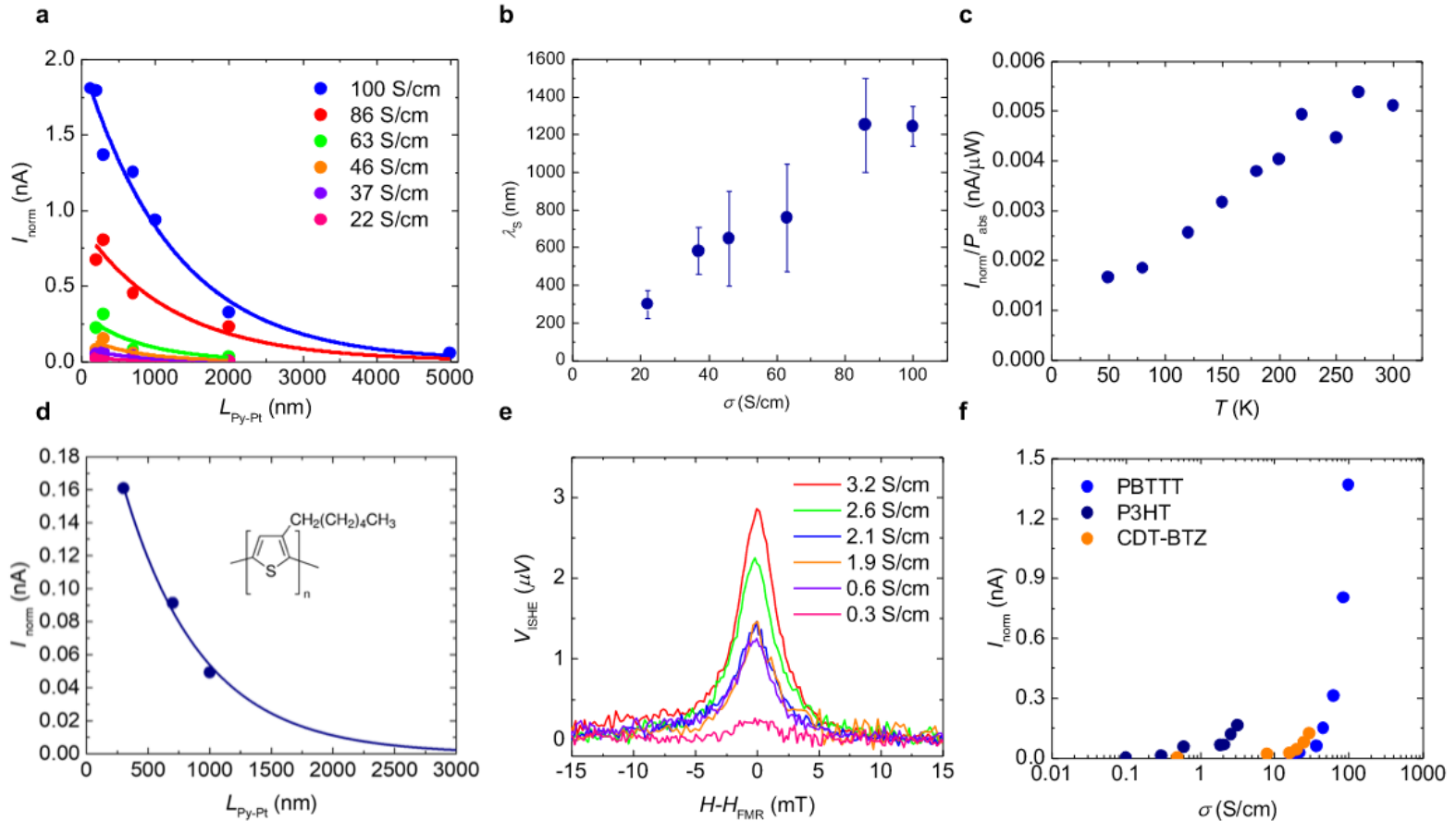

Figure 6.3 Carrier density dependence of spin current transport. a, Gap spacing between Py and Pt dependence of ISHE induced current with different conductivities of doped F4TCNQPBTTT film controlled by thermal annealing. The solid lines are exponential fits to experimental data. b, Conductivity dependence of spin diffusion length extracted from exponential fits. The error bars indicate the fit uncertainty. c, Temperature dependence of the spin signal in doped PBTTT. d, Spin diffusion length extracted from lateral spin pumping in P3HT. e, Conductivity dependence of the measured spin signal in P3HT. f, Plot of the measured spin signal as a function of conductivity of PBTTT in comparison with P3HT and CDT-BTZ.

\subsection{Out of plane angular, temperature and frequency dependence measurements}

We have also investigated the angular dependence of the voltage signal, when tilting the applied magnetic field out-of-plane (Fig 6.4a). As the applied magnetic field is rotated out-of-plane by an angle $\theta$, the orientation of magnetisation of the Py island lags by an angle $\theta-\phi$ owing to the shape anisotropy within the film. Using the Landau-Lifshitz-Gilbert (LLG) equation, the magnetisation angle $\phi$ is calculated from the $\theta$ dependence of the resonance field $\left(H_{\mathrm{FMR}}\right)$ shown in Fig 6.4b. The plot of $\phi(\theta)$ in Fig 6.4c demonstrates how the magnetisation of Py 
remains relatively in-plane up to $\theta \sim 60^{\circ}$, i.e. magnetisation and field are not collinear, but then reorients quickly as $\theta$ approaches $90^{\circ}$. From this we expect, first of all, the measured ISHE voltage to go through zero and change sign at $\theta=\phi=90^{\circ}$. This is cleanly observed in both PBTTT and P3HT (Fig 6.4d and 6.4e, respectively) and provides further validation of the interpretation of the experiment. The angular dependence also provides interesting information about how the orientation of the injected spin polarisation evolves while the spins diffuse through the polymer. Because the FMR-induced polarisation of the spins injected into the organic layer is along the magnetisation direction and the applied field is not collinear with the magnetisation, any reorientation of the spin polarisation due to Hanle-type precession around the applied magnetic field in the polymer, would change the x-polarised component of the spin current that reaches the Pt detector (Fig 6.1a) and manifest itself in an angular dependence that is significantly more gradual than the simple step-like, $V_{I S H E} \propto \cos (\phi)$ angular dependence that would be expected if the spin polarisation remained aligned with the magnetisation direction (Appendix B.II). In related tri-layer spin pumping experiments on undoped PBTTT [62], silicon [59], and pentacene [148], such a gradual angular dependence was claimed to have been observed, but not in $\mathrm{Alq}_{3}$ [145]. In our experiments, we observe a step-like angular dependence of $V_{\text {ISHE }}$ in both PBTTT and P3HT. Fig 6.4d shows the observed angle dependence of $V_{\text {ISHE }}$ in PBTTT for various device channel lengths, and a conductivity of $100 \mathrm{~S} / \mathrm{cm}$. It can be well explained by a simple $\cos (\phi)$ dependence (dashed line). The deviations seen in some of the samples are not systematic and are likely due to limited alignment accuracy in the experiments. Fig 6.4e shows a similar step-like angle dependence of $V_{\text {ISHE }}$ in P3HT for various conductivities but a fixed channel length of $L=700 \mathrm{~nm}$. This result suggests that during diffusion of the spins through the polymer the spin polarisation remains robustly aligned with the direction in which the injected spin current was polarised. 
b

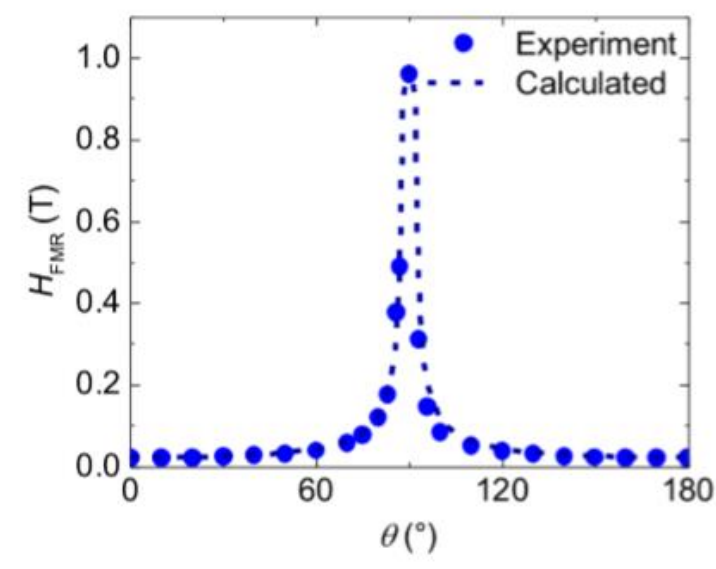

d

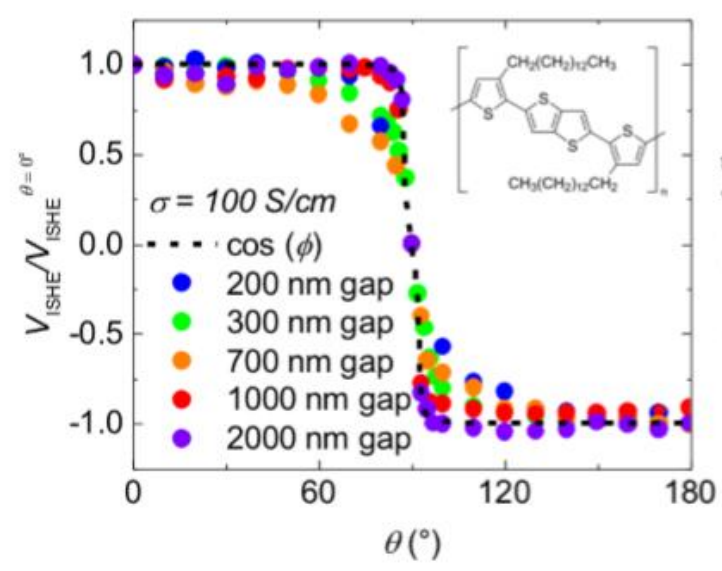

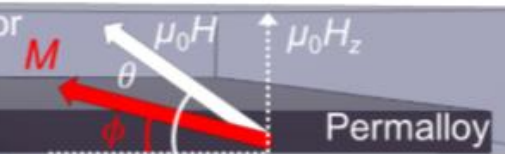

In a microwave field

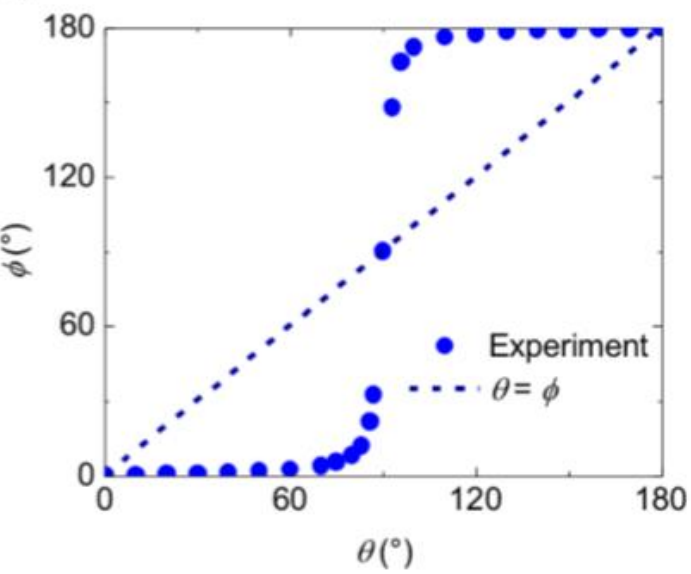

e

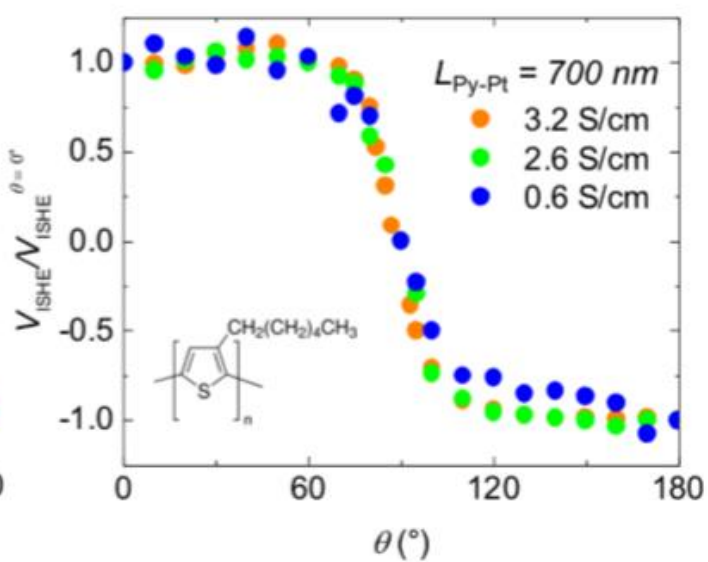

Figure 6.4 Angular dependence of the ISHE signal. a, Schematic geometry of Hanle measurements in lateral spin pumping devices. b, FMR resonance field at $4 \mathrm{GHz}$ as a function of the out-of-plane rotation of external magnetic field. c, Difference in angle between the ferromagnet's magnetization and the direction of applied field during rotation. d, Angle dependent measurements of $V_{\text {ISHE }}$ in PBTTT for various channel lengths. e, Angle dependent measurements of $V_{\text {ISHE }}$ in P3HT for various conductivities.

Fig 6.5 shows the $V_{\text {ISHE }}$ signal as a function of temperature for both magnetic field directions for $300 \mathrm{~nm}$ gap spacing lateral spin pumping device with doped PBTTT with F4TCNQ. The ISHE induced current normalised by device microwave absorption decreases with decreasing temperature (Fig 6.3c). The conductivity of F4TCNQ-PBTTT correspondingly reduces by a factor of four when the temperature is reduced from room temperature to $50 \mathrm{~K}$. At $50 \mathrm{~K}$, the 
conductivity of doped PBTTT is around $25 \mathrm{Scm}^{-1}$ and the measured ISHE signal normalised by the ISHE signal measured at $300 \mathrm{~K}\left(100 \mathrm{Scm}^{-1}\right)$ is 0.325 . For conductivity dependent measurements at room temperature, the ISHE signal measured at $22 \mathrm{Scm}^{-1}$ normalised by the ISHE signal measured at $100 \mathrm{Scm}^{-1}$ is only 0.018 . This indicates that the ISHE signal is affected more strongly by a reduction in conductivity that is caused mainly by a reduction in carrier concentration (as in the case of sample annealing leading to a loss of F4TCNQ dopant) than by similar reduction in conductivity caused at least partly by a reduction in carrier mobility (as is the case when cooling the samples to low temperature).

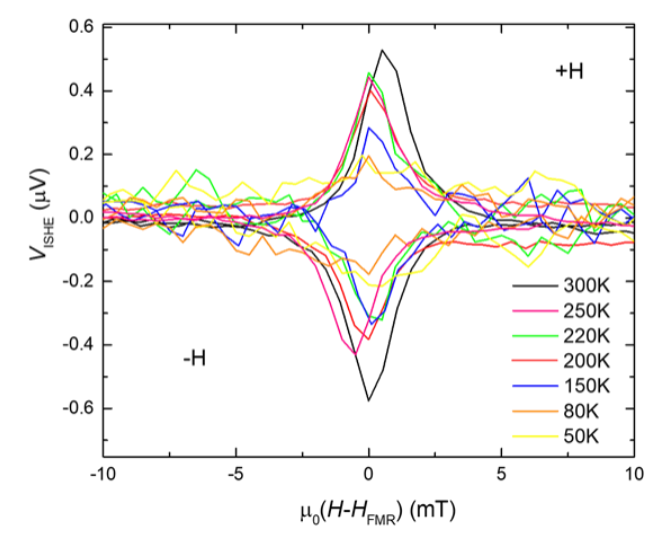

Figure 6.5 Temperature dependence of the ISHE signal. Temperature dependence of the ISHE voltage for both positive and negative in-plane magnetic field for lateral spin pumping device with 300 nm gap spacing. The spin transport material is doped PBTTT with F4TCNQ.

Microwave frequency dependence of the FMR of Py can be used to extract a few important parameters for gaining more insights in spin pumping processes. The frequency dependence of the resonance field, $H_{\mathrm{FMR}}$ can be fitted to the Kittel's formula $(\omega / \gamma)^{2}=H_{F M R}\left(H_{F M R}+4 \pi M_{S}\right)$ [86]. The fit provides an estimation of the saturation magnetisation, $4 \pi M_{S}=0.9535 \mathrm{~T}$ in good agreement with literature values. This indicates that the Py film is stoichiometric and is of high quality. The Gilbert damping constant is a parameter that quantifies the spin dissipation when a material is in contact with the ferromagnet. High spin-orbit coupling materials such as $\mathrm{Pt}$ cause a significant increase in the Gilbert damping constant when brought in contact with the ferromagnet since Pt dissipates spin effectively (spin sink). The Gilbert damping constant, $\alpha$ and the inhomogeneous broadening, $\Delta H_{0}$ can be extracted by fitting Eq. (6.1) to the frequency dependence of FMR linewidth. We observe a small enhancement of the Gilbert damping constant of Py after the deposition of doped organic semiconductors which could be a manifestation of efficient spin injection into the organic semiconductors. However, other factors that can influence linewidth broadening, such as potential modification of the surface 
of Py during the solution deposition of the conjugated polymer need to be considered carefully as well.

$$
\Delta H=\frac{4 \pi \alpha f}{\gamma}+\Delta H_{0}
$$

a

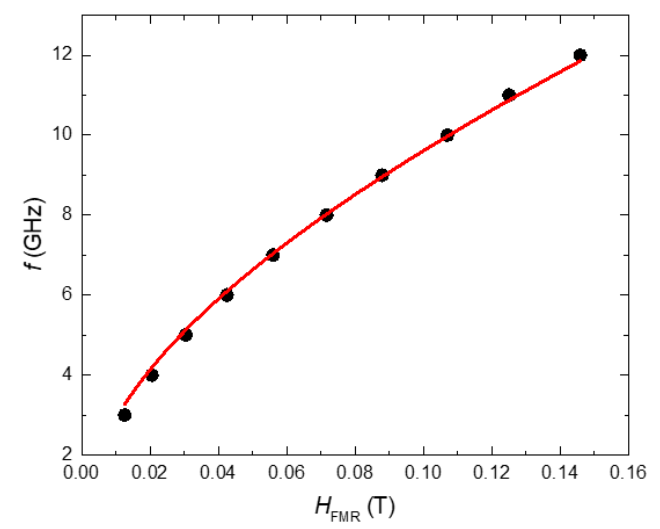

b

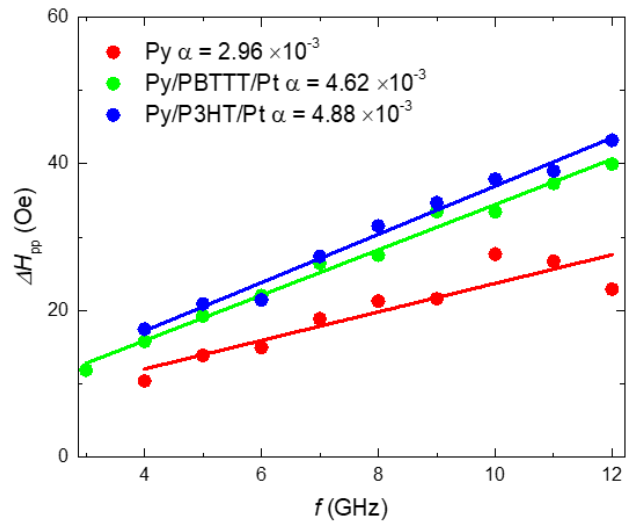

Figure 6.6 Frequency dependence of FMR of Py. a, Frequency dependence of the FMR field. The solid circles are the experimental data and the red curve is the fit based on Kittel's formula. b, Frequency dependence of the FMR linewidth. The solid circles are the experimental data and the solid lines are fit based on the equation above the figures. The legend shows the Gilbert damping constant $\alpha$ for different heterostructures.

\subsection{Electrical characterisation and additional control experiments}

A bias voltage was applied between the Py island and Pt wire to measure a current-voltage (IV) characteristic (Fig 6.7a). Before the deposition of doped PBTTT, there was no electrical connection between the Py island and Pt wire. This means the Py island does not overlap the Pt wire. An Ohmic IV characteristic is established between the Py island and Pt wire after the deposition of doped PBTTT.

For spin pumping, the density of spin current generated is proportional to the applied microwave power $P$. Thus, $V_{\text {ISHE }} \propto P$. The $V_{\text {ISHE }}$ signal increases linearly with microwave power (Fig 6.7b) which is consistent with the prediction of spin pumping theory. Fig 6.7c shows the field dependence of the voltage for Py-doped PBTTT-Pt (300 nm spacing) when the magnetic field is rotated $90^{\circ}$ in-plane. In this geometry, the Pt wire is parallel to the external magnetic field. No $V_{\text {ISHE }}$ signal can be measured in this configuration. This is consistent with the ISHE geometry $V_{\text {ISHE }} \propto J_{\mathrm{S}} \times \sigma$, where the spin polarisation of the spin current is defined 
by the external magnetic field direction.

Fig $6.7 \mathrm{~d}$ shows the field dependence of the voltage for the Py island when contact is made directly to it. The measurement geometry is identical to the lateral spin pumping and ISHE measurements. The signal is highly asymmetric and a fitting was performed to deconvolute the signal into symmetric and asymmetric parts. For the Py island, the symmetric part $V_{\text {sym }}$ was $2.431 \mu \mathrm{V}$ and the asymmetric part $V_{\text {asym }}$ was $1.945 \mu \mathrm{V}\left(V_{\text {sym }} / V_{\text {asym }}=1.25\right) . V_{\text {sym }}$ originates from the ISHE in Py and $V_{\text {asym }}$ comes from anomalous hall effect of Py. The $V_{\text {asym }}$ component can be used to quantify the spurious voltage contribution from Py to the $V_{\text {ISHE }}$ in lateral spin pumping and ISHE detection measurements. The $V_{\text {sym }} / V_{\text {asym }}$ ratio for the measured $V_{\text {ISHE }}$ signal for lateral spin pumping was 24.5 or greater which indicates that spurious signals in Py have minimal contribution to the measured signal. 
a

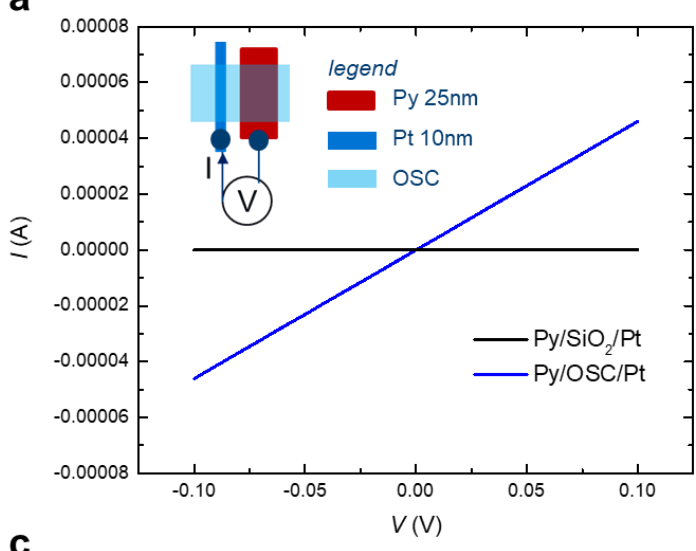

C

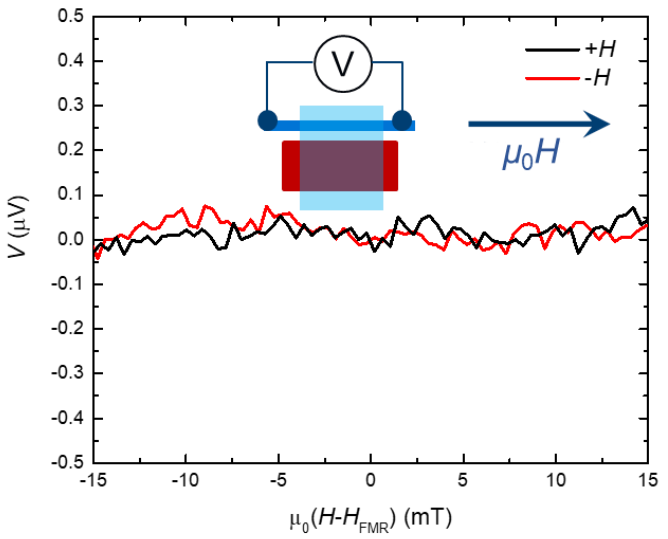

b

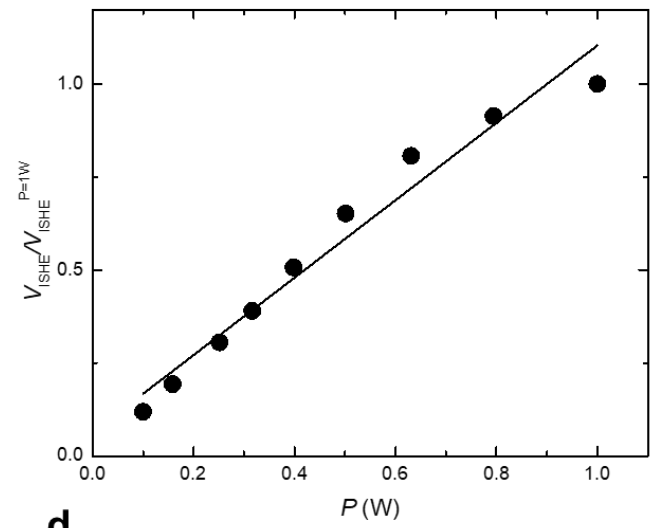

d

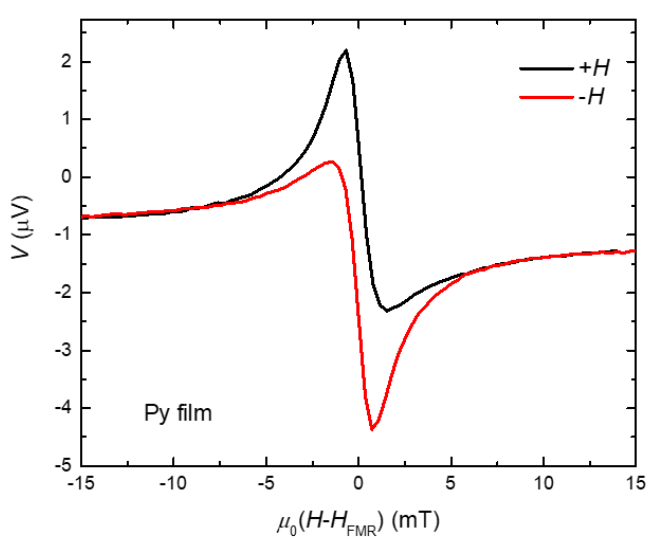

Figure 6.7 Electrical characterisation of the lateral spin pumping device and additional control experiments. a, IV characteristics of $\mathrm{Py}_{-} \mathrm{SiO}_{2}-\mathrm{Pt}$ (black) and Py-doped-PBTTT-Pt (blue). The gap spacing between Py and Pt is $300 \mathrm{~nm}$. The inset shows the measurement configuration. $\mathbf{b}$, Microwave power $P$ dependence of the ISHE voltage signal. The solid circles are experimental data and the solid line is a linear fit to the data. $\mathbf{c}$, in-plane field dependence of the measurement when the field is applied parallel to the platinum stripe, thus ensuring that the cross product in $V_{\text {ISHE }} \propto J_{\mathrm{S}} \times \sigma$ is zero. d, Field dependence of the electromotive force for Py island. The applied microwave power and frequency is 1 $\mathrm{W}$ and $4 \mathrm{GHz}$ respectively.

\subsection{Theoretical modelling of the results}

To explain the relatively long spin diffusion length $\left(\lambda_{s}\right)$ and the strong dependence of the ISHE voltage on carrier density we refer to a recently proposed theoretical framework based on exchange-mediated spin transport in organic semiconductors [127], [129], [132], [125]. The spin diffusion length is a function of the diffusion coefficient $D$ and the spin relaxation time $T_{1}$ through the relation $\lambda_{S}=\sqrt{D T_{1}}$. Within the exchange-mediated spin transport model, the diffusion coefficient $D$ is assumed to have additive contributions from charge/spin hopping $\left(D_{\text {hop }}\right)$ and also from exchange mediated coupling between spins on adjacent molecules $\left(D_{\text {exc }}\right)$, 
i.e., $D=D_{\text {hop }}+D_{\text {exc }}$. The latter can propagate spin information without requiring charge motion and is expected to be important only at sufficiently high carrier/spin concentration at which the typical inter-polaron/inter-spin distance $R$ approaches intermolecular distances but can lead to a large enhancement of the diffusion constant by several orders of magnitude. The total spin relaxation time $T_{1}$ includes contributions from spin relaxation due to local hyperfine interactions (HFI) and spin-orbit coupling (SOC) fields, i.e., $T_{1}=\left(\omega_{H F I}+\omega_{S O C}\right)^{-1}$. The hopping contribution to the diffusion coefficient depends on the carrier mobility $\mu$ through the Einstein relationship, $D_{h o p}=\mu k_{B} T / e$. We use here a one-dimensional (1D) formulation of the originally $3 \mathrm{D}$ model because of the specific microstructure of PBTTT, in which polarons are most likely to come close to each other along the $\pi-\pi$ stacking direction, i.e., we have made explicit modifications to the original 3D expressions for $D_{\text {exc }}$ and $\tau$ used in (Appendix A.I) [125]. The exchange contribution to the diffusion coefficient bears the form for an isotropic Heisenberg chain in $1 \mathrm{D}, D_{\text {exc }}=1.6 J(R) R^{2}$, where $J(R)$ is the exchange interaction [164] and may be approximated by a spatially isotropic, hydrogenic exchange interaction of the form $J(R)=0.821\left(e^{2} / \varepsilon \xi\right)(R / \xi)^{5 / 2} e^{-2 R / \xi}$ as a function of the polaron localisation length $\xi$ and $R$ [125], which gains in strength as the distance between the carriers is reduced. We have computed the polaron localization length along the $\pi-\pi$ stacking direction to be $\xi=0.4 \mathrm{~nm}$ for PBTTT from first principles. The spin relaxation rate due to the local HFI [125], [126] is $\omega_{H F I}=2 \Omega_{H F I}^{2} \tau / 3$ where $\Omega_{H F I}$ is the Larmor frequency of the local hyperfine magnetic field and the time $\tau=\left(2 D_{\text {hop }} / a^{2}+2 D_{\text {exc }} / R^{2}\right)^{-1}$ is a measure of the dwell time of a spin on a molecule as it propagates in $1 \mathrm{D}$ along the $\pi-\pi$ stacking direction. $a=0.45 \mathrm{~nm}$ is the average hopping distance between polarons along the $\pi-\pi$ stacking direction. The spin relaxation rate due to SOC is $\omega_{S O C}=2 \chi^{2} \tau^{-1}$ where $\chi^{2}=(4 / 3) \gamma^{2}$ is the spin-mixing parameter due to the SOC and $\gamma^{2}$ is calculated from first principles.

Within this model a long spin diffusion length is explained in terms of a large exchange dominated diffusion coefficient. Because the spatial decay length of the exchange interaction is short, a small separation between polarons/spins is necessary for this mechanism to become prominent, i.e., a path of occupied electronic states separated by sufficiently short distances is a prerequisite. This scenario is achieved at large doping concentrations (large $n$ and small $R$, where $R=1 / \sqrt[3]{n}$ ). A second requirement for a long spin diffusion length is that the $T_{1}$ is sufficiently long. Density-functional theory calculations of the hyperfine field strength in the polymer units revealed weak fields of $<10$ gauss, indicating a dominance of the SOC spin 
relaxation mechanism over HFI (Appendix A.III). The electronic states in a semiconductor are a mix of spin up and spin down states on account of which an electron hopping through a polymer network loses spin polarization through the Elliott-Yafet (EY) mechanism [106], [107]. A long spin relaxation time $T_{1}$ therefore requires a weak SOC, in particular a small value of $\gamma^{2}$.

Although the polymers chosen in this study have relatively large mobilities between 0.1 (for P3HT) and $1 \mathrm{~cm}^{2} / \mathrm{Vs}$ (for PBTTT), at large carrier concentrations the exchange diffusion coefficient $D_{\text {exc }}$ dominates the overall diffusion coefficient $D$ by two orders of magnitude as shown in Fig 6.8a. In the model both $n$ and $\gamma^{2}$ impact the spin relaxation time $T_{1}$, while $n$ impacts $D$ through the exchange integral $J(R)$. Fig $6.8 \mathrm{~b}$ shows the calculated spin diffusion length $\lambda_{S}$ plotted as a function of carrier density $n$ under the assumption of constant carrier mobility. In the regime where the diffusion constant is dominated by the exchange mechanism, the theoretically predicted spin diffusion length achieves values of over $1 \mu \mathrm{m}$. This prediction is matched by the experimentally determined values of $\lambda_{S}$ shown in Fig 6.8b. The experimental value of the carrier density for the highest conductivity in PBTTT and P3HT were measured using ESR and taken together with the measured conductivity of $100 \mathrm{~S} / \mathrm{cm}$ for PBTTT, the mobility was extracted to be $\sim 4 \mathrm{~cm}^{2} / \mathrm{Vs}$ using $\sigma=n e \mu$. This carrier mobility, although high, is not unwarranted seeing as the distance between the injector and the detector is a few hundred nanometres, thus potentially reflecting intra-grain transport. The carrier densities of the other measured data points shown in Fig $6.8 \mathrm{~b}$ were estimated from the measured conductivities that depend on doping concentration using the mobility determined by ESR at the highest carrier density. The experimental data of Fig $6.8 \mathrm{~b}$ clearly tracks the decline in the spin diffusion length as the exchange interaction is weakened due to increasing distances between polarons/spins on de-doping the film, providing strong evidence for exchange-mediated spin diffusion being the mechanism responsible for the long spin diffusion length observed here. We also find that the predicted exchange diffusion coefficient is consistent with the measured $\lambda_{S}$ and the value of $T_{1}$ determined independently by ESR (Appendix B.III) according to $\lambda_{S}=\sqrt{D T_{1}}$.

To explain the difference in spin diffusion length between P3HT and PBTTT, which have similar carrier densities and $\pi-\pi$ stacking distances, we consider differences in the SOC strength [127], [128]. The parameter $\gamma^{2}$ in a conjugated molecule increases when the adjacent units are not coplanar but exhibit finite torsion angles between each other [128]. In PBTTT, we expect maximal spin diffusion lengths to be determined by virtually flat polymer chains, 
correspondingly to a low $\gamma^{2}$. In P3HT, literature suggests a variation of thiophene - thiophene dihedral angles between 20 - 30 degrees, and 40 - 60 degrees in comparable polymer samples [165], [163], [166]. We consequentially calculate $\gamma^{2}$ for representative geometries in these two limits. Fig 6.8c shows the joint dependence of the spin diffusion length $\lambda_{\mathrm{S}}$ on $n$ and on the updown spin admixture parameter $\gamma^{2}$ that reflects the strength of SOC. $\lambda_{\mathrm{S}}$ is nearly inversely proportional to $\gamma$, and increases with increasing $n$.

At this point, we emphasize that the accuracy of our theoretical spin diffusion length predictions far exceeds reasonable expectations on the model used. While all model parameters have been taken either directly from experiments or calculated from electronic structure theory and adapted to (effectively) 1D transport corresponding to our understanding of the actual spin dynamics in the polymer samples, our predictions sensitively depend on the balance between parameters, as well as between parameters and the model itself. The simplicity of the model therefore suggests errors cancelling to a significant degree. In particular, test calculations indicate that charge motion at intermediate polaron concentrations may significantly contribute to spin diffusion. This effect is neglected in a static spin diffusion model, therefore underestimating the predicted spin diffusion lengths. On the other hand, the exchange coupling expression derived in - [125] even with a polaron delocalization length estimated from firstprinciples as used here - is significantly stronger than frequently used expressions [167] at precisely the same intermediate spin concentrations, which appears to cancel the error due to lack of dynamic effects.

Finally, we would like to discuss possible reasons why the spin polarization remains robustly aligned with the magnetization direction and does not appear to precess around the external magnetic field, as the spins diffuse through the organic layer over micrometer distances. One possible interpretation of this result is that the spins transit through the polymer on a timescale $\tau_{T}$ that is faster than the timescale $\tau_{L}$ for precession in the external magnetic field. It is tempting to interpret this as a characteristic feature of rapid exchange-enhanced spin diffusion as it was in fact claimed theoretically [125] to explain the absence of a Hanle signature in vertical organic spin valves [40], [168]. In our system, the exchange-enhanced spin transit time $\tau_{T} \approx L^{2} / D_{\text {exc }}$ is indeed very fast, on the order of $10 \mathrm{~ns}$. However, it remains comparable to $\tau_{L} \approx 2 \pi / \gamma_{e} B_{\text {ext }}^{\perp}$, where $\gamma_{e}$ is the electron gyromagnetic ratio and $B_{e x t}^{\perp}$ is the component of the external field perpendicular to the magnetization. Using a simplified model of the experiment discussed in 
Appendix B.II, we argue that spin precession should have manifested itself in a more gradual angular dependence than what is observed experimentally, unless $D_{\text {exc }}$ was in fact even higher than predicted by the exchange-based spin diffusion model. This seems unlikely because the relationship $\lambda_{S}=\sqrt{D T_{1}}$ puts a bound on $D_{\text {exc }}$ values to be compatible with the measured values of $\lambda_{S}$ and $T_{1}$. A second, more likely, interpretation is that it is in fact an inherent feature of the lateral spin pumping architecture. It is worth noting that also in very carefully performed experiments on Si and Graphene, where it has been possible to perform both lateral spin pumping and non-local electrical spin injection and detection measurements and establish that both experiments yield consistent and reliable values for the spin diffusion length, a Hanle signature was observed only in the nonlocal spin valve but not in the lateral spin pumping architecture [96], [99]. In fact, none of the lateral spin pumping experiments reported to date have shown a gradual angular dependence (Table 2.2). In the simplified model of Appendix B.II we have neglected the fact that in the lateral spin pumping experiment the injected spin polarization has not just a DC component but also an AC component, which is driven by the $\mathrm{AC}$ microwave field and is precessing around the magnetization direction. It is possible that in the rotating frame of reference of the precessing spins a DC applied magnetic field is very ineffective in re-orientating the axis of this spin precession and that the axis of precession is kept aligned by the AC microwave field. In fact, theoretical considerations have argued that the driving AC microwave can even cause a spin rectification effect that enhances the DC component of the spin polarization along the magnetization direction [169]. To observe a signature of Hanle precession in the lateral spin pumping architecture may require more challenging $\mathrm{AC}$ measurements, which go beyond the scope of the present work, whose focus is on explaining the exceptionally long spin diffusion lengths observed in doped conjugated polymers. 
a

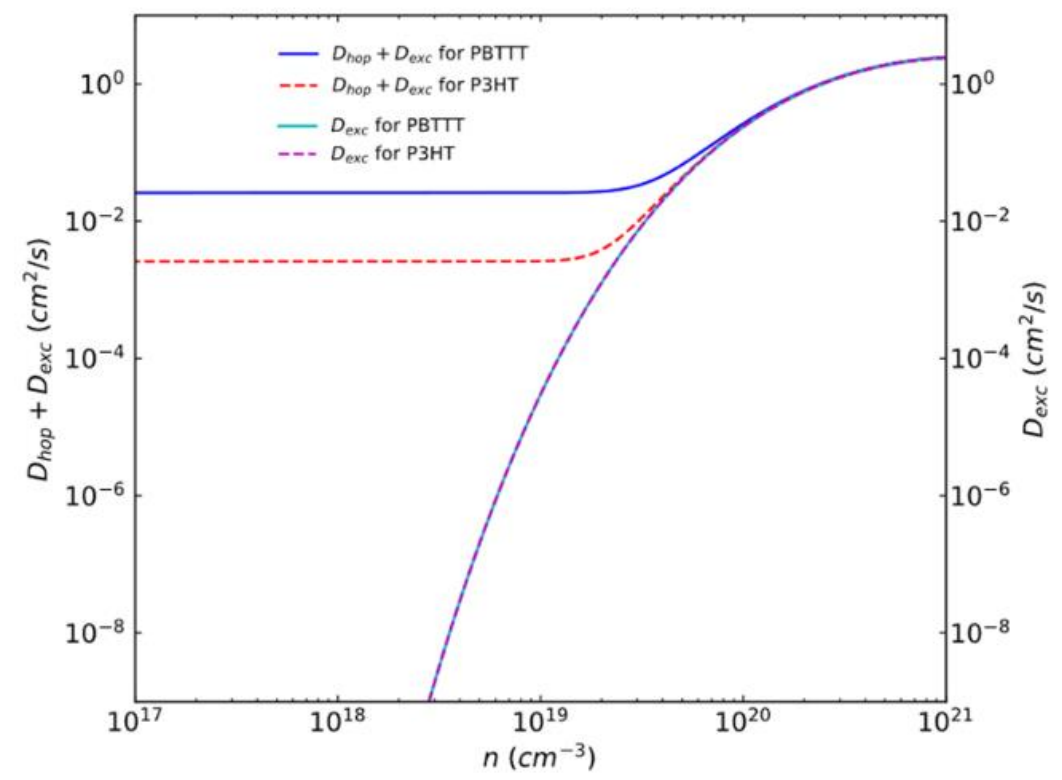

b

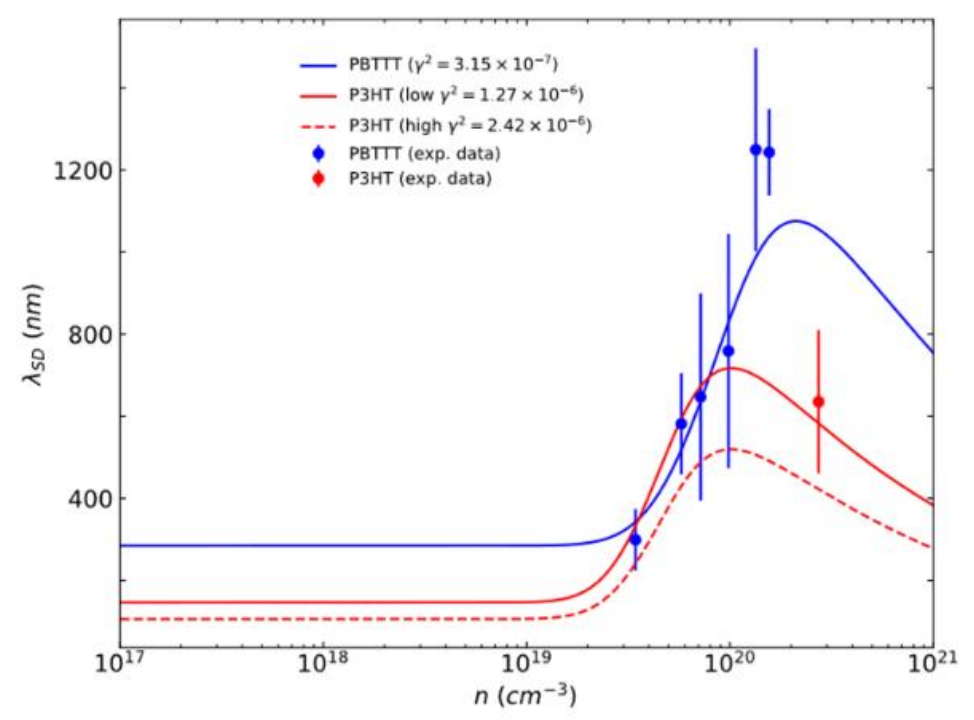

C

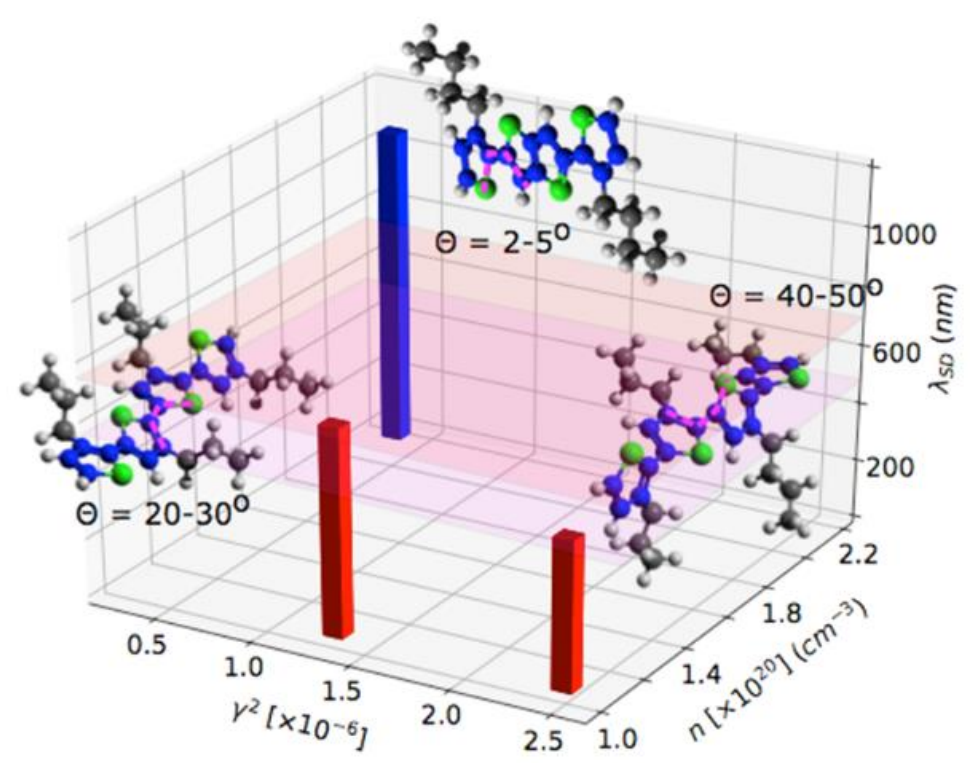


Figure 6.8 Theoretical modelling of spin transport in an exchange mediated regime. a, Total diffusion coefficient that includes contributions from hopping and exchange as a function of carrier density for PBTTT and P3HT. b, Spin diffusion length as a function of carrier density for PBTTT and P3HT. The maximum spin diffusion length for PBTTT is $1.102 \mu \mathrm{m}$ at a theoretically predicted $n=1.91 \times 10^{20} \mathrm{~cm}^{-3}$. For P3HT with low value of spin-mixing parameter $\gamma^{2}=1.27 \times 10^{-6}$, it is $0.72 \mu \mathrm{m}$ and with a high value of $\gamma^{2}=2.42 \times 10^{-6}$, it is $0.52 \mu \mathrm{m}$ at a theoretically predicted $n=1.01 \times 10^{20} \mathrm{~cm}^{-3}$. c, The spin diffusion length for PBTTT and P3HT shown as a function of both $\gamma^{2}$ and $n$. A variation in $\gamma^{2}$ is caused by different dihedral angles in the molecule.

\subsection{Conclusions}

In order to overcome the intrinsic roadblocks presented in the electrical spin injection measurement scheme, spin pumping, a charge-free spin injection technique together with ISHE spin detection method was used to measure lateral spin transport in organic semiconductors using a nanofabricated device architecture. This approach successfully overcomes both the conductivity mismatch problem and the measurement noise caused by current spreading and hopping transport and enables spin transport in organic semiconductors to be probed electrically.

Long range lateral spin transport of up to a micrometer in doped conjugated organic semiconductors have been measured experimentally. The necessity to enter a regime of high carrier density to access the spin transport mechanism based on exchange coupling between polarons that enables efficient spin transport was demonstrated. The experimental results are in good agreement with the results modelled theoretically based on spin transport mediated by exchange interaction between polarons model and materials specific parameters obtained from first principle. This finding opens up new avenues to tune the chemical and electrical properties of organic semiconductors to turn them into useful and highly effective spintronic materials. 


\section{Chapter 7}

\section{Conclusions and Outlook}

This thesis explored the spin transport in organic semiconductors using a variety of experimental techniques from all electrical spin injection and detection to ferromagnetic resonance spin pumping and ISHE spin detection. This chapter summarises the main conclusions of this dissertation and discusses potential future research directions and technological applications.

Despite diligent attempts to study non-local spin transport in organic semiconductors using a number of novel electrical spin injection and detection techniques from conventional four terminal organic spin valve to electrical spin injection and ISHE spin detection method, no convincing spin signal can be observed in these device architectures. The intrinsic roadblocks for electrical spin injection-based measurements were identified as the current spreading effect and the hopping conduction in organic semiconductors which makes all electrical non-local spin injection and detection measurements extremely challenging if not impossible for organic semiconductors.

The out of plane spin transport in a range of organic semiconductors was studied by tri-layer spin pumping architecture. The spin current transmission in organic semiconductors depends strongly on the molecular structure and the charge transport properties of the polymers. The spin diffusion length for PBTTT was found to increase with the carrier concentration where the carrier density is controlled by electrochemical doping with F4TCNQ. Moreover, no spin signal can be observed for organic conjugated polymer with high trap density. As a side project, deposition of heavy metal layer on top of organic semiconductor without damaging the organic semiconductor layer was optimised by tuning the magnetron sputter deposition parameters. The optimised deposition conditions can be used to deposit heavy metal electrodes on top of organic polymers for a number of organic spintronics applications such as optical spin injection and ISHE detection measurements. 
Lateral spin diffusion length of up to a micrometre was observed in doped conjugated polymers using a microfabricated device architecture where spin current is injected by spin pumping and detected by ISHE. The experimental results are supported by theoretical calculations based on exchange mediated spin diffusion model and parameters obtained from first principle. This non-local spin transport device architecture provides a platform for studying spin transport in a wide range of organic semiconductors where the spin current propagates along the high mobility direction.

There are several future research questions and challenges waiting to be solved. First, no "Hanle" type angular dependence was observed in lateral spin pumping experiments based on electrochemically doped conjugated polymers. This might arise due to suppression of Hanle effect in these systems where the spin transport is mediated by exchange coupling between polarons at high enough carrier (spin) concentration. Clearly, it would be interesting to study the angular dependence of spin pumping signal in organic semiconductors (small molecules single crystal and conjugated polymer) with very high mobility exceeding $10 \mathrm{~cm}^{2} \mathrm{~V}^{-1} \mathrm{~s}^{-1}[170]$, [171], [172], [173], [174] at relatively low carrier concentration where exchange interaction between polarons is expected to be negligible. Preliminary lateral spin pumping measurements with high mobility donor-acceptor organic semiconductor $\left(3.3 \mathrm{~cm}^{2} \mathrm{~V}^{-1} \mathrm{~s}^{-1}\right.$ OFET mobility), CDT-BTZ show a more "gradual" out of plane angular dependence at low carrier concentration (Fig 7.1b). Even though this may be an indication of the Hanle effect, more measurements are required to fully understand and confirm the angular dependence measurements. 
a

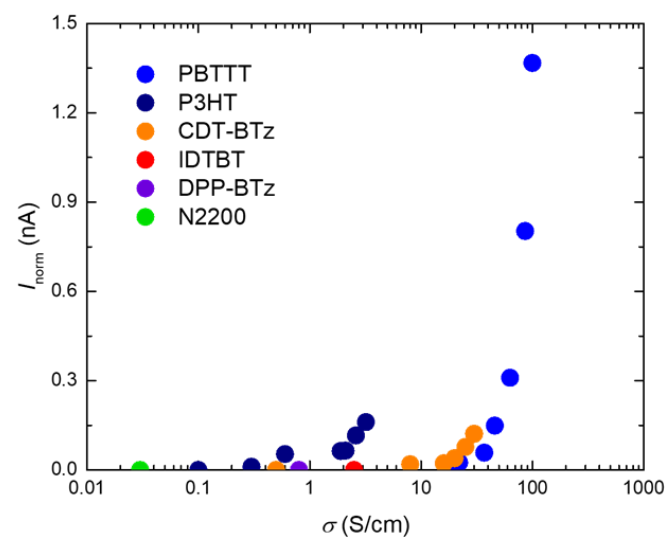

b

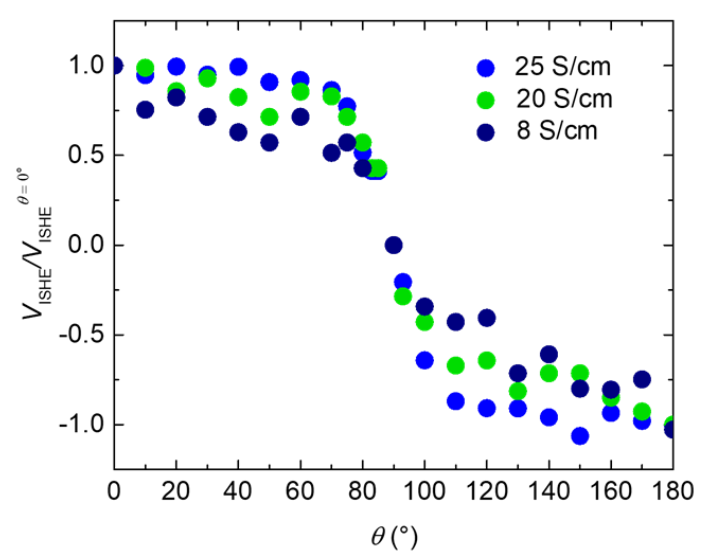

Figure 7.1 Lateral spin pumping and ISHE detection for different materials at room temperature.

a, Plot of the measured spin signals as a function of conductivity of various polymers. $\mathbf{b}, V_{\mathrm{ISHE}}$ measured as a function of out of plane magnetic field angle, $\theta$ for F4TCNQ doped CDT-BTZ with different conductivity values.

Second, for practical applications, it is necessary to modulate the spin signal with external stimuli for high performance computing applications [78], [77], [175], [176]. The initial proposal of spin transistor concept was based on a $2 \mathrm{DEG}$ lateral spin valve where a gate voltage can be applied to modulate the spin valve signal [177]. Extending from this concept, the lateral spin pumping device architecture can be modified slightly to enable external control. In particular, a gate electrode together with a layer of dielectric material can be placed on top or bottom of the spin transport channel analogous to conventional organic field-effect transistor. Based on the exchange mediated spin transport framework, in this geometry, the spin transport channel can be switched on and off by an externally applied gate voltage where the spin channel is switched on when a gate voltage is applied to induce charge accumulation (exchange interaction switched on) and vice versa. Preliminary gated lateral spin pumping measurement based on Rubrene single crystal (organic small molecules with very high mobility of greater than $10 \mathrm{~cm}^{2} \mathrm{~V}^{-1} \mathrm{~s}^{-1}$ ) was attempted. However, no spin signal can be observed both with and without the gate voltage of $-60 \mathrm{~V}$ where the gate voltage is applied via the silicon substrate bottom gate and the $\mathrm{SiO}_{2}$ on top of silicon substrate acts as the dielectric layer (Fig 7.2a). There could be a number of reasons as to why this measurement didn't showed a spin signal, first, the Rubrene single crystal is not freshly made and the mobility value has dropped to around 0.1-0.2 $\mathrm{cm}^{2} \mathrm{~V}^{-1} \mathrm{~s}^{-1}$ as confirmed from OFET measurements due to degradation over time. Second, the transfer curve of rubrene single crystal measured using the lateral spin pumping device showed significant hysteresis which may be caused by the instability of Rubrene single 
crystal in ambient conditions such as moisture in air. Finally, there are some air gaps formed near the active Py-Pt region during the lamination of Rubrene single crystal on the lateral spin pumping device which could reduce the spin signal. Therefore, this measurement requires some optimisations for obtaining reliable results. It is important to note that a low leakage current is required for this measurement to work since high leakage current will translate into high noise in the voltage measurement across the Pt detector as confirmed by lateral spin pumping measurement with ion-gel gating where the leakage current is high.

a

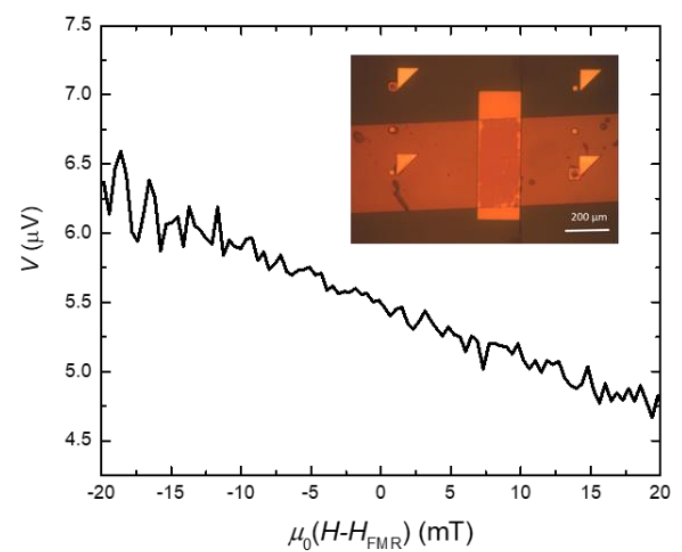

b

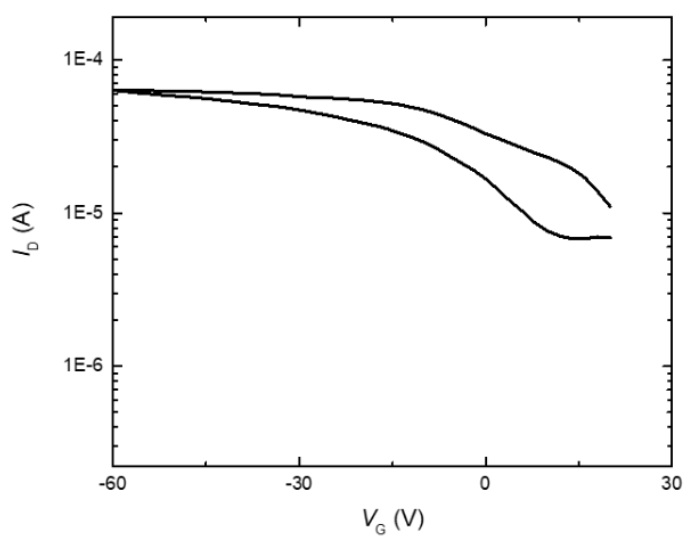

Figure 7.2 Lateral spin pumping and ISHE detection for Rubrene single crystal device at room temperature. a, Field dependence of the electromotive force, $V$ measured across the platinum detector for Rubrene single crystal device. The gap spacing between Py and Pt is $300 \mathrm{~nm}$ and the gate voltage of $-60 \mathrm{~V}$ is applied via the silicon substrate bottom gate. The microwave power applied is $1 \mathrm{~W}$ and microwave frequency is $4 \mathrm{GHz}$. The inset shows an optical image of the lateral spin pumping device with Rubrene single crystal laminated. b, Transfer curve for Rubrene single crystal measured on the lateral spin pumping device (drain voltage, $V_{\mathrm{d}}=-60 \mathrm{~V}$ ) where the silicon substrate is the bottom gate and the $\mathrm{SiO}_{2}$ is the dielectric layer.

Finally, to complete the full circle of the lateral spin transport in organic semiconductors exploration, a non-local spin valve measurement operated by spin pumping can be attempted with organic semiconductor spin channel. In this measurement, spin injection is achieved via spin pumping but spin accumulation is used as the detection method. This will not only be an additional experimental approach to study spin transport in organic semiconductors but can also provide further insights to the spin injection and transport physics in organic semiconductors using well established non-local spin valve theory. This approach has successfully demonstrated spin transport in all metallic lateral spin valve with silver spin transport channel as shown in Fig 7.3. 
a

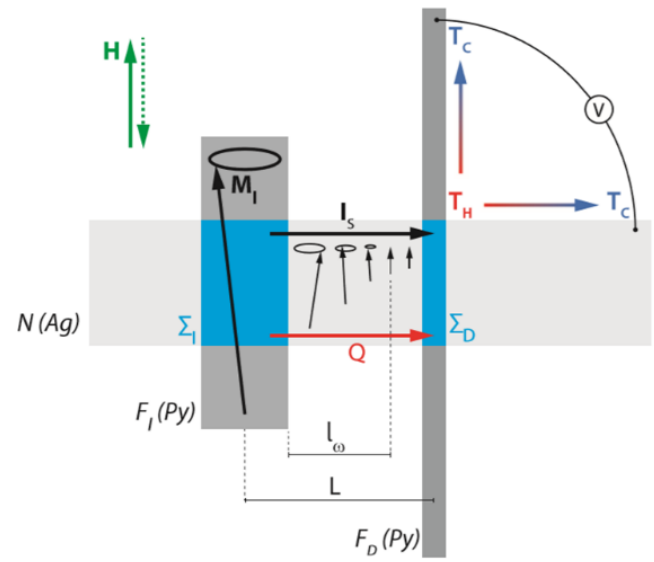

b

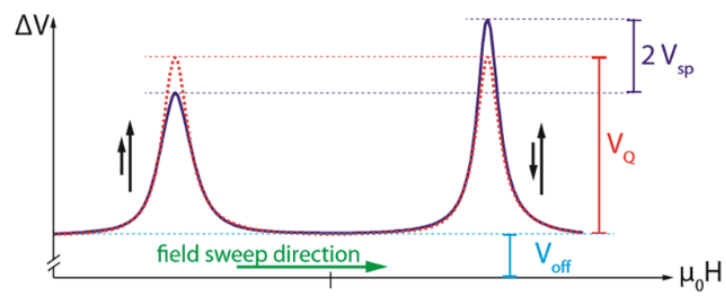

Figure 7.3 Lateral spin valve operated by spin pumping. a, Schematic illustration of lateral spin valve with spin pumping spin injection measurement. $\mathbf{b}$, Non-local spin signal, $V_{\text {sp }}$ for silver based lateral spin valve operated by spin pumping. The voltage due to heating at injector electrode at FMR, $V_{\mathrm{Q}}$ and the offset voltage due to inductive voltage generation in the measurement, $V_{\text {off }}$ contributions to the measured signal are illustrated in the figure. This figure is modified from [178]. 


\section{References}

[1] W. Brütting, Physics of Organic Semiconductors, 2006.

[2] H. Sirringhaus, "Device Physics of Solution-Processed Organic Field-Effect Transistors," Advanced Materials, 2005.

[3] H. Bassler and A. Kohler, "Charge transport in organic semiconductors," Top Curr Chem, 2012.

[4] S. A. Wolf, D. D. Awschalom, R. A. Buhrman, J. M. Daughton, S. Von Molnár, M. L. Roukes, A. Y. Chtchelkanova and D. M. Treger, Spintronics: A spin-based electronics vision for the future, 2001.

[5] G. Binasch, P. Grünberg, F. Saurenbach and W. Zinn, "Enhanced magnetoresistance in layered magnetic structures with antiferromagnetic interlayer exchange," Physical Review B, 1989.

[6] M. N. Baibich, J. M. Broto, A. Fert and F. N. Van Dau, "Giant magnetoresistance of (001) Fe/(001) Cr magnetic superlattices," Physical Review Letters, 1988.

[7] S. S. Parkin, C. Kaiser, A. Panchula, P. M. Rice, B. Hughes, M. Samant and S. H. Yang, "Giant tunnelling magnetoresistance at room temperature with $\mathrm{MgO}$ (100) tunnel barriers," Nature Materials, 2004.

[8] S. Yuasa, T. Nagahama, A. Fukushima, Y. Suzuki and K. Ando, "Giant roomtemperature magnetoresistance in single-crystal Fe/MgO/Fe magnetic tunnel junctions," Nature Materials, 2004.

[9] J. Slonczewski, "Current-driven excitation of magnetic multilayers," Journal of Magnetism and Magnetic Materials, 1996.

[10] "Memory with a spin," Nature Publishing Group, vol. 10, 2015.

[11] J. Sinova, S. O. Valenzuela, J. Wunderlich, C. H. Back and T. Jungwirth, Spin Hall effects, 2015.

[12] J. Wunderlich, B.-G. Park, A. C. Irvine, L. P. Zarbo, E. Rozkotova, P. Nemec, V. Novak, J. Sinova and T. Jungwirth, "Spin Hall Effect Transistor," Science, 2010.

[13] K. Uchida, S. Takahashi, K. Harii, J. Ieda, W. Koshibae, K. Ando, S. Maekawa and E. Saitoh, "Observation of the spin Seebeck effect," Nature, 2008.

[14] I. M. Miron, K. Garello, G. Gaudin, P. J. Zermatten, M. V. Costache, S. Auffret, S. 
Bandiera, B. Rodmacq, A. Schuhl and P. Gambardella, Perpendicular switching of a single ferromagnetic layer induced by in-plane current injection, 2011.

[15] M. Cubukcu, O. Boulle, M. Drouard, K. Garello, C. Onur Avci, I. Mihai Miron, J. Langer, B. Ocker, P. Gambardella and G. Gaudin, "Spin-orbit torque magnetization switching of a three-terminal perpendicular magnetic tunnel junction," Applied Physics Letters, 2014.

[16] L. Liu, T. Moriyama, D. C. Ralph and R. A. Buhrman, "Spin-torque ferromagnetic resonance induced by the spin Hall effect," Phys Rev Lett, 2011.

[17] K. v. Bergmann, "Magnetic bubbles with a twist," Science, 2015.

[18] J. C. Sánchez, L. Vila, G. Desfonds, S. Gambarelli, J. P. Attané, J. M. De Teresa, C. Magén and A. Fert, "Spin-to-charge conversion using Rashba coupling at the interface between non-magnetic materials," Nature Communications, 2013.

[19] S. Meyer, Y. T. Chen, S. Wimmer, M. Althammer, T. Wimmer, R. Schlitz, S. Geprags, H. Huebl, D. Kodderitzsch, H. Ebert, G. E. Bauer, R. Gross and S. T. Goennenwein, "Observation of the spin Nernst effect," Nature Materials, 2017.

[20] M. Cinchetti, V. A. Dediu and L. E. Hueso, Activating the molecular spinterface, 2017.

[21] M. Warner, S. Din, I. S. Tupitsyn, G. W. Morley, A. M. Stoneham, J. A. Gardener, Z. Wu, A. J. Fisher, S. Heutz, C. W. Kay and G. Aeppli, "Potential for spin-based information processing in a thin-film molecular semiconductor," Nature, 2013.

[22] V. A. Dediu, L. E. Hueso, I. Bergenti and C. Taliani, "Spin routes in organic semiconductors," Nature Materials, 2009.

[23] G. Szulczewski, S. Sanvito and M. Coey, "A spin of their own," Nat. Mater., 2009.

[24] H. Sirringhaus, 25th anniversary article: Organic field-effect transistors: The path beyond amorphous silicon, 2014.

[25] F. Al Ma'Mari, T. Moorsom, G. Teobaldi, W. Deacon, T. Prokscha, H. Luetkens, S. Lee, G. E. Sterbinsky, D. A. Arena, D. A. Maclaren, M. Flokstra, M. Ali, M. C. Wheeler, G. Burnell, B. J. Hickey and O. Cespedes, "Beating the Stoner criterion using molecular interfaces," Nature, 2015.

[26] M. Gruber, F. Ibrahim, S. Boukari, H. Isshiki, L. Joly, M. Peter, M. Studniarek, V. Da Costa, H. Jabbar, V. Davesne, U. Halisdemir, J. Chen, J. Arabski, E. Otero, F. Choueikani, K. Chen, P. Ohresser, W. Wulfhekel, F. Scheurer, W. Weber, M. Alouani, E. 
Beaurepaire and M. Bowen, "Exchange bias and room-temperature magnetic order in molecular layers," Nature Materials, 2015.

[27] T. L. Francis, O.”. Mermer, G. Veeraraghavan and M. Wohlgenannt, "Large magnetoresistance at room temperature in semiconducting polymer sandwich devices," New Journal of Physics, 2004.

[28] Ö. Mermer, G. Veeraraghavan, T. L. Francis, Y. Sheng, D. T. Nguyen, M. Wohlgenannt, A. Köhler, M. K. Al-Suti and M. S. Khan, "Large magnetoresistance in nonmagnetic $\pi$ conjugated semiconductor thin film devices," Physical Review B - Condensed Matter and Materials Physics, 2005.

[29] P. A. Bobbert, T. D. Nguyen, F. W. Van Oost, B. Koopmans and M. Wohlgenannt, "Bipolaron mechanism for organic magnetoresistance," Physical Review Letters, 2007.

[30] T. D. Nguyen, G. Hukic-Markosian, F. Wang, L. Wojcik, X. G. Li, E. Ehrenfreund and Z. V. Vardeny, "Isotope effect in spin response of $\pi$-conjugated polymer films and devices," Nature Materials, 2010.

[31] G. Veeraraghavan, T. D. Nguyen, Y. Sheng, Ö. Mermer and M. Wohlgenannt, "An $8 \times$ 8 pixel array pen-input OLED screen based on organic magnetoresistance," IEEE Transactions on Electron Devices, 2007.

[32] Z. H. Xiong, D. Wu, Z. V. Vardeny and J. Shi, "Giant magnetoresistance in organic spinvalves," Nature, 2004.

[33] X. Zhang, S. Mizukami, T. Kubota, Q. Ma, M. Oogane, H. Naganuma, Y. Ando and T. Miyazaki, "Observation of a large spin-dependent transport length in organic Spin valves at room temperature," Nature Communications, 2013.

[34] S. Mooser, J. Cooper, K. Banger, J. Wunderlich and H. Sirringhaus, "Spin injection and transport in a solution-processed organic semiconductor at room temperature," Physical Review B - Condensed Matter and Materials Physics, 2012.

[35] S. Majumdar and H. S. Majumdar, "Decay in spin diffusion length with temperature in organic semiconductors - An insight of possible mechanisms," Synthetic Metals, 2013.

[36] F. Li, T. Li, F. Chen and F. Zhang, "Excellent spin transport in spin valves based on the conjugated polymer with high carrier mobility," Scientific Reports, 2015.

[37] S. Majumdar and H. S. Majumdar, "On the origin of decay of spin current with temperature in organic spintronic devices," Organic Electronics: physics, materials, 
applications, 2012.

[38] N. A. Morley, A. Rao, D. Dhandapani, M. R. Gibbs, M. Grell and T. Richardson, "Room temperature organic spintronics," in Journal of Applied Physics, 2008.

[39] M. Grünewald, M. Wahler, F. Schumann, M. Michelfeit, C. Gould, R. Schmidt, F. Würthner, G. Schmidt and L. W. Molenkamp, "Tunneling anisotropic magnetoresistance in organic spin valves," Physical Review B - Condensed Matter and Materials Physics, 2011.

[40] M. Grünewald, R. Göckeritz, N. Homonnay, F. Würthner, L. W. Molenkamp and G. Schmidt, "Vertical organic spin valves in perpendicular magnetic fields," Physical Review B - Condensed Matter and Materials Physics, 2013.

[41] M. Galbiati, S. Tatay, S. Delprat, H. L. Khanh, B. Servet, C. Deranlot, S. Collin, P. Seneor, R. Mattana and F. Petroff, "Is spin transport through molecules really occurring in organic spin valves? A combined magnetoresistance and inelastic electron tunnelling spectroscopy study," Applied Physics Letters, 2015.

[42] J. H. Shim, K. V. Raman, Y. J. Park, T. S. Santos, G. X. Miao, B. Satpati and J. S. Moodera, "Large spin diffusion length in an amorphous organic semiconductor," Physical Review Letters, 2008.

[43] K. S. Li, Y. M. Chang, S. Agilan, J. Y. Hong, J. C. Tai, W. C. Chiang, K. Fukutani, P. A. Dowben and M. T. Lin, "Organic spin valves with inelastic tunneling characteristics," Physical Review B - Condensed Matter and Materials Physics, 2011.

[44] X. Sun, A. Bedoya-Pinto, Z. Mao, M. Gobbi, W. Yan, Y. Guo, A. Atxabal, R. Llopis, G. Yu, Y. Liu, A. Chuvilin, F. Casanova and L. E. Hueso, "Active Morphology Control for Concomitant Long Distance Spin Transport and Photoresponse in a Single Organic Device," Advanced Materials, 2016.

[45] M. Cinchetti, K. Heimer, J. P. Wüstenberg, O. Andreyev, M. Bauer, S. Lach, C. Ziegler, Y. Gao and M. Aeschlimann, "Determination of spin injection and transport in a ferromagnet/organic semiconductor heterojunction by two-photon photoemission," Nature Materials, 2009.

[46] T. D. Nguyen, E. Ehrenfreund and Z. V. Vardeny, "Spin-polarized light-emitting diode based on an organic bipolar spin valve.," Science (New York, N.Y.), 2012.

[47] A. J. Drew, J. Hoppler, L. Schulz, F. L. Pratt, P. Desai, P. Shakya, T. Kreouzis, W. P. Gillin, A. Suter, N. A. Morley, V. K. Malik, A. Dubroka, K. W. Kim, H. Bouyanfif, F. 
Bourqui, C. Bernhard, R. Scheuermann, G. J. Nieuwenhuys, T. Prokscha and E. Morenzoni, "Direct measurement of the electronic spin diffusion length in a fully functional organic spin valve by low-energy muon spin rotation," Nature Materials, 2009.

[48] X. Lou, C. Adelmann, S. A. Crooker, E. S. Garlid, J. Zhang, K. S. M. Reddy, S. D. Flexner, C. J. Palmstrøm and P. A. Crowell, "Electrical detection of spin transport in lateral ferromagnet-semiconductor devices," Nature Physics, 2007.

[49] F. J. Jedema, H. B. Heersche, A. T. Filip, J. J. A. Baselmans and B. J. van Wees, "Electrical detection of spin precession in a metallic mesoscopic spin valve," Nature, vol. 416, p. 713-716, 2002.

[50] A. Avsar, T.-Y. Yang, S. Bae, J. Balakrishnan, F. Volmer, M. Jaiswal, Z. Yi, S. R. Ali, G. Güntherodt, B. H. Hong, B. Beschoten and B. Özyilmas, "Toward Wafer Scale Fabrication of Graphene Based Spin Valve Devices," Nano Lett., 2011.

[51] Y. Zhou, W. Han, L.-T. Chang, F. Xiu, M. Wang, M. Oehme, I. Fischer, J. Schulze, R. Kawakami and K. Wang, "Electrical spin injection and transport in germanium," Physical Review B, vol. 84, p. 125323, 2011.

[52] G. Salis, A. Fuhrer, R. R. Schlittler, L. Gross and S. F. Alvarado, "Temperature dependence of the nonlocal voltage in an Fe/GaAs electrical spin-injection device," Physical Review B - Condensed Matter and Materials Physics, 2010.

[53] K. Fujiwara, Y. Fukuma, J. Matsuno, H. Idzuchi, Y. Niimi, Y. Otani and H. Takagi, "5diridium oxide as a material for spin-current detection," Nature Communications, 2013.

[54] S. Martin, A. Dodabalapur, Z. Bao, B. Crone, H. E. Katz, W. Li, A. Passner and J. A. Rogers, "Flicker noise properties of organic thin-film transistors," Journal of Applied Physics, 2000.

[55] H.-J. Jang and C. A. Richter, "Organic Spin-Valves and Beyond: Spin Injection and Transport in Organic Semiconductors and the Effect of Interfacial Engineering," Advanced Materials, 2017.

[56] E. Saitoh, M. Ueda, H. Miyajima and G. Tatara, "Conversion of spin current into charge current at room temperature: Inverse spin-Hall effect," Applied Physics Letters, vol. 88, p. 182509, 2006.

[57] K. Ando and E. Saitoh, "Inverse spin-Hall effect in palladium at room temperature," 
Journal of Applied Physics, 2010.

[58] S. Dushenko, H. Ago, K. Kawahara, T. Tsuda, S. Kuwabata, T. Takenobu, T. Shinjo, Y. Ando and M. Shiraishi, "Gate-Tunable Spin-Charge Conversion and the Role of SpinOrbit Interaction in Graphene," Physical Review Letters, 2016.

[59] K. Ando and E. Saitoh, "Observation of the inverse spin Hall effect in silicon," Nature commun., 2012.

[60] K. Ando, S. Takahashi, J. Ieda, H. Kurebayashi, T. Trypiniotis, C. H. Barnes, S. Maekawa and E. Saitoh, "Electrically tunable spin injector free from the impedance mismatch problem," Nature Materials, 2011.

[61] K. Ando, S. Watanabe, S. Mooser, E. Saitoh and H. Sirringhaus, "Solution-processed organic spin-charge converter," Nature Materials, 2013.

[62] S. Watanabe, K. Ando, K. Kang, S. Mooser, Y. Vaynzof, H. Kurebayashi, E. Saitoh and H. Sirringhaus, "Polaron spin current transport in organic semiconductors," Nature Physics, 2014.

[63] G. A. Prinz, "Magnetoelectronics," Science (New York, N.Y.), 1998.

[64] G. Schmidt, D. Ferrand, L. Molenkamp, A. Filip and B. van Wees, "Fundamental obstacle for electrical spin injection from a ferromagnetic metal into a diffusive semiconductor," Physical Review B - Condensed Matter and Materials Physics, 2000.

[65] A. Fert and H. Jaffrès, "Conditions for efficient spin injection from a ferromagnetic metal into a semiconductor," Physical Review B - Condensed Matter and Materials Physics, 2001.

[66] J. Bass and W. P. Pratt, "Current-perpendicular (CPP) magnetoresistance in magnetic metallic multilayers," Journal of Magnetism and Magnetic Materials, 1999.

[67] A. Fert and L. Piraux, "Magnetic nanowires," J. Magn. Magn. Mat., 1999.

[68] F. J. Jedema, A. T. Filip and B. J. Van Wees, Electrical spin injection and accumulation at room temperature in an all-metal mesoscopic spin valve, 2001.

[69] D. J. Monsma and S. S. Parkin, "Temporal evolution of spin-polarization in ferromagnetic tunnel junctions," Applied Physics Letters, 2000.

[70] T. Valet and A. Fert, "Theory of the perpendicular magnetoresistance in magnetic multilayers," Physical Review B, 1993.

[71] X. Lou, C. Adelmann, M. Furis, S. A. Crooker, C. J. Palmstr??m and P. A. Crowell, 
"Electrical detection of Spin accumulation at a ferromagnet-semiconductor interface," Physical Review Letters, 2006.

[72] G. Schmidt, "Tunneling and Resistive Switching in Organic Spin Valves," in World Scientific Reference on Spin in Organics, 2018, pp. 225-280 .

[73] C. Barraud, P. Seneor, R. Mattana, S. Fusil, K. Bouzehouane, C. Deranlot, P. Graziosi, L. Hueso, I. Bergenti, V. Dediu, F. Petroff and A. Fert, "Unravelling the role of the interface for spin injection into organic semiconductors," Nature Physics, 2010.

[74] R. Lin, F. Wang, J. Rybicki, M. Wohlgenannt and K. A. Hutchinson, "Distinguishing between tunneling and injection regimes of ferromagnet/organic semiconductor/ferromagnet junctions," Physical Review B - Condensed Matter and Materials Physics, 2010.

[75] J. W. Yoo, H. W. Jang, V. N. Prigodin, C. Kao, C. B. Eom and A. J. Epstein, "Tunneling vs. giant magnetoresistance in organic spin valve," Synthetic Metals, 2010.

[76] T. Kamiya, Y. Kawasugi, M. Ara and H. Tada, "Nonlocal magnetoresistance measurements of the organic zero-gap conductor $\alpha$-(BEDT-TTF)2 I3," Physical Review B, 2017.

[77] W. Yan, O. Txoperena, R. Llopis, H. Dery, L. E. Hueso and F. Casanova, "A twodimensional spin field-effect switch," Nature Communications, 2016.

[78] P. Chuang, S. C. Ho, L. W. Smith, F. Sfigakis, M. Pepper, C. H. Chen, J. C. Fan, J. P. Griffiths, I. Farrer, H. E. Beere, G. A. Jones, D. A. Ritchie and T. M. Chen, "All-electric all-semiconductor spin field-effect transistors," Nature Nanotechnology, 2015.

[79] B. Wang, "Moving forward, spin goes sideways," 2011. [Online]. Available: https://www.nextbigfuture.com/2011/10/notre-dame-researchers-make.html.

[80] T. Kimura and Y. Otani, "Spin transport in lateral ferromagnetic/nonmagnetic hybrid structures," J. Phys.: Condens. Matter, vol. 19, p. 165216, 2007.

[81] W. Han, K. M. McCreary, K. Pi, W. H. Wang, Y. Li, H. Wen, J. R. Chen and R. K. Kawakami, Spin transport and relaxation in graphene, 2012.

[82] W. Han, R. K. Kawakami, M. Gmitra and J. Fabian, Graphene spintronics, 2014.

[83] S. P. Dash, S. Sharma, R. S. Patel, M. P. De Jong and R. Jansen, "Electrical creation of spin polarization in silicon at room temperature," Nature, 2009.

[84] Y. Tserkovnyak, A. Brataas, G. E. Bauer and B. I. Halperin, "Nonlocal magnetization 
dynamics in ferromagnetic heterostructures," Reviews of Modern Physics, 2005.

[85] K. Ando, S. Takahashi, J. Ieda, Y. Kajiwara, H. Nakayama, T. Yoshino, K. Harii, Y. Fujikawa, M. Matsuo, S. Maekawa and E. Saitoh, "Inverse spin-Hall effect induced by spin pumping in metallic system," in Journal of Applied Physics, 2011.

[86] C. Kittel, "Ferromagnetic resonance," Physica, 1951.

[87] Y. Tserkovnyak, A. Brataas and G. E. Bauer, "Spin pumping and magnetization dynamics in metallic multilayers," Physical Review B - Condensed Matter and Materials Physics, 2002.

[88] Y. Tserkovnyak, A. Brataas and G. E. Bauer, "Enhanced Gilbert Damping in Thin Ferromagnetic Films," Physical Review Letters, 2002.

[89] J. Bjorken and S. Drell, Relativistic Quantum Mechanics, 1964: McGraw-Hill inc.

[90] Y. K. Kato, R. C. Myers, A. C. Gossard and D. D. Awschalom, "Observation of the spin hall effect in semiconductors," Science, 2004.

[91] N. Nagaosa, J. Sinova, S. Onoda, A. H. MacDonald and N. P. Ong, "Anomalous Hall effect," Reviews of Modern, 2009.

[92] S. O. Valenzuela and M. Tinkham, "Direct electronic measurement of the spin Hall effect," Nature, 2006.

[93] S. O. Valenzuela and M. Tinkham, "Electrical detection of spin currents: The spincurrent induced Hall effect (invited)," in Journal of Applied Physics, 2007.

[94] X. Wang, C. O. Pauyac and A. Manchon, "Spin-orbit-coupled transport and spin torque in a ferromagnetic heterostructure," Physical Review B - Condensed Matter and Materials Physics, 2014.

[95] M. Obstbaum, M. Härtinger, H. G. Bauer, T. Meier, F. Swientek, C. H. Back and G. Woltersdorf, "Inverse spin Hall effect in Ni81Fe19/normal-metal bilayers," Physical Review B - Condensed Matter and Materials Physics, 2014.

[96] E. Shikoh, K. Ando, K. Kubo, E. Saitoh, T. Shinjo and M. Shiraishi, "Spin-pumpinduced spin transport in p-type Si at room temperature," Physical Review Letters, 2013.

[97] R. Ohshima, Y. Ando, K. Matsuzaki, T. Susaki, M. Weiler, S. Klingler, H. Huebl, E. Shikoh, T. Shinjo, S. T. B. Goennenwein and M. Shiraishi, "Strong evidence for delectron spin transport at room temperature at a $\mathrm{LaAlO} 3 / \mathrm{SrTiO} 3$ interface," Nature Materials, 2017. 
[98] S. Dushenko, M. Koike, Y. Ando, T. Shinjo, M. Myronov and M. Shiraishi, "Experimental Demonstration of Room-Temperature Spin Transport in n-Type Germanium Epilayers," Physical Review Letters, 2015.

[99] Z. Tang, E. Shikoh, H. Ago, K. Kawahara, Y. Ando, T. Shinjo and M. Shiraishi, "Dynamically generated pure spin current in single-layer graphene," Physical Review B - Condensed Matter and Materials Physics, 2013.

[100] A. Yamamoto, Y. Ando, T. Shinjo, T. Uemura and M. Shiraishi, "Spin transport and spin conversion in compound semiconductor with non-negligible spin-orbit interaction," Physical Review B - Condensed Matter and Materials Physics, 2015.

[101] T. Yamamoto, T. Seki, S. Ono and K. Takanashi, "Characterization of spin pumping effect in Permalloy/Cu/Pt microfabricated lateral devices," in Journal of Applied Physics, 2014.

[102] F. Bloch, "Nuclear induction," Physical Review, 1946.

[103] H. C. Torrey, "Bloch equations with diffusion terms," Physical Review, 1956.

[104] I. Žutić, J. Fabian and S. D. Sarma, Spintronics: Fundamentals and applications, 2004.

[105] E. L. Hahn, "Spin echoes," Physical Review, 1950.

[106] R. J. Elliott, "Theory of the effect of spin-Orbit coupling on magnetic resonance in some semiconductors," Physical Review, 1954.

[107] Y. Yafet, in Solid State Physics, New York, Academic, 1963.

[108] J. L. Cheng, M. W. Wu and J. Fabian, "Theory of the spin relaxation of conduction electrons in silicon," Physical Review Letters, 2010.

[109] D. J. Lépine, "Spin resonance of localized and delocalized electrons in phosphorusdoped silicon between 20 and 30 K," Physical Review B, 1970.

[110] I. Appelbaum, B. Huang and D. J. Monsma, "Electronic measurement and control of spin transport in silicon," Nature, 2007.

[111] G. Dresselhaus, "Spin-Orbit Coupling Effects in Zinc Blende Structures," Phys.Rev., 1955.

[112] Y. A. Bychkov and E. I. Rashba, "Oscillatory effects and the magnetic susceptibility of carriers in inversion layers," Journal of Physics C: Solid State Physics, 1984.

[113] A. Manchon, H. C. Koo, J. Nitta, S. M. Frolov and R. A. Duine, "New perspectives for Rashba spin-orbit coupling," Nature Materials, 2015. 
[114] H. Zhao, M. Mower and G. Vignale, "Ambipolar spin diffusion and D'yakonov-Perel' spin relaxation in GaAs quantum wells," Physical Review B - Condensed Matter and Materials Physics, 2009.

[115] J. H. Buß, J. Rudolph, S. Starosielec, A. Schaefer, F. Semond, Y. Cordier, A. D. Wieck and D. Hägele, "Dyakonov-Perel electron spin relaxation in a wurtzite semiconductor: From the nondegenerate to the highly degenerate regime," Physical Review B Condensed Matter and Materials Physics, 2011.

[116] M. A. Brand, A. Malinowski, O. Z. Karimov, P. A. Marsden, R. T. Harley, A. J. Shields, D. Sanvitto, D. A. Ritchie and M. Y. Simmons, "Precession and Motional Slowing of Spin Evolution in a High Mobility Two-Dimensional Electron Gas," Physical Review Letters, 2002.

[117] M. I. Dyakonov and V. I. Perel, "Optical orientation in a system of electrons and lattice nuclei in semiconductors," Soviet Journal of Experimental and Theoretical Physics, vol. 38, pp. 177-183, 1974 .

[118] C. P. Slichter, Principles of Magnetic Resonance, Berlin: Springer, 1989.

[119] H. M. McConnell, "Indirect hyperfine interactions in the paramagnetic resonance spectra of aromatic free radicals," The Journal of Chemical Physics, 1956.

[120] J. Kalinowski, M. Cocchi, D. Virgili, P. Di Marco and V. Fattori, "Magnetic field effects on emission and current in Alq3-based electroluminescent diodes," Chemical Physics Letters, 2003.

[121] E. L. Frankevich, A. A. Lymarev, I. Sokolik, F. E. Karasz, S. Blumstengel, R. H. Baughman and H. H. Hörhold, "Polaron-pair generation in poly(phenylene vinylenes)," Physical Review B, 1992.

[122] Ö. Mermer, G. Veeraraghavan, T. L. Francis and M. Wohlgenannt, "Large magnetoresistance at room-temperature in small-molecular-weight organic semiconductor sandwich devices," Solid State Communications, 2005.

[123] Y. Sheng, T. D. Nguyen, G. Veeraraghavan, Ö. Mermer, M. Wohlgenannt, S. Qiu and U. Scherf, "Hyperfine interaction and magnetoresistance in organic semiconductors," Physical Review B - Condensed Matter and Materials Physics, 2006.

[124] N. J. Rolfe, M. Heeney, P. B. Wyatt, A. J. Drew, T. Kreouzis and W. P. Gillin, "Elucidating the role of hyperfine interactions on organic magnetoresistance using deuterated aluminium tris(8-hydroxyquinoline)," Physical Review B - Condensed 
Matter and Materials Physics, 2009.

[125] Z. G. Yu, "Suppression of the hanle effect in organic spintronic devices," Physical Review Letters, 2013.

[126] Z. G. Yu, F. Ding and H. Wang, "Hyperfine interaction and its effects on spin dynamics in organic solids," PHYSICAL REVIEW B, 2013.

[127] Z. G. Yu, "Spin-orbit coupling, spin relaxation, and spin diffusion in organic solids," Physical Review Letters, 2011.

[128] Z. Yu, "Spin-orbit coupling and its effects in organic solids," Physical Review B, 2012.

[129] Z. G. Yu, "Spin transport and the Hanle effect in organic spintronics," Nanoelectronics and Spintronics, 2015.

[130] H. Mu, D. Klotzkin, A. De Silva, H. P. Wagner, D. White and B. Sharpton, "Temperature dependence of electron mobility, electroluminescence and photoluminescence of Alq3 in OLED," Journal of Physics D: Applied Physics, 2008.

[131] R. Göckeritz, N. Homonnay, A. Müller, T. Richter, B. Fuhrmann and G. Schmidt, "Nanosized perpendicular organic spin-valves," Applied Physics Letters, 2015.

[132] Z. G. Yu, "Impurity-band transport in organic spin valves," Nature Communications, 2014.

[133] D. Venkateshvaran, M. Nikolka, A. Sadhanala, V. Lemaur, M. Zelazny, M. Kepa, M. Hurhangee, K. A. J., V. Pecunia, I. Nasrallah, R. I. K. Broch, I. McCulloch, D. Emin, Y. Olivier, J. Cornil, D. Beljonne and H. Sirringhaus, "Approaching disorder-free transport in high-mobility conjugated polymers," Nature, vol. 515, p. 384-388, 2014.

[134] E. I. Rashba, "Theory of electrical spin injection: Tunnel contacts as a solution of the conductivity mismatch problem," Physical Review B - Condensed Matter and Materials Physics, 2000.

[135] M. Drögeler, C. Franzen, F. Volmer, T. Pohlmann, L. Banszerus, M. Wolter, K. Watanabe, T. Taniguchi, C. Stampfer and B. Beschoten, "Spin Lifetimes Exceeding 12 ns in Graphene Nonlocal Spin Valve Devices," Nano Letters, 2016.

[136] T. L. A. Tran, T. Q. Le, J. G. Sanderink, W. G. Van Der Wiel and M. P. De Jong, "The multistep tunneling analogue of conductivity mismatch in organic spin valves," Advanced Functional Materials, 2012.

[137] T. Kimura, Y. Otani and J. Hamrle, "Enhancement of spin accumulation in a 
nonmagnetic layer by reducing junction size," Physical Review B - Condensed Matter and Materials Physics, 2006.

[138] T. V. A. G. De Oliveira, M. Gobbi, J. M. Porro, L. E. Hueso and A. M. Bittner, "Charge and spin transport in PEDOT:PSS nanoscale lateral devices," Nanotechnology, 2013.

[139] H.-J. Jang and C. A. Richter, "Organic Spin-Valves and Beyond: Spin Injection and Transport in Organic Semiconductors and the Effect of Interfacial Engineering," Advanced Materials, vol. 29, p. 1602739, 2017.

[140] W. Wang, A. Narayan, L. Tang, K. Dolui, Y. Liu, X. Yuan, Y. Jin, Y. Wu, I. Rungger, S. Sanvito and F. Xiu, "Spin-Valve Effect in NiFe/MoS2/NiFe Junctions," Nano Letters, 2015.

[141] S. Ding, Y. Tian, Y. Li, W. Mi, H. Dong, X. Zhang, W. Hu and D. Zhu, "Inverse Magnetoresistance in Polymer Spin Valves," ACS Applied Materials and Interfaces, 2017.

[142] J. W. Yoo, H. W. Jang, V. N. Prigodin, C. Kao, C. B. Eom and A. J. Epstein, "Giant magnetoresistance in ferromagnet/organic semiconductor/ferromagnet heterojunctions," Physical Review B - Condensed Matter and Materials Physics, 2009.

[143] W. Xu, G. J. Szulczewski, P. LeClair, I. Navarrete, R. Schad, G. Miao, H. Guo and A. Gupta, "Tunneling magnetoresistance observed in La0.67Sr0.33MnO3/organic molecule/Co junctions," Applied Physics Letters, 2007.

[144] B. Lee, Y. Chen, D. Fu, H. T. Yi, K. Czelen, H. Najafov and V. Podzorov, "Trap healing and ultralow-noise Hall effect at the surface of organic semiconductors," Nature Materials, 2013.

[145] S. W. Jiang, S. Liu, P. Wang, Z. Z. Luan, X. D. Tao, H. F. Ding and D. Wu, "ExchangeDominated Pure Spin Current Transport in Alq3 Molecules," Physical Review Letters, 2015.

[146] J. B. Mendes, O. Alves Santos, J. P. Gomes, H. S. Assis, J. F. Felix, R. L. RodríguezSuárez, S. M. Rezende and A. Azevedo, "Efficient spin transport through polyaniline," Physical Review B, 2017.

[147] M. Kimata, D. Nozaki, Y. Niimi, H. Tajima and Y. Otani, "Spin relaxation mechanism in a highly doped organic polymer film," Physical Review B, 2015.

[148] Y. Tani, Y. Teki and E. Shikoh, "Spin-pump-induced spin transport in a thermally 
evaporated pentacene film," Applied Physics Letters, 2015.

[149] D. Sun, K. J. Van Schooten, M. Kavand, H. Malissa, C. Zhang, M. Groesbeck, C. Boehme and Z. Valy Vardeny, "Inverse spin Hall effect from pulsed spin current in organic semiconductors with tunable spin-orbit coupling," Nature Materials, 2016.

[150] M. A. Lampert and P. Mark, Current Injection in Solids, New York: Academic, 1970.

[151] P. W. M. Blom, M. J. M. De Jong and J. J. M. Vleggaar, "Electron and hole transport in poly(p-phenylene vinylene) devices," Applied Physics Letters, 1996.

[152] M. Gruber, S.-H. Jung, S. Schott, D. Venkateshvaran, A. J. Kronemeijer, J. W. Andreasen, C. R. McNeill, W. W. H. Wong, M. Shahid, M. Heeney, J.-K. Lee and H. Sirringhaus, "Enabling high-mobility, ambipolar charge-transport in a DPPbenzotriazole copolymer by side-chain engineering," Chem. Sci., 2015.

[153] R. Steyrleuthner, M. Schubert, F. Jaiser, J. C. Blakesley, Z. Chen, A. Facchetti and D. Neher, "Bulk electron transport and charge injection in a high mobility n-type semiconducting polymer," Advanced Materials, 2010.

[154] C. Liu, Y. Xu and Y. Y. Noh, Contact engineering in organic field-effect transistors, 2015.

[155] C. Wang, L. H. Jimison, L. Goris, I. McCulloch, M. Heeney, A. Ziegler and A. Salleo, "Microstructural origin of high mobility in high-performance poly(thieno-thiophene) thin-film transistors," Advanced Materials, 2010.

[156] T. Liu and A. Troisi, "Understanding the microscopic origin of the very high charge mobility in PBTTT: Tolerance of thermal disorder," Advanced Functional Materials, 2014.

[157] H. Nakayama, T. Yamamoto, H. An, K. Tsuda, Y. Einaga and K. Ando, "Molecular engineering of Rashba spin-charge converter," Science Advances, vol. 4, p. eaar3899, 2018.

[158] Y. Tserkovnyak, A. Brataas and G. E. W. Bauer, "Enhanced Gilbert Damping in Thin Ferromagnetic Films," Physical Review Letters, vol. 88, p. 117601, 2002.

[159] R. H. Silsbee, A. Janossy and P. Monod, "Coupling between ferromagnetic and conduction-spin-resonance modes at a ferromagnetic-normal-metal interface," Physical Review B, vol. 19, p. 4382, 1979.

[160] S. Maekawa, S. O. Valenzuela, E. Saitoh and T. Kimura, Spin Current, Oxford: Oxford 
University Press, 2012, pp. 87-135.

[161] A. Brataas, Y. Tserkovnyak, G. E. W. Bauer and B. I. Halperin, "Spin battery operated by ferromagnetic resonance," Physical Review B - Condensed Matter and Materials Physics, 2002.

[162] K. Kang, S. Watanabe, K. Broch, A. Sepe, A. Brown, I. Nasrallah, M. Nikolka, Z. Fei, M. Heeney, D. Matsumoto, K. Marumoto, H. Tanaka, S. Kuroda and H. Sirringhaus, "2D coherent charge transport in highly ordered conducting polymers doped by solid state diffusion," Nature Materials, vol. 15, p. 896, 2016.

[163] D. Alberga, P. Aurelie, I. Ciofini, G. F. Mangiatordi, G. Lattanzi and C. Adamo, "Morphological and charge transport properties of amorphous and crystalline $\mathrm{P} 3 \mathrm{HT}$ and PBTTT: insights from theory," Physical Chemistry Chemical Physics, vol. 17, p. 18742, 2015.

[164] T. Morita, "Spin diffusion in the heisenberg magnets at infinite temperature," Physical Review B, 1972.

[165] M. Boeckmann, T. Schemme, D. H. de Jong, C. Denz, A. Heuerc and N. L. Doltsinis, "Structure of P3HT crystals, thin films, and solutions by UV/Vis spectral analysis," Physical Chemistry Chemical Physics, vol. 17, p. 28616, 2015.

[166] W. Luzny, "X-ray diffraction and computer modelling study of the structure and conformation of poly(3-decylthiophene)," Acta Crystallogr. Sect. B Struct. Sci., vol. 51, no. 2, p. 255-260, 1995.

[167] A. R. O'Dea, A. F. Curtis, N. J. B. Green, C. R. Timmel and P. J. Hore, "Influence of Dipolar Interactions on Radical Pair Recombination Reactions Subject to Weak Magnetic Fields," Journal of Physical Chemistry A, vol. 109, no. 5, p. 869-873, 2005.

[168] A. Riminucci, M. Prezioso, C. Pernechele, P. Graziosi, I. Bergenti, R. Cecchini, M. Calbucci, M. Solzi and V. A. Dediu, "Hanle effect missing in a prototypical organic spintronic device," Applied Physics Letters, vol. 102, p. 092407, 2013.

[169] H. Sakimura, T. Matsumoto and K. Ando, "Spin rectification induced by dynamical Hanle effect," Applied Physics Letters, vol. 103, p. 132402, 2013.

[170] H. Minemawari, T. Yamada, H. Matsui, J. Y. Tsutsumi, S. Haas, R. Chiba, R. Kumai and T. Hasegawa, "Inkjet printing of single-crystal films," Nature, 2011.

[171] J. Tsurumi, H. Matsui, T. Kubo, R. Häusermann, C. Mitsui, T. Okamoto, S. Watanabe 
and J. Takeya, "Coexistence of ultra-long spin relaxation time and coherent charge transport in organic single-crystal semiconductors," Nature Physics, 2017.

[172] T. Hasegawa and J. Takeya, "Organic field-effect transistors using single crystals," Science and Technology of Advanced Materials, 2009.

[173] H. N. Tsao, D. M. Cho, I. Park, M. R. Hansen, A. Mavrinskiy, D. Y. Yoon, R. Graf, W. Pisula, H. W. Spiess and K. Müllen, "Ultrahigh mobility in polymer field-effect transistors by design," Journal of the American Chemical Society, 2011.

[174] Y. Yamashita, F. Hinkel, T. Marszalek, W. Zajaczkowski, W. Pisula, M. Baumgarten, H. Matsui, K. M??llen and J. Takeya, "Mobility Exceeding $10 \mathrm{~cm} 2 /(\mathrm{V} ? ? \mathrm{~s})$ in DonorAcceptor Polymer Transistors with Band-like Charge Transport," Chemistry of Materials, 2016.

[175] A. Dankert and S. P. Dash, "Electrical gate control of spin current in van der Waals heterostructures at room temperature," Nature Communications, 2017.

[176] H. C. Koo, J. H. Kwon, J. Eom, J. Chang, S. H. Han and M. Johnson, "Control of spin precession in a spin-injected field effect transistor.," Science (New York, N.Y.), 2009.

[177] S. Datta and B. Das, "Electronic analog of the electro-optic modulator," Applied Physics Letters, 1990.

[178] N. Kuhlmann, C. Swoboda, A. Vogel, T. Matsuyama and G. Meier, "All-metal lateral spin valve operated by spin pumping," Physical Review B - Condensed Matter and Materials Physics, 2013.

[179] H. Sirringhaus, N. Tessler and R. H. Friend, "Integrated Optoelectronic Devices Based on Conjugated Polymers," Science, vol. 280, no. 5370, pp. 1741-1744, 1998.

[180] P. Nichols, N. Govind, E. J. Bylaska and W. A. de Jong, "Gaussian Basis Set and Planewave Relativistic Spin-Orbit Methods in NWChem," J. Chem. Theory Comput., vol. 5, no. 3, p. 491-499, 2009.

[181] M. Valiev, E. J. Bylaska, N. Govind, K. Kowalski, T. P. Straatsma, H. J. Van Dam, D. Wang, J. Nieplocha, E. Apra, T. L. Windus and W. A. De Jong, "NWChem: A comprehensive and scalable open-source solution for large scale molecular simulations," Computer Physics Communications, 2010.

[182] C. Adamo and V. Barone, "Toward chemical accuracy in the computation of NMR shieldings: the PBE0 model," Chemical Physics Letters, vol. 298, no. 1-3, p. 113-119, 
1998.

[183] R. Steyrleuthner, Y. Zhang, L. Zhang, F. Kraffert, B. P. Cherniawski, R. Bittl, A. L. Briseno, J.-L. Bredas and J. Behrends, "Impact of morphology on polaron delocalization in a semicrystalline conjugated polymer," Phys. Chem. Chem. Phys., 2017.

[184] F. Neese, "The ORCA program system," Wiley Interdiscip. Rev. Comput. Mol. Sci., vol. 2, no. 1, p. 73-78, 2012.

[185] W. Wagemans, A. J. Schellekens, M. Kemper, F. L. Bloom, P. A. Bobbert and B. Koopmans, "Spin-spin interactions in organic magnetoresistance probed by angledependent measurement," Physical Review B, vol. 106, p. 196802, 2011.

[186] N. Bloembergen, "On the interaction of nuclear spins in a crystalline lattice," Physica, vol. 15, p. $386,1949$.

[187] J. Cardellino, N. Scozzaro, M. Herman, A. Berger, C. Zhang, K. C. Fong, C. Jayaprakash, D. V. Pelekhov and P. C. Hammel, "The effect of spin transport on spin lifetime in nanoscale systems," Nature Nanotechnology, vol. 9, p. 343, 2014.

[188] I. M. Nolden and R. J. Silbey, "Simulation of spin diffusion in a disordered system," Physical Review B, vol. 54, p. 381, 1996.

[189] R. Johnson and R. Silsbee, "Coupling of electronic charge and spin at a ferromagneticparamagnetic metal interface," Physical Review B, vol. 37, pp. 5312-5325, 1988.

[190] M. Johnson and R. H. Silsbee, "Interfacial charge-spin coupling: Injection and detection of spin magnetization in metals," Physical Review Letters, vol. 55, pp. 1790-1793, 1985.

[191] Y. Fukuma, L. Wang, H. Idzuchi, S. Takahashi, S. Maekawa and Y. Otani, "Giant enhancement of spin accumulation and long-distance spin precession in metallic lateral spin valves," Nature Communications, vol. 10, pp. 527-531, 2011.

[192] Y. Karpov, T. Erdmann, I. Raguzin, M. Al-Hussein, M. Binner, U. Lappan, M. Stamm, K. L. Gerasimov, T. Beryozkina, V. Bakulev, D. V. Anokhin, D. A. Ivanov, F. Günther, S. Gemming, G. Seifert, B. Voit, R. Di Pietro and A. Kiriy, "High Conductivity in Molecularly p-Doped Diketopyrrolopyrrole-Based Polymer: The Impact of a High Dopant Strength and Good Structural Order," Advanced Materials, vol. 28, pp. 6003$10,2016$. 
Appendices 


\section{Appendix A}

\section{Theoretical modelling details}

\section{Modelling}

In order to calculate the Spin diffusion length (SDL) for PBTTT, we adopt the model in Ref [125] to a morphology with quasi one-dimensional transport properties. The SD is calculated from

$$
\lambda_{S}=\sqrt{D T_{1}}
$$

where $D=D_{\text {hop }}+D_{\text {exc }}$ is the diffusion constant due to hopping $\left(D_{\text {hop }}\right)$ and exchange $\left(D_{\text {exc }}\right)$, and $T_{1}=\left(\omega_{\mathrm{HFI}}+\omega_{\mathrm{SOC}}\right)^{-1}$ is the spin relaxation (SR) time originated from hyperfine interaction (HFI) and spin-orbit coupling (SOC).

The hopping diffusion constant, $D_{h o p}=\frac{\mu k_{B} T}{e}$, is calculated based on the mobility $\mu=1 \frac{\mathrm{cm}^{2}}{V s}$ for PBTTT and $\mu=0.1 \frac{\mathrm{cm}^{2}}{V s}$ for P3HT taken from experiments at $300 \mathrm{~K}$ [179]. The exchange contribution to the spin diffusion constant for isotropic Heisenberg chain [164] is $D_{\text {exc }}=$ 1.6J $(R) R^{2}$, where $J(R)$ is the exchange interaction and $R$ is the average distance between the spins.

We use the expression for $J(R)=0.821\left(e^{2} / \varepsilon \xi\right)(R / \xi)^{5 / 2} e^{-2 R / \xi}$, given in Ref [125], where $e$ and $\varepsilon=2$ are the fundamental charge and dielectric constant, respectively, and the polaron localization length, $\xi=0.4 \mathrm{~nm}$, calculated from our first principles calculations.

As an electron hops between neighbouring molecules, it experiences a local HF field pointing in a random direction, which causes spin relaxation (SR). The SR rate due to the local HFI [125], [126] is $\omega_{H F I}=2 \Omega_{H F I}^{2} \tau / 3$ where $\Omega_{H F I}=2 \times 10^{8} \mathrm{~Hz}$ is the Larmor frequency of the local HF field (the hyperfine fields for PBTTT and P3HT are approximately 10 gauss, see 
Appendix A.III), the time $\tau=\left(2 D_{\text {hop }} / a^{2}+2 D_{\text {exc }} / R^{2}\right)^{-1}$, is a measure of the dwell time of a spin on a molecule in $1 \mathrm{D}$ and $a=0.45 \mathrm{~nm}$ is the average hopping distance between polarons along the $\pi-\pi$ stacking direction.

The SR rate due to the SOC is $\omega_{S O C}=2 \chi^{2} \tau^{-1}$, where $\chi^{2}=(4 / 3) \gamma^{2}$ is the spin-mixing parameter due to the SOC. We calculate $\gamma^{2}$ from electronic structure calculations from first principles theory (Appendix A.II).

According to Fig 6.8a in the main text, at high carrier concentrations, the exchange-induced diffusion constant is roughly two orders of magnitude higher than hopping diffusion constant. At this concentration, the spins move much faster and therefore the dwell time decreases. A decrease in the dwell time reduces the SR rate due to HFI. Our calculations show that replacing the HF field with a value which is two orders of magnitude larger, would only decrease the SDL by $2 \%$. On the other hand, as spins move faster, the spin flip rate due to SOC increases, which makes the SOC the dominant SR mechanism. However, a very small $\gamma^{2}$ for PBTTT and P3HT, increases the SR time caused by SOC and allows for a long SDL.

\section{Calculation of $\gamma^{2}$ and polaron-localization length}

The up-down spin-admixture parameter $\gamma^{2}$ describes the mixing of opposite spin states due to SOC and determines the rate of spin-flipping at charge hops in a semiconductor [128], [127]. Consequentially, an accurate determination of $\gamma^{2}$ is crucially important for theoretical predictions of SR rates in OSCs. $\gamma^{2}$ is the first order perturbation theory correction to the spinorbit free Hamiltonian.

We estimate $\gamma^{2}$ using the approach suggested by Yu [128], but with two main improvements. While $\gamma^{2}$ was originally derived within the restricted open-shell Hartree-Fock (ROHF) approximation to the density-functional theory (DFT) wave-function, we generalize its formulation to the unrestricted HF (UHF) approximation. This significant reduction in variational constraints on the wave-function used to obtain $\gamma^{2}$ correspondingly raises its predictive quality.

We further improve the quality of $\gamma^{2}$ by calculating SOC matrix elements from firstprinciples, within the Zeroth Order Regular Approximation (ZORA) [180] as implemented in the NWChem computational chemistry package [181]. The original formulation of $\gamma^{2}$ uses 
SOC constants (SOC matrix element prefactors) from experimental literature, i.e. observable 'orbitals'. Since this set of elements is only complete for the same number of orbitals in the theoretical treatment, the DFT calculations are restricted to very poor, minimal basis sets. Using SOC matrix elements from first-principles theory allows us to use basis sets of any quality. The single-zeta, polarized SARC basis set (ma-zora-def2-svp), which has been recontracted for ZORA, was found sufficient for the properties studied here. In order to remove linear dependencies carbon diffuse (minimal augmentation) functions were removed from the basis set, which was otherwise unmodified.

Finally, we choose a hybrid exchange-correlation functional specifically designed to improve the description of magnetic properties, PBE0 [182]. We approximate the charge to be delocalized over 4 monomeric units of PBTTT consistent with recent ESR characterisation [183].

Our estimates of $\gamma^{2}$ are summarized in Table A.1.

Table A. $1 \gamma^{2}$ calculated from DFT for PBTTT and P3HT.

\begin{tabular}{|l|l|}
\hline Material & $\gamma^{2}$ \\
\hline PBTTT & $3.154 \times 10^{-7}$ \\
\hline P3HT (dihedral $\left.\sim 20-30^{\circ}\right)$ & $1.268 \times 10^{-6}$ \\
\hline P3HT (dihedral $\left.\sim 40-60^{\circ}\right)$ & $2.416 \times 10^{-6}$ \\
\hline
\end{tabular}

The polaron localization length along the $\pi-\pi$ stacking direction, $\xi$, was determined using constrained-DFT calculations in NWChem using a def2-svp basis set and PBE0 [182] functional. Our system consists of three PBTTT chains in a $\pi-\pi$ stacking arrangement with a separation of $3.8 \AA$ with a charge constrained on the middle chain. However, as we're only interested in the $\pi$-electron density along the polymer backbone, we omit the alkyl chains to make our calculations faster. The spin-density is then averaged and plotted as a function of distance along the stacking direction in Fig A.1. From the figure, it is apparent that the polaron extent $(\xi)$ on the middle backbone (centered at 0 ) is about $4 \AA$ in the direction of transport. 


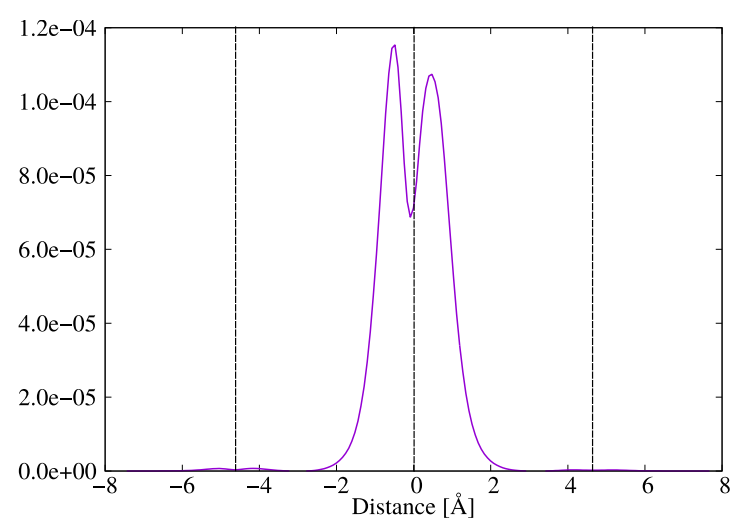

Figure A.1 Polaron (hole) density along $\pi$-stacking direction.

\section{Calculation of hyperfine fields}

Local hyperfine couplings in PBTTT and P3HT were calculated using the DFT package ORCA [184]. We used the PBE0 [182] exchange-correlation functional and IGLO-II basis set for all our calculations. The hyperfine fields are estimated using the following approximation from $\operatorname{Ref}[126]$ :

$$
B_{H}=\frac{2 \pi}{\hbar \gamma}\left[\sum_{\alpha}\left(A_{\alpha}^{2}+\frac{1}{3} \boldsymbol{T}^{\boldsymbol{\alpha}}: \boldsymbol{T}^{\boldsymbol{\alpha}}\right) I_{\alpha}\left(I_{\alpha}+1\right)\right]
$$

where $\gamma$ is the gyromagnetic coupling ratio, $\alpha$ is the atom index, $\mathrm{A}_{\alpha}$ is the isotropic Fermi contact term, $\mathbf{T}^{\alpha}$ is the anisotropic contribution to the hyperfine tensor and $I_{\alpha}$ are the scalar nuclear spins of relevant isotopes.

Using this we estimate the hyperfine fields for PBTTT and P3HT to be 6.1 Gauss and 6.7 Gauss respectively.

\section{Effect of Spin Dipole Interactions on Diffusion Constants}

While the theoretical framework mentioned above [Appendix A.I] treats spin-spin interactions due to exchange, it completely neglects dipole-dipole interactions between spins [129]. At the same time, the latter have been shown to play an important role in organic spintronic devices [185]. Accordingly, it is necessary to estimate the contribution of spin-spin dipole interactions 
to the spin diffusion coefficient in PBTTT. In this brief report we provide such an estimate.

First spin diffusion theory was developed nearly 70 years ago by Bloembergen to explain his experiments on spin-lattice relaxation in various insulating salts [186]. This theory gives a simple expression for the spin-diffusion coefficient $\mathrm{D}_{\mathrm{s}}$ :

$$
D_{s}=W_{s f} \overline{a^{2}}
$$

where $W_{s f}$ is the spin flip-flop rate (which Bloembergen assumed to be due to the spin-spin dipole interaction) and $a$ is the average distance between spins. Using the well-known expression for $W_{s f}$ available in the literature [187], one readily obtains the following result for the spin diffusion coefficient:

$$
D_{s}=\eta \frac{\mu_{0} \gamma^{2} \hbar}{r_{0}} c^{3 / 2} \frac{a}{r_{0}}
$$

where $\mu_{0}$ is the vacuum permeability, $\gamma$ is the gyromagnetic ratio, $r_{0}$ is the lattice constant, $\mathrm{c}=\mathrm{n}_{\mathrm{s}} \mathrm{r}_{0}^{3}$ is the dimensionless concentration of spin carriers, and $\eta$ is a dimensionless factor of order unity, which is determined by the morphology. The average distance between spins a can be related to the spin concentration as follows:

Substituting $a=r_{o} c^{-1 / 3}$ into Eq. (A.4), one obtains the following result for Ds:

$$
D_{s}=\eta \frac{\mu_{0} \gamma^{2} \hbar}{r_{0}} c^{5 / 6}
$$

which predicts the scaling of $D_{s}$ with the concentration of spin carriers as $D_{s} \sim \mathrm{c}^{5 / 6}$. This scaling prediction is in excellent agreement with the simulation results for $D_{s}$ available in the literature [188], thereby confirming the general validity of the theoretical approach outlined above.

Taking a typical value of the concentration of spin carriers employed in PBTTT experiments and a value for $\mathrm{r}_{0}$ from PBTTT morphology and substituting these values into Eq. (A.5) yields a value of $D_{s}$ which is roughly 3 orders of magnitude smaller than the corresponding contribution to $D_{S}$ due to spin-spin exchange interaction (at the same value of c). Accordingly, the spin-spin dipole interactions can be safely neglected in the theoretical treatment of spin diffusion in PBTTT. 


\section{Appendix B}

\section{Spin precession simulations (Hanle Effect) and measured spin lifetimes in organic semiconductors}

\section{Introduction}

If one simplifies the experimental measurement and considers just the DC component of the spin polarisation injected into the conjugated polymer due to spin pumping, i.e., one neglects the effect of the AC microwave field and the associated AC spin polarisation, the expected angular dependence of the ISHE voltage and the suppression of non-equilibrium spin polarisation due to spin precession under a varying external magnetic field $B$, can intuitively be understood by decomposing the injected spin polarization into components parallel and perpendicular to $B$.

We assume here that after diffusion through the conjugated polymer it is the projection of the remaining DC-component of the spin polarisation onto the z-axis, which generates the ISHE voltage $V_{\mathrm{ISHE}}^{x}$ in the Pt wire oriented along the x-axis (Fig B.1). At the Py/OSC interface, the DC-component is injected parallel to the equilibrium magnetization of Py, Meq, with an angle $\phi$ relative to the z-axis. The latter generally does not equal the angle $\theta$ of the external field. The ensemble spin polarization will therefore initially be tilted away from $B$ at an angle $\theta-$ $\phi$, causing a precession at the Larmor frequency $\omega_{L}=\gamma_{e}|B|$ where $\gamma_{e}$ denotes the charge's gyromagnetic ratio. This is depicted in Fig B.1 for a simplified 1D geometry where the Py and Pt interfaces are parallel and there is a single, well-defined channel length $L$. 


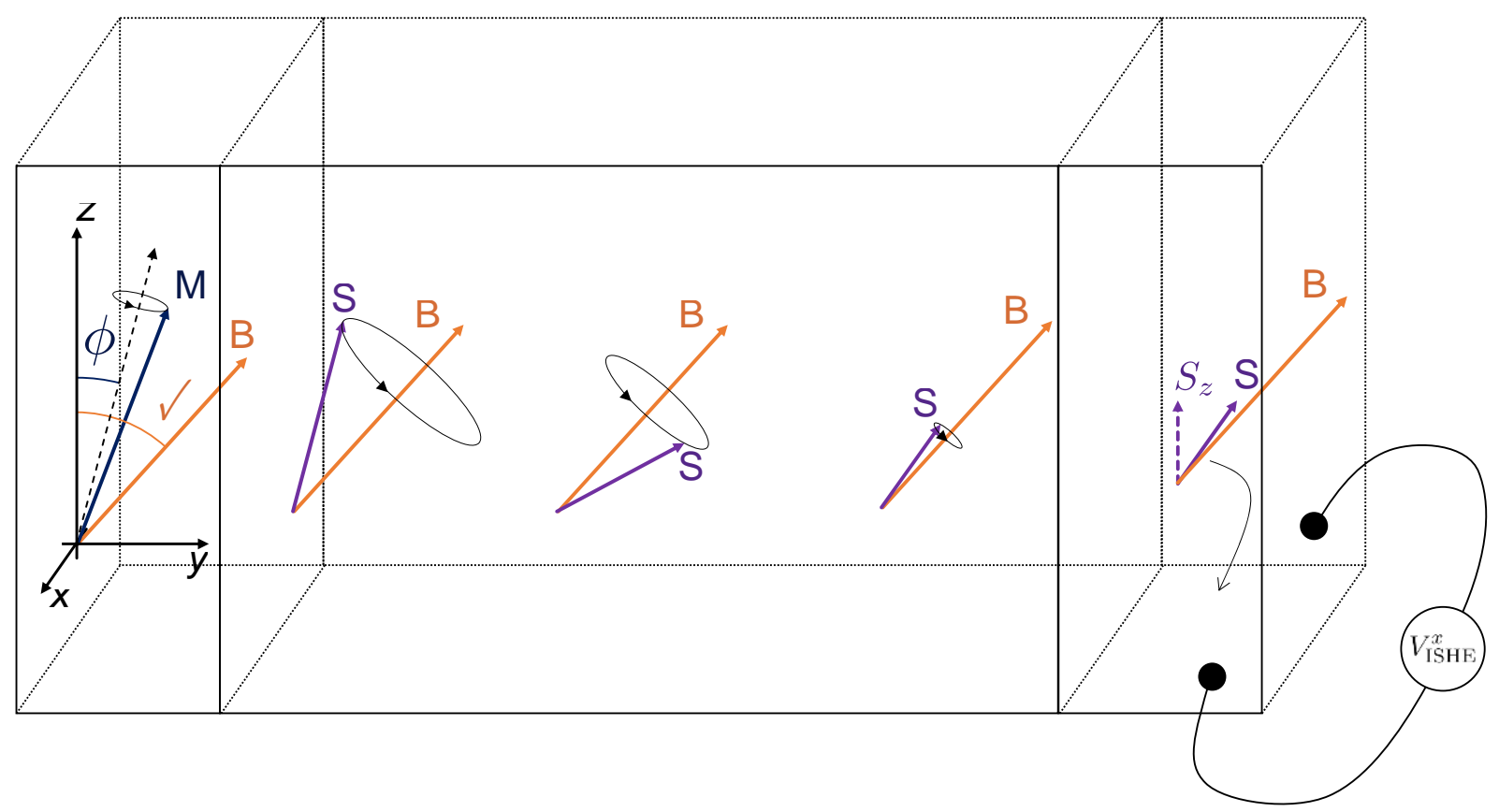

Figure B.1 Spin pumping geometry. Schematic of a simplified 1D spin-pumping geometry demonstrating the Hanle effect.

Only the component $S z$ of the ensemble spin-polarization which gets injected into Pt produces a detectable voltage along the $\mathrm{x}$-axis and $V_{\mathrm{ISHE}}^{\mathrm{x}}$ will therefore depend on the precession angle of $S$ at the Pt interface.

There are three effects acting on the spin-components $S^{\|}$and $S^{\perp}$ parallel and perpendicular to $B$ respectively, that determine the magnitude of $S z$ at the Pt interface: (1) Spin-lattice relaxation with a characteristic time $T 1$ will cause a decay of $S^{\|}$, (2) homogeneous and inhomogeneous dephasing will result in the decay of $S^{\perp}$ within a time $T 2 \leq T 1$, and (3) the distribution of diffusion pathways and different injection times will cause a distribution of phases that on average will reduce $S^{\perp}$.

This results in the Hanle effect, which has been treated in literature initially by Johnson and Silsbee for non-local spin valves, both phenomenologically by tracking and summing over the time evolution of all injected spins [189], and analytically by solving the resulting Blochdiffusion equation for the ensemble spin in the steady state [190]. The resulting expressions for the detectable spin signal have been widely and successfully applied to analyse the Hanle effect in non-local inorganic spin valves [191]. 


\section{A phenomenological approach}

Here, we will adapt the phenomenological approach from [189] to our FMR spin-pumping architecture which introduces a few key differences compared to literature: (1) The external field is no longer perpendicular to $S$ and its magnitude is determined by the FMR resonance condition. (2) Organic semiconductors often exhibit different spin lifetimes $T 1 \neq T 2$ which can result in $S^{\perp}$ decaying faster than $S^{\|}$. We accordingly treat parallel and perpendicular components of the injected spin $S 0$ separately with the decompositions shown in Fig B.2.

a

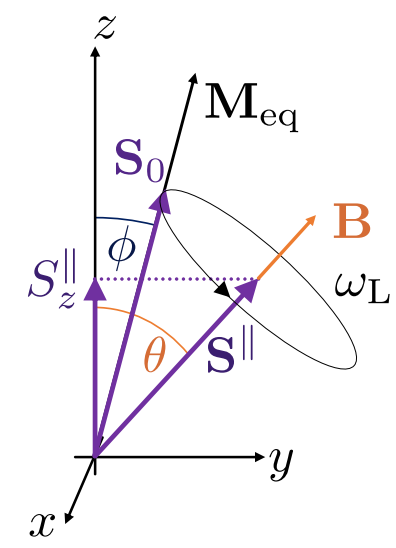

$S_{z}^{\|}=\cos (\theta) \cos (\theta-\phi)\left|\mathbf{S}_{0}\right|$ b

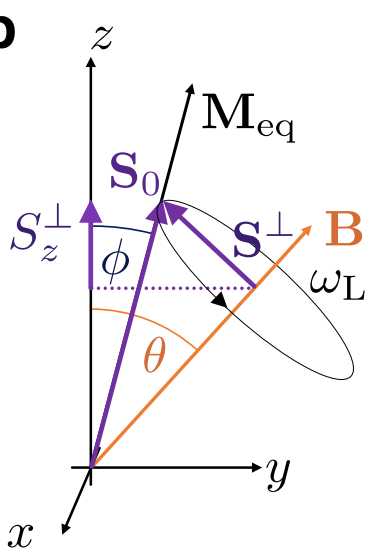

$S_{z}^{\perp}=\cos \left(\omega_{\mathrm{L}} t\right) \sin (\theta) \sin (\theta-\phi)\left|\mathbf{S}_{0}\right|$

Figure B.2 Schematic illustration of injected spin polarization. a, Decomposition of the injected spin polarization into components parallel and $\mathbf{b}$, perpendicular to the external field which exhibit different spin lifetimes.

The remaining spin-polarization for a spin injected at time $t=0$ after a diffusion length $L$ is given by the product of the distribution of arrival times for three-dimensional 3D diffusion

$$
P(t)=\frac{1}{\left(4 \pi D_{s} t\right)^{3 / 2}} e^{-\frac{L^{2}}{4 D_{s} t}}
$$

an exponential decay term $e^{-t / T_{1,2}}$, a precession term $\cos \left(\omega_{L} t\right)$, and the initial spin polarization. $D_{s}$ denotes the spin diffusion constant.

With the components $S_{Z}^{\|}$and $S_{Z}^{\perp}$ as given in Fig B.2, one obtains for the respective contributions to the ISHE voltage after integrating over all times: 


$$
\begin{gathered}
\mathrm{V}_{\mathrm{ISHE}}^{\| I} \propto \cos (\theta) \cos (\theta-\phi) \int_{0}^{\infty} \mathrm{P}(\mathrm{t}) \mathrm{e}^{-\frac{\mathrm{t}}{\mathrm{T}_{1}}} \mathrm{dt} \\
\propto \cos (\theta) \cos (\theta-\phi) e^{-\frac{L}{\sqrt{D_{s} T_{1}}}} \\
\mathrm{~V}_{\mathrm{ISHE}}^{\perp} \propto \sin (\theta) \sin (\theta-\phi) \int_{0}^{\infty} \mathrm{P}(\mathrm{t}) \cos \left(\omega_{\mathrm{L}} \mathrm{t}\right) \mathrm{e}^{-\frac{\mathrm{t}}{\mathrm{T}_{1}}} \mathrm{dt} \\
\propto \sin (\theta) \sin (\theta-\phi) \operatorname{Re}\left[\mathrm{e}^{\left.-\sqrt{\left(\frac{\mathrm{L}}{\sqrt{\bar{D}_{\mathrm{s}} \mathrm{T}_{2}}}\right)^{2}+\mathrm{i} \frac{\mathrm{L}^{2}}{\overline{\mathrm{D}_{\mathrm{s}}}} \omega_{\mathrm{L}}}\right]}\right.
\end{gathered}
$$

where the integrals in Eqs. (B.2) and (B.3) have been evaluated with Mathematica.

The resulting expressions are intuitive: The parallel spin polarization decays exponentially with the spin diffusion length

$$
\lambda_{\mathrm{s}}:=\sqrt{\mathrm{D}_{\mathrm{s}} \mathrm{T}_{1}}
$$

The perpendicular polarization on the other hand decays with the 'dephasing length' $\lambda_{2}=$ $\sqrt{D_{S} T_{2}}$ and for small $\omega_{L} L^{2} / D s \ll 1$ oscillates with the number of Larmor precessions during the mean transit time $t_{L}=L^{2} / D s$. When either the Larmor frequency or the channel length are increased, this oscillation becomes a decay since the distribution of arrival times causes an averaging over different angles in the precession. The contribution from $S_{z}^{\|}$is therefore destroyed both by dephasing and averaging over diffusion pathways.

We note that the same result for FMR spin-pumping has been derived by Ando and Saitoh by analytically solving a 3D Bloch-diffusion equation for the spin current in the steady state [59].

The total ISHE voltage then is the sum over longitudinal and transverse components 
$\mathrm{V}_{\mathrm{ISHE}}^{\mathrm{X}} \propto \mathrm{j}_{0} \cos (\theta) \cos (\theta-\phi) \mathrm{e}^{-\frac{\mathrm{L}}{\lambda_{\mathrm{S}}}}+\mathrm{j}_{0} \sin (\theta) \sin (\theta-\phi) \operatorname{Re}\left[\mathrm{e}^{-\sqrt{\left(\frac{\mathrm{L}}{\lambda_{2}}\right)^{2}+\mathrm{i} \frac{\mathrm{L}^{2}}{\mathrm{D}_{\mathrm{S}}} \omega_{\mathrm{L}}}}\right]$

where the absolute value of the injected spin current at the Py/OSC interface $j_{0}$ depends on the FMR resonance conditions and the spin mixing conductance [85]. It therefore varies when sweeping $\theta$ as shown in Fig B.3.
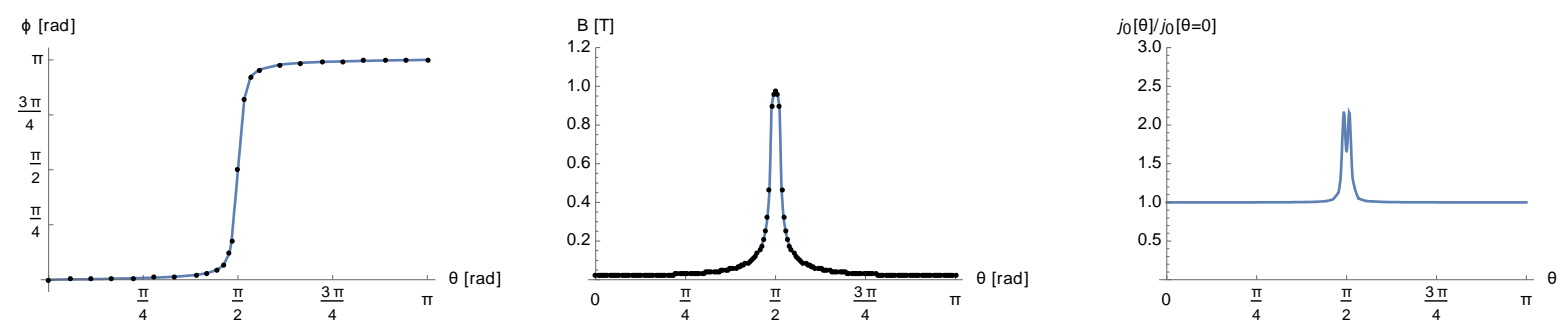

Figure B.3 Magnetisation dynamics. a, Angular dependence of the magnetization angle $\phi \mathbf{b}$, the FMR resonance field $B=|B| \mathbf{c}$, the injected spin current $j_{0}$ at the Py/OSC interface.

It is clear from Eq. (B.5) that a step-like profile resembling the experimental data is achieved when the in-phase contribution $V_{I S H E}^{\perp}$ remains significant. In the limit of rapid spin diffusion across the channel and a long dephasing time $T 2 \sim T 1$ a $\cos (\phi)$ angular dependence would be expected. Otherwise, "Hanle precession" will manifest itself as a more gradual, $\cos (\theta) \cos (\theta-\phi)$ angular dependence of $V_{I S H E}$.

Using the experimentally determined magnetization angle $\phi(\theta)$ and the magnitude of the external field $B(\theta)$ from Fig B.3, one can predict the expected angular dependence of $V_{\text {ISHE }}^{\mathrm{x}}$. The resulting plots are shown in Fig B.4 for different channel lengths. We fix the spin diffusion length at the experimental value for PBTTT of $\lambda \mathrm{s} \simeq 1 \mu \mathrm{m}$ and use the diffusion constant $D s=$ $1 \mathrm{~cm}^{2} \mathrm{~s}^{-1}$ for exchange-based spin diffusion. This implies a spin lattice relaxation time of $T 1=$ $10 \mathrm{~ns}$. From the negligible angular dependence of $\lambda \mathrm{s}$, we conclude that $T 1$ remains constant over the experimental range of magnetic fields between $100 \mathrm{mT}-1200 \mathrm{mT}$. Furthermore, consistent with the step-like angular dependence of $V_{\text {ISHE }}$ and Elliott-Yafet-like spin relaxation, we assume $T 2 \cong T 1$. 

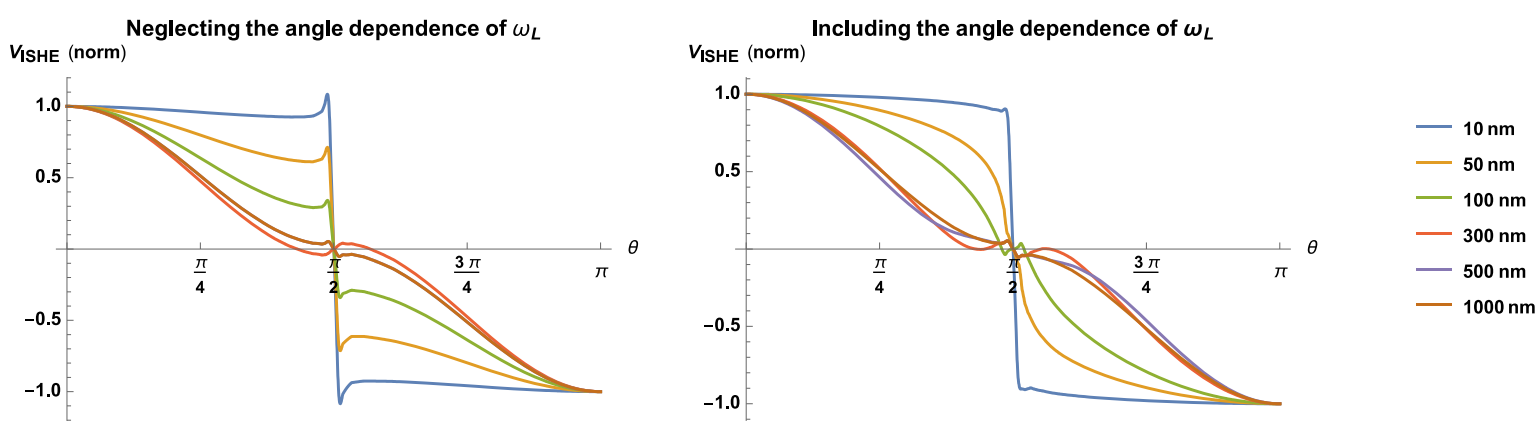

Figure B.4 LSP Hanle simulation. Predicted Hanle curves from a Bloch-diffusion model in the simplified geometry of Fig B.1 for experimentally determined parameters for channel lengths between $100 \mathrm{~nm}-1000 \mathrm{~nm}$.

It is evident from Fig B.4 that even for such rapid exchange-mediated spin diffusion, the model predicts a more gradual angular dependence than experimentally observed starting at $L=100$ $\mathrm{nm}$, even when neglecting the increase of the Larmor precession frequency for $\theta \rightarrow \pi / 2$. Assuming a shorter dephasing time $T 2<T 1$ further reduces the contribution of in-phase spins which lies at the origin of the step-like angular dependence.

Furthermore, the actual lateral experimental geometry with widths of $1 \mu \mathrm{m}$ and $400 \mu \mathrm{m}$ for the Pt detector and the Py injector, respectively, does not have a well-defined channel length. Instead, all possible 'channel lengths' within the spin-diffusion length can contribute to $V_{\text {ISHE}}$, resulting in an averaged signal with effective channel lengths up to $1000 \mathrm{~nm}$ even for a $\mathrm{Py} / \mathrm{Pt}$ separation of $100 \mathrm{~nm}$.

The model presented in this section suggests that although the exchange-enhanced spin diffusion is indeed significantly more rapid than charge-based spin diffusion the absence of a Hanle precession signature in the angular dependence of the ISHE voltage in our lateral spin pumping experiment cannot simply be explained by spin diffusion through the conjugated polymer occurring on a faster time scale than that of the spin precession around the applied magnetic field, as was suggested in Ref [125]. 


\section{Verification of ESR measured spin lifetimes for doped PBTTT}

We have attempted to measure independently the spin relaxation times $T_{1}$ and $T_{2}$ in our F4TCNQ doped PBTTT films using electron spin resonance spectroscopy (ESR). However, the overlapping ESR signals of F4TCNQ anions and doped PBTTT prevent an accurate estimation of spin relaxation times of doped PBTTT. To circumvent this problem, we have used an ion exchange method, in which the dopant radical anion is replaced by a closed shell anion that does not generate an ESR signature. The process involves first sequentially doping a PBTTT film with another strong oxidative, radialene-based dopant (CN6-CP) in an acetonitrile solution, followed by the exchange of the CN6-CP radical anions with bis(trifluoromethylsulfonyl)-imide (TFSI) anions using a concentrated lithium TFSI/DMSO solution. For the ESR measurements shown below, PBTTT:TFSI films were fabricated by exposing PBTTT:CN6-CP films to a $1 \mathrm{M}$ solution of Li TFSI (Sigma Aldrich) in dimethylsulfoxide for 60 seconds, then spinning at $5000 \mathrm{rpm}$. Fig B.5a shows the UV-vis-NIR spectra of films before and after ion exchange. The strong CN6 $-\mathrm{CP}^{\bullet-}$ absorption bands at 600 $\mathrm{nm}$ and $700 \mathrm{~nm}$ are no longer visible after exposure to the ion exchange solution, indicating that nearly all CN6 - $\mathrm{CP}^{\bullet-}$ ions have been replaced with TFSI [192]. After ion exchange, the films exhibit a similarly high conductivity as our F4TCNQ/PBTTT films $(\sigma=130 \mathrm{~S} / \mathrm{cm})$ and we therefore consider the TFSI-ion exchanged films to provide a representative measure of the spin relaxation times of doped PBTTT.
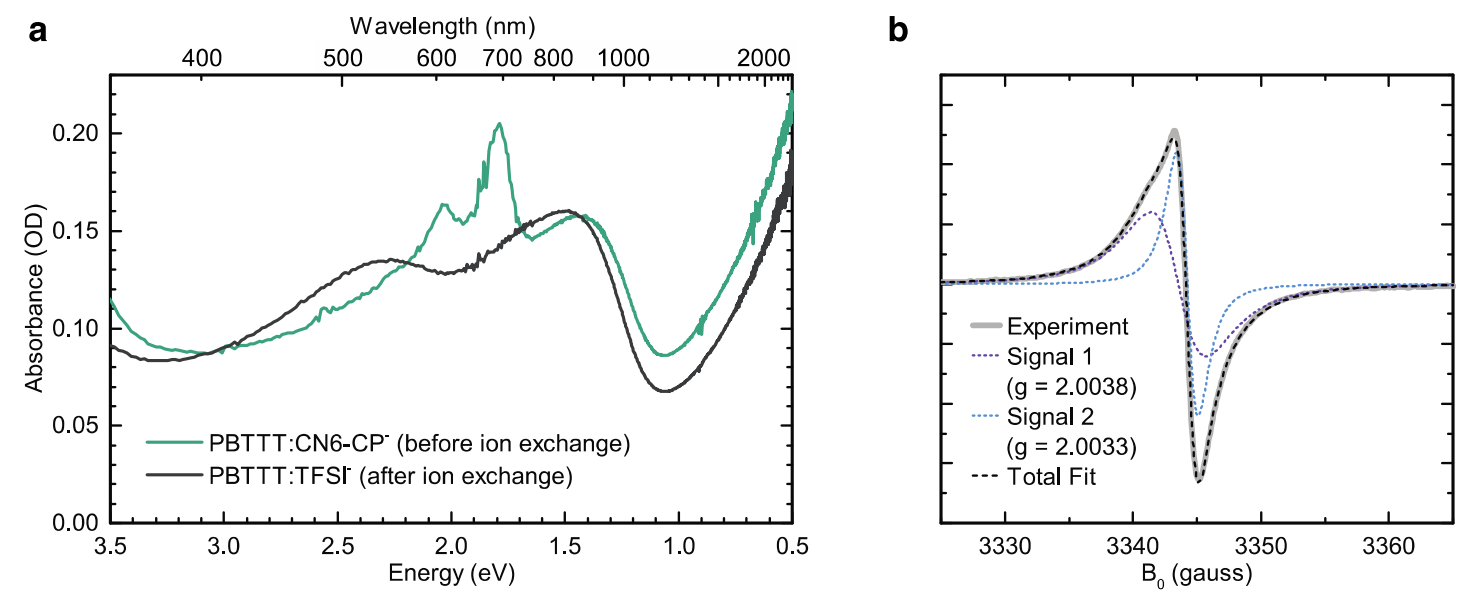

Figure B.5 Ion-exchanged PBTTT ESR and UV-vis spectrum. a, UV-vis-NIR spectra of a PBTTT:CN6-CP film before and after ion exchange with Li TFSI $\mathbf{b}, \mathrm{X}$-band ESR spectrum of a PBTTT:TFSI film, along with fit obtained from a simultaneous fit to power saturation data. Spectrum 
shown was taken at $20 \mathrm{~mW}$ microwave power.

Because TFSI is a closed shell anion, the ESR spectrum of PBTTT:TFSI should originate purely from the PBTTT polarons, permitting us a clear measurement of their spin relaxation times. Fig B.5b shows a representative spectrum taken from a power saturation experiment. The ESR signal shows significant asymmetry, which can be well fit at all microwave powers by two overlapping signals. The broader of these signals at $g=2.0038$ contains the majority of the spin density population $\left(\mathrm{N}_{\text {spin }}=1.02 \pm 0.08 \times 10^{20} \mathrm{~cm}^{-3}\right)$ and shows shorter relaxation times $\left(\mathrm{T}_{1}=22.9 \pm 3.7 \mathrm{~ns} ; 15.37 \pm 0.08 \mathrm{~ns}\right)$. These values nicely match with those obtained from spin diffusion length measurements. The second signal at $\mathrm{g}=2.0033$ corresponds to a lower spin density $\left(2.64 \pm 0.22 \times 10^{19} \mathrm{~cm}^{-3}\right)$ and shows considerably longer relaxation times $\left(\mathrm{T}_{1}=209.3\right.$ $\pm 4.0 \mathrm{~ns} ; 45.0 \pm 1.0 \mathrm{~ns}$ ). This second signal is too strong to originate from residual CN6 $-\mathrm{CP}^{\bullet-}$ or impurities, and therefore is tentatively assigned to a second polaron species, that is potentially more localised. 


\section{Appendix C}

\section{Lithography processes details}

\section{E-beam lithography}

Undiluted PMMA A4 solution was spin coated on the substrate at $6000 \mathrm{rpm}$ for $30 \mathrm{~s}$ to deposit around $300 \mathrm{~nm}$ PMMA resist layer on the substrate. The resist was then baked at $180^{\circ} \mathrm{C}$ for 5 minutes. The e-beam lithography writing was achieved by exposing the resist to electron beam with the following parameters: beam current $=100 \mathrm{pA}-5 \mathrm{nA}$ depending on the feature size resolution, dose time $=1 \mu \mathrm{s}$. The device pattern was designed using CAD software. The patterned resist was developed by immersing the sample in a developer solution consists of one part of methyl isobutyl ketone to three parts of isoproponal for $45 \mathrm{~s}$. Following the metal layer deposition, the lift-off was performed by immersing the sample in acetone which dissolves the PMMA resist.

\section{Patterned OSC Hall bar fabrication}

The bottom contact electrodes for the Hall bar structure was fabricated by optical lithography patterning, followed by $\mathrm{Cr}(5 \mathrm{~nm}) / \mathrm{Au}(25 \mathrm{~nm})$ metal deposition and finally lift-off. Organic semiconductor such as PBTTT was then spin coated on top of the electrodes. CYTOP was spin coated on top of PBTTT from CYTOP:Solvent solution (1:2) at $2000 \mathrm{rpm}$ for $20 \mathrm{~s}$ to protect PBTTT from subsequent processing. CYTOP layer was annealed at $80^{\circ} \mathrm{C}$ for 20 minutes. 1.5 $\mathrm{nm}$ thick Al was evaporated using thermal evaporation on top of the structure at a rate of 0.5 $\AA / \mathrm{s}$. The Al film was then exposed to atmosphere to oxidise Al. A layer of Photoresist, S1813 was spin coated on top of the structure at $6000 \mathrm{rpm}$ and $30 \mathrm{~s}$. The resist was baked at $120^{\circ} \mathrm{C}$ for 2 minutes. The resist was then patterned by optical lithography to establish the Hall bar pattern. The resist was then developed in developer, MF319 for 30 s. Finally, the structure was rinsed with deionised water. 
The device was then placed in an oxygen plasma asher under $300 \mathrm{~W}$ applied power for 15 minutes to etch away the organic semiconductor that is not protected by the photoresist. A scotch tape was used to remove photoresist and CYTOP on top of the organic semiconductor. This process works well since the adhesion between CYTOP and organic semiconductor is weak.

\section{Orthogonal resist patterning}

After the deposition of organic semiconductor such as PBTTT, orthogonal resist, OsCoR 4000 was spin coated at $2000 \mathrm{rpm}$ for $30 \mathrm{~s}$. The resist was vacuum annealed by placing the sample in a vacuum chamber with pressure below $10^{-2}$ mbar for 30 minutes. Optical resist, S1813 was then spin coated on top of the orthogonal resist at $5000 \mathrm{rpm}$ for $30 \mathrm{~s}$. The optical resist was again vacuum annealed for 30 minutes as done previously for the orthogonal resist. The optical resist was exposed to UV light through a lithography mask with desired pattern for $12 \mathrm{~s}$. The resist was then developed in MF319 for $40 \mathrm{~s}$, followed by $40 \mathrm{~s}$ immersion in deionised water. The exposed orthogonal resist beneath the developed optical resist was dissolved away by gently agitating the sample in HFE solution for $90 \mathrm{~s}$. The sample was then blow dried by $\mathrm{N}_{2}$ gas. Following the metal layer deposition, the lift-off was performed by immersing the sample in HFE for at least 2 hours which dissolves the orthogonal resist. 


\section{Appendix D}

\section{Publications and Presentations}

\section{Publications}

Long range lateral spin transport in conjugated organic polymers measured at room temperature

Shu-Jen Wang, Deepak Venkateshvaran, M. R. Mahani, Uday Chopra, Erik R. McNellis, Riccardo Di Pietro, Angela Wittmann, Guillaume Schweicher, Keehoon Kang, Murat Cubukcu, Remington Carey, Sebastian Müller, Sergei A. Egorov, Janis N. M. Siebrecht, Thomas J. Wagner, Cameron Jellett, Mark Little, Iain McCulloch, Joerg Wunderlich, Jairo Sinova, and Henning Sirringhaus,

to be submitted (2018).

Thermo-electrical detection of topological protected magnetic textures on the track M. Cubukcu, D. Venkateshvaran, A. Wittmann, S-J. Wang, P.E. Roy, S. Auffret, L. Vila, H. Sirringhaus, and J. Wunderlich,

Applied Physics Letters (2018).

\section{Presentations}

Spin transport in chemically doped conjugated organic polymers (Oral talk)

S.-J. Wang, D. Venkateshvaran, R. Mahani, E. R. McNellis, R. Di Pietro, G. Schweicher, M. Cubukcu, K. Kang, A. Wittmann, T. J. Wagner, C. Jellett, J. N. M. Siebrecht, U. Chopra, M. Little, I. McCulloch, J. Wunderlich, J. Sinova, H. Sirringhaus, 9th Joint European Magnetic Symposia (JEMS) Conference, Mainz, Germany (2018). 
Long range lateral spin transport in conjugated organic polymers (Invited talk)

Shu-Jen Wang, Deepak Venkateshvaran, Erik McNellis, Uday Chopra, Reza Mahani, Angela Wittmann, Riccardo Di Pietro, Guillaume Schweicher, Keehoon Kang, Murat Cubukcu, Thomas J. Wagner, Cameron Jellet, Mark Little, Iain McCulloch, Joerg Wunderlich, Jairo Sinova, and Henning Sirringhaus,

7th International Meeting on Spin in Organic Semiconductors (SpinOS), Halle, Germany (2018)

Spin Current Transmission in Conjugated Organic Polymers (Oral talk)

Shu-Jen Wang, Deepak Venkateshvaran, Reza Mahani, Erik R. McNellis, Riccardo Di Pietro, Murat Cubukcu, Thomas J. Wagner, Angela Wittmann, Guillaume Schweicher, Keehoon Kang, Cameron Jellet, Mark Little, Iain McCulloch, Joerg Wunderlich, Jairo Sinova, and Henning Sirringhaus,

7th International Meeting on Spin in Organic Semiconductors (SpinOS), Halle, Germany (2018)

Electrically detected spin transport in conjugated organic polymers (Poster)

Shu-Jen Wang, Deepak Venkateshvaran, Riccardo Di Pietro, Murat Cubukcu, Thomas J. Wagner, Erik R. McNellis, Angela Wittmann, Guillaume Schweicher, Keehoon Kang, Cameron Jellet, Mark Little, Iain McCulloch, Joerg Wunderlich, Jairo Sinova, and Henning Sirringhaus,

21st International Conference on Magnetism (ICM), San Francisco, USA (2018)

Spin transport in organic semiconductors (Oral talk)

S-J. Wang, A. Wittmann, K. Kang, S. Schott, G. Schweicher, R. Di Pietro, J. Wunderlich, D. Venkateshvaran, M. Cubukcu and H. Sirringhaus, 4th International Symposium on Advanced Magnetic Materials and Applications (ISAMMA), Phy Quoc, Vietnam (2017)

Spin transport in organic semiconductors: From spin pumping by ferromagnetic resonance to lateral spin-valves (Poster)

S-J. Wang, A. Wittmann, K. Kang, S. Schott, G. Schweicher, R. Di Pietro, J. Wunderlich, D. Venkateshvaran, M. Cubukcu and H. Sirringhaus, IEEE International Magnetics Conference (INTERMAG), Dublin, Ireland (2017) 